\title{
Wavelengths, Intensities, and Zeeman Patterns in Ytterbium Spectra (Yb I, II, III, IV)
}

\author{
William F. Meggers and Charles H. Corliss
}

(October 19, 1965)

\begin{abstract}
In 1937, Meggers and Scribner published a paper on arc and spark spectra of ytterbium providing wavelengths, relative intensities, and spectrum numbers of 1668 spectral lines, including about 400 for $\mathrm{Yb}$ I, 1250 for $\mathrm{Yb}$ II, and 12 for $\mathrm{Yb}$ III. That work was handicapped by impure materials and conventional light sources. When pure ytterbium metal and new light sources became available in 1950, Meggers and Corliss decided to make a new description of ytterbium spectra. This new description includes data for 7300 spectral lines with wavelengths between $2000 \AA$ and $12000 \AA$ distributed as follows: 1800 belong to $\mathrm{Yb} \mathrm{I}, 5100$ to $\mathrm{Yb} \mathrm{II}, 430$ to $\mathrm{Yb}$ III, and 5 to $\mathrm{Yb}$ IV. The Zeeman effect on 1300 lines has been observed in magnetic field intensities ranging from 3.7 to 9.358 tesla (37000 to 93580 gauss). These data were obtained for chemical identifications and for structural analyses of the first two spectra of ytterbium.
\end{abstract}

Key Words: Spectra of ytterbium: ytterbium. spectra of: Zeeman effect in ytterbium; wavelengths of ytterbium.

\section{Introduction}

Although ytterbium was discovered in 1907, Meggers and Scribner [1] ${ }^{1}$ provided, in 1937, the first useful description of conventional are and spark spectra of this element. That publication reviewed all earlier work and presented measured wavelengths of 1668 spectral lines ranging from $2073.70 \AA$ to $10321.64 \AA$ and estimated relative intensities from 1 to 2000. On the basis of relative intensities in arc and spark light sources, that description included the first deliberate assignment of lines to three successive spectra (about 400 to $\mathrm{Yb} \mathrm{I}, 1250$ to $\mathrm{Yb} \mathrm{II}$, and a dozen to $\mathrm{Yb} \mathrm{III}$ ), and for the first two provided the identification of electron configurations, spectral terms and series.

During the first half of this century, the major obstacle in making useful descriptions of the spectra that characterize the so-called rare earths was the difficulty in obtaining pure samples. For example [1] "our first measurements of $\mathrm{Yb}$ spectra were made in 1929 when samples of $\mathrm{Lu}$ and $\mathrm{Yb}$ oxides, prepared by Auer and obtained from Eder in 1919, were available. Although the chemical separation was far from complete, it was possible to make a fairly satisfactory assignment of lines to $\mathrm{Lu}$ and $\mathrm{Yb}$ by simultaneous comparison of are and spark. However, both salts contained some Tm as impurity which could not be completely identified without comparable data for Tm spectra. The first sample of Tm salt available for this purpose was generously supplied in 1933 by B. S. Hopkins, University of Illinois, who also supplied three samples of $\mathrm{Yb}$ oxide; one of which contained more Lu than Tm, another more Tm than Lu, and the third was entirely free from both $\mathrm{Lu}$ and Tm but contained considerable lanthanum."

Through the courtesy of G. R. Harrison, Meggers

'Figures in brackets indicate the literature references on page 106. in 1939 photographed the first Zeeman patterns of ytterbium lines by using the Bitter magnet and the concave gratings at the Massachusetts Institute of Technology. These results were an accidental by-product of the first attempt to observe the magnetic splitting of thulium lines, as the purest sample of thulium then available contained about 15 percent of ytterbium. These Zeeman data, and further observations of spark spectra, enabled Meggers [2] to confirm and greatly extend the earlier quantum interpretation of the $\mathrm{Yb}$ II spectrum [1], but it also became obvious that the structural analyses of $\mathrm{Yb}$ spectra could not be satisfactorily extended without additional experimental data.

Since 1945, several contributions to the data of ytterbium spectra have been presented by others. Gatterer and Junkes [3] presented wavelengths and relative intensities of 1476 ytterbium lines observed in arc and spark spectra between $2262.25 \AA$ and $7448.33 \AA$, but most wavelengths were taken from earlier tables, estimated intensities were given on a scale of 1 to 10 , and in many cases it was impossible to sort the lines into $\mathrm{Yb}$ I and $\mathrm{Yb}$ II spectra.

A valuable extension to information concerning ytterbium spectra was made in 1959 by Humphreys and Paul [4] who used electrodeless lamps. They reported the wavelengths and relative intensities of 49 infrared lines dispersed by a grating spectrometer and detected with a lead-sulfide cell. The wavelengths ranged from $10267.05 \AA$ to $24,552.51 \AA$ and the intensities from 3 to 30000; no separation of $\mathrm{Yb}$ I and $\mathrm{Yb}$ II was attempted.

In 1961, Bryant [5] reported on "the spectra of doubly and triply ionized ytterbium." By using three different electrical discharges (hot spark, mild spark, high-voltage arc), Bryant succeeded in photographing 5900 lines (from $677 \AA$ to $11262 \AA$ ); he attempted to assign each line to its proper spectrum, either $\mathrm{Yb} \mathrm{I}$, $\mathrm{Yb} \mathrm{II}, \mathrm{Yb} \mathrm{III}$, or $\mathrm{Yb} \mathrm{IV}$. His list contains 4550 lines with wavelengths greater than $2000 \AA$. We have 
changed some of his assignments, failed to find some of his lines, but increased the total number longer than $2000 \AA$ by 60 percent.

In 1962, Allen [6] reported on "the Zeeman effect of the arc and spark spectra of ytterbium and thulium." Those spectra were excited in a d-c arc between impregnated electrodes mounted in bored poles of an electromagnet with a maximum field intensity of 2.55 tesla (25500 gauss). Although Zeeman patterns are tabulated for 350 ytterbium lines, only 20 are resolved; most of the remainder are listed as pseudo triplets or quartets.

Prior to 1947, individual (lanthanon) rare earths were usually extracted from natural mixtures by fractional crystallization. In order to obtain a comparatively pure sample, it was often necessary to repeat this operation thousands of times. A more efficient technique, ion-exchange chromatography involving the use of absorption columns of organic resins, was developed in 1947. By this method since 1950, large quantities of $(99.99 \%)$ pure lanthanons have been accumulated as by-products of the purification and concentration of thorium and uranium as nuclear fuels. Thus, the former major handicap to spectroscopic investigation of lanthanon spectra has vanished and high-purity metals are now obtainable from various commercial sources at moderate cost [7]. Furthermore, since 1950, new types of light sources such as electrodeless lamps excited by microwaves, and sliding sparks, have been developed. These emit stronger and cleaner spectra than the d-c arcs and a-c sparks previously used at atmospheric pressure. These recent important improvements in materials and methods of observing persuaded us, in 1958, to undertake another description of ytterbium spectra. The present description contains 7300 lines (2000 to 12000 A) and 1300 Zeeman patterns; it should be adequate for chemical identifications and for further progress in the structural analyses of ytterbium spectra. In fact, new analyses and quantum interpretations of $\mathrm{Yb} \mathrm{I}$ and $\mathrm{Yb}$ II are now approaching completion and will be reported in separate papers.

\section{Experiments}

To obtain spectra of rare-earth elements, it was formerly customary to burn chemical compounds (oxides) of them on carbon, copper, or silver electrodes of electrical arcs or sparks at atmospheric pressure. Those sources favored the excitation of ionic spectra, masked many lines near those of the electrode materials, or of atmospheric gases, and caused undue widening of the lines because of high temperature and pressure. During the past decade, all these defects have been removed by the introduction of electrodeless evacuated lamps containing minute samples of metallic halides excited at moderate temperatures and reduced pressures by microwaves, as described by Corliss, Bozman, and Westfall [8] and demonstrated by Corliss and Meggers [9] for hafnium.

Our first ytterbium lamps were made with bromine compounds but the $\mathrm{Yb}$ spectra were found to contain, in addition to $\mathrm{Br}$ lines, many troublesome diatomic molecular bands due to $\mathrm{YbBr}$. Since $\mathrm{Yb}$ has relatively low melting $\left(824{ }^{\circ} \mathrm{C}\right)$ and boiling $\left(1193^{\circ} \mathrm{C}\right)$ points, new lamps were made containing only pure metal and a small pressure of noble gas as described by Meggers and Westfall [10] and by Zelikoff, Wyckoff, Aschenbrand, and Loomis [11]. Excited with microwaves, these lamps produced extremely intense $\mathrm{Yb}$ I spectra and most of $\mathrm{Yb}$ II but only a trace of $\mathrm{Yb}$ III. A slightly different form of lamp, also containing pure $\mathrm{Yb}$ metal and noble gas, was operated as a ring-discharge with high frequency in a surrounding solenoid; it greatly enhanced the intensity of $\mathrm{Yb}$ II relative to $\mathrm{Yb}$ I, and in addition excited Yb III and Yb IV peripherally.

In 1937, Meggers and Scribner [1] observed only 400 lines belonging to neutral ytterbium atoms and remarked that "The $\mathrm{Yb}$ I spectrum is surprisingly simple but may not be fully developed in the arc." In 1958, when Corliss and Meggers [9] undertook to make an improved description of hafnium spectra, they found that the number of recorded lines belonging to $\mathrm{Hf}$ I was greatly increased by using (instead of $d$-c arcs) electrodeless lamps containing hafnium halides excited by microwaves. This experience prompted us to apply this type of excitation to ytterbium halides to obtain a more complete development of the first spectrum. Our first ytterbium lamps, accordingly, were charged with $\mathrm{YbBr}_{3}$ prepared by dissolving pure $\mathrm{Yb}_{2} \mathrm{O}_{3}$ in a mixture of $\mathrm{HBr}$ and $\mathrm{Br}_{2}$ in water solution at about $300{ }^{\circ} \mathrm{C}$. The spectra emitted by these lamps were satisfactory in the ultraviolet, but the visible region was partly obscured by strong bands of $\mathrm{YbBr}$. We then decided to avoid the use of metal halides since ytterbium has relatively low melting and boiling points. A 100-mg portion of pure $\mathrm{Yb}$ metal was placed in a 7-mm o.d. quartz tube with a hemispherical window blown at one end. This tube was evacuated, outgassed, and filled with 7 torr of argon gas. With the argon excited in a microwave field of $2450 \mathrm{MHz}$, a Bunsen flame was applied to the portion of the tube containing the ytterbium metal. A green glow, characteristic of the arc spectrum of ytterbium, appeared and became dazzlingly brilliant as the temperature rose to make the quartz tube a dull red. At that temperature $\left(\right.$ ca. $600{ }^{\circ} \mathrm{C}$ ), ytterbium has a vapor pressure of about 0.01 torr. The lifetime of this type of lamp is limited by the diffusion and ultimate condensation of ytterbium vapor onto the window, which then becomes opaque. In our lamps, this generally took place after about $20 \mathrm{hr}$ of operation. Spectral regions where argon lines interfere with ytterbium were reobserved with lamps containing pure $\mathrm{Yb}$ metal and helium gas. In that case, a reservoir with a capacity of about 50 to $100 \mathrm{~cm}^{3}$ was necessary to maintain the gas pressure because helium gradually escapes through hot quartz.

These ytterbium lamps emit an intense and very clean spectrum consisting primarily of $\mathrm{Yb}$ I with numerous lines of $\mathrm{Yb}$ II present but none of $\mathrm{Yb}$ III. Because $\mathrm{YbI}$ and $\mathrm{Yb}$ II contain some prominently outstanding lines, our first spectrograms on nonbacked photographic plates were marred by opaque patches 
of halation extending $1 \mathrm{~cm}$ on either side of the strong images; all subsequent exposures were made on black-backed plates.

To distinguish and identify successive spectra of ytterbium, a light source of higher excitation was required for comparison. This we found in the ringdischarge discovered, named, and described by J. J. Thompson [12] in 1891. Although Thompson suggested that his light source might serve spectroscopy, it never became popular. However, in 1923, the Bloch brothers [13] demonstrated that the proper use of a Thompson tube permitted them to record simultaneously four successive spectra of mercury and distinguish them by observing different intensity gradients along the diameter or radius of the tube. Nearly 40 years later, in like manner, we succeeded in recording simultaneously four successive spectra of ytterbium, distinguished by radial intensity gradients in spectral-line images obtained from a single source, the ring-discharge or Thompson tube.

In our experiments, a $100 \mathrm{mg}$ lump of pure ytterbium metal was placed in a Vycor tube $(18 \mathrm{~mm}$ o.d. and $150 \mathrm{~mm}$ long) closed at one end with a plane quartz window. This tube was evacuated, outgassed, and sealed after being filled with helium to a pressure of 6 torr. The tube was then surrounded by a solenoid ( $25 \mathrm{~mm}$ diam by $140 \mathrm{~mm}$ long) of 20 turns of platinum wire which was connected into the discharge circuit of a high-voltage spark apparatus with capacitance of $0.001 \mu \mathrm{F}$ charged to $20,000 \mathrm{~V}$ and discharged through a 6-mm gap. Peak current in the solenoid was about 500 A.

The platinum solenoid and the enclosed ytterbium lamp were mounted with their axes on the optical axis of the spectrograph, and the tube was focused on the slit. When excited, the cold tube emitted a dull reddish glow due to the helium present. A Bunsen burner set beneath the solenoid brought the platinum coils to a bright red heat and the discharge in the tube changed to a bright blue-green as ytterbium was vaporized and excited.

Unlike the ordinary spark in air, the Thompson tube produces clean ytterbium spectra of sharp lines. When it is photographed beside the microwave discharge, the spectra of neutral and ionized atoms are easily distinguished. The first spectrum is strongest in the microwave discharge and considerably weaker in the ring discharge where it appears with maximum intensity on the axis of the tube. The second spectrum is generally much stronger and more fully developed in the ring discharge than in the microwave discharge because the latter lacks energy for the excitation of higher states in ions. The third and fourth spectra appear only in the ring discharge where they are observed only near or at the walls of the tube, fading toward the axis.

It appears that the energy for excitation of spectra in the Thompson tube has its minimum value on the axis and maximum at the walls. Consequently, when the tube is viewed end-on, by focusing the diameter of the tube on the slit of a stigmatic spectrograph, the lines characteristic of neutral atoms are stronger at the center and lines belonging to singly ionized atoms are nearly uniform in intensity along the diameter, whereas lines due to doubly or trebly ionized atoms are absent at the center but are observed near the wall with noticeably different intensity gradients.

The spectra of microwave and ring discharges of ytterbium were photographed side-by-side together with short images of superposed standards from iron arcs or from thorium-iodide tubes. To disperse and focus the spectra, four concave gratings of $6.5 \mathrm{~m}$ radius, in Wadsworth-type mountings, were used. The short ultraviolet region from $2000 \AA$ to $2500 \AA$ was photographed in the first order of two (30,000 lines per inch) gratings with a plate factor of $2.4 \AA / \mathrm{mm}$. In the range $2500 \AA$ to $4500 \AA$, the spectra were recorded in the second order of either grating with a plate factor of $0.87 \AA / \mathrm{mm}$, and the same setting served to record the range $5000 \AA$ to $9000 \AA$ in the first order with a plate factor of $1.74 \AA / \mathrm{mm}$. In the latter case, a colored glass filter was placed before the slit to absorb the second and third orders, and special dye-sensitized plates recorded the first order. The spectral range $7000 \AA$ to $10000 \AA$ was photographed with a third grating having 15,000 lines per inch and plate factor of $5 \mathrm{~A} / \mathrm{mm}$, and the infrared observations were extended to $12000 \AA$ with a fourth grating, ruled 7,500 lines per inch, whose plate factor was $10 \AA / \mathrm{mm}$.

The above-mentioned spectrograms yielded wavelengths and estimated relative intensities of about 7000 ytterbium lines and, with few exceptions, they all appeared as sharp emission lines. In particular, there was no detectable absorption or self-reversal in any lines emitted by our sources. After our line list was compiled, Sugar [14] developed a new light source specifically for producing self-reversed lines in both the first and second spectra of rare-earth elements. The first observations with this light source were made in 1962 by Sugar with ytterbium electrodes. Those spectrograms (covering $2400 \AA$ to $7000 \AA$ ) were evaluated by Meggers who found that 22 lines of $\mathrm{Yb}_{\mathrm{I}}$ and 50 of $\mathrm{Yb}$ II were absorbed or self-reversed; these are indicated by $\mathrm{A}$ and by $\mathrm{R}$ or $r$ respectively, following the intensity number in our table of ytterbium spectra.

The final contribution to our table of ytterbium lines was made in 1963 by Nissan Spector who was photographing the spectra of erbium emitted by a sliding spark; he kindly substituted ytterbium electrodes for erbium and photographed the spectral range $6600 \AA$ to $11600 \mathrm{~A}$ with this source. A description of this source was given by Sugar [15]. These spectrograms were presented to Meggers who found that below $8000 \AA$ they were practically identical with those from the Thompson tube, but beyond $9000 \AA$ they added several hundred lines to our list, many of which have been explained as transitions between previously established Yb II energy levels. Some ytterbium lines appear slightly hazy $(\mathrm{h})$ in the sliding spark.

A major contribution to this description of ytterbium spectra is found in the data on Zeeman effect. As noted in the introduction, the first Zeeman patterns 
of ytterbium lines were obtained accidentally in 1939 when they appeared as impurities in a thulium sample under investigation at MIT. Those Zeeman spectrograms were made with a d-c arc in the Bitter magnet [16] with field intensities at 7.30, 8.50, and 9.358 tesla; both factors favored ionization so that Zeeman patterns were recorded only for strong lines of $\mathrm{Yb}$ II or $\mathrm{Yb}$ III and none for YbI. The total number of ytterbium lines with Zeeman patterns on MiT spectrograms was about 400 , and in favorable cases the resolution reached 0.05 Lorentz unit, but most of the lines lay between $2200 \AA$ and $4300 \AA$.

To extend and supplement the Zeeman data for ytterbium obtained at MIT in 1939, we resumed investigations in 1959 at the National Bureau of Standards where a Weiss magnet (with ferro-cobalt pole pieces) was used. When that magnet was animated with $15 \mathrm{~kW}$, it produced a field intensity of $3.70 \mathrm{~T}$ in a pole gap of $5 \mathrm{~mm}$. Special electrodeless lamps containing pure ytterbium metal (and argon gas) were made of quartz tubing $4 \mathrm{~mm}$ o.d. to be inserted and excited between the pole pieces of the magnet. Care was taken to vaporize the metal only in that portion of the lamp located in the region of uniform magnetic field between the pole pieces. The lamp was viewed end-on and focused on the slit of the spectrograph containing a concave grating with 30,000 groves per inch, the same one used in the first and second orders to describe ytterbium spectra between $2000 \AA$ and $9000 \AA$. A Wollaston prism was inserted on the optical axis; it produced a vertical separation of polarized components of Zeeman patterns (parallel or normal to the lines of force) and, since the spectrograph was stigmatic, it recorded all components simultaneously without mixing any. Indeed, the Wollaston was placed where it separated the polarized components sufficiently to insert a narrow strip of "no field" exposure to the ytterbium spectra, useful for recognizing asymmetrical or overlapping Zeeman patterns, and for assigning unresolved patterns to their proper spectrum by noting the reduced intensity of the first spectrum relative to the second in the magnetic field. The magnetic field intensity was calibrated with standard splittings of ubiquitous impurity lines, principally of calcium, magnesium, sodium, and silicon. In this series of Zeeman-effect observations, the highest resolution achieved was about 0.10 Lorentz unit. The first results of Zeeman patterns in $\mathrm{Yb} I$ were announced by Meggers and Corliss [17] in 1960.

\section{Results}

These investigations, extending intermittently over a quarter century, resulted in the condensed data displayed in table 1 , in which successive columns contain measured wavelengths (in air), estimated relative intensities and other attributes in two or more sources, successive spectra assignments, and types of Zeeman patterns.

In general, the wavelengths are the average of two to four or five measurements, and are regarded re- liable to $0.01 \AA$ in most cases. In the range $2500 \AA$ to $4500 \AA$, photographed with our highest dispersion, most strong lines were accompanied by two or more Rowland ghosts. In such cases, the ghosts contributed to the wavelength determination of the parent line, but were scratched from the final list. When the average deviation from the mean of several values was less than $0.005 \AA$, the third decimal was retained.

Because of the electronic structures of ytterbium atoms and ions (deduced from their spectra), their spectra are characterized by an extraordinary range of line intensities. In table 1 , these are roughly estimated from photographic images of illuminated slits and assigned numerical values from 1 to 100,000 . In spite of their crudeness, these estimates of relative intensities in the same and in different light sources provide general criteria for assigning lines to their proper spectrum ( $\mathrm{YbI}$ or $\mathrm{YbII}$ ) and in the same spectrum they often distinguish lines of different excitation. Yb III and Yb IV lines were not excited in microwave discharges but could be seen in spectra of the Thompson tube where they were distinguished by radial intensity gradients. Literal symbols following intensity numbers have the following meanings:

$$
\begin{aligned}
& \mathrm{A}=\text { nearly all absorbed in the pulsed arc. } \\
& \mathrm{R}=\text { wide reversal in the pulsed arc. } \\
& r=\text { narrow reversal in the pulsed arc. } \\
& d=\text { double but not resolved. } \\
& e=\text { enhanced near wall of Thompson tube. } \\
& h=\text { hazy as distinguished from sharp. } \\
& \mathrm{H}=\text { very hazy. } \\
& l=\text { shaded or displaced to longer waves. } \\
& s=\text { shaded or displaced to shorter waves. }
\end{aligned}
$$

The above-mentioned estimates of spectral-line intensities and supplementary information on line attributes constitute the main criteria for sorting the observed lines in four successive spectra: Yb I from neutral $\mathrm{Yb}$ atom, $\mathrm{Yb}$ II from $\mathrm{Yb}^{+}$ions, $\mathrm{Yb}$ III from $\mathrm{Yb}^{2+}$ ions, and $\mathrm{Yb} \mathrm{IV}$ from $\mathrm{Yb}^{3+}$ ions. The assignment of observed lines to their proper spectrum is indicated by Roman numerals in column 4 of table 1; there are about 1800 lines for $\mathrm{Yb} \mathrm{I}, 5100$ for $\mathrm{Yb} \mathrm{II}$, 430 for $\mathrm{Yb} \mathrm{III}$, and 5 for $\mathrm{Yb}$ IV.

In the last column of table 1 , we list the Zeemantype numbers for about 1300 lines observed in strong magnetic fields. These numbers correspond to the classification of Back and Landé [18] who showed that only 7 types of Zeeman patterns exist, types 1, 2, 3, for energy levels of odd multiplicity, types 4, 5, 6 for even, and type 7 appearing in either multiplicity as a single undisplaced parallel $(p)$ component and two symmetrically displaced normal $(n)$ components. The Zeeman types in table 1 prove that $\mathrm{Yb}$ I and $\mathrm{Yb}$ III result from energy levels of odd multiplicity, and $\mathrm{Yb}$ II from even. Further details of the Zeeman patterns, including quantum numbers ( $\mathrm{J}$ and $\mathrm{L}$ ) and magnetic splitting factors $(g)$ derived from them, will be presented in subsequent papers dealing with atomic energy levels and quantum interpretations of $\mathrm{Yb}$ I and $\mathrm{Yb}$ II spectra. 
TABLE 1.-Emission spectra of ytterbium

\begin{tabular}{|c|c|c|c|c|c|c|c|c|c|c|c|c|c|c|}
\hline \multirow{3}{*}{$\begin{array}{l}\text { Wave- } \\
\text { length } \\
\text { in air } \\
\circ \\
\text { A }\end{array}$} & \multicolumn{2}{|c|}{ Intensity } & \multirow{3}{*}{ Spectrum } & \multirow{3}{*}{$\begin{array}{c}\text { Zeeman } \\
\text { type }\end{array}$} & \multirow{3}{*}{$\begin{array}{c}\text { Wave- } \\
\text { length } \\
\text { in air } \\
\\
\AA\end{array}$} & \multicolumn{2}{|c|}{ Intensity } & \multirow{3}{*}{ Spectrum } & \multirow{3}{*}{$\begin{array}{c}\text { Zeeman } \\
\text { type }\end{array}$} & \multirow{3}{*}{$\begin{array}{l}\text { Wave- } \\
\text { length } \\
\text { in air } \\
\circ \\
\text { A }\end{array}$} & Inte & nsity & & \\
\hline & Meggers & Thompson & & & & Meggers & Thompson & & & & Meggers & Thompson & Jpecing & type \\
\hline & lamp & lamp & & & & lamp & lamp & & & & lamp & lamp & & \\
\hline 11603.94 & 20 & & I & & 10445.27 & & $4 \mathrm{~h}$ & II & & 9970.44 & 5 & & I & \\
\hline 11559.05 & & 5 & II & & 10425,42 & & 2 & II & & 9970.1 & & $200 \mathrm{H}$ & II & \\
\hline 11442.70 & & 1 & II & & 10416.16 & & 5 & II & & 9959.25 & & 2 & II & \\
\hline 11299.78 & & $5 \mathrm{~h}$ & II & & 10414.15 & & 1 & II & & 9953.32 & & 4 & II & \\
\hline 11277.83 & & 1 & II & & 10411.40 & & 10 & II & & 9945.98 & & 4 & II & \\
\hline 11262.27 & 250 & 10 & I & & 10397.98 & & 3 & II & & 9940.23 & & 3 & II & \\
\hline 11060.25 & & $2 \mathrm{~h}$ & II & & 10397.88 & 4 & & I & & 9932.98 & & 1 & II & \\
\hline 11051.05 & & 2 & II & & 10391.64 & & $1 \mathrm{~h}$ & II & & 9915.99 & & 6 & II & \\
\hline 11035.15 & & $1 \mathrm{~h}$ & II & & 10388.85 & & $2 \mathrm{~h}$ & II & & 9914.02 & & 2 & II & \\
\hline 11023.37 & & $1 \mathrm{~h}$ & II & & 10363.89 & & 3 & II & & 9904.5 & & 3 & II & \\
\hline 11002.95 & & 4 & II & & 10345.62 & & $1 \mathrm{~h}$ & II & & 9896.50 & & 20 & II & \\
\hline 10980.47 & & $3 \mathrm{~h}$ & II & & 10343.99 & & $4 \mathrm{~h}$ & II & & 9894.20 & 8 & & 1 & \\
\hline 10959.94 & & $1 \mathrm{~h}$ & II & & 10343.85 & 3 & & I & & 9893.9 & & $100 \mathrm{H}$ & iI & \\
\hline 10943.17 & & 1 & II & & 10341.77 & 7 & & I & & 9892.20 & & $4 \mathrm{~h}$ & II & \\
\hline 10882.92 & & $1 \mathrm{~h}$ & II & & 10339.49 & & $2 \mathrm{~h}$ & II & & 9888.40 & 2 & & 1 & \\
\hline 10856.25 & & 1 & II & & 10337.35 & & $1 \mathrm{~h}$ & II & & 9885.02 & & 1 & II & \\
\hline 10845.53 & & $3 \mathrm{~h}$ & II & & 10328.74 & & $2 \mathrm{~h}$ & II & & 9884.09 & & 3 & II & \\
\hline 10830.36 & & 100 & II & & 10327.33 & & 1 & II & & 9882.33 & 1 & & I & \\
\hline 10829.11 & & 40 & II & & 10321.68 & 500 & 80 & I & & 9881.04 & & 1 & II & \\
\hline 10810.82 & & $5 \mathrm{~h}$ & II & & 10293.40 & & $2 \mathrm{~h}$ & II & & 9870.17 & 200 & 15 & I & \\
\hline 10802.47 & & 4 & II & & 10285.15 & & 2 & II & & 9849.25 & & $2 \mathrm{~h}$ & II & \\
\hline 10776.11 & & 1 & II & & 10283.38 & & 2 & II & & 9837.89 & & $2 \mathrm{~h}$ & II & \\
\hline 10774.38 & & 1 & II & & 10275.82 & & 1 & il & & 9831.13 & 7 & & 1 & \\
\hline 10770.10 & 2000 & 400 & I & & 10267.37 & 300 & 100 & 1 & & 9830.09 & & 2 & II & \\
\hline 10754.99 & & 1 & II & & 10259.93 & & $3 \mathrm{~h}$ & II & & 9826.25 & & 2 & II & \\
\hline 10745.87 & & 3 & II & & 10257.97 & & $1 \mathrm{~h}$ & II & & 9826.14 & 1 & & I & \\
\hline 10731.94 & & 3 & II & & 10251.85 & & 10 & II & & 9823.76 & 9 & & I & \\
\hline 10727.72 & 200 & 20 & I & & 10242.30 & & $1 \mathrm{~h}$ & II & & 9818.33 & & $6 \mathrm{~h}$ & II & \\
\hline 10721.68 & & $3 \mathrm{~h}$ & II & & 10222.98 & & $2 \mathrm{~h}$ & II & & 9817.16 & 1 & & I & \\
\hline 10717.07 & & 2 & II & & 10212.34 & & $1 \mathrm{~h}$ & II & & 9816.25 & & $2 \mathrm{~h}$ & II & \\
\hline 10717.02 & 2 & & 1 & & 10212.27 & 1 & & I & & 9800.41 & & 1 & II & \\
\hline 10711.60 & & $80 \mathrm{~h}$ & II & & 10198.92 & & $1 \mathrm{~h}$ & II & & 9799.96 & 400 & 10 & 1 & \\
\hline 10691.02 & & $1 \mathrm{~h}$ & II & & 10189.53 & & $10 \mathrm{~h}$ & II & & 9797.67 & & 6 & II & \\
\hline 10689.20 & & $2 \mathrm{~h}$ & II & & 10186.03 & & 5 & II & & 9775.49 & 1 & & 1 & \\
\hline 10678.64 & & 1 & II & & 10172.39 & & 2 & II & & 9770.61 & & $2 \mathrm{~h}$ & II & \\
\hline 10676.73 & & 30 & II & & 10168.71 & & $1 \mathrm{~h}$ & II & & 9760.38 & 200 & 1000 & II & \\
\hline 10666.30 & & $2 \mathrm{~h}$ & II & & 10167.42 & & $1 \mathrm{~h}$ & II & & 9745.39 & & 7 & II & \\
\hline 10651.36 & & 5 & II & & 10157.63 & & 1 & II & & 9735.10 & & 1 & II & \\
\hline 10638.35 & & 1 & II & & 10148.91 & & 3 & II & & 9734.62 & 2 & & 1 & \\
\hline 10633.24 & 5 & & I & & 10145.30 & & $4 \mathrm{~h}$ & II & & 9718.81 & 3 & & I & \\
\hline 10632.89 & & $400 \mathrm{~h}$ & I & & 10136.34 & & $3 \mathrm{~h}$ & II & & 9717.58 & & 2 & II & \\
\hline 10628.11 & & 4 & II & & 10110.87 & 10 & & I & & 9716.88 & & 8 & II & \\
\hline 10602.75 & & $2 \mathrm{~h}$ & II & & 10110.60 & & 200 & III & & 9711.44 & & 5 & II & \\
\hline 10584.87 & & 1 & II & & 10107.09 & & 1 & II & & 9706.65 & & 10 & II & \\
\hline 10580.74 & & 1 & II & & 10103.24 & & 3 & II & & 9705.60 & & $3 \mathrm{~h}$ & II & \\
\hline 10574.50 & & $2 \mathrm{~h}$ & II & & 10100.39 & & $1 \mathrm{~h}$ & II & & 9700.26 & & $2 \mathrm{~h}$ & II & \\
\hline 10570.51 & & 1 & II & & 10086.67 & & 1 & II & & 9695.71 & 2 & & I & \\
\hline 10567.5 & 1 & & I & & 10068.79 & & $2 \mathrm{~h}$ & II & & 9694.33 & & 2 & II & \\
\hline 10567.42 & & 3 & II & & 10067.47 & & 1 & II & & 9693.72 & & 50 & II & \\
\hline 10565.46 & & 4 & II & & 10066.30 & & 5 & II & & 9688.77 & 25 & & I & \\
\hline 10556.48 & & $2 \mathrm{~h}$ & II & & 10063.22 & & $2 \mathrm{~h}$ & II & & 9678.00 & 1 & 5 & II & \\
\hline 10547.78 & & 4 & II & & 10046.95 & & 2 & II & & 9677.56 & & 1 & II & \\
\hline 10546.24 & & 1 & II & & 10039.90 & & 1 & II & & 9671.78 & & 1 & II & \\
\hline 10516.65 & & 40 & II & & 10032.55 & & 50 & II & & 9670.80 & 5 & & I & \\
\hline 10516.61 & 60 & & I & & 10020.70 & & 1 & II & & 9669.62 & & 1 & II & \\
\hline 10502.56 & & 1 & II & & 10016.78 & & 15 & II & & 9669.03 & & 2 & II & \\
\hline 10501.08 & & 4 & II & & 10007.96 & & 3 & II & & 9664.23 & & 20 & II & \\
\hline 10490.03 & & 1 & II & & 10003.38 & & 4 & II & & 9663.60 & & 1 & II & \\
\hline 10485.56 & & 2 & II & & 9994.64 & & 1 & II & & 9663.09 & & 2 & II & \\
\hline 10477.15 & & $1 \mathrm{~h}$ & II & & 9992.75 & & 1 & II & & 9661.01 & & $6 \mathrm{~h}$ & II & \\
\hline 10456.88 & & 1 & II & & 9990.47 & & 6 & II & & 9659.43 & 2 & & 1 & \\
\hline 10455.88 & & 2 & II & & 9984.66 & & 1 & II & & 9657.25 & & 3 & II & \\
\hline 10446.15 & & 1 & II & & 9976.50 & 2 & 30 & II & & 9656.05 & 20 & & I & \\
\hline
\end{tabular}


TABLE 1.-Emission spectra of ytterbium-Continued

\begin{tabular}{|c|c|c|c|c|c|c|c|c|c|c|c|c|c|c|}
\hline \multirow{3}{*}{$\begin{array}{l}\text { Wave- } \\
\text { length } \\
\text { in air }\end{array}$} & \multicolumn{2}{|c|}{ Intensity } & \multirow{3}{*}{ Spectrum } & \multirow{3}{*}{$\begin{array}{c}\text { Zeeman } \\
\text { type }\end{array}$} & \multirow{3}{*}{$\begin{array}{l}\text { Wave- } \\
\text { length } \\
\text { in air } \\
\AA \\
\end{array}$} & \multicolumn{2}{|c|}{ Intensity } & \multirow{3}{*}{ Spectrum } & \multirow{3}{*}{$\begin{array}{c}\text { Zeeman } \\
\text { type }\end{array}$} & \multirow{3}{*}{$\begin{array}{l}\text { Wave- } \\
\text { length } \\
\text { in air } \\
\circ \\
\end{array}$} & Inte & nsity & & \\
\hline & Meggers & Thompson & & & & Meggers & Thompson & & & & Meggers & Thompson & spectrom & type \\
\hline & lamp & lamp & & & & lamp & lamp & & & & lamp & lamp & & \\
\hline 9648.86 & & 3 & II & & 9377.90 & 7 & & I & & 9155.30 & 40 & & I & \\
\hline 9644.92 & & $2 \mathrm{~h}$ & II & & 9372.12 & & 3 & II & & 9152.45 & & $8 \mathrm{H}$ & II & \\
\hline 9640.78 & 2 & & I & & 9367.86 & & 1 & II & & 9150.60 & & $1 \mathrm{~h}$ & II & \\
\hline 9637.59 & & $3 \mathrm{~h}$ & II & & 9367.21 & & 2 & II & & 9149.57 & & $2 \mathrm{~h}$ & II & \\
\hline 9630.97 & & 5 & II & & 9352.58 & 8 & 80 & II & & 9142.22 & & 7 & II & \\
\hline 9629.04 & & 2 & II & & 9349.25 & 80 & 300 & II & & 9141.37 & & 15 & II & \\
\hline 9623.60 & & $9 \mathrm{~h}$ & II & & 9341.89 & & $4 \mathrm{~h}$ & II & & 9140.64 & 10 & & I & \\
\hline 9619.82 & & $3 \mathrm{~h}$ & II & & 9334.67 & & 3 & II & & 9133.17 & 3 & 60 & II & \\
\hline 9613.49 & & 1 & II & & 9328.56 & & $3 \mathrm{~h}$ & II & & 9128.47 & 20 & & I & \\
\hline 9611.30 & & 2 & II & & 9325.68 & & $20 \mathrm{~h}$ & II & & 9127.08 & & 1 & II & \\
\hline 9610.77 & & 2 & II & & 9324.60 & & $2 \mathrm{~h}$ & II & & 9126.00 & & $7 \mathrm{~h}$ & II & \\
\hline 9608.81 & & 2 & II & & 9321.14 & & $2 \mathrm{~h}$ & II & & 9123.62 & & 2 & II & \\
\hline 9606.08 & 3 & & I & & 9320.30 & & 1 & II & & 9122.30 & & 15 & II & \\
\hline 9602.64 & & 20 & II & & 9316.58 & & 1 & II & & 9121.10 & 3 & & I & \\
\hline 9600.55 & & $2 \mathrm{~h}$ & II & & 9314.88 & 2 & 40 & II & & 9120.52 & & $5 \mathrm{~h}$ & II & \\
\hline 9595.60 & & $2 \mathrm{~h}$ & II & & 9304.35 & 1000 & 80 & I & & 9117.68 & 100 & 4 & I & \\
\hline 9594.26 & & $2 \mathrm{~h}$ & II & & 9302.74 & & 2 & II & & 9113.24 & & $7 \mathrm{~h}$ & II & \\
\hline 9592.23 & 1 & & I & & 9301.33 & & 3 & II & & 9105.95 & & $15 \mathrm{~h}$ & II & \\
\hline 9589.34 & 1 & & I & & 9299.67 & & 1 & II & & 9104.10 & 200 & 7 & I & \\
\hline 9589.14 & & $7 \mathrm{~h}$ & II & & 9298.90 & 3 & & I & & 9097.69 & 2 & & I & \\
\hline 9580.31 & 30 & & I & & 9298.74 & & 6 & II & & 9096.88 & & 20 & II & \\
\hline 9578.21 & & 1 & II & & 9291.74 & 2 & & I & & 9094.57 & & 4 & II & \\
\hline 9577.45 & & 3 & II & & 9289.87 & 4 & 100 & II & & 9089.55 & $2 \mathrm{~d}$ & & I & \\
\hline 9572.65 & 5 & & I & & 9287.36 & & 1 & II & & 9083.98 & & $3 \mathrm{~h}$ & II & \\
\hline 9564.64 & & 3 & II & & 9272.68 & 3 & 30 & II & & 9082.61 & & 7 & II & \\
\hline 9559.83 & & 5 & II & & 9271.84 & & 2 & II & & 9080.35 & 1 & 60 & II & \\
\hline 9555.55 & & 5 & II & & 9263.73 & & $4 \mathrm{~h}$ & II & & 9077.91 & & 4 & II & \\
\hline 9536.43 & & 3 & II & & 9261.11 & & $4 \mathrm{~h}$ & II & & 9076.21 & & 2 & II & \\
\hline 9532.71 & & $8 \mathrm{~h}$ & II & & 9258.76 & 1 & & I & & 9073.18 & 1 & & I & \\
\hline 9532.64 & 1 & & I & & 9257.70 & & 8 & II & & 9069.22 & 20 & & I & \\
\hline 9531.83 & & 20 & II & & 9253.57 & 7 & 70 & II & & 9066.47 & & 1 & II & \\
\hline 9527.91 & & $3 \mathrm{~h}$ & II & & 9252.27 & & 3 & II & & 9062.06 & & 15 & II & \\
\hline 9524.36 & 300 & 6 & I & & 9251.95 & 1 & 5 & II & & 9057.08 & & $3 \mathrm{~h}$ & II & \\
\hline 9522.68 & & $4 \mathrm{~h}$ & II & & 9245.69 & & $2 \mathrm{~h}$ & II & & 9055.35 & & 2 & II & \\
\hline 9520.19 & & 3 & II & & 9241.86 & & 2 & II & & 9045.47 & 1 & & I & \\
\hline 9512.32 & 2 & & I & & 9230.17 & 7 & & I & & 9043.95 & 5 & & I & \\
\hline 9511.61 & & 3 & II & & 9226.17 & & $6 \mathrm{~h}$ & II & & 9040.38 & & $3 \mathrm{~h}$ & II & \\
\hline 9505.71 & 2 & & I & & 9224.21 & 6 & & I & & 9039.08 & 1 & & I & \\
\hline 9504.03 & & 1 & II & & 9220.77 & & $7 \mathrm{~h}$ & II & & 9035.02 & 2 & 20 & II & \\
\hline 9498.90 & & 3 & II & & 9218.26 & & 1 & II & & 9029.62 & & 2 & II & \\
\hline 9496.63 & & 10 & II & & 9217.40 & & 6 & II & & 9021.00 & & $100 \mathrm{~h} \mathrm{l}$ & II & \\
\hline 9490.85 & & 2 & II & & 9215.28 & & 5 & II & & 9020.90 & 10 & & I & \\
\hline 9482.44 & & 20 & II & & 9211.07 & & 2 & II & & 9017.02 & & $5 \mathrm{~h}$ & II & \\
\hline 9475.35 & & 1 & II & & 9207.65 & & $7 \mathrm{~h}$ & II & & 9016.21 & 2 & & I & \\
\hline 9474.20 & & $2 \mathrm{~h}$ & II & & 9196.5 & & $20 \mathrm{H}$ & II & & 9014.27 & & 2 & II & \\
\hline 9468.54 & 2 & 3 & I,II & & 9192.40 & & $3 \mathrm{~h}$ & II & & 9004.83 & & 3 & II & \\
\hline 9468.20 & & $10 \mathrm{~h}$ & II & & 9191.41 & & 3 & II & & 9004.21 & 10 & 150 & II & \\
\hline 9464.03 & & $4 \mathrm{~h}$ & II & & 9190.18 & & $5 \mathrm{~h}$ & II & & 8997.66 & 400 & 10 & I & \\
\hline 9457.07 & & $6 \mathrm{~h}$ & II & & 9188.34 & 10 & & I & & 8985.71 & 8 & 60 & II & \\
\hline 9445.45 & & $6 \mathrm{~h}$ & II & & 9187.53 & & 4 & II & & 8982.45 & & 2 & II & \\
\hline 9442.75 & & 7 & II & & 9187.01 & & 1 & II & & 8980.79 & 3 & & I & \\
\hline 9428.10 & & 30 & II & & 9182.32 & & $5 \mathrm{~h}$ & II & & 8980.74 & & 20 & II & \\
\hline 9423.79 & & $3 \mathrm{~d}$ & II & & 9178.52 & & 5 & II & & 8978.58 & & 6 & II & \\
\hline 9417.09 & & 5 & II & & 9177.47 & 1 & & I & & 8971.65 & & $3 \mathrm{~h}$ & II & \\
\hline 9408.11 & & 4 & II & & 9176.14 & 1 & & I & & 8967.71 & & 5 & II & \\
\hline 9405.75 & & 3 & II & & 9175.76 & & $2 \mathrm{~h}$ & II & & 8959.06 & 2 & 70 & II & \\
\hline 9404.73 & & 10 & II & & 9172.59 & & $2 \mathrm{~h}$ & II & & 8956.72 & & 9 & II & \\
\hline 9397.79 & & $2 \mathrm{~h}$ & II & & 9170.70 & & $3 \mathrm{~h}$ & II & & 8952.23 & & 6 & II & \\
\hline 9390.61 & & $8 \mathrm{~h}$ & II & & 9168.70 & & $2 \mathrm{~h}$ & II & & 8948.02 & 2 & & I & \\
\hline 9388.32 & & 2 & II & & 9167.06 & 3 & 40 & II & & 8947.78 & & 6 & II & \\
\hline 9382.31 & 2 & & I & & 9163.69 & & 15 & II & & 8946.66 & & 5 & II & \\
\hline 9380.18 & & 15 & II & & 9162.85 & & 2 & II & & 8946.38 & 1 & & I & \\
\hline 9378.29 & & 3 & II & & 9158.62 & & 15 & II & & 8942.87 & & 3 & II & \\
\hline
\end{tabular}


TABLE 1.-Emission spectra of ytterbium-Continued

\begin{tabular}{|c|c|c|c|c|c|c|c|c|c|c|c|c|c|c|}
\hline \multirow{3}{*}{$\begin{array}{c}\text { Wave- } \\
\text { length } \\
\text { in air }\end{array}$} & \multicolumn{2}{|c|}{ Intensity } & \multirow{3}{*}{ Spectrum } & \multirow{3}{*}{$\begin{array}{c}\text { Zeeman } \\
\text { type }\end{array}$} & \multirow{3}{*}{$\begin{array}{c}\text { Wave- } \\
\text { length } \\
\text { in air }\end{array}$} & \multicolumn{2}{|c|}{ Intensity } & \multirow{3}{*}{ Spectrum } & \multirow{3}{*}{$\begin{array}{c}\text { Zeeman } \\
\text { type }\end{array}$} & \multirow{3}{*}{$\begin{array}{c}\text { Wave- } \\
\text { length } \\
\text { in air }\end{array}$} & & nsity & & \\
\hline & Meggers & Thompson & & & & Meggers & Thompson & & & & Meggers & Thompson & & type \\
\hline & lamp & lamp & & & & lamp & lamp & & & & lamp & lamp & & \\
\hline 8930.12 & & 1 & II & & 8710́.15 & & 4 & II & 4 & 8518.01 & 20 & & I & \\
\hline 8927.38 & & 4 & II & & 8699.84 & & 1 & II & & 8517.54 & & $2 \mathrm{~h}$ & II & \\
\hline 8922.56 & & $100 \mathrm{~h}$ & II & & 8697.67 & & 1 & II & & 8516.60 & 2 & & I & \\
\hline 8922.50 & 1000 & 40 & I & & 8691.24 & & 1 & II & & 8515.78 & 3 & & I & \\
\hline 8912.14 & 4 & 60 & II & & 8686.28 & 2 & & I & & 8515.24 & 2 & & I & \\
\hline 8907.81 & & $2 \mathrm{~h}$ & II & & 8681.93 & 3 & & I & & 8508.02 & 10 & & I & \\
\hline 8902.99 & 2 & & I & & 8679.50 & 2 & & I & & 8505.96 & 2 & & I & \\
\hline 8902.92 & & $7 \mathrm{~h}$ & II & & 8672.34 & & 5 & II & 7 & 8503.56 & & $1 \mathrm{~h}$ & II & \\
\hline 8898.89 & 1 & & I & & 8671.53 & & 1 & II & & 8502.18 & & 3 & II & 4 \\
\hline 8897.61 & & 1 & II & & 8670.82 & 2 & & I & & 8500.55 & & 2 & II & \\
\hline 8896.50 & & 4 & II & & 8667.69 & 50 & & I & & 8494.37 & 50 & & I & \\
\hline 8890.45 & 5 & 50 & II & & 8663.92 & 10 & & I & & 8493.74 & 4 & & I & \\
\hline 8888.66 & 3 & & I & & 8663.39 & & $4 \mathrm{~h}$ & III & & 8489.90 & & $30 \mathrm{e}$ & III & \\
\hline 8883.16 & 10 & & I & & 8661.59 & 5 & & I & & 8487.84 & 1 & & I & \\
\hline 8874.68 & 2 & & I & & 8659.04 & & 15 & II & 4 & 8485.10 & 30 & & I & \\
\hline 8874.53 & & 9 & II & & 8655.52 & $15 \mathrm{~h}$ & & I & & 8483.31 & & 2 & II & \\
\hline 8873.33 & & 4 & II & & 8654.91 & 100 & & I & & 8481.03 & & 2 & II & \\
\hline 8867.40 & & 1 & II & & 8654.42 & 20 & & I & & 8480.27 & & $2 \mathrm{~h}$ & II & \\
\hline 8865.65 & & 1 & II & & 8650.23 & 2 & & I & & 8477.79 & & $2 \mathrm{~h}$ & II & \\
\hline 8864.18 & & 1 & II & & 8641.86 & & 15 & II & & 8474.21 & 3 & & I & \\
\hline 8864.06 & 2 & & I & & 8634.68 & 9 & & I & & 8473.35 & & 2 & II & \\
\hline 8863.98 & & 4 & II & & 8633.25 & & 1 & II & & 8465.75 & & 15 & II & 6 \\
\hline 8862.12 & & 3 & II & & 8621.62 & & 2 & II & 6 & 8463.32 & 1 & & I & \\
\hline 8861.70 & & 1 & II & & 8620.75 & & 1 & II & & 8462.25 & 4 & & 1 & \\
\hline 8855.12 & 50 & 300 & II & & 8612.57 & 50 & & I & & 8460.54 & & 2 & II & \\
\hline 8851.05 & & 1 & II & & 8607.49 & 200 & 400 & II & 6 & 8458.72 & & 1 & II & \\
\hline 8849.52 & & 1 & II & & 8603.56 & 80 & & 1 & & 8455.87 & 4 & 30 & II & 4 \\
\hline 8848.83 & 4 & 60 & II & & 8601.25 & 1 & & I & & 8446.74 & 5 & & I & \\
\hline $\begin{array}{l}0040.03 \\
8842.64\end{array}$ & 4 & $\begin{array}{r}00 \\
2\end{array}$ & II & & 8597.22 & 1 & $2 \mathrm{~h}$ & III & & 8446.35 & 20 & & I & \\
\hline 8839.24 & & 10 & II & & 8594.75 & 2 & & I & & 8446.32 & & 6 & II & \\
\hline 8831.38 & 2 & & I & & 8594.57 & & 8 & II & & 8443.99 & 5 & & I & \\
\hline 8829.26 & 30 & & I & & 8592.85 & & 10 & II & 5 & 8438.92 & & 1 & II & \\
\hline 8827.46 & 1 & & I & & 8592.00 & 200 & & I & & 8438.42 & & 2 & II & \\
\hline 8825.52 & & 2 & II & & 8591.26 & 20 & & I & & 8437.07 & & 3 & II & \\
\hline 8822.45 & & 7 & II & & 8590.94 & 20 & & I & & 8435.61 & & 3 & II & \\
\hline 8815.52 & & 9 & II & & 8590.08 & 1 & & I & & 8430.76 & 2 & & 1 & \\
\hline 8806.80 & 6 & & I & & 8587.89 & & 1 & II & & 8429.59 & 3 & & I & \\
\hline 8806.05 & & 2 & II & & 8585.99 & 20 & & I & & 8428.83 & & 15 & II & 1 \\
\hline 8800.32 & & $2 \mathrm{~h}$ & II & & 8585.00 & & 1 & II & & 8428.24 & 10 & & I & \\
\hline 8797.99 & 5 & 100 & II & & 8584.20 & 2 & & I & & 8427.26 & & 3 & II & \\
\hline 8794.91 & 5 & & I & & 8579.08 & & 7 & II & 6 & 8424.89 & & 1 & II & \\
\hline 8788.45 & 5 & & I & & 8575.25 & 10 & & I & & 8423.84 & 1 & & I & \\
\hline 8783.76 & & 2 & II & & 8574.91 & 2 & & I & & 8418.43 & 10 & & I & \\
\hline 8783.75 & 4 & & I & & 8566.83 & & 2 & II & & 8416.53 & 20 & & I & \\
\hline 8781.96 & 1 & 10 & II & 6 & 8561.74 & & 20 & II & 6 & 8411.84 & 1 & 100 & II & 4 \\
\hline 8780.63 & 10 & & I & & 8558.47 & $2 \mathrm{~h}$ & & I & & 8408.89 & & 3 & II & \\
\hline $\begin{array}{l}8180.03 \\
8762.42\end{array}$ & 10 & 5 & II & 5 & $\begin{array}{l}\text { O50.46 } \\
8556.90\end{array}$ & 211 & 4 & II & & 8403.58 & & 2 & II & \\
\hline 8761.26 & & 2 & II & & 8554.92 & & 2 & II & 6 & 8400.65 & 20 & & I & \\
\hline 8750.88 & & $1 \mathrm{~h}$ & II & & 8548.15 & & 1 & II & & 8400.61 & & 1 & II & \\
\hline 8749.10 & & 40 & II & 4 & 8546.26 & 40 & & I & & 8400.35 & 5 & & I & \\
\hline 8744.27 & & 1 & II & & 8543.66 & & 5 & II & & 8400.01 & & 20 & III & \\
\hline 8742.84 & & 7 & II & & 8535.68 & 20 & & I & & 8396.79 & & 8 & II & 4 \\
\hline 8742.45 & & 10 & II & 7 & 8532.59 & & 1 & II & & 8392.00 & & $4 \mathrm{~h}$ & III & \\
\hline 8740.48 & & 5 & II & & 8528.08 & 10 & & I & & 8391.62 & 3 & & I & \\
\hline 8737.78 & & 10 & II & 7 & 8527.65 & & 1 & II & & 8383.90 & 2 & & I & \\
\hline 8736.45 & 20 & & I & & 8525.66 & 3 & & I & & 8381.26 & & 20 & II & \\
\hline 8736.03 & 4 & & I & & 8525.54 & & 1 & II & & 8380.03 & & 3 & II & \\
\hline 8735.23 & & 1 & II & & 8524.07 & 3 & & I & & 8379.39 & & 8 & II & \\
\hline 8731.29 & 30 & & I & & 8523.93 & $5 \mathrm{~h}$ & & I & & 8378.93 & 6 & & I & \\
\hline 8725.19 & & 10 & II & & 8522.92 & 2 & & I & & 8378.34 & & 3 & II & \\
\hline 8724.18 & 4 & & I & & 8520.34 & 50 & & I & & 8376.33 & 2 & & I & \\
\hline 8723.33 & & 1 & II & & 8520.12 & 7 & & I & & 8375.94 & 40 & & I & \\
\hline 8717.80 & 1 & & & & 8519.66 & 20 & & & & 8374.40 & & 15 & II & 4 \\
\hline
\end{tabular}


TABLE 1.-Emission spectra of ytterbium-Continued

\begin{tabular}{|c|c|c|c|c|c|c|c|c|c|c|c|c|c|c|}
\hline \multirow{3}{*}{$\begin{array}{c}\text { Wave- } \\
\text { length } \\
\text { in air }\end{array}$} & \multicolumn{2}{|c|}{ Intensity } & \multirow{3}{*}{ Spectrum } & \multirow{3}{*}{$\begin{array}{c}\text { Zeeman } \\
\text { type }\end{array}$} & \multirow{3}{*}{$\begin{array}{c}\begin{array}{c}\text { Wave- } \\
\text { length } \\
\text { in air }\end{array} \\
\AA \\
\end{array}$} & \multicolumn{2}{|c|}{ Intensity } & \multirow{3}{*}{ Spectrum } & \multirow{3}{*}{$\begin{array}{c}\text { Zeeman } \\
\text { type }\end{array}$} & \multirow{3}{*}{$\begin{array}{c}\text { Wave- } \\
\text { length } \\
\text { in air }\end{array}$} & Inte & ensity & & \\
\hline & Meggers & Thompson & & & & Meggers & Thompson & & & & Meggers & Thompson & & type \\
\hline & lamp & lamp & & & & lamp & lamp & & & & lamp & lamp & & \\
\hline 8371.77 & & $2 \mathrm{~h}$ & II & & 8225.59 & $1 \mathrm{~h}$ & & I & & 8115.46 & & 6 & II & \\
\hline 8360.09 & 2 & & I & & 8225.41 & & 5 & II & 6 & 8114.26 & 9 & & I & \\
\hline 8356.75 & & 5 & II & & 8224.13 & 10 & & I & & 8111.71 & 90 & & I & \\
\hline 8352.37 & & 4 & II & & 8221.74 & 10 & & I & & 8111.61 & & 9 & II & \\
\hline 8352.03 & 2 & & I & & 8221.35 & 10 & & I & & 8111.19 & 2 & 10 & II & 6 \\
\hline 8350.15 & & 9 & II & & 8220.44 & 2 & & I & & 8109.79 & 100 & & I & \\
\hline 8349.52 & $2 \mathrm{~h}$ & & I & & 8213.96 & 9 & & I & & 8104.44 & & 5 & II & 4 \\
\hline 8347.14 & & $2 \mathrm{~h}$ & II & & 8213.64 & & 30 & II & 6 & 8103.22 & & 1 & II & \\
\hline 8346.11 & $3 \mathrm{~h}$ & & I & & 8213.24 & & 10 & II & 6 & 8101.64 & & 1 & II & \\
\hline 8343.49 & & 2 & II & & 8213.02 & 30 & & I & & 8100.20 & & $3 \mathrm{~h}$ & II & \\
\hline 8342.51 & & 8 & II & & 8212.03 & 5 & & I & & 8098.66 & 2 & & I & \\
\hline 8341.30 & & 2 & II & & 8210.33 & 4 & & I & & 8097.06 & & 1 & II & \\
\hline 8336.34 & & 5 & II & & 8207.98 & 40 & & I & & 8095.74 & 1 & & I & \\
\hline 8335.12 & 1 & & I & & 8206.55 & & 3 & II & & 8093.03 & & 8 & II & 6 \\
\hline 8334.43 & & 10 & II & & 8200.20 & 1 & & I & & 8092.95 & 3 & & I & \\
\hline 8333.30 & 10 & & I & & 8199.08 & & 1 & II & & 8091.73 & 1000 & 10 & I & 1 \\
\hline 8331.71 & & $2 \mathrm{~h}$ & II & & 8197.43 & 6 & & I & & 8089.66 & 7 & & I & \\
\hline 8327.88 & & $30 \mathrm{e}$ & III & & 8194.83 & & 2 & II & & 8086.68 & 10 & & I & \\
\hline 8327.71 & 3 & & I & & 8192.40 & & 30 & II & 4 & 8085.56 & 4 & & I & \\
\hline 8326.86 & & $10 \mathrm{e}$ & III & & 8190.61 & & 10 & II & 7 & 8084.74 & & 9 & II & 4 \\
\hline 8325.18 & 800 & 5 & I & 3 & 8190.31 & 5 & & I & & 8084.50 & 1 & & I & \\
\hline 8320.33 & 8 & & I & & 8189.53 & & 2 & II & & 8079.47 & & 5 & II & 6 \\
\hline 8318.01 & & 1 & II & & 8183.28 & & 1 & II & & 8078.10 & & 2 & II & \\
\hline 8317.43 & 20 & & I & & 8180.75 & 10 & & I & & 8076.95 & & 1 & II & \\
\hline 8310.61 & 3 & & I & & 8180.64 & 30 & & I & & 8075.92 & 2 & 40 & II & 6 \\
\hline 8310.53 & & 2 & II & 7 & 8180.34 & & $2 \mathrm{~h}$ & II & & 8074.53 & & 7 & II & 5 \\
\hline 8310.38 & & 2 & II & & 8179.01 & & 4 & II & & 8071.28 & 10 & & I & \\
\hline 8309.10 & 2 & & I & & 8178.46 & & 4 & II & & 8070.00 & 50 & & I & \\
\hline 8308.40 & & 1 & II & & 8178.14 & & 3 & II & & 8067.21 & 7 & & I & \\
\hline 8307.67 & & 2 & II & & 8177.37 & & $2 \mathrm{e}$ & III & & 8066.72 & 2 & 50 & II & 6 \\
\hline 8306.38 & 2 & & I & & 8173.60 & & $3 \mathrm{~h}$ & II & & 8066.43 & 7 & & I & \\
\hline 8301.74 & & 15 & II & 6 & 8171.82 & 1 & 30 & II & 5 & 8066.17 & & $9 \mathrm{e}$ & III & \\
\hline 8299.77 & 2 & & I & & 8169.69 & & $1 \mathrm{~h}$ & II & & 8063.02 & 2 & 60 & II & 4 \\
\hline 8296.83 & 4 & & I & & 8169.29 & & $2 \mathrm{~h}$ & II & & 8058.91 & 80 & & I & \\
\hline 8294.85 & 150 & & I & & 8166.73 & & $4 \mathrm{~h}$ & II & & 8056.20 & 10 & & I & \\
\hline 8291.01 & & 20 & II & & 8166.05 & & 1 & II & & 8056.02 & & $20 \mathrm{e}$ & III & \\
\hline 8289.25 & & 3 & II & & 8162.00 & & $2 \mathrm{~h}$ & II & & 8055.12 & 20 & & I & \\
\hline 8283.78 & & 15 & II & 6 & 8158.49 & 5 & 80 & II & 5 & 8053.43 & 50 & 500 & II & 6 \\
\hline 8282.14 & & 2 & II & & 8157.40 & & 150 & II & 6 & 8052.35 & 20 & & I & \\
\hline 8280.60 & & 2 & II & & 8155.12 & & 3 & II & & 8051.48 & 20 & & I & \\
\hline 8276.29 & & $2 \mathrm{~h}$ & II & & 8149.08 & & 15 & II & 6 & 8049.03 & 5 & 30 & II & 6 \\
\hline 8267.90 & & 2 & II & & 8148.83 & & 3 & II & & 8042.91 & 4 & & I & \\
\hline 8267.87 & 10 & & I & & 8146.64 & & 1 & II & & 8042.63 & & 5 & II & \\
\hline 8266.52 & & 7 & II & & 8145.56 & 20 & & I & & 8039.83 & 200 & 2 & I & 1 \\
\hline 8266.06 & 2 & & I & & 8145.36 & 5 & & I & & 8038.16 & & 10 & II & \\
\hline 8265.64 & 2 & & I & & 8143.81 & & $2 \mathrm{~h}$ & II & & 8035.53 & & 4 & II & 5 \\
\hline 8263.64 & 4 & 150 & II & 4 & 8142.47 & & 20 & II & 4 & 8034.43 & & 1 & II & \\
\hline 8262.66 & & 3 & III & & 8140.67 & & $6 \mathrm{e}$ & III & & 8034.08 & & 2 & III & \\
\hline 8261.70 & & 1 & II & & 8140.53 & 2 & & I & & 8023.46 & 1 & 20 & II & \\
\hline 8260.66 & & 3 & III & & 8139.18 & & $6 \mathrm{e}$ & III & & 8023.11 & 3 & & I & \\
\hline 8259.34 & 1 & & I & & 8137.92 & & 2 & II & & 8022.93 & & 10 & II & \\
\hline 8257.79 & & $7 \mathrm{~h}$ & III & & 8136.38 & 9 & & I & & 8021.62 & 3 & 150 & II & 6 \\
\hline 8249.18 & 50 & & I & & 8133.01 & & 8 & II & & 8018.74 & 3 & & I & \\
\hline 8246.48 & & $1 \mathrm{~h}$ & II & & 8131.92 & & 2 & II & & 8013.17 & & 15 & II & 6 \\
\hline 8242.95 & 1 & & I & & 8124.50 & & 15 & II & & 8009.53 & 4 & 50 & II & 4 \\
\hline 8242.03 & 1 & & I & & 8123.85 & & 9 & II & & 8007.83 & 15 & 300 & II & 4 \\
\hline 8241.66 & & 5 & II & & 8122.77 & 1 & & I & & 8002.73 & 1 & 20 & II & 4 \\
\hline 8240.67 & 2 & & I & & 8122.49 & & 5 & II & 4 & 8001.30 & & $2 \mathrm{~h}$ & II & \\
\hline 8240.01 & & 1 & II & & 8121.35 & & $3 \mathrm{~h}$ & II & & 7994.90 & & 30 & II & 4 \\
\hline 8237.20 & & 2 & II & & 8121.12 & & 2 & II & & 7990.35 & 30 & & I & \\
\hline 8234.61 & 15 & & I & & 8118.80 & 5 & 150 & II & 4 & 7984.31 & & 4 & II & \\
\hline 8230.78 & & 1 & II & & 8117.44 & & $10 \mathrm{e}$ & III & & 7971.46 & & $100 \mathrm{e}$ & III & \\
\hline 8227.41 & & 1 & II & & 8116.08 & 10 & & I & & 7968.09 & 1 & 6 & II & \\
\hline
\end{tabular}


TABLE 1.-Emission spectra of ytterbium-Continued

\begin{tabular}{|c|c|c|c|c|c|c|c|c|c|c|c|c|c|c|}
\hline \multirow{3}{*}{$\begin{array}{l}\text { Wave- } \\
\text { length } \\
\text { in air } \\
\circ \\
\AA\end{array}$} & \multicolumn{2}{|c|}{ Intensity } & \multirow{3}{*}{ Spectrum } & \multirow{3}{*}{$\begin{array}{c}\text { Zeeman } \\
\text { type }\end{array}$} & \multirow{3}{*}{$\begin{array}{l}\text { Wave- } \\
\text { length } \\
\text { in air } \\
\AA \\
\end{array}$} & \multicolumn{2}{|c|}{ Intensity } & \multirow{3}{*}{ Spectrum } & \multirow{3}{*}{$\begin{array}{c}\text { Zeeman } \\
\text { type }\end{array}$} & \multirow{3}{*}{$\begin{array}{c}\begin{array}{c}\text { Wave- } \\
\text { length } \\
\text { in air }\end{array} \\
\circ \\
\end{array}$} & Inte & nsity & & \\
\hline & Meggers & Thompson & & & & Meggers & Thompson & & & & Meggers & Thompson & specirom & type \\
\hline & lamp & lamp & & & & lamp & lamp & & & & lamp & lamp & & \\
\hline 7964.12 & 2 & 80 & II & 4 & 7772.78 & & 2 & II & & 7623.44 & & $4 \mathrm{e}$ & III & \\
\hline 7958.80 & & 3 & II & & 7771.01 & 4 & & I & & 7616.50 & & 2 & II & \\
\hline 7958.48 & 2 & 40 & II & 6 & 7767.43 & & 2 & II & & 7616.34 & 3 & & I & \\
\hline 7950.35 & 2 & 60 & II & 6 & 7766.02 & & 2 & II & & 7611.69 & & 15 & II & 5 \\
\hline 7950.01 & & 2 & II & & 7761.45 & 2 & & I & & 7611.28 & 3 & & I & \\
\hline 7947.60 & 1 & 40 & II & 4 & 7760.49 & 5 & & I & & 7607.09 & & 2 & II & \\
\hline 7946.26 & 50 & & I & & 7758.04 & 500 & 20 & I & 7 & 7603.42 & 2 & & I & \\
\hline 7933.25 & 2 & & I & & 7753.91 & 2 & 5 & II & & 7599.13 & & 20 & II & 6 \\
\hline 7930.04 & & 8 & II & 4 & 7753.29 & & 6 & II & & 7598.01 & 2 & & I & \\
\hline 7928.71 & & 7 & II & & 7749.38 & & 1 & II & & 7596.22 & 200 & 1 & I & \\
\hline 7924.65 & 4 & & I & & 7746.98 & $6 \mathrm{~h}$ & & I & & 7595.20 & & 2 & III & \\
\hline 7922.40 & 4000 & 100 & I & 1 & 7745.93 & & 5 & II & 5 & 7588.43 & 8 & & I & \\
\hline 7918.41 & & 3 & III & & 7744.97 & 5 & & I & & 7582.93 & & 40 & II & 4 \\
\hline 7913.30 & & 6 & II & & 7741.94 & & 3 & II & & 7582.90 & 30 & & I & \\
\hline 7912.46 & & 10 & II & & 7741.66 & & 2 & II & 7 & 7581.32 & & $2 \mathrm{e}$ & III & \\
\hline 7910.08 & 10 & & I & & 7741.15 & & 2 & II & & 7579.97 & 1 & 60 & II & 5 \\
\hline 7906.25 & & $2 \mathrm{~h}$ & II & & 7737.59 & 1 & 8 & II & 4 & 7576.94 & & $4 \mathrm{e}$ & III & \\
\hline 7904.82 & 10 & 200 & II & 4 & 7734.53 & 200 & 2 & I & 3 & 7576.41 & 2 & & I & \\
\hline 7899.54 & & 5 & II & 6 & 7732.46 & & $4 \mathrm{~h}$ & II & & 7572.81 & & 5 & II & \\
\hline 7897.90 & & 2 & II & & 7732.35 & & 6 & II & 6 & 7566.65 & & 15 & II & 4 \\
\hline 7896.38 & 100 & & I & & 7719.82 & & 15 & II & & 7561.76 & & 8 e & III & \\
\hline 7895.51 & & $3 \mathrm{~h}$ & III & & 7719.23 & & 40 & II & 6 & 7561.42 & 4 & & I & \\
\hline 7895.08 & 1000 & 50 & I & 2 & 7717.58 & 6 & & I & & 7560.18 & & 1 & II & \\
\hline 7893.10 & & $20 \mathrm{e}$ & III & & 7713.74 & & $4 \mathrm{e}$ & III & & 7556.13 & 1 & 40 & II & 6 \\
\hline 7892.39 & & $80 \mathrm{e}$ & III & & 7707.28 & & $2 \mathrm{~h}$ & III & & 7555.14 & 1 & 60 & II & 4 \\
\hline 7891.93 & & 1 & II & & 7706.29 & $2 \mathrm{~h}$ & & I & & 7547.08 & 5 & & I & \\
\hline 7889.78 & & 3 & III & & 7702.72 & & $2 \mathrm{~h}$ & III & & 7541.56 & & 2 & III & \\
\hline 7885.17 & & 2 & III & & 7699.48 & 20000 & 2000 & I & 1 & 7541.10 & 5 & 80 & II & 4 \\
\hline 7883.96 & 2 & & I & & 7697.62 & & 5 & II & & 7539.25 & & 8 e & III & \\
\hline 7877.06 & 60 & & I & & 7693.21 & & $6 \mathrm{e}$ & III & & 7537.40 & & 1 & II & \\
\hline 7875.15 & 10 & & I & & 7692.75 & $5 \mathrm{~h}$ & & I & & 7533.09 & & 3 & II & \\
\hline 7874.10 & 1 & 20 & II & 5 & 7684.25 & & $4 \mathrm{~h}$ & III & & 7530.78 & & 3 & II & \\
\hline 7865.13 & & 7 & II & 5 & 7683.77 & & 9 & II & 5 & 7529.61 & & 3 & III & \\
\hline 7856.20 & & 4 & II & 4 & 7679.91 & 200 & 1 & I & 1 & 7528.07 & & 8 & II & \\
\hline 7851.06 & & $3 \mathrm{~h}$ & III & & 7678.20 & & 1 & II & & 7527.46 & 10000 & 200 & I & 1 \\
\hline 7849.85 & & 6 & II & & 7676.62 & & 8 & II & 6 & 7522.92 & & $7 \mathrm{e}$ & III & \\
\hline 7848.49 & & 15 & II & 4 & 7666.57 & & 1 & II & & 7522.73 & 200 & 2 & I & \\
\hline 7844.11 & 20 & 2 & I & 6 & 7664.41 & & $70 \mathrm{e}$ & III & & 7520.94 & & 4 & II & \\
\hline 7840.45 & 1 & 40 & II & 4 & 7664.11 & & $2 \mathrm{~h}$ & III & & 7520.46 & & 15 & II & 6 \\
\hline 7839.17 & & $1 \mathrm{~h}$ & II & & 7662.08 & 2 & & I & & 7517.94 & & 2 & II & \\
\hline 7834.65 & 15 & & I & & 7659.90 & 20 & & I & & 7516.24 & & 2 & II & \\
\hline 7833.02 & & 9 & II & 4 & 7659.40 & & $3 \mathrm{~h}$ & III & & 7513.50 & & $4 \mathrm{e}$ & III & \\
\hline 7825.52 & 1 & 50 & II & 6 & 7659.14 & 50 & & I & & 7512.86 & & 15 & II & \\
\hline 7824.64 & & 3 & II & & 7657.60 & 100 & & I & & 7508.07 & & 8 & II & 5 \\
\hline 7822.78 & & $8 \mathrm{e}$ & III & & 7655.71 & & 5 & II & 4 & 7507.59 & 5 & & I & \\
\hline 7821.37 & 2 & & I & & 7650.02 & 4 & & I & & 7503.32 & & $1 \mathrm{~h}$ & II & \\
\hline 7820.89 & 50 & & I & & 7648.88 & & 4 & II & 6 & 7501.33 & & $1 \mathrm{~h}$ & II & \\
\hline 7815.52 & & 5 & II & 5 & 7645.84 & & 8 & II & 6 & 7500.09 & & $3 \mathrm{~h}$ & II & \\
\hline 7811.96 & 1 & 8 & II & 4 & 7641.45 & 3 & & I & & 7499.87 & 30 & & I & \\
\hline 7809.48 & & $8 \mathrm{e}$ & III & & 7640.63 & & $1 \mathrm{~h}$ & II & & 7496.33 & 100 & 2 & I & \\
\hline 7808.64 & & 4 & II & 6 & 7639.47 & & 4 & II & 4 & 7496.24 & & 20 & II & \\
\hline 7806.11 & & 3 & II & & 7637.27 & 15 & & I & & 7495.15 & & 2 & II & \\
\hline 7804.57 & & 6 & II & & 7636.65 & & 3 & II & & 7494.37 & & 3 & II & \\
\hline 7803.65 & 20 & 300 & II & 6 & 7635.53 & & $4 \mathrm{~h}$ & III & & 7492.34 & 1 & 6 & II & 4 \\
\hline 7802.01 & & 9 & II & & 7634.62 & & 6 & II & & 7483.19 & & 2 & II & \\
\hline 7801.17 & & 8 & II & & 7633.95 & 6 & 100 & II & 4 & 7482.80 & & 1 & II & \\
\hline 7799.44 & & 3 & II & & 7631.81 & & 4 & II & & 7481.04 & & 6 & II & 7 \\
\hline 7799.33 & 1 & & I & & 7630.87 & & 1 & II & & 7478.77 & 4 & & I & \\
\hline 7793.72 & & $2 \mathrm{~h}$ & III & & 7630.48 & & 9 & II & & 7477.86 & 1 & 5 & II & \\
\hline 7789.80 & 2 & & I & & 7630.00 & & 8 & II & & 7477.61 & 7 & 200 & II & 7 \\
\hline 7785.02 & & 3 & II & 4 & 7627.45 & 1 & 50 & II & 5 & 7475.24 & & 2 & II & \\
\hline 7777.96 & 1 & 7 & II & 6 & 7627.24 & 1 & 70 & II & 7 & 7474.72 & & $4 \mathrm{e}$ & III & \\
\hline 7777.49 & & 6 & II & 5 & 7623.90 & 20 & & I & & 7473.32 & & 5 & II & \\
\hline
\end{tabular}


TABLE 1.-Emission spectra of ytterbium-Continued

\begin{tabular}{|c|c|c|c|c|c|c|c|c|c|c|c|c|c|c|}
\hline \multirow{3}{*}{$\begin{array}{c}\text { Wave- } \\
\text { length } \\
\text { in air }\end{array}$} & \multicolumn{2}{|c|}{ Intensity } & \multirow{3}{*}{ Spectrum } & \multirow{3}{*}{$\begin{array}{c}\text { Zeeman } \\
\text { type }\end{array}$} & \multirow{3}{*}{$\begin{array}{c}\begin{array}{c}\text { Wave- } \\
\text { length } \\
\text { in air }\end{array} \\
\AA \\
\end{array}$} & \multicolumn{2}{|c|}{ Intensity } & \multirow{3}{*}{ Spectrum } & \multirow{3}{*}{$\begin{array}{c}\text { Zeeman } \\
\text { type }\end{array}$} & \multirow{3}{*}{$\begin{array}{c}\text { Wave- } \\
\text { length } \\
\text { in air }\end{array}$} & Inte & nsity & & \\
\hline & Meggers & Thompson & & & & Meggers & Thompson & & & & Meggers & Thompson & Jpectrum & type \\
\hline & lamp & lamp & & & & lamp & lamp & & & & lamp & lamp & & \\
\hline 7472.44 & 2 & & I & & 7342.30 & 10 & 400 & II & 4 & 7191.87 & & 1 & II & \\
\hline 7469.44 & 60 & & I & & 7341.64 & & 4 & II & & 7190.33 & 6 & & I & \\
\hline 7468.09 & & 8 & II & & 7339.99 & 10 & & I & & 7187.07 & 200 & 2 & I & 1 \\
\hline 7467.58 & 40 & 30 & I & & 7339.80 & & 60 & II & 7 & 7182.60 & & 2 & II & \\
\hline 7464.10 & 5 & 60 & II & 4 & 7334.01 & 50 & & I & & 7175.48 & & 300 & II & 6 \\
\hline 7462.71 & & $5 \mathrm{e}$ & III & & 7331.31 & 1 & 80 & II & 6 & 7175.10 & 1000 & 10 & I & 3 \\
\hline 7458.79 & $5 \mathrm{~h}$ & & I & & 7329.29 & & 2 & II & & 7174.60 & & 8 & II & \\
\hline 7456.86 & & $15 \mathrm{e}$ & III & & 7328.57 & 8 & & I & & 7173.96 & 10 & & I & \\
\hline 7456.20 & & 2 & II & & 7327.87 & 500 & 2 & I & 1 & 7169.25 & 10 & & I & \\
\hline 7451.21 & & 1 & II & & 7325.07 & & 2 & II & & 7160.31 & 2 & & I & \\
\hline 7449.60 & & 1 & II & & 7323.32 & 4 & & I & & 7157.72 & & $15 \mathrm{e}$ & III & \\
\hline 7448.28 & 6000 & 100 & I & 7 & 7318.77 & & 1 & II & & 7156.48 & 3 & & I & \\
\hline 7447.37 & 1 & 60 & II & 7 & 7317.74 & & 30 & II & 7 & 7154.52 & & 5 & II & 6 \\
\hline 7444.20 & & 2 & II & & 7313.18 & & 15 & II & & 7153.51 & 3 & & I & \\
\hline 7439.84 & 1 & 80 & II & 5 & 7313.05 & 3000 & 50 & I & 2 & 7152.40 & 10 & & I & \\
\hline 7438.92 & 25 & 1 & I & & 7311.02 & & $10 \mathrm{e}$ & III & & 7148.16 & & 2 & II & \\
\hline 7433.39 & 4 & 30 & II & 5 & 7310.70 & 400 & 1 & I & & 7147.94 & & 8 & II & \\
\hline 7429.51 & 2 & 40 & II & 4 & 7309.19 & & 40 & II & 5 & 7145.13 & & 4 & II & 7 \\
\hline 7429.10 & & 2 & II & & 7306.63 & 1 & 100 & II & 5 & 7142.39 & 1 & 120 & II & 5 \\
\hline 7422.15 & 40 & 200 & II & 5 & 7305.92 & & 8 & II & & 7141.28 & 6 & & I & \\
\hline 7421.04 & & 2 & II & & 7305.22 & 2500 & 50 & I & 3 & 7137.67 & & 2 & II & \\
\hline 7419.60 & 1 & 40 & II & 4 & 7304.54 & & $5 \mathrm{e}$ & III & & 7136.82 & 5 & & I & \\
\hline 7419.22 & 10 & & I & & 7302.15 & & 15 & II & 4 & 7135.61 & 10 & & I & \\
\hline 7419.06 & & 8 & II & & 7298.05 & $8 \mathrm{~h}$ & & I & & 7134.85 & 5 & & I & \\
\hline 7418.49 & & 4 & II & & 7294.50 & & 1 & II & & 7134.83 & & 2 & II & \\
\hline 7418.15 & & 3 & II & & 7290.56 & & 3 & II & & 7128.94 & & 2 & II & \\
\hline 7416.64 & 40 & & I & & 7287.96 & & 2 & II & & 7126.81 & 2 & 100 & II & 5 \\
\hline 7414.13 & 5 & & I & & 7285.02 & & 1 & II & & 7124.46 & & 5 & II & 5 \\
\hline 7411.63 & & 4 & II & 5 & 7284.70 & & 1 & II & & 7117.99 & & 10 & II & 6 \\
\hline 7411.17 & 200 & 2 & I & & 7279.67 & & 5 & II & & 7116.96 & & 3 & III & \\
\hline 7410.94 & & $8 \mathrm{e}$ & III & & 7278.37 & & 30 & II & 4 & 7108.98 & 200 & 2 & I & \\
\hline 7410.01 & & $80 \mathrm{e}$ & III & & 7275.02 & & $1 \mathrm{e}$ & III & & 7108.37 & & 6 & II & 6 \\
\hline 7406.00 & 10 & 150 & II & 6 & 7268.03 & & 4 & II & & 7105.34 & & 1 & III & \\
\hline 7405.93 & 6 & & I & & 7266.55 & & $1 \mathrm{e}$ & III & & 7101.66 & & 10 & II & 4 \\
\hline 7404.35 & 10 & & I & & 7266.37 & & $1 \mathrm{e}$ & III & & 7099.70 & & 2 & II & \\
\hline 7402.70 & & 2 & II & & 7265.76 & & 10 & II & 7 & 7098.11 & & 6 & II & 4 \\
\hline 7402.45 & & 1 & II & & 7259.76 & & 6 & II & & 7089.40 & & 1 & II & \\
\hline 7400.97 & & 5 & II & & 7259.56 & & 3 & II & & 7086.08 & & 20 & II & 4 \\
\hline 7400.67 & 7 & & I & & 7256.62 & 20 & & I & & 7082.61 & & 80 & II & 4 \\
\hline 7399.98 & & $10 \mathrm{e}$ & III & & 7253.76 & 40 & & I & & 7081.81 & & 4 & II & \\
\hline 7398.50 & & $3 \mathrm{e}$ & III & & 7251.55 & 30 & 200 & II & 6 & 7080.77 & & 1 & II & \\
\hline 7397.10 & 1 & 40 & II & 4 & 7244.41 & 2000 & 40 & I & 3 & 7080.53 & & 40 & II & 6 \\
\hline 7396.04 & & 1 & II & & 7238.95 & 1 & 80 & II & & 7078.11 & & 5 & II & 7 \\
\hline 7393.34 & 4 & 30 & II & 4 & 7238.73 & 4 & 80 & II & & 7077.07 & & 1 & II & \\
\hline 7393.03 & & 1 & II & & 7235.42 & 1 & 40 & II & 6 & 7074.60 & & 2 & II & \\
\hline 7389.22 & 6 & 150 & II & 5 & 7234.00 & & $3 \mathrm{e}$ & III & & 7072.12 & & 4 & II & 4 \\
\hline 7387.69 & 2 & & I & & 7229.70 & & $3 \mathrm{e}$ & III & & 7066.20 & & 10 & II & \\
\hline 7384.50 & 7 & 150 & II & 7 & 7228.98 & & 2 & II & 5 & 7060.67 & & 60 & II & 4 \\
\hline 7383.00 & & 2 & II & & 7227.21 & 5 & & I & & 7060.41 & & 2 & II & \\
\hline 7382.17 & 3 & & I & & 7222.72 & 20 & 150 & II & 6 & 7056.72 & & 4 & II & 5 \\
\hline 7377.73 & 20 & 400 & II & & 7221.50 & & 10 & II & & 7053.80 & 1 & 200 & II & 7 \\
\hline 7377.43 & 10 & 300 & II & & 7221.21 & 1 & 60 & II & 7 & 7052.58 & & 10 & II & 5 \\
\hline 7373.06 & 3 & 15 & II & 4 & 7220.38 & 2 & 50 & II & 5 & 7045.75 & & 15 & II & \\
\hline 7367.93 & & $8 \mathrm{e}$ & III & & 7217.57 & & 60 & II & 5 & 7045.51 & & 20 & II & \\
\hline 7367.78 & & 20 & II & 4 & 7209.65 & & 1 & II & & 7043.78 & 30 & 700 & II & 7 \\
\hline 7362.92 & 2 & 15 & II & 5 & 7209.38 & & 2 & II & & 7037.04 & & $10 \mathrm{e}$ & III & \\
\hline 7362.83 & & 60 & II & & 7209.10 & & 8 & II & & 7032.91 & 2 & & I & \\
\hline 7361.90 & 200 & 1 & I & & 7208.29 & 4 & 200 & II & 7 & 7031.52 & & 30 & II & 6 \\
\hline 7356.90 & 3 & & I & & 7205.93 & 4 & & I & & 7031.21 & 3 & & I & \\
\hline 7354.17 & & 4 & II & 6 & 7202.20 & 100 & 1 & I & 3 & 7029.38 & & 3 & II & \\
\hline 7350.42 & & 15 & II & & 7201.09 & & 20 & II & 7 & 7029.27 & & 2 & II & \\
\hline 7350.04 & 6000 & 100 & I & 3 & 7198.08 & $10 \mathrm{~h}$ & & I & & 7027.84 & 10 & 150 & II & \\
\hline 7346.60 & 3 & & I & & 7196.92 & 3 & & I & & 7023.97 & & 6 & II & \\
\hline
\end{tabular}


TABLE 1.-Emission spectra of ytterbium-Continued

\begin{tabular}{|c|c|c|c|c|c|c|c|c|c|c|c|c|c|c|}
\hline \multirow{3}{*}{$\begin{array}{c}\text { Wave- } \\
\text { length } \\
\text { in air }\end{array}$} & \multicolumn{2}{|c|}{ Intensity } & \multirow{3}{*}{ Spectrum } & \multirow{3}{*}{$\begin{array}{c}\text { Zeeman } \\
\text { type }\end{array}$} & \multirow{3}{*}{$\begin{array}{c}\text { Wave- } \\
\text { length } \\
\text { in air }\end{array}$} & \multicolumn{2}{|c|}{ Intensity } & \multirow{3}{*}{ Spectrum } & \multirow{3}{*}{$\begin{array}{c}\text { Zeeman } \\
\text { type }\end{array}$} & \multirow{3}{*}{$\begin{array}{c}\text { Wave- } \\
\text { length } \\
\text { in air }\end{array}$} & & ensity & & \\
\hline & Meggers & Thompson & & & & Meggers & Thompson & & & & Meggers & Thompson & | & \\
\hline & lamp & lamp & & & & $\operatorname{lamp}$ & lamp & & & & lamp & lamp & & \\
\hline 7022.27 & & 6 & II & & 6862.08 & $2 \mathrm{~h}$ & & I & & 6728.37 & & 1 & II & \\
\hline 7022.00 & 2 & 200 & II & 4 & 6857.64 & & 2 & II & & 6727.61 & 400 & 2000 & II & 7 \\
\hline 7020.18 & 500 & 4 & I & & 6854.67 & & $7 \mathrm{e}$ & III & & 6727.12 & & 20 & II & \\
\hline 7019.48 & 2 & 20 & II & 5 & 6850.64 & 4 & & I & & 6724.92 & 1 & & I & \\
\hline 7012.75 & & 5 & II & 6 & 6844.05 & & 1 & II & & 6724.36 & 1 & 30 & II & 6 \\
\hline 7003.90 & & 1 & II & & 6843.02 & & 2 & II & & 6724.19 & & 1 & II & \\
\hline 7002.14 & & 1 & II & & 6842.87 & & 3 & III & & 6723.90 & & 1 & II & \\
\hline 7001.79 & 3 & & I & & 6840.56 & & 3 & III & & 6720.85 & 2 & & I & \\
\hline 6999.88 & 50 & 800 & II & 6 & 6833.56 & 4 & & I & & 6719.87 & $6 \mathrm{~h}$ & & I & \\
\hline 6998.98 & & 2 & II & & 6831.17 & 7 & & I & & 6718.00 & 1 & 30 & II & \\
\hline 6993.31 & 5 & 250 & II & 4 & 6830.99 & & 2 & II & & 6717.05 & 30 & & I & \\
\hline 6990.51 & 100 & & I & & 6829.80 & 1 & 70 & II & & 6715.79 & 1000 & 20 & I & 7 \\
\hline 6985.15 & & $20 \mathrm{e}$ & III & & 6828.90 & 2 & 200 & II & 4 & 6710.95 & & 1 & II & \\
\hline 6981.99 & 20 & 300 & II & 4 & 6828.35 & & 2 & II & & 6710.73 & & 3 & II & \\
\hline 6978.36 & & 5 & II & & 6826.18 & & 5 & II & & 6707.91 & 3 & & I & \\
\hline 6977.67 & 20 & 150 & II & 6 & 6826.02 & & 10 & II & & 6707.76 & 5 & & I & \\
\hline 6977.30 & & 1 & II & & 6819.41 & & 2 & II & & 6707.61 & & 15 & II & \\
\hline 6976.89 & $3 \mathrm{~h}$ & & I & & 6817.16 & 100 & & 1 & & 6702.48 & 2 & & I & \\
\hline 6973.58 & 10 & & I & & 6816.18 & & 40 & II & & 6701.26 & 15 & & I & \\
\hline 6970.54 & & 7 & II & & 6815.88 & 1 & 100 & II & 6 & 6699.36 & 100 & 1000 & II & 4 \\
\hline 6968.73 & 1 & 30 & II & 6 & 6813.65 & 5 & & I & & 6698.93 & & 5 & II & \\
\hline 6965.46 & 1 & & I & & 6812.42 & & 2 & II & & 6698.13 & & 3 & II & \\
\hline 6964.47 & & 3 & II & & 6809.30 & & 5 & II & 4 & 6697.64 & $10 \mathrm{~h}$ & & I & \\
\hline 6963.47 & & 10 & II & & 6806.67 & 10 & & I & & 6692.42 & 700 & 5 & I & 1 \\
\hline 6963.10 & 5 & 300 & II & 6 & 6803.91 & & 20 & II & & 6689.84 & & 15 & II & \\
\hline 6959.11 & 1 & 10 & II & 5 & 6802.47 & 2 & 400 & II & 5 & 6689.28 & 10 & 150 & II & 5 \\
\hline 6958.11 & 300 & 3 & I & & 6799.60 & $60000 \mathrm{R}$ & 6000 & I & 3 & 6687.82 & 60 & & I & \\
\hline 6953.29 & 1 & & I & & 6798.29 & & & II & & 6687.64 & & 1 & II & \\
\hline 6952.14 & & 4 & II & & 6796.80 & & $2 \mathrm{~h}$ & II & & 6685.34 & & 1 & II & \\
\hline 6951.38 & 50 & & I & & 6792.58 & & 30 & II & & 6683.41 & & 10 & II & \\
\hline 6949.50 & & 10 & II & 4 & 6790.82 & & 20 & II & 6 & 6682.06 & & 15 & II & \\
\hline 6944.95 & & 30 & II & 6 & 6786.32 & & 1 & II & & 6680.74 & & 4 & II & \\
\hline 6943.98 & 4 & & I & & 6785.14 & 1 & 150 & II & 4 & 6679.30 & 1 & 100 & II & \\
\hline 6934.05 & 100 & 600 & II & 6 & 6784.80 & & $4 \mathrm{e}$ & III & & 6678.17 & 20000 & 1000 & I & 3 \\
\hline 6929.57 & & 9 & III & & 6782.17 & 1000 & 20 & I & 3 & 6673.08 & & 10 & II & 4 \\
\hline 6926.08 & 1 & 30 & II & 5 & 6779.74 & 2 & & I & & 6670.57 & & 1 & II & \\
\hline 6923.78 & & 2 & II & & 6777.22 & 600 & 10 & I & & 6670.18 & & 50 & II & \\
\hline 6921.30 & & 5 & II & 5 & 6776.87 & & $2 \mathrm{e}$ & III & & 6667.82 & 50000 & 2000 & I & 7 \\
\hline 6916.78 & & 1 & II & & 6775.79 & & 2 & II & & 6666.78 & & 20 & II & \\
\hline 6916.10 & & 3 & II & & 6773.47 & 2 & & I & & 6666.55 & & 40 & II & 4 \\
\hline 6915.84 & & 4 & II & 7 & 6772.42 & 1 & 50 & II & 6 & 6665.08 & & 1 & II & \\
\hline 6914.84 & 2 & & I & & 6772.26 & 20 & & I & & 6661.90 & 2 & 200 & II & 6 \\
\hline 6913.73 & 50 & 1 & I & & 6768.70 & 6000 & 1000 & I & 2 & 6658.43 & & 60 & II & 6 \\
\hline 6911.34 & & 1 & II & & 6765.67 & 40 & 1 & I & & 6657.26 & & 25 & II & \\
\hline 6911.11 & 1 & 20 & II & 5 & 6765.24 & 200 & 1 & I & 7 & 6656.70 & 2 & & I & \\
\hline 6908.92 & & 2 & II & & 6761.64 & $3 \mathrm{~h}$ & & I & & 6651.52 & & 4 & II & \\
\hline 6902.28 & 2 & & I & & 6761.31 & & 4 & II & 4 & 6647.25 & & 1 & II & \\
\hline 6901.06 & & 2 & II & & 6759.10 & & 15 & II & 4 & 6644.94 & & 6 & II & \\
\hline 6897.78 & & 1 & II & & 6757.29 & 1 & & I & & 6644.06 & 2 & 200 & II & 4 \\
\hline 6897.31 & 1 & 40 & II & 5 & 6755.44 & 1 & 150 & II & 6 & 6643.55 & 10000 & 300 & I & 7 \\
\hline 6892.61 & 5 & & I & & 6754.69 & & 3 & II & & 6642.12 & $10 \mathrm{~h}$ & & I & \\
\hline 6891.94 & 20 & & I & & 6753.01 & 2 & & I & & 6640.88 & & 15 & II & \\
\hline 6890.19 & & 3 & III & & 6751.45 & & 10 & II & & 6640.79 & 7 & & I & \\
\hline 6889.59 & 10 & 100 & II & 5 & 6751.17 & & $2 \mathrm{e}$ & III & & 6639.62 & & 3 & II & \\
\hline 6888.76 & & 2 & II & & 6749.40 & 1000 & 20 & I & 2 & 6639.34 & 150 & 1 & 1 & \\
\hline 6886.95 & & 8 & II & 4 & 6746.64 & & 6 & II & & 6639.19 & & 5 & II & \\
\hline 6886.77 & & 5 & II & & 6745.23 & 30 & 600 & II & 7 & 6636.54 & & 2 & II & \\
\hline 6881.40 & 1 & 100 & II & 5 & 6740.59 & & 8 & II & & 6636.42 & $6 \mathrm{~h}$ & & I & \\
\hline 6877.93 & 2 & 250 & II & 7 & 6738.24 & & 2 & II & & 6634.28 & 5 & & I & \\
\hline 6871.51 & & 3 & II & & 6734.56 & & 1 & II & & 6632.40 & & 2 & II & \\
\hline 6869.56 & 200 & 1 & I & & 6732.53 & & 15 & II & 7 & 6630.85 & $2 \mathrm{~h}$ & & I & \\
\hline 6867.95 & & 2 & III & & 6732.17 & 2 & & I & & 6626.73 & 800 & 2 & I & 2 \\
\hline 6864.27 & 2 & 300 & & 6 & 6731.02 & $5 \mathrm{~h}$ & & I & & 6626.39 & & $2 \mathrm{e}$ & III & \\
\hline
\end{tabular}


TABLE 1.-Emission spectra of ytterbium-Continued

\begin{tabular}{|c|c|c|c|c|c|c|c|c|c|c|c|c|c|c|}
\hline \multirow{3}{*}{$\begin{array}{c}\text { Wave- } \\
\text { length } \\
\text { in air }\end{array}$} & \multicolumn{2}{|c|}{ Intensity } & \multirow{3}{*}{ Spectrum } & \multirow{3}{*}{$\begin{array}{c}\text { Zeeman } \\
\text { type }\end{array}$} & \multirow{3}{*}{$\begin{array}{c}\text { Wave- } \\
\text { length } \\
\text { in air }\end{array}$} & \multicolumn{2}{|c|}{ Intensity } & \multirow{3}{*}{ Spectrum } & \multirow{3}{*}{$\begin{array}{c}\text { Zeeman } \\
\text { type }\end{array}$} & \multirow{3}{*}{$\begin{array}{c}\text { Wave- } \\
\text { length } \\
\text { in air }\end{array}$} & Inter & nsity & & \\
\hline & Meggers & Thompson & & & & Meggers & Thompson & & & & Meggers & Thompson & spectrum & type \\
\hline & lamp & lamp & & & & lamp & lamp & & & & lamp & lamp & & \\
\hline 6625.27 & $4 \mathrm{~h}$ & & I & & 6512.23 & & $1 \mathrm{e}$ & III & & 6417.91 & 20000 & 1000 & I & 2 \\
\hline 6619.66 & & 40 & II & & 6509.09 & 60 & 1 & I & & 6416.98 & & 7 & II & \\
\hline 6617.72 & & 4 & II & & 6508.81 & 1 & & I & & 6412.85 & & 30 & II & \\
\hline 6617.06 & 100 & 1000 & II & 6 & 6507.98 & 2 & & I & & 6411.38 & & $7 \mathrm{e}$ & III & \\
\hline 6616.40 & 2 & 40 & II & & 6507.15 & & 1 & II & & 6408.77 & 4 & & I & \\
\hline 6615.80 & 1 & 80 & II & & 6505.29 & 1 & & I & & 6408.44 & 4 & & I & \\
\hline 6611.87 & & 4 & II & & 6503.96 & 3 & & I & & 6404.62 & 800 & 20 & I & \\
\hline 6611.32 & & 5 & II & & 6503.01 & 50 & 500 & II & 4 & 6402.64 & & 50 & II & \\
\hline 6610.79 & & $1 \mathrm{e}$ & III & & 6502.28 & & 20 & II & & 6400.35 & 40000 & 2000 & I & 7 \\
\hline 6610.31 & & 1 & II & & 6500.70 & 2 & 30 & II & & 6397.15 & & 2 & II & \\
\hline 6608.68 & & 1 & II & & 6498.79 & 1 & 20 & II & & 6396.68 & & 1 & II & \\
\hline 6607.07 & 2000 & 40 & I & 1 & 6493.58 & & 5 & II & & 6396.09 & & 7 & II & \\
\hline 6605.82 & 4 & 250 & II & 7 & 6492.73 & 10 & 700 & II & 4 & 6393.91 & & 3 & II & \\
\hline 6604.45 & & 50 & II & & 6491.18 & & 5 & II & & 6393.74 & 700 & 5 & I & 2 \\
\hline 6600.68 & & 7 & II & & 6490.32 & & 20 & II & & 6393.59 & & 8 & II & \\
\hline 6600.03 & & 2 & II & & 6489.06 & $80000 \mathrm{R}$ & 10000 & I & 2 & 6393.23 & 4 & 150 & II & 4 \\
\hline 6594.65 & $3 \mathrm{~h}$ & & I & & 6488.47 & & 200 & II & & 6389.69 & & 10 & II & \\
\hline 6593.25 & & 50 & II & & 6487.08 & & 10 & II & & 6387.68 & 100 & 2 & I & \\
\hline 6592.69 & 2 & 150 & II & 7 & 6486.74 & & 50 & II & & 6386.84 & & 25 & II & \\
\hline 6592.47 & 40 & & I & & 6485.55 & 10 & $200 \mathrm{~h}$ & II & & 6382.92 & 20 & 200 & II & 7 \\
\hline 6592.12 & & 30 & II & & 6477.98 & 5 & & I & & 6381.68 & 10 & & I & \\
\hline 6590.72 & 1 & 15 & II & & 6477.63 & & 6 & II & & 6380.47 & & 3 & II & \\
\hline 6587.26 & & 1 & II & & 6476.36 & & 1 & II & & 6378.33 & & $150 \mathrm{e}$ & III & \\
\hline 6585.41 & 100 & 1000 & II & 6 & 6475.98 & 8 & & I & & 6377.01 & 6 & 400 & II & 7 \\
\hline 6582.79 & 2 & 250 & II & 7 & 6474.74 & 30 & 1500 & II & 6 & 6376.74 & 1 & 20 & II & \\
\hline 6578.10 & & 20 & II & & 6470.17 & & 1 & II & & 6374.81 & 4 & 80 & II & 5 \\
\hline 6574.10 & 2 & 200 & II & 6 & 6468.88 & 70 & & I & & 6372.72 & 1500 & 20 & I & 1 \\
\hline 6573.31 & & 10 & II & & 6468.18 & 1500 & 5 & I & & 6371.34 & & $2 \mathrm{e}$ & III & \\
\hline 6572.90 & 300 & 2 & I & & 6466.79 & & 7 & II & & 6367.91 & & 4 & II & \\
\hline 6571.44 & 10 & 400 & II & 4 & 6466.33 & & $25 \mathrm{e}$ & III & & 6365.88 & & $10 \mathrm{e}$ & III & \\
\hline 6570.94 & & 9 & II & & 6465.78 & & $3 \mathrm{~h}$ & II & & 6365.05 & 30 & & I & \\
\hline 6568.35 & 60 & 1 & 1 & & 6464.66 & 3 & & I & & 6364.92 & & 3 & II & \\
\hline 6567.60 & & 9 & II & & 6464.46 & 1 & & I & & 6360.33 & & 4 & II & \\
\hline 6565.72 & 1 & & I & & 6463.15 & 50 & 2000 & II & 7 & 6359.22 & $2 \mathrm{~h}$ & & I & \\
\hline 6563.38 & & 2 & II & & 6461.37 & $3 \mathrm{~h}$ & & I & & 6358.49 & 20 & & I & \\
\hline 6562.35 & & $5 \mathrm{~h}$ & II & & 6460.89 & 2 & & I & & 6356.67 & 2 & 200 & II & 5 \\
\hline 6561.65 & 3 & & 1 & & 6460.66 & 1 & & I & & 6355.38 & 20 & 1000 & II & 4 \\
\hline 6560.94 & & $1 \mathrm{~h}$ & II & & 6456.95 & 2 & 150 & II & & 6354.39 & & 20 & II & \\
\hline 6559.65 & & 2 & II & & 6456.90 & 2 & & I & & 6348.85 & $2 \mathrm{~h}$ & & I & \\
\hline 6557.46 & & 4 & II & & 6456.64 & 1 & & I & & 6348.05 & & 10 & II & \\
\hline 6555.15 & 1500 & 15 & I & 7 & 6456.33 & 5 & & I & & 6347.32 & & 20 & II & \\
\hline 6553.35 & 900 & 9 & I & 3 & 6455.41 & 1000 & & I & & 6347.08 & & $4 \mathrm{e}$ & III & \\
\hline 6552.61 & & 2 & II & & 6455.23 & & 4 & II & & 6345.74 & 40 & 400 & II & \\
\hline 6550.90 & 1 & & I & & 6453.30 & 1 & 150 & II & & 6344.97 & 3000 & 100 & I & 3 \\
\hline 6550.14 & 400 & 10 & I & 3 & 6453.11 & 10 & 150 & II & & 6343.54 & & 7 & II & \\
\hline 6549.16 & & $6 \mathrm{e}$ & III & & 6452.73 & 1 & & I & & 6343.01 & 8 & & I & \\
\hline 6548.53 & 2 & 200 & II & 6 & 6452.46 & 1 & & I & & 6342.65 & & 1 & II & \\
\hline 654.7 .82 & $4 \mathrm{~h}$ & & I & & 6452.10 & & 15 & II & & 6341.41 & & 6 & II & \\
\hline 6547.77 & & 9 & II & & 6450.88 & 40 & & I & & 6340.93 & & 15 & II & 7 \\
\hline 6547.43 & 2 & & I & & 6450.10 & & $4 \mathrm{e}$ & III & & 6338.10 & & 7 & II & \\
\hline 6546.35 & & 2 & II & & 6449.59 & 1500 & 15 & I & & 6336.23 & 100 & 1 & I & \\
\hline 6541.38 & 3 & 400 & II & 6 & 6448.41 & $4 \mathrm{~h}$ & & I & & 6335.72 & 3000 & 70 & I & 1 \\
\hline 6540.36 & & 1 & II & & 6446.22 & 20 & & I & & 6334.20 & & 5 & II & \\
\hline 6539.33 & & $3 \mathrm{e}$ & III & & 6442.10 & & 9 & II & & 6333.45 & 1 & 70 & II & \\
\hline 6538.31 & 20 & & I & & 6440.79 & 5 & 400 & II & 6 & 6328.52 & & $200 \mathrm{e}$ & III & \\
\hline 6531.71 & & 5 & II & & 6437.98 & 1 & 150 & II & & 6328.19 & 4 & & I & \\
\hline 6531.26 & & 4 & II & & 6435.61 & $3 \mathrm{~h}$ & & I & & 6324.77 & 40 & 400 & II & 5 \\
\hline 6531.00 & 25 & 1 & I & & 6433.44 & 15 & 1 & I & & 6324.43 & 1 & 90 & II & 4 \\
\hline 6527.01 & & 25 & II & & 6432.73 & 1000 & 3000 & II & 5 & 6322.56 & 400 & 3 & I & 2 \\
\hline 6526.36 & & 3 & II & & 6427.60 & 20 & 400 & II & 5 & 6318.91 & 20 & 1 & I & \\
\hline 6523.19 & 2 & 250 & II & & 6423.67 & & 5 & II & & 6317.78 & & $2 \mathrm{e}$ & III & \\
\hline 6521.51 & & 25 & II & & 6421.53 & 1500 & 10 & I & 2 & 6313.61 & & 1 & II & \\
\hline 6513.78 & 5 & & I & & 6421.04 & 400 & 20 & I & & 6312.99 & & 20 & II & 6 \\
\hline
\end{tabular}


TABLE 1.-Emission spectra of ytterbium-Continued

\begin{tabular}{|c|c|c|c|c|c|c|c|c|c|c|c|c|c|c|}
\hline \multirow{3}{*}{$\begin{array}{c}\text { Wave- } \\
\text { length } \\
\text { in air }\end{array}$} & \multicolumn{2}{|c|}{ Intensity } & \multirow{3}{*}{ Spectrum } & \multirow{3}{*}{$\begin{array}{c}\text { Zeeman } \\
\text { type }\end{array}$} & \multirow{3}{*}{$\begin{array}{c}\text { Wave- } \\
\text { length } \\
\text { in air }\end{array}$} & \multicolumn{2}{|c|}{ Intensity } & \multirow{3}{*}{ Spectrum } & \multirow{3}{*}{$\begin{array}{c}\text { Zeeman } \\
\text { type }\end{array}$} & \multirow{3}{*}{$\begin{array}{c}\text { Wave- } \\
\text { length } \\
\text { in air }\end{array}$} & Inte & nsity & & \\
\hline & Meggers & Thompson & & & & Meggers & Thompson & & & & Meggers & Thompson & spectrom & type \\
\hline & lamp & lamp & & & & lamp & lamp & & & & lamp & lamp & & \\
\hline 6311.10 & 2 & & 1 & & 6209.05 & 2 & & I & & 6121.91 & & 9 & II & \\
\hline 6309.82 & 20 & 1 & 1 & & 6208.89 & 3 & 70 & II & & 6120.30 & 5 & 250 & II & \\
\hline 6308.15 & 400 & 2000 & II & 5 & 6208.10 & 10 & 500 & II & 6 & 6118.28 & 3000 & 100 & I & 7 \\
\hline 6305.37 & 90 & 1 & I & & 6206.49 & & 3 & II & & 6117.54 & 2 & 10 & II & \\
\hline 6304.79 & 8 & & I & & 6204.88 & & 4 & II & & 6116.67 & 10 & 70 & II & \\
\hline 6303.91 & & 5 & II & & 6204.20 & & 4 & II & & 6113.42 & 10 & & I & \\
\hline 6303.27 & 50 & 400 & II & 5 & 6198.67 & 20 & & I & & 6111.27 & 4000 & 60 & I & 2 \\
\hline 6300.94 & & 8 & II & & 6196.90 & 3 & & I & & 6110.14 & 6 & 200 & II & 7 \\
\hline 6299.71 & 2 & & I & & 6196.01 & & 6 & II & & 6108.32 & & 2 & II & \\
\hline 6297.95 & 90 & 1 & I & & 6194.85 & 900 & 4 & I & 2 & 6106.18 & 5 & 200 & II & 6 \\
\hline 6297.35 & 5 & 400 & II & & 6192.32 & 60 & 1 & I & & 6105.58 & 1 & 10 & II & \\
\hline 6292.56 & & 2 & II & & 6191.14 & & $7 \mathrm{~h}$ & II & & 6104.79 & & $2 \mathrm{e}$ & III & \\
\hline 6292.17 & 2 & 20 & II & & 6190.78 & 15 & 1000 & II & 7 & 6101.83 & 40 & & I & \\
\hline 6291.06 & & 5 & II & & 6189.03 & 1 & 90 & II & & 6099.35 & & 3 e & III & \\
\hline 6286.25 & 2000 & 20 & I & 2 & 6187.08 & & 20 & II & & 6098.89 & & 7 & II & \\
\hline 6285.72 & & 3 & II & & 6185.43 & & 2 & II & & 6098.54 & 3 & 250 & II & \\
\hline 6283.00 & & $1 \mathrm{e}$ & III & & 6184.05 & & 1 & II & & 6096.98 & 1 & 30 & II & \\
\hline 6281.96 & & 4 & II & & 6183.01 & 2 & & I & & 6096.15 & 15 & 2 & I & \\
\hline 6280.60 & & 2 & II & & 6182.14 & 1 & & I & & 6095.98 & & 3 & II & \\
\hline 6280.39 & & $5 \mathrm{e}$ & III & & 6181.78 & 100 & 2 & I & 7 & 6094.49 & 4 & & I & \\
\hline 6279.21 & & $1 \mathrm{e}$ & III & & 6178.81 & 1 & 20 & II & & 6089.14 & 20 & 100 & II & \\
\hline 6277.08 & 2 & 150 & II & 7 & 6178.63 & & 3 & II & & 6088.70 & 3 & 200 & II & 7 \\
\hline 6274.78 & 2000 & 10000 & II & 6 & 6176.91 & 1 & & I & & 6087.44 & 20 & & I & \\
\hline 6271.15 & 6 & 250 & II & 4 & 6175.62 & 150 & 50 & I & & 6086.48 & 2 & & I & \\
\hline 6270.31 & 3 & 100 & II & 5 & 6175.58 & & 90 & II & 4 & 6083.86 & 1 & 30 & II & \\
\hline 6269.95 & 1 & 40 & II & & 6175.36 & & 9 & II & & 6083.29 & 5 & 200 & II & 5 \\
\hline 6265.45 & 1 & 80 & II & 7 & 6174.70 & 1 & 60 & II & & 6082.37 & 6 & 500 & II & 6 \\
\hline 6262.89 & & 15 & II & & 6173.76 & & 7 & II & & 6082.21 & & 10 & II & \\
\hline 6262.43 & 1 & & I & & 6172.53 & 5 & & I & & 6076.55 & & $2 \mathrm{e}$ & III & \\
\hline 6261.50 & 2 & & I & & 6171.87 & 20 & & 1 & & 6075.21 & 3 & 200 & II & 7 \\
\hline 6260.79 & 30 & 1500 & II & 7 & 6171.63 & 30 & 300 & II & 6 & 6074.10 & & 1 & II & \\
\hline 6257.03 & 20 & & I & & 6169.55 & 100 & 200 & II & & 6073.17 & 7 & & I & \\
\hline 6255.43 & 200 & 2 & I & & 6164.53 & & 2 & II & & 6070.19 & & 20 & II & \\
\hline 6251.31 & & $2 \mathrm{e}$ & III & & 6161.97 & 50 & & I & & 6068.64 & 1 & 60 & II & \\
\hline 6250.39 & & 1 & II & & 6160.74 & 6 & & I & & 6066.51 & 15 & & I & \\
\hline 6249.54 & & 1 & II & & 6156.03 & 2 & & 1 & & 6065.72 & 300 & 3 & 1 & 2 \\
\hline 6248.29 & & 20 & II & & 6152.57 & 800 & 4000 & II & 6 & 6063.01 & & 4 & II & \\
\hline 6247.99 & 1500 & 15 & I & 3 & 6150.63 & & $70 \mathrm{~h}$ & II & & 6059.93 & & 3 & II & \\
\hline 6246.97 & 500 & 4000 & II & 4 & 6149.44 & & 1 & II & & 6059.22 & 6000 & 80 & I & \\
\hline 6245.93 & & 6 & II & & 6148.50 & 15 & & I & & 6056.46 & 10 & 500 & II & 4 \\
\hline 6244.69 & 3 & & I & & 6148.24 & 1 & & I & & 6055.85 & & $40 \mathrm{e}$ & III & \\
\hline 6244.39 & & 1 & II & & 6146.91 & 10 & 400 & II & 7 & 6054.57 & 4000 & 50 & I & 7 \\
\hline 6242.25 & 1 & 30 & II & & 6146.29 & & 3 & II & & 6053.61 & & 3 & II & \\
\hline 6239.67 & & 3 & II & & 6144.20 & $3 \mathrm{~h}$ & & I & & 6052.88 & 100 & 900 & II & 5 \\
\hline 6236.82 & & $5 \mathrm{e}$ & III & & 6142.83 & 1 & 60 & II & 7 & 6052.66 & & 250 & II & \\
\hline 6236.55 & 600 & 5 & I & & 6141.70 & & 1 & II & & 6052.63 & 150 & & I & \\
\hline 6236.13 & 3 & 150 & II & 4 & 6141.05 & & 3 & II & & 6050.48 & 2 & & I & \\
\hline 6235.25 & 200 & 3 & I & & 6140.72 & & $5 \mathrm{e}$ & III & & 6048.87 & & 2 & II & \\
\hline 6234.85 & 3 & 250 & II & 6 & 6140.56 & & 2 & II & & 6048.44 & 4000 & 50 & I & 1 \\
\hline 6234.12 & 25 & & I & & 6135.42 & & 2 & II & & 6048.18 & 5 & 50 & II & \\
\hline 6233.37 & 5 & 250 & II & 4 & 6134.28 & 7 & 200 & II & 4 & 6046.91 & 9 & & I & \\
\hline 6232.02 & & 2 & II & & 6133.56 & & 3 & II & & 6045.95 & $6 \mathrm{~h}$ & & I & \\
\hline 6229.36 & & 10 & II & & 6132.95 & & 25 & II & & 6045.52 & & 6 & II & \\
\hline 6228.53 & 1 & & I & & 6131.53 & 1 & 80 & II & 5 & 6044.96 & $3 \mathrm{~h}$ & & I & \\
\hline 6223.63 & 15 & 500 & II & 7 & 6130.67 & 1 & & I & & 6043.45 & & 1 & II & \\
\hline 6219.16 & 15 & & I & & 6128.51 & & 5 & II & & 6042.22 & 2 & 100 & II & \\
\hline 6216.14 & & 9 & II & & 6128.18 & 10 & 300 & II & & 6041.79 & 100 & 1 & I & \\
\hline 6215.56 & 6 & 200 & II & 6 & 6127.92 & & 10 & II & & 6040.77 & 15 & 500 & II & 6 \\
\hline 6214.22 & & $100 \mathrm{e}$ & III & & 6126.89 & & 1 & II & & 6038.82 & & 9 & II & \\
\hline 6213.74 & & 3 & II & & 6126.39 & 3 & 100 & II & & 6035.72 & 400 & 4 & I & 7 \\
\hline 6212.28 & & 2 & II & & 6124.63 & & 3 & II & & 6035.37 & 3 & & I & \\
\hline 6211.10 & & 1 & II & & 6123.68 & 1 & 30 & II & & 6034.26 & 1 & & I & \\
\hline & 900 & 1 & & & 6122.99 & 40 & 250 & II & 6 & 6031.80 & 3000 & 40 & I & 3 \\
\hline
\end{tabular}


TABLE 1.-Emission spectra of ytterbium-Continued

\begin{tabular}{|c|c|c|c|c|c|c|c|c|c|c|c|c|c|c|}
\hline \multirow{3}{*}{$\begin{array}{c}\text { Wave- } \\
\text { length } \\
\text { in air }\end{array}$} & \multicolumn{2}{|c|}{ Intensity } & \multirow{3}{*}{ Spectrum } & \multirow{3}{*}{$\begin{array}{c}\text { Zeeman } \\
\text { type }\end{array}$} & \multirow{3}{*}{$\begin{array}{c}\text { Wave- } \\
\text { length } \\
\text { in air }\end{array}$} & \multicolumn{2}{|c|}{ Intensity } & \multirow{3}{*}{ Spectrum } & \multirow{3}{*}{$\begin{array}{c}\text { Zeeman } \\
\text { type }\end{array}$} & \multirow{3}{*}{$\begin{array}{c}\text { Wave- } \\
\text { length } \\
\text { in air }\end{array}$} & Inte & nsity & & \\
\hline & Meggers & Thompson & & & & Meggers & Thompson & & & & Meggers & Thompson & spectrum & type \\
\hline & tamp & tamp & & & & lamp & lamp & & & & lamp & lamp & & \\
\hline 6029.76 & & 1 & II & & 5965.48 & 1 & & I & & 5928.63 & 2 & & I & \\
\hline 6029.05 & $7 \mathrm{~h}$ & & I & & 5964.12 & & 1 & II & & 5928.24 & & 2 & II & \\
\hline 6027.50 & $1 \mathrm{~h}$ & & I & & 5963.60 & 2 & & I & & 5927.69 & 60 & & I & \\
\hline 6024.08 & 40 & 400 & II & 7 & 5962.51 & & $2 \mathrm{e}$ & III & & 5926.958 & 10 & & I & \\
\hline 6023.82 & $2 \mathrm{hl}$ & & I & & 5961.83 & 1 & 60 & II & 4 & 5926.688 & 5 & & I & \\
\hline 6021.92 & 10 & 400 & II & 6 & 5960.20 & 1 & & I & & 5925.46 & 600 & 5 & I & 2 \\
\hline 6021.24 & & $5 \mathrm{e}$ & III & & 5960.04 & 10 & & I & & 5925.00 & 60 & & I & \\
\hline 6020.55 & 40 & 400 & II & & 5959.33 & 1000 & 10 & I & 2 & 5924.498 & 15 & & I & \\
\hline 6018.16 & $5 \mathrm{~h}$ & & I & & 5959.03 & & 7 & II & & 5924.165 & 2 & & I & \\
\hline 6017.70 & & 2 & II & & 5958.70 & 4000 & 100 & I & 1 & 5922.991 & 5 & & I & \\
\hline 6016.62 & 5 & 50 & II & & 5956.86 & & 7 & II & & 5922.153 & 15 & & I & \\
\hline 6014.95 & 1500 & 10 & I & 1 & 5955.34 & 1500 & 8 & I & 2 & 5920.98 & & 2 & II & \\
\hline 6014.16 & 10 & & I & & 5954.19 & $2 \mathrm{~h}$ & & I & & 5920.48 & 2 & & I & \\
\hline 6012.51 & & 9 & II & & 5950.93 & 5 & 250 & II & 6 & 5920.38 & 5 & 300 & II & 6 \\
\hline 6012.16 & 1 & 15 & II & & 5950.66 & 1500 & 10 & I & 3 & 5919.710 & 8 & & I & \\
\hline 6011.55 & & 8 & II & & 5949.83 & & 20 & II & & 5919.585 & 2 & & I & \\
\hline 6011.18 & 1 & & I & & 5949.02 & & $10 \mathrm{~d}$ & III & & 5919.10 & & 7 & II & \\
\hline 6009.56 & 1 & 10 & II & & 5948.98 & & 5 & II & & 5917.826 & 3 & & I & \\
\hline 6008.80 & 5 & 50 & II & & 5947.71 & 2 & & I & & 5917.130 & 6 & & I & \\
\hline 6008.44 & 200 & 1 & I & & 5947.54 & 1 & & I & & 5916.95 & & 2 & II & \\
\hline 6007.41 & 20 & 1000 & II & 4 & 5947.26 & 15 & 500 & II & 5 & 5916.00 & $5 \mathrm{~h} \mathrm{l}$ & & I & \\
\hline 6004.52 & 2000 & 20 & I & 3 & 5946.79 & $4 \mathrm{~h} \mathrm{l}$ & & I & & 5915.052 & 3 & & I & \\
\hline 6004.015 & & 10 & II & & 5946.23 & 1 & & I & & 5914.912 & 2 & 20 & II & \\
\hline 6003.62 & 400 & 10 & I & 1 & 5946.00 & 40 & 2000 & II & 4 & 5914.415 & 5 & & I & \\
\hline 6003.33 & $2 \mathrm{~h} \mathrm{l}$ & & I & & 5945.44 & 8 & & I & & 5912.35 & $5 \mathrm{~h} \mathrm{l}$ & & I & \\
\hline 6002.52 & 3 & 90 & II & & 5945.33 & 3 & & I & & 5912.15 & 2 & & I & \\
\hline 6001.05 & 1 & 30 & II & 6 & 5945.27 & 3 & & I & & 5911.55 & 5 & & I & \\
\hline 6000.91 & & 8 & II & & 5945.10 & 4 & & I & & 5911.35 & 20 & & I & \\
\hline 6000.28 & 20 & & I & & 5944.93 & 2 & & I & & 5910.10 & 4 & & I & \\
\hline 5999.95 & 1 & 30 & II & & 5944.73 & 4 & & I & & 5909.33 & 2 & 10 & II & \\
\hline 5998.71 & & 25 & II & & 5944.63 & 1 & 10 & II & & 5909.10 & 2 & & I & \\
\hline 5996.84 & & 1 & II & & 5944.41 & 3 & & I & & 5908.36 & 400 & 1500 & II & 6 \\
\hline 5993.95 & & 1 & II & & 5944.24 & 2 & & I & & 5906.45 & 2 & & I & \\
\hline 5993.704 & & 15 & II & & 5943.63 & 9 & & I & & 5906.33 & & 2 & II & \\
\hline 5991.51 & 2000 & 6000 & II & 6 & 5943.55 & 5 & 200 & II & & 5905.85 & 2 & & I & \\
\hline 5990.01 & & 10 & II & & 5943.05 & 20 & 250 & II & & 5905.22 & 3 & & I & \\
\hline 5989.33 & 5000 & 100 & I & 3 & 5942.89 & 3 & & I & & 5903.38 & 3 & 90 & II & \\
\hline 5987.91 & 15 & 500 & II & & 5942.73 & 3 & & I & & 5902.80 & & 3 & II & \\
\hline 5987.58 & 20 & & I & & 5942.02 & 4 & & I & & 5902.71 & 2 & & I & \\
\hline 5986.78 & 1 & 40 & II & & 5941.72 & 2 & & I & & 5900.85 & & 20 & II & \\
\hline 5985.98 & & $6 \mathrm{e}$ & III & & 5941.29 & & 2 & II & & 5899.30 & 2 & & I & \\
\hline 5985.35 & 20 & 300 & II & 5 & 5941.04 & 1 & & I & & 5898.77 & 15 & 800 & II & 4 \\
\hline 5984.98 & $3 \mathrm{hl}$ & & I & & 5940.68 & 20 & & I & & 5897.21 & 50 & 2500 & II & 7 \\
\hline 5983.12 & 2 & & I & & 5940.63 & & 1 & II & & 5896.63 & & $6 \mathrm{~d}$ & II & \\
\hline 5980.83 & 2 & & I & & 5939.35 & 2 & 8 & II & & 5895.16 & 2 & & I & \\
\hline 5978.35 & 2 & & I & & 5938.15 & 1 & 10 & II & & 5894.98 & 5 & & I & \\
\hline 5978.11 & & 10 & II & & 5936.68 & & 20 & II & & 5892.30 & & 2 & II & \\
\hline 5976.93 & & 2 & II & & 5936.49 & 900 & 10 & I & 1 & 5888.02 & & 4 & II & \\
\hline 5976.21 & 20 & & I & & 5935.88 & 2 & & I & & 5886.05 & 5 & & I & \\
\hline 5975.70 & 2 & & I & & 5935.56 & 2 & & I & & 5883.61 & & 6 & III & \\
\hline 5975.29 & 2 & & I & & 5935.05 & 20 & 1000 & II & 5 & 5882.80 & 100 & 600 & II & \\
\hline 5974.63 & 2 & & I & & 5934.27 & 3 & & I & & 5877.68 & 10 & & I & \\
\hline 5973.49 & 2 & 20 & II & & 5934.01 & & 1 & II & & 5874.67 & 2 & 200 & II & 7 \\
\hline 5973.05 & & $20 \mathrm{e}$ & III & & 5933.775 & 2 & & I & & 5873.12 & & 10 & II & \\
\hline 5972.73 & 150 & 1 & I & & 5932.98 & 2 & & I & & 5872.90 & & 3 & III & \\
\hline 5972.29 & & 1 & II & & 5932.562 & 4 & & I & & 5872.15 & & 1 & II & \\
\hline 5972.02 & & 2 & II & & 5931.853 & 3 & & I & & 5868.94 & & 7 & II & \\
\hline 5970.26 & & 5 & II & & 5931.73 & $3 \mathrm{~h}$ & & I & & 5868.34 & 2 & 100 & II & 4 \\
\hline 5969.95 & 3 & & I & & 5931.33 & & $3 \mathrm{e}$ & III & & 5866.69 & & 6 & II & \\
\hline 5969.84 & 2 & & I & & 5930.722 & 6 & & I & & 5866.48 & & 1 & II & \\
\hline 5968.23 & $2 \mathrm{~h} \mathrm{l}$ & & I & & 5929.82 & 4 & & I & & 5865.63 & 1 & 70 & II & 6 \\
\hline 5966.86 & 2 & & I & & 5929.77 & & 10 & II & & 5863.86 & & 3 & II & \\
\hline 5966.43 & 20 & & I & & 5928.761 & 5 & & I & & 5863.37 & & 1 & II & \\
\hline
\end{tabular}


TABLE 1.-Emission spectra of ytterbium-Continued

\begin{tabular}{|c|c|c|c|c|c|c|c|c|c|c|c|c|c|c|}
\hline \multirow{3}{*}{$\begin{array}{l}\text { Wave- } \\
\text { length } \\
\text { in air } \\
\circ \\
\end{array}$} & \multicolumn{2}{|c|}{ Intensity } & \multirow{3}{*}{ Spectrum } & \multirow{3}{*}{$\begin{array}{c}\text { Zeeman } \\
\text { type }\end{array}$} & \multirow{3}{*}{$\begin{array}{c}\text { Wave- } \\
\text { length } \\
\text { in air }\end{array}$} & \multicolumn{2}{|c|}{ Intensity } & \multirow{3}{*}{ Spectrum } & \multirow{3}{*}{$\begin{array}{c}\text { Zeeman } \\
\text { type }\end{array}$} & \multirow{3}{*}{$\begin{array}{c}\text { Wave- } \\
\text { length } \\
\text { in air } \\
\AA \\
\end{array}$} & Inte & nsity & & \\
\hline & Meggers & Thompson & & & & Meggers & Thompson & & & & Meggers & & Dperion & type \\
\hline & lamp & lamp & & & & lamp & lamp & & & & lamp & lamp & & \\
\hline 5862.90 & 1 & 15 & II & & 5786.09 & 400 & 3 & I & & 5712.844 & & 15 & II & \\
\hline 5861.53 & & 3 & III & & 5784.43 & $3 \mathrm{~h}$ & & I & & 5711.38 & 1 & & I & \\
\hline 5861.11 & 10 & & I & & 5784.026 & 10 & & I & & 5710.733 & & 25 & II & \\
\hline 5854.89 & & 3 & II & & 5779.92 & & $3 \mathrm{e}$ & III & & 5710.403 & 1 & 15 & II & \\
\hline 5854.510 & 4000 & 200 & I & 3 & 5777.95 & 3 & 100 & II & 5 & 5709.715 & & 2 & II & \\
\hline 5854.30 & 5 & 70 & II & & 5777.29 & 1 & 10 & II & & 5706.773 & 10 & 100 & II & \\
\hline 5853.84 & & 3 & II & & 5775.61 & 1 & 50 & II & & 5704.98 & 2 & 70 & II & 5 \\
\hline 5852.92 & & 5 & II & & 5774.34 & 500 & 5 & I & & 5704.14 & & 3 & II & \\
\hline 5847.18 & 1 & 40 & II & 6 & 5773.82 & 1000 & 10 & I & & 5701.922 & 250 & 3 & I & 3 \\
\hline 5845.50 & $6 \mathrm{~h}$ & & I & & 5772.76 & & 3 & II & & 5699.95 & 3000 & 50 & I & 1 \\
\hline 5844.97 & & $9 \mathrm{e}$ & III & & 5772.39 & $3 \mathrm{~h} \mathrm{l}$ & & I & & 5699.02 & & 15 & II & \\
\hline 5842.44 & 80 & 1 & I & & 5771.66 & 500 & 2000 & II & 4 & 5698.21 & 2 & & I & \\
\hline 5841.96 & 20 & & I & & 5769.44 & & 6 & II & & 5697.65 & & 2 & II & \\
\hline 5839.56 & & 1 & II & & 5767.95 & 3 & 30 & II & & 5696.93 & 2 & & I & \\
\hline 5839.25 & $2 \mathrm{~h}$ & & I & & 5767.20 & 20 & 800 & II & 6 & 5696.717 & & 5 & II & \\
\hline 5838.91 & $6 \mathrm{~h}$ & & I & & 5765.21 & $5 \mathrm{~h}$ & & I & & 5695.22 & & 2 & II & \\
\hline 5838.62 & 2 & 70 & II & 5 & 5764.65 & 1 & 40 & II & & 5694.906 & & 15 & II & \\
\hline 5838.27 & 70 & 1 & I & & 5760.18 & 30 & 6 & II & & 5694.13 & & 1 & II & \\
\hline 5837.14 & 600 & 4000 & II & 6 & 5759.84 & $4 \mathrm{~h}$ & & I & & 5693.694 & 10 & 400 & II & 7 \\
\hline 5836.18 & 1 & 50 & II & & 5758.25 & 9 & & I & & 5689.917 & 1000 & 30 & I & 7 \\
\hline 5835.53 & 1 & 30 & II & & 5757.84 & 2 & 15 & II & & 5689.67 & 3 & 70 & II & \\
\hline 5834.58 & 200 & 3 & I & & 5755.89 & 3000 & 40 & I & 3 & 5688.48 & 200 & 3 & I & \\
\hline 5833.99 & $5 \mathrm{~h}$ & $200 \mathrm{~h}$ & II & & 5754.80 & 2 & 70 & II & & 5687.14 & $1 \mathrm{~d}$ & $20 \mathrm{~d}$ & II & \\
\hline 5833.62 & 20 & & I & & 5753.57 & 1 & 25 & II & & 5686.53 & 80 & 800 & II & 6 \\
\hline 5832.12 & & $2 \mathrm{e}$ & III & & 5753.20 & 2 & & I & & 5685.832 & 1 & 9 & II & \\
\hline 5831.82 & 300 & 3 & I & 3 & 5750.62 & & 8 & II & & 5684.13 & 2 & & I & \\
\hline 5831.58 & & 5 & II & & 5749.91 & 1000 & 400 & II & 3 & 5683.56 & 10 & 300 & II & \\
\hline 5831.04 & & 15 & II & & 5746.703 & & 3 & II & & 5683.09 & 500 & 5 & I & 7 \\
\hline 5830.82 & 2 & & I & & 5746.36 & 1 & 15 & II & & 5679.270 & 1 & 15 & II & \\
\hline 5829.63 & 200 & 2 & I & & 5745.80 & 2000 & 30 & I & 1 & 5679.180 & 2 & 8 & II & \\
\hline 5829.11 & $5 \mathrm{~h}$ & & I & & 5741.99 & 15 & & I & & 5675.180 & 2 & 50 & II & \\
\hline 5828.49 & & 1 & II & & 5741.75 & & 2 & II & & 5674.31 & 10 & 150 & II & 7 \\
\hline 5824.33 & 2 & 50 & II & 7 & 5740.83 & & $15 \mathrm{e}$ & III & & 5672.37 & & 2 & II & \\
\hline 5823.36 & & 2 & II & & 5738.82 & & 5 & II & & 5672.16 & & 8 & II & \\
\hline 5820.17 & 2 & & I & & 5738.53 & 2 & & I & & 5671.45 & & $2 \mathrm{e}$ & III & \\
\hline 5819.99 & & 3 & II & & 5737.09 & & 20 & II & & 5670.02 & 500 & 2 & I & \\
\hline 5819.41 & 50 & 1500 & II & 4 & 5735.773 & 10 & 600 & II & 7 & 5665.34 & & 1 & II & \\
\hline 5814.76 & & 2 & II & & 5735.50 & & 5 & II & & 5663.21 & 200 & 2 & I & \\
\hline 5813.58 & 3 & 40 & II & 5 & 5734.26 & & 3 & II & & 5660.152 & & 4 & II & \\
\hline 5812.64 & $3 \mathrm{~h}$ & & I & & 5733.00 & $2 \mathrm{~h} \mathrm{l}$ & & I & & 5658.86 & & $1 \mathrm{e}$ & III & \\
\hline 5811.90 & 1 & 40 & II & & 5732.60 & $4 \mathrm{~h} \mathrm{l}$ & & I & & 5654.27 & 2 & 150 & II & 7 \\
\hline 5811.13 & 1 & 20 & II & & 5731.94 & $6 \mathrm{~h} \mathrm{l}$ & & I & & 5653.24 & 3 & 200 & II & 7 \\
\hline 5810.67 & 3000 & 7 & I & 1 & 5731.69 & & 3 & II & & 5651.985 & 1000 & 4000 & II & 4 \\
\hline 5808.66 & 3 & 120 & II & & 5731.26 & $6 \mathrm{hl}$ & & I & & 5642.446 & 40 & & I & \\
\hline 5807.86 & & 30 & II & & 5730.41 & $7 \mathrm{~h} \mathrm{l}$ & & I & & 5637.812 & 80 & 600 & II & 6 \\
\hline 5807.51 & 1 & 7 & II & & 5730.001 & 20 & 1500 & II & 5 & 5637.33 & 1 & 6 & II & \\
\hline 5806.58 & 7 & & I & & 5729.26 & $5 \mathrm{~h} \mathrm{l}$ & & I & & 5634.45 & & 1 & II & \\
\hline 5804.06 & 80 & 1 & I & & 5728.853 & 150 & 5 & I & & 5633.81 & & $1 \mathrm{e}$ & III & \\
\hline 5803.44 & 5000 & 80 & I & 2 & 5728.58 & $5 \mathrm{~h} \mathrm{l}$ & & I & & 5631.496 & 2 & 90 & II & \\
\hline 5802.36 & 200 & 1 & I & & 5727.47 & 90 & & I & & 5628.958 & 10 & & I & \\
\hline 5801.50 & & 8 & II & & 5727.21 & & 5 & II & & 5627.89 & 4 & 200 & II & 7 \\
\hline 5801.13 & 1 & 15 & II & & 5726.68 & $10 \mathrm{~h}$ & & I & & 5625.92 & 20 & & I & \\
\hline 5801.04 & 1 & 10 & II & & 5726.49 & & 2 & II & & 5624.494 & & 10 & II & \\
\hline 5799.57 & 1 & 40 & II & & 5724.58 & 500 & 2 & I & 7 & 5620.88 & 1 & 70 & II & \\
\hline 5798.82 & $2 \mathrm{~h}$ & & 1 & & 5724.21 & & 3 & II & & 5620.19 & 50 & 700 & II & 4 \\
\hline 5796.09 & 80 & & I & & 5723.70 & 600 & 7 & I & & 5618.55 & & 1 & II & \\
\hline 5792.28 & 1 & 10 & II & & 5722.743 & & 8 & II & & 5616.66 & $2 \mathrm{~h}$ & & I & \\
\hline 5789.93 & 1500 & 15 & I & & 5719.99 & 20000 & 1000 & I & 3 & 5614.68 & 1 & & I & \\
\hline 5789.63 & 100 & & I & & 5718.86 & & 1 & III & & 5614.116 & 3 & & I & \\
\hline 5789.17 & 2 & 50 & II & & 5717.25 & 10 & 300 & II & 7 & 5613.39 & $5 \mathrm{~h}$ & & I & \\
\hline 5788.74 & 1 & 15 & II & & 5716.70 & & 2 & II & & 5611.50 & 1 & & I & \\
\hline 5787.86 & 500 & 5 & I & & 5713.73 & 20 & 500 & II & 4 & 5610.60 & 2 & & I & \\
\hline 5786.59 & 20 & 150 & II & 5 & 5713.270 & 4 & 15 & II & & 5610.281 & 3 & & I & \\
\hline
\end{tabular}


TABLE 1.-Emission spectra of ytterbium-Continued

\begin{tabular}{|c|c|c|c|c|c|c|c|c|c|c|c|c|c|c|}
\hline \multirow{3}{*}{$\begin{array}{c}\text { Wave- } \\
\text { length } \\
\text { in air } \\
\AA \\
\end{array}$} & \multicolumn{2}{|c|}{ Intensity } & \multirow{3}{*}{ Spectrum } & \multirow{3}{*}{$\begin{array}{c}\text { Zeeman } \\
\text { type }\end{array}$} & \multirow{3}{*}{$\begin{array}{c}\text { Wave- } \\
\text { length } \\
\text { in air } \\
\circ \\
\end{array}$} & \multicolumn{2}{|c|}{ Intensity } & \multirow{3}{*}{ Spectrum } & \multirow{3}{*}{$\begin{array}{c}\text { Zeeman } \\
\text { type }\end{array}$} & \multirow{3}{*}{$\begin{array}{c}\text { Wave- } \\
\text { length } \\
\text { in air } \\
\AA\end{array}$} & Inte & nsity & & \\
\hline & Meggers & Thompson & & & & Meggers & Thompson & & & & Meggers & Thompson & spectrom & type \\
\hline & lamp & lamp & & & & lamp & lamp & & & & lamp & lamp & & \\
\hline 5609.89 & & 1 & II & & 5529.92 & 4 & 150 & II & 7 & 5458.088 & 2 & 60 & II & \\
\hline 5609.44 & 1 & & I & & 5529.08 & 5 & 250 & II & 5 & 5457.427 & 4 & 150 & II & \\
\hline 5608.914 & 1 & 50 & II & & 5528.403 & 3 & & I & & 5455.046 & 5 & 250 & II & 4 \\
\hline 5608.64 & & 2 & II & & 5527.81 & 50 & & I & & 5454.007 & 2500 & 25 & I & 1 \\
\hline 5608.041 & 6 & & I & & 5526.37 & 5 & & I & & 5453.47 & 60 & 1 & I & \\
\hline 5607.91 & & 1 & II & & 5524.544 & 3000 & 30 & I & 7 & 5449.84 & & $\checkmark 1$ & II & \\
\hline 5607.345 & 10 & 150 & II & & 5521.76 & 10 & & I & & 5449.270 & 500 & 2500 & II & 5 \\
\hline 5607.124 & & 4 & II & & 5521.61 & & 3 & II & & 5448.565 & & 4 & II & \\
\hline 5606.64 & & 1 & II & & 5520.22 & 1 & 50 & II & 6 & 5445.492 & 1 & 40 & II & \\
\hline 5605.847 & 3 & & I & & 5519.83 & & 2 & II & & 5441.615 & 2 & 5 & II & \\
\hline 5604.145 & & 9 & II & & 5518.352 & 70 & 1 & I & & 5441.259 & 200 & 1 & I & \\
\hline 5602.35 & 2 & 20 & II & & 5516.334 & & 10 & II & & 5440.548 & 10 & 200 & II & \\
\hline 5602.264 & 3 & 40 & II & & 5515.38 & & 3 & II & & 5438.01 & 2 & & I & \\
\hline 5600.45 & 10 & & I & & 5514.79 & 1 & & I & & 5435.27 & & 6 & II & \\
\hline 5598.14 & 2 & & I & & 5512.47 & 20 & & I & & 5432.89 & 400 & 6 & I & \\
\hline 5597.189 & 800 & 10 & I & 7 & 5512.19 & 20 & & I & & 5432.71 & 60 & 1500 & II & 4 \\
\hline 5595.84 & 5 & & I & & 5511.764 & 1 & 30 & II & 7 & 5431.167 & 2 & 80 & II & \\
\hline 5591.19 & 10 & & I & & 5511.401 & 2 & 70 & II & 7 & 5429.838 & & 8 & II & \\
\hline 5590.10 & 10 & & I & & 5509.198 & & 20 & II & & 5428.162 & & $2 \mathrm{e}$ & III & \\
\hline 5588.452 & 500 & 4000 & II & 4 & 5509.046 & & 3 & II & & 5426.867 & 30 & 900 & II & 7 \\
\hline 5587.249 & 200 & 2 & I & & 5508.266 & & 20 & II & 5 & 5425.445 & 100 & 1 & I & \\
\hline 5586.362 & 2000 & 100 & I & 3 & 5.507 .70 & & 1 & II & & 5424.627 & 30 & 300 & II & 5 \\
\hline 5585.426 & 400 & 5 & I & & 5506.11 & 1500 & 5 & I & & 5422.715 & & 1 & II & \\
\hline 5585.248 & & 20 & II & & 5505.49 & 20000 & 300 & I & 2 & 5421.361 & 7 & & I & \\
\hline 5583.57 & & 1 & II & & 5504.17 & 60 & & I & & 5420.608 & & 7 & II & \\
\hline 5582.236 & & 2 & II & & 5504.026 & 300 & 3 & I & & 5417.421 & & 9 & II & \\
\hline 5581.778 & & 8 & II & & 5502.802 & 1 & 10 & II & & 5414.258 & 6 & 200 & II & 7 \\
\hline 5581.05 & 2 & 40 & II & & 5501.37 & 2 & 20 & II & & 5413.195 & 15 & & I & \\
\hline 5580.79 & 20 & 700 & II & 4 & 5499.65 & & 2 & II & & 5411.038 & 1 & 40 & II & \\
\hline 5578.483 & & 4 & II & & 5498.75 & 5000 & 500 & I & 7 & 5410.635 & & 15 & II & \\
\hline 5578.232 & 2000 & 20 & I & 7 & 5498.390 & & 3 & II & & 5409.473 & 2 & 60 & II & 4 \\
\hline 5576.250 & 5 & & I & & 5494.58 & & 1 & II & & 5408.348 & 100 & 2 & I & \\
\hline 5575.705 & 10 & 100 & II & & 5494.364 & 1 & 10 & II & & 5408.092 & & 4 & II & \\
\hline 5573.64 & & 1 & II & & 5493.088 & 700 & 5 & I & 2 & 5405.541 & 60 & 1 & I & \\
\hline 5572.528 & 30 & 300 & II & & 5491.285 & 6 & & I & & 5404.899 & 300 & 5 & I & \\
\hline 5568.11 & 10000 & 100 & I & 2 & 5490.847 & & 3 & II & & 5403.079 & 3000 & 100 & I & 1 \\
\hline 5566.04 & & 3 & II & & 5489.633 & 80 & & I & & 5402.706 & & 3 & II & \\
\hline 5564.352 & & 3 & II & & 5488.883 & & 8 & II & & 5402.00 & 150 & 2 & I & \\
\hline 5562.093 & 2000 & 200 & I & 3 & 5487.512 & 1 & 9 & II & & 5399.71 & 50 & 500 & II & 7 \\
\hline 5559.58 & & 1 & II & & 5486.535 & 7 & 150 & II & 6 & 5398.084 & & $2 \mathrm{e}$ & III & \\
\hline 5558.98 & 800 & 8 & I & & 5483.212 & & 3 & II & & 5397.23 & & 1 & II & \\
\hline 5556.466 & $50000 \mathrm{~A}$ & 10000 & I & 2 & 5481.925 & 20000 & 400 & I & 2 & 5395.73 & 20 & 300 & II & 7 \\
\hline 5554.62 & 500 & 5 & I & & 5479.806 & 1 & 50 & II & & 5394.084 & & 3 & II & \\
\hline 5554.30 & & 4 & II & & 5479.122 & & $2 \mathrm{~d}$ & II & & 5393.757 & 2500 & 90 & I & 1 \\
\hline 5552.30 & & $1 \mathrm{e}$ & III & & 5478.50 & 100 & 1500 & II & 4 & 5393.37 & 5 & 150 & II & 6 \\
\hline 5551.69 & & 2 & II & & 5477.206 & & 9 & II & & 5390.845 & 2500 & 40 & I & 3 \\
\hline 5550.37 & & 7 & II & & 5476.90 & 2 & & I & & 5390.622 & 4000 & 150 & I & 1 \\
\hline 5549.79 & & 20 & II & & 5475.706 & & 10 & II & & 5390.18 & & 6 & II & \\
\hline 5549.32 & & 1 & II & & 5475.132 & & 4 & II & & 5389.84 & 200 & 800 & II & 5 \\
\hline 5548.918 & & 50 & II & & 5474.565 & 400 & 5 & I & & 5387.99 & 90 & 8 & I & \\
\hline 5548.79 & 100 & & I & & 5474.037 & 1500 & 15 & I & 1 & 5386.72 & 1 & 50 & II & \\
\hline 5547.16 & 50 & 500 & II & 7 & 5471.17 & 30 & 400 & II & 5 & 5386.07 & & 10 & II & \\
\hline 5545.814 & 500 & 10 & I & & 5469.353 & 400 & 5 & I & & 5385.29 & 200 & 2 & I & \\
\hline 5544.17 & & $1 \mathrm{~h}$ & II & & 5468.387 & 150 & 2 & I & & 5384.48 & 5 & 50 & II & 5 \\
\hline 5543.96 & & 8 & II & & 5468.260 & & 3 & II & & 5383.95 & 3 & 60 & II & \\
\hline 5540.03 & & 10 & II & & 5468.099 & 1 & & I & & 5382.55 & 2 & & I & \\
\hline 5539.053 & 20000 & 1000 & I & 1 & 5467.495 & 1 & 9 & II & & 5380.94 & 1 & & I & \\
\hline 5538.016 & 50 & & I & & 5466.714 & 1 & 25 & II & & 5380.54 & 500 & 7 & I & 2 \\
\hline 5535.47 & 7 & & I & & 5464.894 & 6 & 200 & II & 7 & 5380.24 & 70 & 2 & I & 7 \\
\hline 5533.21 & & 7 & II & & 5463.850 & & 3 & II & & 5379.15 & 6 & & I & \\
\hline 5533.14 & & 10 & II & & 5463.24 & 4 & & I & & 5376.97 & 5 & 250 & II & 7 \\
\hline 5532.111 & & 3 & II & & 5463.12 & & 10 & II & & 5371.53 & & 4 & II & \\
\hline 5530.73 & 1 & 1 & II & & 5460.74 & & $4 \mathrm{e}$ & III & & 5370.47 & & 9 & II & \\
\hline
\end{tabular}


TABLE 1.-Emission spectra of ytterbium-Continued

\begin{tabular}{|c|c|c|c|c|c|c|c|c|c|c|c|c|c|c|}
\hline \multirow{3}{*}{$\begin{array}{c}\begin{array}{c}\text { Wave- } \\
\text { length } \\
\text { in air }\end{array} \\
\AA \\
\end{array}$} & \multicolumn{2}{|c|}{ Intensity } & \multirow{3}{*}{ Spectrum } & \multirow{3}{*}{$\begin{array}{c}\text { Zeeman } \\
\text { type }\end{array}$} & \multirow{3}{*}{$\begin{array}{c}\text { Wave- } \\
\text { length } \\
\text { in air }\end{array}$} & \multicolumn{2}{|c|}{ Intensity } & \multirow{3}{*}{ Spectrum } & \multirow{3}{*}{$\begin{array}{c}\text { Zeeman } \\
\text { type }\end{array}$} & \multirow{3}{*}{$\begin{array}{c}\text { Wave- } \\
\text { length } \\
\text { in air }\end{array}$} & Inte & nsity & & \\
\hline & Meggers & Thompson & & & & Meggers & Thompson & & & & Meggers & Thompson & 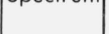 & type \\
\hline & lamp & lamp & & & & $\operatorname{lamp}$ & lamp & & & & lamp & lamp & & \\
\hline 5368.28 & 50 & 400 & II & 4 & 5290.578 & 150 & 3 & I & & 5218.792 & 1 & & I & \\
\hline 5367.78 & 10 & 100 & II & & 5290.08 & 2 & 20 & II & & 5217.936 & 3 & 70 & II & 7 \\
\hline 5367.35 & 4 & 60 & II & 7 & 5289.45 & & 2 & II & & 5215.44 & 4 & 100 & II & \\
\hline 5365.41 & 1 & 50 & II & & 5289.178 & 1 & 5 & II & & 5214.95 & 500 & 5 & I & 3 \\
\hline 5364.78 & & 15 & II & & 5288.51 & 2000 & 15 & I & 7 & 5212.232 & & 25 & II & \\
\hline 5364.12 & & 15 & II & & 5287.45 & 3000 & 70 & I & 1 & 5211.604 & 6000 & 400 & I & 3 \\
\hline 5363.66 & 10000 & 200 & I & 2 & 5287.285 & 5 & 100 & II & & 5209.54 & & 1 & II & \\
\hline 5362.43 & 1 & 20 & II & & 5286.12 & 2 & 100 & II & & 5205.574 & 4 & 30 & II & \\
\hline 5360.85 & & 1 & II & & 5283.825 & & 3 & II & & 5204.57 & & 1 & II & \\
\hline 5360.10 & 1 & 30 & II & & 5283.00 & & 1 & II & & 5202.87 & & 1 & II & \\
\hline 5359.91 & 6 & 200 & II & & 5281.210 & & 20 & II & & 5201.32 & & 1 & II & \\
\hline 5358.64 & 400 & 2000 & II & 6 & 5280.522 & 1 & 50 & II & & 5201.21 & & 2 & II & \\
\hline 5357.71 & & 3 & II & & 5279.53 & 1000 & 5000 & II & 4 & 5200.74 & & 4 & II & \\
\hline 5357.08 & 20 & 200 & II & & 5277.04 & 30000 & 3000 & I & 2 & 5200.54 & 50 & 500 & II & 4 \\
\hline 5356.54 & 1 & 2 & II & & 5275.592 & 1000 & 10 & I & 3 & 5200.10 & 4 & & I & \\
\hline 5356.41 & & 10 & II & & 5271.473 & 400 & 20 & I & & 5199.14 & & 1 & II & \\
\hline 5352.95 & 1000 & 6000 & II & 4 & 5266.964 & 15 & & I & & 5196.81 & 40 & & I & \\
\hline 5351.29 & 5000 & 300 & I & 3 & 5265.63 & & 8 & II & & 5196.085 & 5000 & 200 & 1 & 3 \\
\hline 5350.474 & 2 & 40 & II & & 5265.558 & 40 & & I & & 5195.20 & 100 & 1 & I & \\
\hline 5349.626 & & 5 & II & & 5264.238 & 10 & & I & & 5194.752 & 2000 & 80 & I & \\
\hline 5349.10 & 1 & 6 & II & & 5263.570 & 6 & 200 & II & 6 & 5193.850 & 2000 & 20 & 1 & 1 \\
\hline 5348.208 & 300 & 3 & I & & 5262.012 & 1 & 30 & II & & 5189.89 & & 7 & II & \\
\hline 5347.22 & 1000 & 3000 & II & 4 & 5261.52 & & 1 & II & & 5189.50 & & 8 & II & \\
\hline 5345.83 & 200 & 900 & II & & 5259.65 & & 1 & II & & 5189.14 & 200 & 2 & I & \\
\hline 5345.66 & 400 & 1500 & II & & 5258.165 & 800 & 10 & I & & 5188.89 & & 9 & II & \\
\hline 5342.99 & & 7 & II & & 5257.490 & 800 & 3000 & II & 6 & 5187.33 & & 3 & II & \\
\hline 5341.10 & 2 & & I & & 5257.29 & 90 & & 1 & & 5185.15 & & 1 & II & \\
\hline 5339.69 & & 1 & II & & 5256.85 & & 20 & II & & 5184.66 & & 1 & II & \\
\hline 5339.312 & & 15 & II & & 5255.680 & 4 & 150 & II & & 5184.15 & 150 & 1000 & II & 4 \\
\hline 5338.75 & 10 & 100 & II & 7 & 5255.61 & 2 & & I & & 5182.755 & 2000 & 50 & I & 7 \\
\hline 5338.31 & 60 & & I & & 5254.79 & & $2 \mathrm{e}$ & III & & 5180.641 & & $5 \mathrm{e}$ & III & \\
\hline 5337.11 & & 7 & II & & 5254.60 & & 3 & II & & 5180.355 & 15 & 150 & II & 6 \\
\hline 5335.82 & 10 & & I & & 5254.20 & 1 & 20 & II & & 5178.73 & 60 & & I & \\
\hline 5335.49 & 50 & & I & & 5253.448 & 1 & & I & & 5178.70 & 90 & 20 & I & \\
\hline 5335.15 & 2000 & 7000 & II & 4 & 5250.804 & 100 & 2 & I & & 5175.45 & 250 & 2 & I & \\
\hline 5334.02 & 100 & 200 & II & & 5250.51 & 1 & 8 & II & & 5173.11 & 2 & 200 & II & \\
\hline 5331.536 & & 20 & II & & 5249.80 & 5 & 150 & II & & 5173.064 & 10 & 100 & II & \\
\hline 5330.92 & & 1 & II & & 5248.99 & & 6 & II & & 5172.40 & & 1 & II & \\
\hline 5330.79 & 1 & & I & & 5248.17 & & 1 & II & & 5172.214 & 50 & 1 & I & \\
\hline 5330.348 & 6 & & I & & 5247.94 & 15 & & I & & 5168.29 & 90 & & I & \\
\hline 5328.526 & 3 & 50 & II & & 5246.857 & 400 & 4 & I & & 5167.660 & & 3 & II & \\
\hline 5327.593 & 4 & & I & & 5246.39 & 4 & 40 & II & & 5163.862 & 1 & & I & \\
\hline 5324.778 & & 7 & II & & 5244.61 & 100 & 300 & II & 5 & 5160.99 & & 1 & II & \\
\hline 5324.486 & & 9 & II & & 5244.11 & 10000 & 250 & I & 2 & 5160.74 & & $1 \mathrm{e}$ & III & \\
\hline 5323.10 & 300 & 3 & I & 1 & 5243.56 & & 1 & II & & 5160.47 & 7 & & I & \\
\hline 5322.541 & & 8 & II & & 5243.41 & & 4 & II & & 5159.655 & & 3 & II & \\
\hline 5321.14 & 10 & 250 & II & & 5243.15 & & 2 & II & & 5157.962 & 3 & 100 & II & \\
\hline 5320.962 & 5 & & I & & 5240.51 & 300 & 1000 & II & 6 & 5156.285 & 100 & 2 & I & \\
\hline 5320.774 & & 15 & II & & 5239.25 & & 2 & II & & 5152.34 & 7 & 200 & II & \\
\hline 5320.324 & 6 & & I & & 5236.66 & 3 & 150 & II & & 5151.25 & & 4 & II & \\
\hline 5318.79 & & 1 & II & & 5234.28 & & 2 & II & & 5149.04 & & 1 & II & \\
\hline 5315.462 & & 6 & II & & 5232.537 & 6 & 40 & II & & 5148.75 & 1 & & I & \\
\hline 5309.31 & 30 & 200 & II & 4 & 5230.292 & 60 & & I & & 5147.02 & 50 & 700 & II & 6 \\
\hline 5307.74 & & 6 & II & & 5229.963 & 1 & 60 & II & & 5146.37 & & $3 \mathrm{e}$ & III & \\
\hline 5306.506 & & 40 & II & & 5228.172 & 1000 & 30 & I & 7 & 5142.28 & 3 & 100 & II & \\
\hline 5300.935 & 100 & 2000 & II & 4 & 5227.271 & 4000 & 80 & I & 1 & 5141.64 & & 3 & II & \\
\hline 5299.852 & 2000 & 10 & I & 3 & 5227.01 & 1 & 70 & II & & 5140.38 & 120 & 2 & I & \\
\hline 5297.153 & 150 & 4 & I & & 5226.18 & 20 & 150 & II & & 5140.11 & 20 & & I & \\
\hline 5297.02 & 50 & 1 & I & & 5224.53 & 1 & & I & & 5139.53 & 900 & 10 & I & 1 \\
\hline 5295.755 & & 5 & II & & 5222.68 & & 3 & II & & 5139.35 & 40 & & I & \\
\hline 5293.726 & & 3 & II & & 5221.606 & 50 & 1 & I & & 5138.01 & & 1 & II & \\
\hline 5291.99 & & 10 & II & & 5220.82 & & 5 & II & & 5137.43 & 2 & 4 & II & \\
\hline 5291.077 & & 5 & II & & 5220.41 & & 10 & II & & 5137.389 & 6 & & I & \\
\hline
\end{tabular}


TABLE 1.-Emission spectra of ytterbium-Continued

\begin{tabular}{|c|c|c|c|c|c|c|c|c|c|c|c|c|c|c|}
\hline \multirow{3}{*}{$\begin{array}{c}\text { Wave- } \\
\text { length } \\
\text { in air }\end{array}$} & \multicolumn{2}{|c|}{ Intensity } & \multirow{3}{*}{ Spectrum } & \multirow{3}{*}{$\begin{array}{c}\text { Zeeman } \\
\text { type }\end{array}$} & \multirow{3}{*}{$\begin{array}{l}\text { Wave- } \\
\text { length } \\
\text { in air } \\
\circ \\
\text { A }\end{array}$} & \multicolumn{2}{|c|}{ Intensity } & \multirow{3}{*}{ Spectrum } & \multirow{3}{*}{$\begin{array}{c}\text { Zeeman } \\
\text { type }\end{array}$} & \multirow{3}{*}{$\begin{array}{c}\text { Wave- } \\
\text { length } \\
\text { in air }\end{array}$} & Inte & ensity & & \\
\hline & Meggers & Thompson & & & & & Thompson & & & & Meggers & Thompson & opecirom & type \\
\hline & lamp & lamp & & & & lamp & lamp & & & & lamp & lamp & & \\
\hline 5137.23 & & 1 & II & & 5067.800 & 1500 & 40 & I & 2 & 4992.786 & 2 & & I & \\
\hline 5136.76 & 2 & & I & & 5067.600 & & 10 & II & & 4992.654 & & 6 & II & \\
\hline 5136.49 & & 1 & II & & 5067.297 & 200 & 800 & II & 4 & 4992.520 & & 7 & II & \\
\hline 5135.98 & 200 & 1500 & II & 7 & 5065.74 & & 1 & II & & 4992.102 & 2 & & I & \\
\hline 5133.42 & & 1 & II & & 5065.488 & & 4 & II & & 4989.924 & 6 & & I & \\
\hline 5132.71 & 90 & 1 & I & & 5063.605 & 8 & & I & & 4989.794 & & 4 & II & \\
\hline 5132.40 & 1 & 60 & II & & 5062.938 & 30 & 300 & II & 6 & 4989.57 & 100 & 1 & I & \\
\hline 5131.01 & 1 & 10 & II & & 5062.634 & & 15 & II & & 4988.383 & 1 & 15 & II & \\
\hline 5130.711 & & 2 & II & & 5061.87 & & $2 \mathrm{e}$ & III & & 4988.022 & & 1 & II & \\
\hline 5130.56 & & 1 & If & & 5061.24 & & 1 & II & & 4987.292 & 1 & 15 & II & \\
\hline 5130.523 & 100 & 2 & I & & 5060.722 & 2 & & I & & 4986.88 & 6 & 3 & II & \\
\hline 5130.30 & & $8 \mathrm{e}$ & III & & 5058.71 & & 8 & II & & 4985.36 & 2 & & I & \\
\hline 5130.095 & 7 & & I & & 5058.613 & 900 & 5 & I & 2 & 4982.82 & 2 & & I & \\
\hline 5129.688 & 200 & 2 & I & & 5057.275 & & 2 & II & & 4981.34 & 1 & & I & \\
\hline 5129.56 & & 60 & II & & 5055.933 & 15 & & I & & 4978.62 & 1 & 9 & II & \\
\hline 5128.55 & 3 & 100 & II & & 5055.485 & 2 & & I & & 4976.65 & & 2 & II & \\
\hline 5127.64 & 2 & & I & & 5054.94 & & $15 \mathrm{e}$ & III & & 4975.86 & & $4 \mathrm{e}$ & III & \\
\hline 5126.80 & 2000 & 20 & I & 3 & 5052.90 & & $2 \mathrm{e}$ & III & & 4974.16 & 2000 & 100 & I & 2 \\
\hline 5121.58 & 30 & 300 & II & 6 & 5051.096 & & 2 & II & & 4973.90 & & 15 & II & \\
\hline 5119.29 & 100 & 1 & I & & 5050.77 & 15 & & I & & 4972.42 & 10 & 2 & I & \\
\hline 5117.72 & 1 & 25 & II & & 5049.832 & 5 & 250 & II & 5 & 4972.26 & 1 & 20 & II & \\
\hline 5116.200 & & 10 & II & & 5047.08 & & $15 \mathrm{~d}$ & II & & 4971.20 & & 8 & II & \\
\hline 5114.606 & 40 & 1 & I & & 5043.708 & 150 & 4 & I & 7 & 4970.41 & 40 & 7 & I & \\
\hline 5113.34 & 700 & 10 & I & 3 & 5043.28 & & 1 & II & & 4970.203 & 3 & 4 & II & \\
\hline 5111.89 & 2 & & I & & 5042.982 & 3 & 20 & II & 7 & 4967.35 & & 2 & II & \\
\hline 5111.47 & 1 & 60 & II & & 5041.756 & & 4 & II & & 4966.902 & $10000 \mathrm{R}$ & 700 & I & 3 \\
\hline 5107.58 & & 1 & II & & 5039.86 & & 2 & II & & 4965.43 & 3 & 15 & II & \\
\hline 5106.95 & 1 & & I & & 5038.240 & & 15 & II & & 4964.61 & & $3 \mathrm{e}$ & III & \\
\hline 5106.12 & & 1 & II & & 5034.262 & 5 & 1 & I & & 4963.18 & & 2 & II & \\
\hline 5105.75 & 600 & $30 \mathrm{~h} \mathrm{l}$ & I & 7 & 5033.289 & 1 & 20 & II & & 4962.28 & 1 & & I & \\
\hline 5105.028 & 10 & 150 & II & & 5032.899 & 2 & & I & & 4961.124 & & 3 & II & \\
\hline 5104.85 & 2000 & 30 & I & 1 & 5032.586 & 1 & 25 & II & & 4960.478 & & $4 \mathrm{e}$ & III & \\
\hline 5104.42 & 20 & 400 & II & & 5032.14 & 2 & & I & & 4960.28 & 1 & & I & \\
\hline 5103.724 & 5 & 1 & I & & 5029.342 & & 3 & II & & 4958.895 & & 2 & II & \\
\hline 5103.644 & 5 & & I & & 5029.03 & 6 & & I & & 4957.83 & 8 & & I & \\
\hline 5101.810 & & 3 & II & & 5027.67 & 2000 & 80 & I & 2 & 4957.262 & & 7 & II & \\
\hline 5101.57 & 3 & 80 & II & & 5025.356 & 1 & 6 & II & & 4956.512 & 500 & 9 & I & 3 \\
\hline 5100.08 & 100 & 1 & I & & 5023.925 & 1 & 9 & II & & 4955.98 & & 1 & II & \\
\hline 5099.11 & & 3 & II & & 5022.32 & & $3 \mathrm{e}$ & III & & 4954.68 & 3 & 50 & II & 7 \\
\hline 5097.75 & & 2 & II & & 5021.860 & 400 & 4 & I & & 4953.72 & 1 & & I & \\
\hline 5095.99 & & 3 & II & & 5021.13 & 50 & 400 & II & 7 & 4953.622 & & 3 & II & \\
\hline 5093.92 & 2 & & I & & 5019.691 & 1500 & 15 & I & 1 & 4952.740 & & 2 & II & \\
\hline 5090.77 & 80 & & I & & 5017.80 & & 3 & II & & 4951.88 & 2 & & I & \\
\hline 5090.628 & & 15 & II & & 5014.98 & & 2 & II & & 4949.24 & & 2 & II & \\
\hline 5088.92 & 1 & 30 & II & & 5014.472 & 20 & 250 & II & 6 & 4946.524 & & 1 & II & \\
\hline 5088.275 & 15 & & I & & 5013.76 & & 1 & II & & 4946.19 & & 1 & II & \\
\hline 5087.89 & 4 & & I & & 5013.27 & & 2 & III & & 4945.944 & & 3 & II & \\
\hline 5087.61 & 10 & 150 & II & 4 & 5010.04 & & 1 & II & & 4944.956 & 5 & 150 & II & \\
\hline 5086.45 & 15 & & I & & 5009.525 & 500 & 2000 & II & 7 & 4944.080 & 200 & 600 & II & 6 \\
\hline 5085.70 & 2 & 80 & II & & 5009.025 & & 2 & II & & $49 \div 2.742$ & 20 & 200 & II & \\
\hline 5085.22 & & 1 & II & & 5008.876 & 1 & 15 & II & & 4942.254 & 250 & 3 & I & \\
\hline 5083.938 & 90 & 1 & I & & 5007.76 & & 1 & II & & 4940.53 & & 2 & II & \\
\hline 5083.52 & & 2 & II & & 5005.25 & & 1 & II & & 4938.828 & & 4 & II & \\
\hline 5082.934 & & 7 & II & & 5004.79 & 15 & & I & & 4937.222 & 100 & 2000 & II & 7 \\
\hline 5082.590 & 250 & 9 & I & & 5004.63 & & 1 & II & & 4935.50 & 20000R & 2000 & I & 1 \\
\hline 5080.981 & 500 & 9 & I & 2 & 5003.306 & 2 & & I & & 4932.81 & 5 & & I & \\
\hline 5076.957 & & 6 & II & & 5002.98 & 5 & 70 & II & 5 & 4931.953 & 1000 & 40 & I & 2 \\
\hline 5076.744 & 5000 & 150 & I & 1 & 5000.452 & & 2 & II & & 4931.16 & 5 & & I & \\
\hline 5075.813 & & 8 & II & & 4998.87 & & 3 & II & & 4930.78 & & 1 & II & \\
\hline 5074.609 & & 80 & II & & 4998.266 & 3 & 15 & II & & 4929.31 & 1 & 20 & II & 7 \\
\hline 5074.34 & 20000 & 2000 & I & 1 & 4996.604 & & 4 & II & & 4928.184 & & 4 & II & \\
\hline 5073.35 & & 1 & II & & 4994.74 & & 1 & II & & 4927.154 & & 1 & II & \\
\hline 5069.144 & 4000 & 150 & I & 7 & 4993.32 & & 1 & II & & 4924.187 & & 2 & II & \\
\hline
\end{tabular}


TABLE 1.-Emission spectra of ytterbium-Continued

\begin{tabular}{|c|c|c|c|c|c|c|c|c|c|c|c|c|c|c|}
\hline \multirow{3}{*}{$\begin{array}{c}\begin{array}{c}\text { Wave- } \\
\text { length } \\
\text { in air }\end{array} \\
\AA \\
\end{array}$} & \multicolumn{2}{|c|}{ Intensity } & \multirow{3}{*}{ Spectrum } & \multirow{3}{*}{$\begin{array}{c}\text { Zeeman } \\
\text { type }\end{array}$} & \multirow{3}{*}{$\begin{array}{c}\text { Wave- } \\
\text { length } \\
\text { in air }\end{array}$} & \multicolumn{2}{|c|}{ Intensity } & \multirow{3}{*}{ Spectrum } & \multirow{3}{*}{$\begin{array}{c}\text { Zeeman } \\
\text { type }\end{array}$} & \multirow{3}{*}{$\begin{array}{c}\text { Wave- } \\
\text { length } \\
\text { in air }\end{array}$} & & insity & & \\
\hline & Meggers & Thompson & & & & Meggers & Thompson & & & & Meggers & Thompson & Spectrum & type \\
\hline & lamp & lamp & & & & lamp & lamp & & & & lamp & lamp & & \\
\hline 4923.747 & & 1 & II & & 4845.612 & 3 & & I & & 4778.982 & 200 & 7 & I & 3 \\
\hline 4919.595 & 100 & 2 & I & & 4841.918 & 50 & 2 & I & & 4778.414 & & 1 & II & \\
\hline 4918.45 & & 10 & II & & 4841.47 & & 2 & II & & 4778.35 & & 1 & II & \\
\hline 4918.118 & 150 & 2 & I & 7 & 4841.153 & 50 & 150 & II & 6 & 4777.946 & & 4 & II & \\
\hline 4917.044 & 20 & 60 & II & 4 & 4839.462 & 2 & & I & & 4777.56 & & 1 & II & \\
\hline 4915.42 & & 1 & II & & 4839.279 & 1 & 15 & II & & 4774.145 & 4 & 8 & II & \\
\hline 4912.365 & 3000 & 100 & I & 3 & 4839.014 & 1 & 7 & II & & 4773.839 & & 8 & II & \\
\hline 4909.81 & & 3 & II & & 4838.657 & 7 & & I & & 4773.13 & & 3 & II & \\
\hline 4906.335 & 50 & & I & & 4838.220 & 10 & 20 & II & & 4772.43 & & 2 & II & \\
\hline 4906.27 & & 9 & II & & 4837.46 & 4000 & 100 & I & 2 & 4772.23 & & 6 & II & \\
\hline 4905.48 & & 2 & II & & 4837.045 & 500 & 1000 & II & & 4772.000 & & 2 & II & \\
\hline 4904.93 & 1 & & I & & 4836.96 & 500 & 1000 & II & 6 & 4770.834 & 150 & 2 & I & \\
\hline 4903.89 & 1 & 25 & II & & 4834.926 & 1 & 10 & II & & 4768.32 & & 6 & II & \\
\hline 4903.71 & 3 & 50 & II & 7 & 4834.72 & 10 & 100 & II & 4 & 4768.036 & & 3 & II & \\
\hline 4900.16 & & 4 & II & & 4834.23 & 2 & 3 & II & & 4765.51 & 1 & & I & \\
\hline 4899.980 & & 2 & II & & 4833.55 & & 1 & II & & 4765.080 & 3 & & I & \\
\hline 4899.78 & 500 & 5 & I & 3 & 4833.24 & & 1 & II & & 4762.587 & 300 & 5 & I & 2 \\
\hline 4898.42 & & 15 & II & & 4832.989 & 200 & 5 & I & & 4761.55 & 2 & & 1 & \\
\hline 4897.90 & 2 & 40 & II & & 4831.912 & 400 & 20 & I & & 4761.139 & 2 & 40 & II & \\
\hline 4897.08 & 5 & & I & & 4831.533 & 2 & 20 & II & & 4758.320 & 80 & 4 & I & 7 \\
\hline 4895.606 & 150 & 5 & I & & 4831.30 & 600 & 10 & I & 3 & 4757.559 & 40 & 1 & I & \\
\hline 4894.983 & 50 & 400 & II & 5 & 4830.704 & 500 & 10 & I & 2 & 4757.14 & & 1 & II & \\
\hline 4894.596 & 3000 & 400 & I & 1 & 4828.29 & 3 & & I & & 4756.36 & & 1 & II & \\
\hline 4894.296 & 50 & & 1 & & 4827.81 & & 2 & II & & 4755.15 & 4 & & I & \\
\hline 4893.465 & 500 & 5 & I & 7 & 4826.78 & & 2 & II & & 4754.370 & 5 & & I & \\
\hline 4891.992 & 200 & 4 & I & 7 & 4825.655 & 1 & & I & & 4752.91 & 100 & 400 & II & 6 \\
\hline 4891.654 & & 3 & II & & 4824.28 & 1 & & I & & 4751.789 & 400 & 10 & I & 1 \\
\hline 4891.056 & 1 & 8 & II & & 4823.615 & & 2 & II & & 4751.22 & 3 & & I & \\
\hline 4890.07 & & 1 & II & & 4822.47 & & 1 & II & & 4749.948 & 2 & 30 & II & \\
\hline 4888.99 & & 3 & II & & 4822.010 & & 3 & II & & 4749.55 & & 1 & II & \\
\hline 4888.18 & 25 & 1 & I & & 4821.58 & & $2 \mathrm{e}$ & III & & 4749.06 & 1 & 5 & II & \\
\hline 4887.662 & & 2 & II & & 4820.74 & & 1 & II & & 4748.71 & & 2 & II & \\
\hline 4885.45 & 90 & 3 & I & & 4820.242 & 400 & 1000 & II & 4 & 4748.08 & & $2 \mathrm{~d}$ & II & \\
\hline 4884.205 & & 4 & II & & 4819.59 & 20 & 1 & I & & 4746.68 & 10 & 150 & II & 6 \\
\hline 4882.15 & 2 & & I & & 4818.371 & 100 & 600 & II & 4 & 4745.483 & 1 & 20 & II & \\
\hline 4880.674 & & 8 & II & & 4816.43 & 5000 & 600 & I & 1 & 4745.27 & 2 & & I & \\
\hline 4880.190 & & 10 & II & & 4815.612 & & 6 & II & & 4743.57 & & 3 & II & ! \\
\hline 4875.852 & & 2 & II & & 4815.28 & & 1 & II & & 4743.356 & 250 & 7 & I & 3 \\
\hline 4873.180 & & 2 & II & & 4813.92 & 2 & 7 & II & & 4741.574 & 8 & & I & \\
\hline 4871.670 & 2 & 40 & II & 7 & 4812.918 & 400 & 40 & I & 3 & 4739.70 & 1 & 10 & II & \\
\hline 4871.150 & 10 & 90 & II & 7 & 4811.89 & 1 & & I & & 4739.330 & 1 & & I & \\
\hline 4870.288 & 30 & & I & & 4810.51 & 40 & 4 & I & & 4738.656 & 5 & 7 & II & \\
\hline 4868.511 & 8 & & I & & 4809.451 & 2 & 30 & II & & 4736.89 & 1 & & I & \\
\hline 4863.87 & & 3 & II & & 4808.502 & 2 & 50 & II & & 4736.69 & & 2 & II & \\
\hline 4863.56 & & 1 & II & & 4804.571 & 3 & & I & & 4736.525 & 15 & & I & \\
\hline 4863.295 & 40 & & I & & 4803.653 & & 10 & II & & 4732.935 & 1 & 25 & II & \\
\hline 4862.802 & & $4 \mathrm{e}$ & III & & 4801.473 & & 2 & II & & 4732.505 & 18 & & I & \\
\hline 4862.524 & 2 & 40 & I & & 4800.535 & 2 & & I & & 4732.14 & 2 & & I & \\
\hline 4860.84 & & 2 & II & & 4799.96 & & 1 & II & & 4732.025 & 30 & 200 & II & 6 \\
\hline 4859.49 & & 2 & II & & 4798.72 & & 3 & II & & 4731.70 & & 2 & II & \\
\hline 4857.542 & 50 & 1 & I & & 4795.829 & 80 & 1 & I & & 4731.04 & 2 & & I & \\
\hline 4856.50 & 1 & & I & & 4794.96 & 1 & & I & & 4729.25 & & 3 & II & \\
\hline 4855.922 & 15 & & I & & 4794.85 & 1 & & I & & 4728.109 & & 8 & II & \\
\hline 4854.92 & 1 & & I & & 4794.57 & 1 & & I & & 4727.901 & & 7 & II & \\
\hline 4853.826 & 500 & 7 & I & 1 & 4792.63 & & 3 & II & & 4726.54 & & 1 & II & \\
\hline 4853.347 & & 7 & II & & 4792.336 & & 2 & II & & 4726.08 & 1000 & 3000 & II & 4 \\
\hline 4851.150 & 300 & 600 & II & 7 & 4790.61 & 1 & 15 & II & & 4725.14 & & 2 & II & \\
\hline 4850.848 & 1 & 10 & II & & 4788.924 & & 5 & II & & 4723.85 & 1 & & I & \\
\hline 4849.265 & & 2 & II & & 4786.61 & 2000 & 8000 & II & 4 & 4723.404 & & 4 & II & \\
\hline 4849.034 & 100 & 1 & I & & 4784.54 & 70 & 9 & I & & 4722.53 & 2 & & I & \\
\hline 4848.455 & 50 & 300 & II & & 4781.867 & 10000 & 2000 & I & 1 & 4722.148 & 10 & & I & \\
\hline 4846.873 & 1 & 15 & II & & 4781.17 & & $2 \mathrm{~d}$ & II & & 4720.955 & & $2 \mathrm{e}$ & III & \\
\hline 484.6 .612 & 4 & 20 & II & & 4780.32 & 400 & 15 & I & 1 & 4720.79 & 2000 & 50 & I & 2 \\
\hline
\end{tabular}


TABLE 1.-Emission spectra of ytterbium-Continued

\begin{tabular}{|c|c|c|c|c|c|c|c|c|c|c|c|c|c|c|}
\hline \multirow{3}{*}{$\begin{array}{c}\begin{array}{c}\text { Wave- } \\
\text { length } \\
\text { in air }\end{array} \\
\stackrel{\circ}{\mathrm{A}} \\
\end{array}$} & \multicolumn{2}{|c|}{ Intensity } & \multirow{3}{*}{ Spectrum } & \multirow{3}{*}{$\begin{array}{c}\text { Zeeman } \\
\text { type }\end{array}$} & \multirow{3}{*}{$\begin{array}{c}\text { Wave- } \\
\text { length } \\
\text { in air }\end{array}$} & \multicolumn{2}{|c|}{ Intensity } & \multirow{3}{*}{ Spectrum } & \multirow{3}{*}{$\begin{array}{c}\text { Zeeman } \\
\text { type }\end{array}$} & \multirow{3}{*}{$\begin{array}{c}\begin{array}{c}\text { Wave- } \\
\text { length } \\
\text { in air }\end{array} \\
\AA \\
\end{array}$} & & ensity & & \\
\hline & Meggers & Thompson & & & & Meggers & Thompson & & & & Meggers & Thompson & specrum & type \\
\hline & $\operatorname{tamp}$ & lamp & & & & $\operatorname{lamp}$ & lamp & & & & lamp & lamp & & \\
\hline 4720.390 & & 2 & II & & 4666.735 & 200 & 10 & I & 7 & 4620.51 & & 2 & II & \\
\hline 4718.56 & 3000 & 500 & I & 3 & 4666.145 & 2 & 1 & II & & 4618.487 & 15 & & I & \\
\hline 4718.34 & 200 & 2 & I & & 4664.565 & 30 & & I & & 4617.900 & 5 & & I & \\
\hline 4717.375 & 30 & & I & & 4663.873 & 5 & & I & & 4617.46 & 1 & & I & \\
\hline 4715.672 & & 3 & II & & 4663.44 & 1 & & I & & 4615.947 & 300 & 15 & I & 1 \\
\hline 4715.16 & 6 & & I & & 4661.878 & 5 & & I & & 4615.740 & & 2 & II & \\
\hline 4714.70 & 700 & 40 & I & & 4660.906 & & 4 & II & & 4615.427 & 3 & & I & \\
\hline 4712.78 & 40 & 200 & II & 5 & 4660.68 & & $2 \mathrm{e}$ & III & & 4614.020 & 1 & 3 & II & \\
\hline 4712.73 & 2 & & I & & 4660.057 & 4 & & I & & 4611.90 & & 1 & II & \\
\hline 4709.96 & 15 & 1 & I & & 4659.910 & & 5 & II & & 4610.70 & & 1 & II & \\
\hline 4709.330 & 40 & 1 & I & & 4659.780 & 20 & & I & & 4610.172 & 600 & 30 & I & 3 \\
\hline 4706.81 & 2 & & I & & 4659.60 & & 1 & II & & 4609.600 & & 1 & II & \\
\hline 4706.700 & 2 & & I & & 4658.87 & $4 d$ & & I & & 4608.131 & & 15 & II & \\
\hline 4705.40 & & 4 & II & & 4658.746 & & 3 & II & & 4608.061 & 3 & & I & \\
\hline 4705.04 & & 7 & II & & 4658.443 & 2 & 2 & I,II & & 4607.004 & & $3 \mathrm{e}$ & III & \\
\hline 4704.886 & 300 & 20 & I & & 4657.43 & & 1 & II & & 4606.854 & 1 & & I & \\
\hline 4704.23 & & 1 & II & & 4656.971 & 3000 & 500 & I & 2 & 4602.354 & 2 & & I & \\
\hline 4703.012 & & 4 & II & & 4654.46 & 4 & & I & & 4600.300 & & 2 & II & \\
\hline 4702.982 & 10 & & I & & 4654.070 & 1 & 5 & II & & 4598.85 & & 2 & II & \\
\hline 4702.355 & 200 & 10 & I & & 4653.310 & 5 & & I & & 4598.361 & 300 & 800 & II & 5 \\
\hline 4700.77 & & $1 \mathrm{e}$ & III & & 4652.667 & & 5 & II & & 4597.980 & 5 & 30 & II & \\
\hline 4699.045 & & 6 & II & & 4652.279 & & 2 & II & & 4597.279 & 2 & 30 & II & \\
\hline 4698.62 & 10 & 40 & II & & 4651.67 & 500 & 20 & I & 3 & 4597.087 & & 1 & II & ? \\
\hline 4697.688 & & 2 & II & & 4650.99 & 3 & & I & & 4596.342 & & 7 & II & 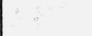 \\
\hline 4697.34 & & 3 & II & & 4650.05 & 1500 & 100 & I & 7 & 4595.556 & & 1 & II & \\
\hline 4696.842 & 1 & 15 & II & & 4649.756 & & 2 & II & & 4594.033 & 10 & & I & \\
\hline 4696.286 & 40 & 2 & I & & 4647.076 & & 2 & II & & 4593.375 & 30 & 5 & I & \\
\hline 4695.832 & 7 & & I & & 4646.91 & 7 & & I & & 4593.214. & & 4 & II & \\
\hline 4695.70 & 2 & & I & & 4646.532 & & $2 \mathrm{e}$ & III & & 4592.443 & & 10 & II & \\
\hline 4693.06 & 1 & & I & & 4645.05 & & 10 & II & & 4591.780 & 5 & & I & \\
\hline 4692.41 & & 2 & II & & 4644.54 & 3000 & 100 & I & 1 & 4591.333 & & 4 & III & \\
\hline 4691.982 & & 4 & II & & 4643.14 & & 5 & II & & 4590.834 & 2000 & 200 & I & 3 \\
\hline 4690.810 & 20 & 90 & II & & 4641.945 & 6 & 2 & I & & 4590.578 & 10 & & I & \\
\hline 4689.807 & 1 & 7 & II & & 4640.11 & & 15 & II & & 4589.211 & 1000 & 150 & I & 2 \\
\hline 4689.069 & 15 & & I & & 4639.14 & & $40 \mathrm{e}$ & III & & 4588.677 & & 1 & II & \\
\hline 4688.515 & 40 & 100 & II & 4 & 4639.04 & & 1 & II & & 4587.547 & & 2 & III & \\
\hline 4688.38 & 5 & 20 & II & & 4638.81 & 3 & & I & & 4587.075 & & 15 & II & \\
\hline 4687.593 & 1000 & 50 & I & 1 & 4637.88 & & $1 \mathrm{e}$ & III & & 4585.916 & 40 & 4 & I & 7 \\
\hline 4685.876 & & 6 & II & & 4637.341 & 3 & & I & & 4585.767 & & 10 & II & \\
\hline 4685.28 & 4 & & I & & 4637.226 & & 3 & II & & 4585.634 & 15 & & I & \\
\hline 4685.071 & & 3 & II & & 4635.01 & & 1 & II & & 4585.528 & & 2 & III & \\
\hline 4684.268 & 1500 & 80 & I & 7 & 4634.38 & 4 & & ! & & 4582.924 & 60 & 6 & I & \\
\hline 4683.810 & 200 & 1000 & II & 7 & 4634.014 & 20 & 200 & II & 7 & 4582.695 & 50 & 6 & I & \\
\hline 4681.666 & 10 & 1 & I & & 4633.406 & & $3 \mathrm{e}$ & III & & 4582.355 & $6000 \mathrm{R}$ & 600 & I & 3 \\
\hline 4681.514 & 3 & & I & & 4633.196 & 30 & 4 & I & & 4581.222 & 7 & & I & \\
\hline 4681.088 & & 3 & II & & 4631.09 & 2 & & I & & 4580.724 & 9 & 1 & I & \\
\hline 4680.136 & 6 & 1 & I & & 4630.614 & & 3 & II & & 4.580 .649 & 40 & 6 & I & \\
\hline 4679.625 & 15 & & I & & 4630.522 & 20 & & I & & 4579.536 & & 6 & II & \\
\hline 4678.171 & 2 & 6 & II & & 4629.833 & 200 & 3 & I & & 4578.620 & & 3 & II & \\
\hline 4677.432 & 90 & 1 & I & & 4629.75 & & 2 & II & & 4578.142 & & 7 & II & \\
\hline 4676.67 & 6 & & I & & 4628.861 & 2 & & I & & 4577.722 & 10 & & I & \\
\hline 4675.66 & 2 & 4 & II & & 4628.43 & 2 & & I & & 4576.209 & $10000 \mathrm{R}$ & 1000 & I & 1 \\
\hline 4675.20 & 1 & & I & & 4627.556 & 4. & & I & & 4574.800 & & 2 & II & \\
\hline 4674.644 & & 1 & II & & 4627.23 & 5 & & I & & 4572.740 & & 1 & II & \\
\hline 4674.493 & 1 & 3 & II & & 4626.054 & 2 & & I & & 4572.027 & & 1 & II & \\
\hline 4673.62 & 1 & & I & & 4625.47 & 2 & & I & & 4571.660 & & 3 & II & \\
\hline 4672.125 & 1 & & I & & 4624.93 & & 2 & II & & 4571.064 & & 1 & II & \\
\hline 4671.701 & 30 & 1 & I & & 4624.585 & & 4 & III & & 4570.081 & & 1 & II & \\
\hline 4671.386 & & 3 & II & & 4624.41 & 1200 & 50 & I & 2 & 4569.172 & & 2 & III & \\
\hline 4670.586 & 50 & 200 & II & 4 & 4623.91 & & 1 & II & & 4568.853 & 200 & 50 & I & 3 \\
\hline 4669.510 & 2 & & I & & 4623.060 & & 3 & II & & 4567.368 & 700 & 60 & I & 2 \\
\hline 4669.280 & & 4 & II & & 4621.83 & & 2 & II & & 4563.95 & 2000 & 200 & I & 1 \\
\hline 4667.05 & 1 & 1 & II & & 4621.80 & 3 & & & & 4563.680 & & 3 & UI & \\
\hline
\end{tabular}


TABLE 1.-Emission spectra of ytterbium-Continued

\begin{tabular}{|c|c|c|c|c|c|c|c|c|c|c|c|c|c|c|}
\hline \multirow{3}{*}{$\begin{array}{c}\text { Wave- } \\
\text { length } \\
\text { in air }\end{array}$} & \multicolumn{2}{|c|}{ Intensity } & \multirow{3}{*}{ Spectrum } & \multirow{3}{*}{$\begin{array}{c}\text { Zeeman } \\
\text { type }\end{array}$} & \multirow{3}{*}{$\begin{array}{c}\begin{array}{c}\text { Wave- } \\
\text { length } \\
\text { in air }\end{array} \\
\circ \\
\end{array}$} & \multicolumn{2}{|c|}{ Intensity } & \multirow{3}{*}{ Spectrum } & \multirow{3}{*}{$\begin{array}{c}\text { Zeeman } \\
\text { type }\end{array}$} & \multirow{3}{*}{$\begin{array}{c}\text { Wave- } \\
\text { length } \\
\text { in air }\end{array}$} & Inte & nsity & & \\
\hline & Meggers & Thompson & & & & Meggers & Thompson & & & & Meggers & Thompson & opecing & type \\
\hline & lamp & lamp & & & & lamp & lamp & & & & lamp & lamp & & \\
\hline 4562.440 & & 5 & II & & 4517.218 & & 2 & II & & 4463.965 & & 2 & II & \\
\hline 4561.533 & & 2 & III & & 4517.051 & & 1 & II & & 4463.855 & 1 & 8 & II & \\
\hline 4560.022 & & 3 & II & & 4516.094 & 1 & 2 & II & & 4463.493 & 1 & 9 & II & \\
\hline 4558.906 & & 3 & III & & 4515.636 & & 2 & II & & 4461.72 & 7 & & I & \\
\hline 4558.095 & 2 & & I & & 4515.161 & 200 & 800 & II & 4 & 4460.923 & 1 & 5 & II & \\
\hline 4557.936 & 20 & 150 & II & & 4514.846 & & 10 & II & & 4450.405 & & 1 & $\boldsymbol{N}$ & \\
\hline 4555.50 & & 2 & II & & 4513.990 & 6 & 20 & II & & 4459.181 & & 1 & II & \\
\hline 4555.47 & 3 & & I & & 4513.408 & 1000 & 80 & I & 3 & 4457.428 & & 2 & II & \\
\hline 4554.217 & & $5 \mathrm{e}$ & III & & 4512.909 & & 1 & II & & 4457.311 & & 1 & II & \\
\hline 4554.032 & 1 & 3 & II & & 4511.725 & & 3 & II & & 4456.994 & & 4 & II & \\
\hline 4553.576 & 500 & 1500 & II & 6 & 4510.744 & & 3 & II & & 4455.651 & 4 & 1 & I & \\
\hline 4552.619 & & 8 & II & & 4509.700 & & 3 & II & & 4452.663 & & 3 & II & \\
\hline 4552.351 & 6 & & I & & 4508.788 & 1 & 9 & II & & 4452.153 & & 1 & II & \\
\hline 4552.175 & & 2 & II & & 4506.95 & & $2 \mathrm{~d}$ & II & & 4451.930 & & 20 & II & \\
\hline 4551.792 & & $2 \mathrm{~d}$ & II & & 4506.045 & 1 & 4 & II & & 4451.11 & & $2 \mathrm{~d}$ & II & \\
\hline 4550.601 & & 6 & II & & 4505.900 & & 5 & II & & 4450.60 & & 1 & II & \\
\hline 4548.359 & 40 & 80 & II & & 4505.345 & & 3 & II & & 4444.860 & & 3 & II & \\
\hline 4547.960 & & 3 & II & & 4504.850 & & 2 & II & & 4444.108 & & 2 & III & \\
\hline 4547.788 & 10 & & I & & 4504.331 & 1 & 9 & II & & 4442.075 & & 3 & II & \\
\hline 4547.032 & 1 & 20 & II & & 4504.076 & & 3 & III & & 4441.484 & & 2 & II & \\
\hline 4546.866 & 30 & 1 & I & & 4503.636 & 600 & 50 & I & 3 & 4441.031 & 9 & 2 & I & \\
\hline 4545.770 & 8 & 1 & I & & 4503.463 & & 5 & II & & 4440.415 & & 3 & III & \\
\hline 4545.383 & & 1 & II & & 4503.21 & 150 & 5 & I & & 4440.011 & 4 & & I & \\
\hline 4545.216 & & 2 & II & & 4500.542 & & 3 & II & & 4439.19 & $7000 \mathrm{R}$ & 500 & I & 2 \\
\hline 4544.420 & 30 & 1 & I & & 4500.304 & & 1 & II & & 4438.142 & & 7 & II & \\
\hline 4543.879 & 2 & 40 & II & & 4499.621 & & 3 & II & & 4436.366 & 4 & & I & \\
\hline 4543.680 & 2 & & I & & 4499.462 & & 1 & II & & 4435.150 & & 5 & II & \\
\hline 4543.452 & & 4 & II & & 4498.098 & 1 & & I & & 4434.795 & 20 & 1 & I & \\
\hline 4543.302 & & 3 & II & & 4495.840 & 1 & & 1 & & 4434.178 & 40 & 100 & II & \\
\hline 4542.588 & $1 \mathrm{~h}$ & 5 & II & & 4495.520 & & 1 & II & & 4432.041 & 1 & & 1 & \\
\hline 4542.371 & & 1 & II & & 4493.965 & 50 & 150 & II & 6 & 4431.595 & & 1 & II & \\
\hline 4541.598 & & 2 & II & & 4493.244 & & 1 & II & & 4430.208 & 900 & 150 & I & 3 \\
\hline 4541.452 & 1 & & I & & 4492.120 & 4 & & I & & 4429.689 & & 2 & III & \\
\hline 4541.325 & 20 & 100 & II & 5 & 4491.422 & & 1 & II & & 4428.980 & 30 & 1 & I & \\
\hline 4540.046 & 8 & 2 & I & & $4490.240^{\circ}$ & & 1 & II & & 4428.782 & 700 & 100 & I & \\
\hline 4539.323 & 5 & 20 & II & & 4490.161 & 2 & & I & & 4428.153 & & $3 \mathrm{~h}$ & II & \\
\hline 4538.678 & 4 & & I & & 4488.282 & 1000 & 60 & I & 1 & 4428.02 & 3 & & I & \\
\hline 4538.027 & & 3 & II & & 4487.266 & 50 & 200 & II & 7 & 4427.387 & 600 & 200 & I & \\
\hline 4537.494 & & 2 & II & & 4487.03 & & $2 \mathrm{~h}$ & II & & 4423.129 & & 1 & II & \\
\hline 4536.805 & & 3 & II & & 4486.794 & & 1 & II & & 4422.150 & & 3 & II & \\
\hline 4536.313 & 2 & & I & & 4486.225 & & 1 & II & & 4422.070 & 1 & & I & \\
\hline 4535.718 & & 2 & II & & 4485.986 & & 1 & II & & 4419.616 & 3 & & I & \\
\hline 4534.250 & & 1 & II & & 4485.326 & & 6 & II & & 4419.495 & & 1 & II & \\
\hline 4533.506 & 1000 & 50 & I & 3 & 4485.040 & & 2 & II & & 4418.132 & & 3 & II & \\
\hline 4533.006 & 150 & 8 & I & & 4484.466 & 20 & 60 & II & & 4417.814 & & 1 & II & \\
\hline 4532.702 & 50 & 2 & I & & 4484.280 & 1 & 5 & II & & 4417.080 & & 3 & II & \\
\hline 4531.333 & 1500 & 200 & I & 1 & 4483.152 & 8 & 1 & I & & 4415.202 & 1 & & I & \\
\hline 4530.657 & & 3 & II & & 4482.422 & 1500 & 150 & I & 3 & 4414.548 & & 1 & II & \\
\hline 4530.23 & & 4 & III & & 4478.271 & 30 & 1 & I & & 4412.075 & & 2 & III & \\
\hline 4529.87 & 3000 & 400 & I & 1 & 4475.666 & & 2 & II & & 4411.095 & 500 & 30 & I & 3 \\
\hline 4528.882 & & 5 & II & & 4474.115 & & 3 & II & & 4410.740 & & $2 \mathrm{~h}$ & II & \\
\hline 4527.526 & & 2 & II & & 4473.884 & & 1 & II & & 4410.230 & 200 & 8 & I & \\
\hline 4525.535 & & 1 & II & & 4473.005 & 20 & 2 & I & & 4409.685 & & $2 \mathrm{~h}$ & II & \\
\hline 4525.108 & & 3 & II & & 4472.470 & 1500 & 300 & I & 7 & 4409.346 & 200 & 300 & II & 5 \\
\hline 4524.810 & & 2 & II & & 4472.146 & & 8 & II & & 4408.220 & & 1 & II & \\
\hline 4524.549 & 1 & 4 & II & & 4470.958 & & 1 & II & & 4407.650 & 1 & 4 & II & \\
\hline 4523.161 & & 2 & II & & 4469.566 & & 3 & II & & 4407.081 & 5 & 1 & I & \\
\hline 4521.338 & & 4 & III & & 4468.520 & 4 & & I & & 4405.496 & & 1 & II & \\
\hline 4520.918 & 2 & & I & & 4468.18 & 10 & 1 & I & & 4404.208 & & 1 & II & \\
\hline 4520.746 & & 8 & II & & 4467.642 & & 1 & II & & 4403.037 & & 1 & II & \\
\hline 4520.510 & 5 & 2 & I & & 4467.128 & 1 & 10 & II & & 4402.824 & & 5 & II & \\
\hline 4520.161 & 200 & 2 & I & & 4466.002 & & 7 & II & & 4402.605 & 40 & 2 & I & 7 \\
\hline 4517.584 & & 100 & III & & 4465.663 & & 2 & & & 4402.300 & 40 & 200 & II & \\
\hline
\end{tabular}


TABLE 1.-Emission spectra of ytterbium-Continued

\begin{tabular}{|c|c|c|c|c|c|c|c|c|c|c|c|c|c|c|}
\hline \multirow{3}{*}{\begin{tabular}{|c}
$\begin{array}{c}\text { Wave- } \\
\text { length } \\
\text { in air }\end{array}$ \\
$\AA$ \\
\end{tabular}} & \multicolumn{2}{|c|}{ Intensity } & \multirow{3}{*}{ Spectrum } & \multirow{3}{*}{$\begin{array}{c}\text { Zeeman } \\
\text { type }\end{array}$} & \multirow{3}{*}{$\begin{array}{c}\text { Wave- } \\
\text { length } \\
\text { in air }\end{array}$} & \multicolumn{2}{|c|}{ Intensity } & \multirow{3}{*}{ Spectrum } & \multirow{3}{*}{$\begin{array}{c}\text { Zeeman } \\
\text { type }\end{array}$} & \multirow{3}{*}{$\begin{array}{c}\begin{array}{c}\text { Wave- } \\
\text { length } \\
\text { in air }\end{array} \\
\AA \\
\AA\end{array}$} & Inte & nsity & & \\
\hline & Meggers & Thompson & & & & Meggers & Thompson & & & & Meggers & Thompson & | & type \\
\hline & lamp & lamp & & & & lamp & lamp & & & & lamp & lamp & & \\
\hline 4401.824 & & 1 & II & & 4363.71 & 3 & & I & & 4331.032 & & 5 & II & \\
\hline 4401.445 & 1 & 6 & II & & 4363.54 & & 1 & II & & 4330.488 & 20 & 1 & I & \\
\hline 4400.583 & & 1 & II & & 4363.280 & 6 & 90 & II & & 4329.818 & & 15 & II & \\
\hline 4400.332 & 50 & 2 & I & & 4362.333 & & 4 & II & & 4329.718 & 70 & 4 & I & 7 \\
\hline 4399.391 & & 1 & II & & 4362.156 & 1 & 6 & II & & 4329.654 & & 1 & II & \\
\hline 4398.96 & 2000 & 100 & I & 3 & 4361.868 & & 2 & II & & 4327.853 & & 9 & II & \\
\hline 4396.499 & 10 & 40 & II & & 4361.639 & 100 & $7 \mathrm{~h}$ & I & & 4327.238 & & 2 & II & \\
\hline 4396.254 & 100 & 5 & I & 7 & 4361.468 & 1 & 10 & II & 7 & 4326.637 & 1 & 15 & II & \\
\hline 4395.52 & 1 & & I & & 4360.663 & & 4 & II & & 4326.556 & & 9 & II & \\
\hline 4394.973 & 70 & 3 & I & & 4360.140 & 1 & 2 & II & & 4326.404 & 2000 & 200 & I & 1 \\
\hline 4394.177 & 25 & 2 & I & & 4359.958 & & 20 & II & & 4325.240 & 6 & 90 & II & \\
\hline 4393.688 & 2000 & $400 \mathrm{~h}$ & I & 7 & 4359.528 & 1000 & $150 \mathrm{~h}$ & I & 3 & 4325.033 & & 2 & II & \\
\hline 4392.825 & 50 & 100 & II & 6 & 4359.165 & 3 & 2 & I,II & & 4324.962 & & 1 & II & \\
\hline 4390.49 & & 1 & II & & 4356.676 & 500 & 40 & I & 2 & 4324.500 & & 7 & II & \\
\hline 4390.43 & & 1 & II & & 4356.468 & & 5 & III & & 4324.160 & & 1 & II & \\
\hline 4389.764 & 50 & 200 & II & & 4356.160 & & 3 & II & & 4323.630 & & 8 & II & \\
\hline 4389.15 & & 1 & III & & 4355.58 & & 1 & II & & 4323.554 & 1 & 5 & II & \\
\hline 4388.528 & & 8 & II & & 4354.191 & 3 & 10 & II & & 4322.851 & & 1 & II & \\
\hline 4387.46 & & 1 & II & & 4354.037 & 2 & 1 & I & & 4322.56 & 2 & 30 & II & \\
\hline 4387.32 & 30 & 2 & I & & 4353.570 & 500 & $90 \mathrm{~h} \mathrm{l}$ & I & & 4322.53 & 1 & 20 & II & \\
\hline 4387.144 & 1 & 10 & II & & 4352.948 & 2000 & 400 & I & 3 & 4322.230 & 200 & 2000 & II & 6 \\
\hline 4386.50 & 200 & 5 & I & & 4351.67 & 1 & & I & & 4320.995 & & 2 & III & \\
\hline 4385.97 & 2 & 20 & II & & 4351.07 & & 4 & III & & 4320.723 & & 2 & II & \\
\hline 4385.82 & & 1 & III & & 4350.801 & & $15 \mathrm{e}$ & III & & 4319.556 & 1 & 9 & II & \\
\hline 4385.66 & 3 & 10 & II & & 4350.44 & & 2 & III & & 4318.978 & & 1 & II & \\
\hline 4385.14 & 100 & 2 & I & & 4350.006 & 40 & 1 & I & & 4318.820 & & 1 & II & \\
\hline 4384.72 & & 2 & II & & 4349.952 & & 2 & II & & 4318.750 & & 2 & II & \\
\hline 4384.39 & & 4 & III & & 4349.470 & 2 & 1 & I & & 4318.459 & 2 & 10 & II & \\
\hline 4384.29 & 3 & & I & & 4348.807 & & 8 & III & & 4318.010 & & 3 & II & \\
\hline 4383.93 & & 10 & II & & 4348.087 & 3 & 15 & II & & 4317.751 & 1 & 15 & II & \\
\hline 4383.11 & & 1 & II & & 4347.66 & 3 & & I & & 4316.954 & 2000 & 8000 & II & 4 \\
\hline 4383.02 & & 2 & II & & 4347.358 & & 3 & II & & 4315.302 & & 3 & II & \\
\hline 4382.16 & & 1 & II & & 4347.223 & 10 & 20 & II & & 4315.15 & & 1 & II & \\
\hline 4382.08 & & 2 & II & & 4347.13 & & 1 & II & & 4314.65 & & 1 & III & \\
\hline 4381.87 & 2 & 1 & I & & 4347.013 & & 4 & III & & 4314.306 & & 2 & III & \\
\hline 4381.80 & & 20 & II & & 4346.00 & & 1 & II & & 4313.567 & 30 & 1 & I & \\
\hline 4380.30 & & 4 & III & & 4345.054 & & 3 & III & & 4313.156 & 70 & 4 & I & \\
\hline 4380.07 & & 10 & III & & 4344.894 & 1 & 20 & II & & 4312.991 & 60 & 3 & I & \\
\hline 4379.91 & & 8 & III & & 4344.762 & 300 & 20 & I & & 4312.356 & 600 & 40 & I & \\
\hline 4379.50 & 20 & 300 & II & & 4344.204 & 60 & 300 & II & 4 & 4311.86 & 2 & & I & \\
\hline 4379.452 & 200 & 20 & I & & 4343.845 & & 5 & II & & 4311.714 & & 5 & III & \\
\hline 4379.268 & 60 & 3 & I & & 4343.632 & 3 & & I & & 4310.230 & 1 & 10 & II & \\
\hline 4377.80 & & 1 & II & & 4343.110 & 2 & & I & & 4310.124 & & 20 & II & \\
\hline 4377.527 & 3000 & $300 \mathrm{~h} \mathrm{l}$ & I & & 4342.753 & & 3 & II & & 4309.886 & & 15 & II & \\
\hline 4376.851 & 1 & 40 & II & & 4342.151 & 6 & 80 & II & 5 & 4309.823 & 3000 & 300 & I & 7 \\
\hline 4376.649 & & 2 & II & & 4341.646 & & 10 & II & & 4309.308 & & 4 & II & \\
\hline 4376.456 & 2000 & 200 & I & 3 & 4340.673 & 2 & 7 & II & & 4309.085 & & 3 & III & \\
\hline 4375.814 & & 6 & II & & 4340.269 & & 1 & II & & 4308.73 & & 2 & III & \\
\hline 4374.861 & & 3 & II & & 4340.147 & 1 & 15 & II & & 4308.586 & & 7 & II & \\
\hline 4373.116 & 20 & 1 & I & & 4339.71 & 4 & & I & & 4307.820 & 300 & 10 & I & \\
\hline 4372.854 & & 1 & II & & 4339.38 & 30 & & I & & 4307.583 & & 4 & II & \\
\hline 4372.65 & & 2 & II & & 4339.082 & 150 & 1000 & II & 4 & 4307.29 & & 3 & II & \\
\hline 4372.560 & 2 & 30 & II & & 4337.599 & 80 & 20 & I & & 4306.494 & 2 & 80 & II & \\
\hline 4371.083 & & 3 & II & & 4337.235 & & 2 & II & & 4305.966 & 5000 & 2000 & I & 3 \\
\hline 4371.05 & & 1 & II & & 4336.430 & 1000 & $150 \mathrm{~h} \mathrm{l}$ & I & & 4305.484 & 150 & 20 & I & \\
\hline 4370.810 & 200 & 2000 & II & 6 & 4336.30 & & 1 & II & & 4305.369 & & 9 & III & \\
\hline 4368.89 & & 1 & II & & 4335.360 & 20 & 1 & I & & 4304.879 & & 5 & III & \\
\hline 4368.678 & 100 & $5 \mathrm{~h}$ & I & & 4334.914 & 3 & 4 & II & & 4304.512 & & 2 & II & \\
\hline 4368.520 & & 1 & II & & 4333.909 & 400 & 40 & I & & 4304.458 & & 3 & III & \\
\hline 4368.23 & 10 & & I & & 4332.809 & & 4 & II & & 4304.422 & & 2 & II & \\
\hline 4366.284 & 1000 & $200 \mathrm{~h} \mathrm{l}$ & I & & 4331.942 & & 2 & II & & 4304.01 & & 20 & III & \\
\hline 4365.948 & & $\begin{array}{l}2 \\
5\end{array}$ & II & & 4331.762 & & 3 & II & & $\begin{array}{l}4303.675 \\
4301.74\end{array}$ & & $\begin{array}{l}2 \\
3\end{array}$ & $\begin{array}{r}\text { II } \\
\text { III }\end{array}$ & \\
\hline 4364.056 & & 5 & II & & 4331.318 & & 1 & II & & 4301.74 & & 3 & IIII & \\
\hline
\end{tabular}


TABLE 1.-Emission spectra of ytterbium-Continued

\begin{tabular}{|c|c|c|c|c|c|c|c|c|c|c|c|c|c|c|}
\hline \multirow{3}{*}{$\begin{array}{c}\begin{array}{c}\text { Wave- } \\
\text { length } \\
\text { in air }\end{array} \\
\AA\end{array}$} & \multicolumn{2}{|c|}{ Intensity } & \multirow{3}{*}{ Spectrum } & \multirow{3}{*}{$\begin{array}{c}\text { Zeeman } \\
\text { type }\end{array}$} & \multirow{3}{*}{$\begin{array}{c}\text { Wave- } \\
\text { length } \\
\text { in air }\end{array}$} & \multicolumn{2}{|c|}{ Intensity } & \multirow{3}{*}{ Spectrum } & \multirow{3}{*}{$\begin{array}{c}\text { Zeeman } \\
\text { type }\end{array}$} & \multirow{3}{*}{$\begin{array}{c}\text { Wave- } \\
\text { length } \\
\text { in air }\end{array}$} & Inte & nsity & & \\
\hline & Meggers & Thompson & & & & Meggers & Thompson & & & & Meggers & Thompson & opectum & type \\
\hline & lamp & lamp & & & & lamp & lamp & & & & lamp & lamp & & \\
\hline 4301.496 & 5 & 15 & II & & 4267.590 & 50 & 100 & II & & 4242.425 & 4 & 60 & II & \\
\hline 4301.135 & & 40 & III & & 4267.132 & 500 & $30 \mathrm{~h} \mathrm{l}$ & I & & 4242.299 & & 4 & II & \\
\hline 4300.984 & 1000 & 80 & I & 7 & 4266.983 & 300 & 30 & I & & 4241.67 & 2 & 6 & II & \\
\hline 4300.736 & & 4 & II & & 4266.952 & 10 & 100 & II & & 4241.24 & & 2 & II & \\
\hline 4298.498 & & 2 & III & & 4266.70 & 20 & 1 & I & & 4241.159 & 1 & 15 & II & \\
\hline 4298.075 & & 1 & II & & 4266.156 & 3 & & I & & 4241.116 & & 5 & II & \\
\hline 4296.696 & & 3 & II & & 4266.115 & 2 & & I & & 4240.95 & 6 & & I & \\
\hline 4296.338 & & 3 & II & & 4265.976 & & 2 & II & & 4239.455 & & 1 & II & \\
\hline 4295.88 & 3 & & I & & 4265.498 & & 1 & II & & 4238.84 & & 1 & II & \\
\hline 4295.04 & 300 & & I & & 4265.144 & & 1 & III & & 4238.63 & 2 & 1 & I & \\
\hline 4295.023 & 500 & $50 \mathrm{hs}$ & I & & 4264.802 & 1 & 30 & II & & 4238.345 & & 1 & II & \\
\hline 4294.598 & & 3 & III & & 4264.520 & & 1 & II & & 4238.157 & 2 & 30 & II & \\
\hline 4294.473 & 30 & 2 & I & & 4264.282 & & 5 & II & & 4237.284 & & 3 & II & \\
\hline 4293.421 & & 8 & II & & 4264.029 & & 6 & II & & 4237.00 & 5 & & I & \\
\hline 4293.319 & & 3 & III & & 4262.826 & 2 & 5 & II & & 4235.69 & 7 & & I & \\
\hline 4292.99 & 3 & & I & & 4262.691 & & 6 & II & & 4235.565 & 1 & 20 & II & \\
\hline 4292.946 & & 5 & III & & 4262.273 & & 1 & II & & 4235.49 & 2 & & I & \\
\hline 4292.615 & 10 & 1 & I & & 4261.56 & & 8 & III & & 4235.012 & 1 & 20 & II & \\
\hline 4292.083 & 70 & 2 & I & & 4261.346 & 20 & 1 & I & & 4234.545 & 50. & 800 & II & 6 \\
\hline 4291.954 & 3 & 2 & I & & 4260.761 & & 2 & III & & 4233.777 & & 1 & I & \\
\hline 4290.357 & & 7 & II & & 4260.678 & & 7 & II & & 4233.445 & 150 & 8 & I & \\
\hline 4289.91 & & $i$ & II & & 4260.068 & & 3 & III & & 4232.427 & & 1 & II & \\
\hline 4289.64 & & 20 & III & & 4259.17 & & 1 & II & & 4231.972 & 10000 & 1000 & I & 3 \\
\hline 4289.605 & 1 & & I & & 4258.98 & & 4 & III & & 4231.648 & & 6 & II & \\
\hline 4288.840 & 80 & 7 & I & & 4258.743 & 200 & $20 \mathrm{hl}$ & I & & 4231.073 & & 15 & III & \\
\hline 4288.800 & & 9 & II & & 4258.194 & 2 & 20 & II & & 4230.184 & 100 & 400 & II & 4 \\
\hline 4286.839 & & 7 & II & & 4257.640 & 200 & 2000 & II & 4 & 4229.767 & & 4 & III & \\
\hline 4286.625 & & 4 & III & & 4257.550 & 40 & 10 & I & & 4229.681 & & 3 & II & \\
\hline 4285.878 & & 9 & II & & 4257.36 & 60 & 3 & I & & 4229.657 & 4 & & I & \\
\hline 4285.816 & & 1 & II & & 4257.198 & & 6 & II & & 4229.271 & 3 & 30 & II & \\
\hline 4285.258 & 1 & 9 & II & & 4256.756 & 1000 & 100 & I & & 4227.952 & 50 & 700 & II & 6 \\
\hline 4285.127 & 2 & 15 & II & & 4255.765 & 500 & 2000 & II & 4 & 4226.85 & & 2 & II & \\
\hline 4284.942 & 7 & 80 & II & & 4254.775 & 200 & 800 & II & 6 & 4226.595 & & 4 & II & \\
\hline 4284.170 & 2000 & $500 \mathrm{~h}$ & I & 7 & 4254.000 & & 9 & II & & 4226.523 & & 4 & III & \\
\hline 4283.782 & & 7 & III & & 4253.790 & & 1 & II & & 4226.273 & 1 & 20 & II & \\
\hline 4283.453 & & 1 & II & & 4253.488 & & 7 & III & & 4226.152 & 300 & $10 \mathrm{~h}$ & I & \\
\hline 4282.808 & & 10 & II & & 4253.363 & 3 & & I & & 4225.998 & & 8 & II & \\
\hline 4282.098 & 40 & 2 & I & & 4253.292 & 1 & & I & & 4225.548 & 10 & 30 & II & \\
\hline 4281.972 & 20 & 50 & II & & 4252.941 & 20 & 1 & I & & 4225.303 & & 1 & III & \\
\hline 4281.850 & 20 & 1 & I & & 4252.806 & & 3 & II & & 4224.196 & 20 & 150 & II & 4 \\
\hline 4281.584 & & 5 & II & & 4252.515 & 300 & 3000 & II & 4 & 4224.064 & & 1 & II & \\
\hline 4281.384 & & 2 & III & & 4252.040 & & 15 & II & & 4223.621 & 50 & 2 & I & \\
\hline 4280.636 & & 2 & II & & 4251.521 & 3000 & 200 & I & 3 & 4223.466 & & 2 & II & \\
\hline 4280.594 & & 1 & II & & 4250.21 & & 5 & II & & 4222.486 & & 10 & II & \\
\hline 4277.738 & 4000 & 1000 & I & 3 & 4249.861 & & 2 & II & & 4222.32 & 5 & & I & \\
\hline 4277.326 & & 8 & III & & 4249.81 & & 2 & II & & 4222.057 & & 4 & II & \\
\hline 4277.092 & & 2 & II & & 4249.748 & & 4 & II & & 4220.831 & & 10 & III & \\
\hline 4276.78 & & 1 & II & & 4248.12 & & 2 & II & & 4220.69 & & 4 & III & \\
\hline 4276.21 & & 2 & III & & 4247.885 & 40 & 300 & II & 6 & 4220.507 & 7 & 1 & I & \\
\hline 4275.694 & & 4 & III & & 4247.04 & & 2 & II & & 4220.260 & & 1 & II & \\
\hline 4275.66 & 5 & & I & & 4246.88 & 4 & & I & & 4219.706 & 500 & $20 \mathrm{~h}$ & I & \\
\hline 4275.63 & 5 & & I & & 4246.835 & & 10 & II & & 4219.646 & & 10 & II & \\
\hline 4275.48 & 2 & & I & & 4246.586 & 4 & 20 & II & & 4219.247 & 1000 & 200 & I & \\
\hline 4273.74 & 10 & 1 & I & & 4245.740 & 3 & 15 & II & & 4218.693 & 3000 & 600 & I & 7 \\
\hline 4273.340 & 20 & 150 & II & 5 & 4245.597 & & 2 & II & & 4218.565 & 1000 & 5000 & II & 4 \\
\hline 4272.647 & 400 & $40 \mathrm{~h} \mathrm{l}$ & I & & 4245.245 & 10 & 1 & I & & 4217.79 & & 2 & II & \\
\hline 4272.103 & 1000 & 40 & I & & 4244.73 & & 1 & II & & 4217.69 & & 2 & II & \\
\hline 4271.963 & & 2 & II & & 4244.51 & & 8 & II & & 4217.110 & & 4 & II & \\
\hline 4271.798 & 600 & $70 \mathrm{~h} \mathrm{l}$ & I & & 4244.21 & 6 & 80 & II & 5 & 4216.885 & 6 & 60 & II & \\
\hline 4270.515 & 4 & 60 & II & & 4244.054 & 6 & 15 & II & & 4216.766 & 20 & & I & \\
\hline 4268.986 & & 4 & III & & 4243.92 & 3 & & I & & 4216.704 & 60 & 300 & II & 4 \\
\hline 4268.882 & & 1 & II & & 4243.494 & & 9 & II & & 4216.569 & & 2 & II & \\
\hline 4268.39 & 1 & & I & & 4242.86 & 4 & & I & & 4216.417 & & 8 & II & \\
\hline
\end{tabular}


TABLE 1.-Emission spectra of ytterbium-Continued

\begin{tabular}{|c|c|c|c|c|c|c|c|c|c|c|c|c|c|c|}
\hline \multirow{3}{*}{$\begin{array}{c}\begin{array}{c}\text { Wave- } \\
\text { length } \\
\text { in air }\end{array} \\
\AA\end{array}$} & \multicolumn{2}{|c|}{ Intensity } & \multirow{3}{*}{ Spectrum } & \multirow{3}{*}{$\begin{array}{c}\text { Zeeman } \\
\text { type }\end{array}$} & \multirow{3}{*}{$\begin{array}{c}\text { Wave- } \\
\text { length } \\
\text { in air }\end{array}$} & \multicolumn{2}{|c|}{ Intensity } & \multirow{3}{*}{ Spectrum } & \multirow{3}{*}{$\begin{array}{c}\text { Zeeman } \\
\text { type }\end{array}$} & \multirow{3}{*}{$\begin{array}{c}\text { Wave- } \\
\text { length } \\
\text { in air }\end{array}$} & Inte & nsity & & \\
\hline & Meggers & Thompson & & & & Meggers & Thompson & & & & Meggers & Thompson & 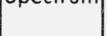 & type \\
\hline & lamp & lamp & & & & lamp & lamp & & & & lamp & lamp & & \\
\hline 4216.14 & & 1 & III & & 4190.297 & 200 & 2000 & II & 4 & 4157.755 & 100 & 200 & II & \\
\hline 4215.519 & 6 & 20 & II & & 4189.15 & 3 & & I & & 4157.292 & & 8 & III & \\
\hline 4215.485 & 2 & & I & & 4188.993 & 70 & $5 \mathrm{~h}$ & I & & 4156.575 & 1 & 4 & II & \\
\hline 4215.416 & & 2 & III & & 4188.723 & & 7 & II & & 4156.444 & & 1 & II & \\
\hline 4215.178 & & 2 & III & & 4188.371 & & 2 & II & & 4155.385 & 8 & 1 & I & \\
\hline 4214.942 & 2 & 15 & II. & & 4188.130 & 1 & 20 & II & & 4155.123 & 50 & $2 \mathrm{~h}$ & I & \\
\hline 4214.29 & 3 & & I & & 4187.73 & 1 & & I & & 4154.83 & 4 & & I & \\
\hline 4213.962 & $50 \mathrm{~d}$ & $20 \mathrm{~h}$ & I & & 4186.83 & 15 & 300 & II & 7 & 4154.484 & & 7 & III & \\
\hline 4213.856 & & 2 & II & & 4186.75 & 1 & & I & & 4153.88 & & 6 & II & \\
\hline 4213.641 & & 300 & III & & 4186.027 & & 2 & II & & 4153.758 & & 4 & II & \\
\hline 4213.407 & & 3 & II & & 4185.565 & 2 & 30 & II & 5 & 4153.197 & & 7 & II & \\
\hline 4213.106 & 10 & 1 & I & & 4184.635 & 1 & 20 & II & 7 & 4153.11 & & 15 & III & \\
\hline 4212.361 & & 6 & II & & 4184.289 & 2 & 1 & I & & 4152.79 & & 3 & II & \\
\hline 4211.957 & & 1 & II & & 4184.164 & & 3 & II & & 4152.38 & & 5 & III & \\
\hline 4211.82 & 2000 & 300 & I & 2 & 4183.905 & & 1 & II & & 4152.290 & 2 & & I & \\
\hline 4211.502 & & 2 & II & & 4183.402 & & 4 & III & & 4152.058 & 3 & & I & \\
\hline 4211.298 & & 4 & II & & 4183.129 & 5 & & I & & 4151.64 & & 7 & III & \\
\hline 4210.664 & 4 & 40 & II & 7 & 4183.06 & & 3 & III & & 4151.19 & 9 & 1 & I & \\
\hline 4210.48 & 5 & & I & & 4182.553 & & 2 & II & & 4150.04 & & 10 & III & \\
\hline 4210.299 & 200 & 20 & I & 7 & 4182.353 & & 2 & III & & 4149.066 & $20000 \mathrm{R}$ & 2000 & I & 7 \\
\hline 4210.225 & 15 & & I & & 4182.07 & 9 & 1 & I & & 4148.440 & & 8 & II & \\
\hline 4209.870 & & 5 & II & & 4180.809 & 10000 & 20000 & II & 4 & 4147.774 & 1 & 30 & II & \\
\hline 4209.342 & 8 & 1 & I & & 4179.95 & 20 & & I & & 4146.984 & & 2 & II & \\
\hline 4208.625 & & 4 & II & & 4179.084 & 10 & 80 & II & & 4146.870 & 80 & 5 & I & \\
\hline 4208.519 & & 1 & II & & 4178.585 & 4 & 6 & II & & 4146.764 & 1 & 30 & II & \\
\hline 4208.138 & & 6 & III & & 4178.225 & & 8 & II & & 4146.160 & & 3 & II & \\
\hline 4207.65 & & 1 & II & & 4177.81 & 4 & 80 & II & 4. & 4144.700 & 1 & 20 & II & \\
\hline 4207.32 & 6 & 1 & I & & 4177.40 & 2 & 50 & II & & 4144.273 & 1 & 15 & II & \\
\hline 4206.042 & & 1 & III & & 4176.204 & & 3 & II & & 4143.498 & 500 & $30 \mathrm{~h}$ & I & \\
\hline 4205.907 & 2 & & I & & 4175.928 & 10 & 90 & II & & 4143.070 & & 4 & II & \\
\hline 4205.785 & 80 & $6 \mathrm{~h}$ & I & & 4175.868 & 100 & 10 & I & & 4142.364 & 1 & 30 & II & \\
\hline 4205.608 & & 4 & II & & 4175.835 & & 30 & II & & 4141.457 & 2 & 60 & II & 5 \\
\hline 4204.195 & 300 & 5 & I & & 4175.602 & 2 & 30 & II & 4 & 4140.307 & 8 & 1 & I & \\
\hline 4204.058 & & 5 & II & & 4175.545 & 1 & 20 & II & & 4139.397 & 2 & & I & \\
\hline 4203.254 & & 2 & II & & 4174.56 & 3000 & 300 & I & 2 & 4139.332 & 10 & 30 & II & \\
\hline 4202.907 & 6 & 2 & I & & 4174.399 & & 6 & II & & 4139.051 & 500 & 20 & I & \\
\hline 4202.666 & & 2 & II & & 4174.259 & 80 & $4 \mathrm{~h}$ & I & & 4138.445 & & 4 & II & \\
\hline 4202.44 & 15 & $30 \mathrm{hl}$ & II & & 4173.134 & 9 & 1 & I & & 4138.166 & & 3 & II & \\
\hline 4201.991 & & 1 & II & & 4172.95 & & 60 & III & & 4138.13 & & 1 & II & \\
\hline 4201.864 & 3 & 1 & I & & 4172.422 & 200 & 20 & I & & 4138.11 & & 1 & II & \\
\hline 4201.460 & 2 & 20 & II & & 4172.15 & & 20 & II & 7 & 4137.985 & & 2 & II & \\
\hline 4201.291 & 200 & $8 \mathrm{~h}$ & I & & 4171.222 & 20 & 1 & I & & 4137.378 & & 4 & II & \\
\hline 4200.841 & & 5 & II & & 4170.106 & 200 & 2000 & II & 5 & 4136.930 & & 7 & II & \\
\hline 4200.72 & 60 & 1 & I & & 4169.499 & 2 & 8 & II & & 4136.872 & & 1 & II & \\
\hline 4199.939 & & 4 & II & & 4169.260 & 50 & $5 \mathrm{~h}$ & I & & 4136.35 & & 2 & III & \\
\hline 4199.713 & & 10 & II & & 4169.141 & 50 & 5 & I & & 4135.88 & & 3 & III & \\
\hline 4198.863 & & 1 & II & & 4167.37 & & 1 & III & & 4135.088 & 500 & 5000 & II & 6 \\
\hline 4198.738 & & 10 & III & & 4166.743 & 40 & 3 & I & & 4134.730 & 20 & 1 & I & \\
\hline 4198.18 & 2 & & I & & 4165.692 & & 2 & II & & 4134.476 & 20 & 80 & II & 6 \\
\hline 4197.876 & 400 & $100 \mathrm{~h}$ & I & & 4165.143 & & 6 & II & & 4132.722 & & 6 & III & \\
\hline 4196.632 & 1 & 9 & II & & 4165.08 & & 1 & III & & 4132.465 & & 4 & II & \\
\hline 4196.54 & 1 & & I & & 4164.660 & 5 & 50 & II & & 4132.165 & 15 & 2 & 1 & \\
\hline 4195.056 & 2 & & I & & 4164.587 & & 15 & II & & 4132.063 & & 2 & II & \\
\hline 4194.95 & & 100 & III & & 4163.770 & 3 & & I & & 4131.600 & 2 & 20 & II & \\
\hline 4194.39 & 2 & & I & & 4163.502 & 10 & 2 & I & & 4131.325 & & 3 & II & \\
\hline 4194.34 & & 30 & III & & 4162.717 & & 100 & III & & 4131.255 & 2 & & I & \\
\hline 4194.23 & 2 & & I & & 4162.023 & & 5 & II & & 4131.15 & & 4 & II & \\
\hline 4194.19 & 2 & & I & & 4161.572 & & 1 & II & & 4131.024 & 6 & 40 & II & \\
\hline 4193.807 & 2 & 10 & II & 6 & 4161.178 & & 7 & III & & 4130.878 & 1 & 4 & II & \\
\hline 4193.533 & & 4 & II & & 4160.299 & & 2 & II & & 4130.645 & 2 & & I & \\
\hline 4192.725 & & 3 & II & & 4160.243 & 4 & & I & & 4129.413 & & 1 & II & \\
\hline 4191.473 & & 2 & II & & 4160.090 & 3 & 40 & Id & & 4129.329 & & 2 & II & \\
\hline 4191.36 & 1 & & I & & 4159.008 & 6 & 1 & I & & 4129.157 & 2 & 30 & II & 4 \\
\hline
\end{tabular}


TABLE 1.-Emission spectra of ytterbium-Continued

\begin{tabular}{|c|c|c|c|c|c|c|c|c|c|c|c|c|c|c|}
\hline \multirow{3}{*}{$\begin{array}{c}\begin{array}{c}\text { Wave- } \\
\text { length } \\
\text { in air }\end{array} \\
\AA \\
\end{array}$} & \multicolumn{2}{|c|}{ Intensity } & \multirow{3}{*}{ Spectrum } & \multirow{3}{*}{$\begin{array}{c}\text { Zeeman } \\
\text { type }\end{array}$} & \multirow{3}{*}{$\begin{array}{c}\begin{array}{c}\text { Wave- } \\
\text { length } \\
\text { in air }\end{array} \\
\circ \\
\end{array}$} & \multicolumn{2}{|c|}{ Intensity } & \multirow{3}{*}{ Spectrum } & \multirow{3}{*}{$\begin{array}{c}\text { Zeeman } \\
\text { type }\end{array}$} & \multirow{3}{*}{$\begin{array}{c}\text { Wave- } \\
\text { length } \\
\text { in air }\end{array}$} & & ensity & & \\
\hline & Meggers & Thompson & & & & Meggers & Thompson & & & & Meggers & Thompson & opection & type \\
\hline & lamp & lamp & & & & lamp & lamp & & & & lamp & lamp & & \\
\hline 4128.934 & 100 & $4 \mathrm{~h}$ & 1 & & 4103.416 & 1 & 10 & II & & 4074.705 & 8 & 60 & II & 5 \\
\hline 4128.815 & 30 & 2 & I & & 4103.113 & & 2 & II & & 4074.528 & & 20 & III & \\
\hline 4.128 .115 & 4 & & I & & 4102.478 & 20 & 10 & II & & 4074.359 & 3 & & I & \\
\hline 4128.046 & & 3 & II & & 4102.264 & & 5 & II & & 4074.074 & & 1 & II & \\
\hline 4127.202 & & 4 & II & & 4101.335 & 30 & 3 & I & & 4073.846 & 8 & & I & \\
\hline 4127.170 & 1 & & I & & 4100.877 & & 1 & II & & 4073.288 & 1 & & I & \\
\hline 4126.992 & 20 & 30 & II & & 4100.160 & & 8 & II & & 4072.923 & 2 & & I & \\
\hline 4.126 .726 & 1 & 15 & II & & 4099.820 & & 2 & II & & 4071.442 & & 1 & II & \\
\hline 4126.030 & & 2 & II & & 4099.482 & & 3 & II & & 4071.334 & & 3 & II & \\
\hline 4125.955 & 3 & & I & & 4099.338 & 20 & 1 & I & & 4071.115 & 2 & & I & \\
\hline 4125.538 & 150 & $5 \mathrm{~h}$ & I & & 4098.920 & 4 & & I & & 4070.527 & 8 & 50 & II & \\
\hline 4125.185 & 20 & 1 & I & & 4098.848 & & 2 & II & & 4069.013 & & 4 & II & \\
\hline 4124.736 & 2 & 30 & II & & 4098.232 & & 20 & III & & 4068.50 & & 2 & II & \\
\hline 4124.564 & & 1 & II & & 4098.096 & & 2 & II & & 4068.229 & & 2 & II & \\
\hline 4123.75 & 6 & 1 & I & & 4097.885 & 100 & 600 & II & 5 & 4067.461 & 4 & & I & \\
\hline 4123.180 & 1 & 20 & II & & 4097.080 & 6 & 2 & I & & 4067.115 & 30 & 2 & I & \\
\hline 4122.853 & 50 & 500 & II & 4 & 4096.968 & & 1 & II & & 4067.031 & & 2 & II & \\
\hline 4122.274 & 4 & 1 & I & & 4096.785 & & 3 & II & & 4067.00 & 1 & & I & \\
\hline 4122.143 & 8 & 1 & I & & 4096.644 & 2 & 20 & II & & 4066.809 & & 15 & II & \\
\hline 4121.902 & 200 & 20 & I & & 4096.156 & 1 & 15 & II & & 4066.520 & & 2 & II & \\
\hline 4121.345 & & 3 & II & & 4094.351 & & 2 & II & & 4065.963 & $20 \mathrm{~h}$ & 1 & I & \\
\hline 4121.062 & & 15 & III & & 4093.634 & 2 & 20 & II & & 4064.757 & & 15 & II & \\
\hline 4120.071 & 1 & 40 & II & & 4092.959 & & 10 & II & & 4063.927 & 1 & 30 & II & \\
\hline 4119.812 & & 2 & II & & 4092.052 & 1 & 4 & II & & 4063.726 & 1000 & 150 & I & 2 \\
\hline 4119.464 & 200 & 1500 & II & 5 & 4091.53 & 6 & 100 & II & & 4063.455 & 3000 & 500 & I & 3 \\
\hline 4119.25 & 20 & 30 & II & & 4091.466 & 2 & 40 & II & & 4062.35 & & 1 & II & \\
\hline 4119.087 & 30 & 3 & I & & 4090.666 & & 20 & III & & 4062.100 & & 2 & II & \\
\hline 4119.046 & 3 & 60 & II & & 4090.594 & & 3 & II & & 4061.614 & & 7 & II & \\
\hline 4118.395 & & 3 & II & & 4089.908 & 20 & 4 & 1 & & 4061.382 & 10 & 1 & I & \\
\hline 4118.180 & 8 & 2 & I & & 4089.68 & $10000 \mathrm{R}$ & 3000 & I & 3 & 4060.180 & & 1 & II & \\
\hline 4118.07 & 10 & 1 & I & & 4088.633 & & 2 & II & & 4060.04 & & 1 & II & \\
\hline 4117.177 & 1 & 30 & II & & 4088.528 & & 1 & II & & 4059.893 & & 2 & II & \\
\hline 4116.89 & 1 & & I & & 4087.67 & 5 & 1 & I & & 4059.470 & 40 & 7 & I & 7 \\
\hline 4116.654 & & 4 & II & & 4087.480 & $30 \mathrm{~d}$ & $5 \mathrm{~d}$ & I & & 4058.625 & 60 & 6 & I & \\
\hline 4116.000 & 4 & & I & & 4087.343 & 20 & 3 & I & & 4058.404 & 150 & 15 & 1 & \\
\hline 4115.70 & 1 & & I & & 4086.61 & 7 & 100 & II & 7 & 4058.238 & & 1 & II & \\
\hline 4115.454 & & 9 & II & & 4087.492 & & 8 & III & & 4058.065 & 1 & 6 & II & \\
\hline 4114.694 & 1 & & I & & 4085.66 & 1 & 8 & II & & 4056.142 & 500 & 2000 & II & 4 \\
\hline 4114.591 & 2 & 30 & II & & 4085.05 & & 3 & II & & 4055.468 & & 3 & II & \\
\hline 4114.31 & & 1 & III & & 4084.845 & & 3 & II & & 4055.24 & 2 & & I & \\
\hline 4113.879 & 1 & 20 & II & & 4084.515 & & 1 & II & & 4054.588 & & 1 & II & \\
\hline 4113.805 & 2 & & I & & 4084.47 & 4 & & I & & 4053.547 & & 2 & II & \\
\hline 4113.707 & & 5 & II & & 4083.870 & & 1 & II & & 4053.235 & & $1 \mathrm{~h}$ & II & \\
\hline 4113.389 & 40 & $2 \mathrm{~h}$ & I & & 4083.648 & 15 & $4 \mathrm{~h}$ & I & & 4053.11 & & 10 & II & \\
\hline 4113.046 & 40 & 400 & II & 6 & 4083.346 & 4 & & I & & 4052.860 & & 20 & II & \\
\hline 4112.768 & & 3 & III & & 4082.992 & 500 & 20 & I & & 4052.283 & 5000 & 1000 & I & 1 \\
\hline 4111.966 & & 3 & III & & 4082.814 & & 4 & II & & 4052.072 & 1000 & 60 & I & 1 \\
\hline 4111.381 & & 6 & II & & 4082.64 & & 1 & II & & 4051.935 & 30 & 3 & 1 & \\
\hline 4111.07 & 8 & & I & & 4082.586 & 2 & & I & & 4051.339 & 20 & 60 & II & 4 \\
\hline 4.110 .448 & & 2 & II & & 4082.529 & & 1 & II & & 4050.083 & 20 & 150 & II & 4 \\
\hline 4110.168 & & 10 & II & & 4081.05 & & 1 & II & & 4049.982 & & 5 & II & \\
\hline 4109.79 & 40 & 2 & I & & 4079.991 & 2 & 40 & II & 6 & 4049.335 & & 7 & II & \\
\hline 4109.654 & & 400 & II & 4 & 4079.88 & & 6 & II & & 4048.683 & & 5 & II & \\
\hline 4109.574 & 3000 & 400 & I & 2 & 4079.542 & & 3 & II & & 4048.554 & & 2 & II & \\
\hline 4109.306 & 2 & 40 & II & & 4079.164 & 2 & 20 & II & & 4048.21 & & 1 & II & \\
\hline 4108.845 & 5 & 15 & II & & 4078.36 & 7 & 70 & II & 4 & 4048.090 & & 2 & II & \\
\hline 4108.542 & 8 & 1 & I & & 4078.000 & $10 \mathrm{~h}$ & & I & & 4047.38 & 20 & 200 & II & 5 \\
\hline 4108.399 & & 4 & III & & 4077.713 & 10 & 20 & II & & 4046.998 & & 2 & II & \\
\hline 4107.222 & 20 & $3 \mathrm{~h}$ & I & & 4077.276 & 200 & 2000 & II & 5 & 4046.56 & & 1 & II & \\
\hline 4106.284 & & 3 & II & & 4076.82 & & 1 & II & & 4046.52 & & 1 & II & \\
\hline 4106.070 & & 5 & II & & 4076.198 & & $3 \mathrm{~h}$ & II & & 4046.37 & 1 & & 1 & \\
\hline 4104.60 & 2 & & I & & 4076.09 & & $3 \mathrm{~h}$ & II & & 4046.297 & & 4 & II & \\
\hline 4104.05 & 4 & & I & & 4075.873 & & 3 & II & & 4045.605 & & 1 & II & \\
\hline
\end{tabular}


TABLE 1.-Emission spectra of ytterbium-Continued

\begin{tabular}{|c|c|c|c|c|c|c|c|c|c|c|c|c|c|c|}
\hline \multirow{3}{*}{$\begin{array}{l}\text { Wave- } \\
\text { length } \\
\text { in air } \\
\AA \\
\end{array}$} & \multicolumn{2}{|c|}{ Intensity } & \multirow{3}{*}{ Spectrum } & \multirow{3}{*}{$\begin{array}{c}\text { Zeeman } \\
\text { type }\end{array}$} & \multirow{3}{*}{$\begin{array}{l}\text { Wave- } \\
\text { length } \\
\text { in air }\end{array}$} & \multicolumn{2}{|c|}{ Intensity } & \multirow{3}{*}{ Spectrum } & \multirow{3}{*}{$\begin{array}{c}\text { Zeeman } \\
\text { type }\end{array}$} & \multirow{3}{*}{$\begin{array}{c}\text { Wave- } \\
\text { length } \\
\text { in air } \\
\AA \\
\end{array}$} & Inte & nsity & & \\
\hline & Meggers & Thompson & & & & Meggers & Thompson & & & & Meggers & Thompson & 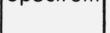 & type \\
\hline & lamp & lamp & & & & lamp & lamp & & & & lamp & lamp & & \\
\hline 4045.302 & 6 & 80 & II & & 4007.518 & & 20 & II & & 3976.148 & & 7 & II & \\
\hline 4044.657 & & 2 & II & & 4007.356 & 2000 & 500 & I & 3 & 3975.283 & 2000 & 100 & I & 2 \\
\hline 4044.464 & 3 & 1 & I & & 4006.962 & & 2 & II & & 3974.91 & & 1 & II & \\
\hline 4043.426 & & 4 & II & & 4006.828 & 15 & 2 & I & & 3974.30 & & 2 & II & \\
\hline 4043.05 & 20 & 300 & II & 5 & 4006.672 & & 1 & II & & 3973.87 & 10 & 3 & I & \\
\hline 4042.532 & 3 & 40 & II & & 4006.53 & & 2 & II & & 3972.91 & & 1 & II & \\
\hline 4042.328 & & 4 & II & & 4005.65 & $2 \mathrm{~h}$ & & I & & 3972.848 & & 3 & II & \\
\hline 4040.563 & 1 & 30 & II & 4 & 4005.619 & & 1 & II & & 3972.517 & & 4 & II & \\
\hline 4040.498 & & 8 & II & & 4005.218 & & 1 & II & & 3972.267 & & 3 & II & \\
\hline 4040.29 & & 1 & iI & & 4003.574 & & 1 & II & & 3972.18 & 3 & & I & \\
\hline 4040.08 & 60 & 1000 & II & 5 & 4003.224 & & 2 & II & & 3972.08 & & 1 & II & \\
\hline 4039.938 & 15 & 1 & I & & 4002.798 & & 2 & II & & 3972.01 & 7 & & I & \\
\hline 4039.613 & & 4 & II & & 4002.68 & & 1 & II & & 3971.739 & & 2 & II & \\
\hline 4039.140 & & 3 & II & & 4002.326 & & 10 & II & & 3971.014 & & $2 \mathrm{~h}$ & II & \\
\hline 4038.97 & 2 & 1 & I & & 4002.04 & $3 \mathrm{~h}$ & & I & & 3969.89 & 4 & 1 & I & \\
\hline 4038.94 & 2 & & I & & 4002.002 & & 1 & II & & 3969.519 & & 1 & II & \\
\hline 4038.187 & & 6 & II & & 4000.808 & 5 & 150 & II & 7 & 3969.461 & & 1 & II & \\
\hline 4037.916 & & 9 & II & & 4000.46 & 10 & & I & & 3969.082 & & 3 & II & \\
\hline 4037.822 & 20 & 50 & II & 7 & 4000.212 & 4 & 1 & I & & 3968.79 & & 20 & II & \\
\hline 4036.772 & 20 & 15 & I,II & & 3999.777 & 2 & 40 & II & & 3968.68 & & 1 & II & \\
\hline 4036.550 & 1 & 15 & II & & 3998.879 & 2 & 1 & I & & 3968.024 & 10 & 100 & II & 5 \\
\hline 4035.855 & 5 & 3 & I & & 3998.430 & & 7 & II & & 3966.062 & & 2 & II & \\
\hline 4035.51 & 5 & 1 & I & & 3998.38 & & 1 & II & & 3965.416 & & 1 & III & \\
\hline 4035.315 & 9 & 90 & II & 6 & 3998.133 & 3 & & I & & 3964.584 & & 1 & II & \\
\hline 4034.438 & & 6 & II & & 3997.667 & & 10 & III & & 3964.459 & 1 & 20 & II & 4 \\
\hline 4033.695 & & 4 & II & & 3997.244 & & 4 & II & & 3964.364 & & 1 & III & \\
\hline 4033.029 & & 10 & III & & 3995.859 & & 1 & II & & 3963.301 & & 2 & II & \\
\hline 4032.869 & 10 & 100 & II & 5 & 3995.432 & 1 & 20 & II & 7 & 3963.07 & 5 & & I & \\
\hline 4032.756 & & $2 \mathrm{~h}$ & II & & 3994.563 & & 2 & II & & 3962.457 & & 4 & II & \\
\hline 4032.441 & & 2 & II & & 3994.300 & & 1 & II & & 3961.98 & 70 & 6 & I & 1 \\
\hline 4030.712 & & 1 & II & & 3993.753 & 100 & 10 & I & 3 & 3961.832 & & 4 & II & \\
\hline 4029.844 & 2 & $60 \mathrm{~d}$ & II & & 3992.826 & & 1 & II & & 3961.731 & 5 & 1 & I & \\
\hline 4028.138 & & 2000 & III & 2 & 3992.09 & & 8 & II & & 3961.503 & 5 & 1 & I & \\
\hline 4027.580 & & 10 & II & & 3991.945 & & 4 & III & & 3961.288 & & 1 & II & \\
\hline 4027.19 & & 1 & II & & 3991.74 & & 10 & III & & 3961.104 & & 2 & II & \\
\hline 4026.922 & & 2 & II & & 3991.09 & & 1 & II & & 3960.866 & & 2 & II & \\
\hline 4025.740 & & 2 & II & & 3990.885 & $10000 \mathrm{R}$ & 1000 & I & 3 & 3960.34 & & 1 & II & \\
\hline 4024.375 & & 4 & II & & 3990.603 & & 2 & II & & 3960.27 & & 1 & II & \\
\hline 4023.987 & 6 & 30 & II & 5 & 3990.473 & & 2 & II & & 3959.730 & & 1 & II & \\
\hline 4022.712 & 3 & 40 & II & & 3990.280 & & 1 & II & & 3959.060 & 1 & 9 & II & \\
\hline 4021.956 & & 1 & II & & 3989.717 & & 6 & II & & 3958.818 & & 2 & II & \\
\hline 4021.56 & 2 & 1 & I & & 3988.666 & & 70 & II & & 3958.376 & & 3 & II & \\
\hline 4020.310 & & 2 & II & & 3987.99 & $50000 \mathrm{~A}$ & 30000 & I & 7 & 3957.270 & 6 & 2 & I & \\
\hline 4019.852 & 1 & 10 & II & & 3986.962 & & 5 & II & & 3957.05 & 5 & & I & \\
\hline 4019.35 & 6 & 150 & II & 4 & 3986.830 & & 5 & II & & 3954.266 & & 4 & II & \\
\hline 4018.966 & 30 & 3 & I & & 3986.179 & & 10 & II & & 3953.546 & & 1 & II & \\
\hline 4018.264 & & 2 & II & & 3985.564 & & 100 & III & & 3951.08 & & 10 & II & \\
\hline 4018.134 & & 2 & II & & 3985.125 & & 1 & II & & 3949.91 & 10 & 100 & II & 6 \\
\hline 4017.94 & & 1 & II & & 3984.765 & & 3 & II & & 3949.58 & 100 & 200 & II & 5 \\
\hline 4017.060 & & 3 & II & & 3983.748 & & 1 & II & & 3949.307 & & 2 & III & \\
\hline 4016.976 & & 9 & II & & 3983.314 & 2 & 60 & II & 4 & 3948.64 & & 4 & II & \\
\hline 4015.296 & & 4 & II & & 3983.179 & & 1 & II & & 3948.18 & 20 & 100 & II & 5 \\
\hline 4015.004 & & 1 & II & & 3982.009 & 2 & 20 & II & 4 & 3946.94 & 20 & 150 & II & 4 \\
\hline 4013.579 & 1 & 15 & II & & 3981.574 & & 1 & II & & 3945.577 & & 2 & II & \\
\hline 4013.464 & & 3 & II & & 3981.160 & & 5 & II & & 3944.55 & & 1 & II & \\
\hline 4012.704 & & 1 & II & & 3980.78 & & 1 & II & & 3944.509 & & 1 & II & \\
\hline 4012.204 & & 1 & II & & 3980.318 & & 10 & II & & 3943.692 & & 2 & II & \\
\hline 4010.84 & & 2 & II & & 3979.710 & 10 & 2 & I & & 3943.29 & 4 & 1 & I & \\
\hline 4010.825 & 3 & & I & & 3979.095 & 60 & 4 & I & & 3942.72 & & 6 & II & \\
\hline 4009.237 & & 20 & II & & 3978.543 & 2 & 10 & II & & 3939.780 & 10 & 1 & I & \\
\hline 4008.94 & 4 & 1 & II & & 3977.342 & 2 & 20 & II & & 3939.517 & 1 & 20 & II & \\
\hline 4008.775 & & 3 & II & & 3977.113 & & 1 & II & & 3938.53 & 40 & 400 & II & \\
\hline 4007.962 & 10 & 5 & I & & 3976.527 & & 1 & II & & 3938.25 & 40 & 500 & II & 6 \\
\hline
\end{tabular}


TABLE 1.-Emission spectra of ytterbium-Continued

\begin{tabular}{|c|c|c|c|c|c|c|c|c|c|c|c|c|c|c|}
\hline \multirow{3}{*}{$\begin{array}{l}\text { Wave- } \\
\text { length } \\
\text { in air }\end{array}$} & \multicolumn{2}{|c|}{ Intensity } & \multirow{3}{*}{ Spectrum } & \multirow{3}{*}{$\begin{array}{c}\text { Zeeman } \\
\text { type }\end{array}$} & \multirow{3}{*}{$\begin{array}{l}\text { Wave- } \\
\text { length } \\
\text { in air } \\
\circ \\
\text { A }\end{array}$} & \multicolumn{2}{|c|}{ Intensity } & \multirow{3}{*}{ Spectrum } & \multirow{3}{*}{$\begin{array}{c}\text { Zeeman } \\
\text { type }\end{array}$} & \multirow{3}{*}{$\begin{array}{l}\text { Wave- } \\
\text { length } \\
\text { in air } \\
\circ \\
\end{array}$} & Inte & nsity & & \\
\hline & Meggers & Thompson & & & & Meggers & Thompson & & & & Meggers & Thompson & Jpectrom & type \\
\hline & lamp & lamp & & & & lamp & lamp & & & & lamp & lamp & & \\
\hline 3937.984 & & 1 & II & & 3904.447 & & 1 & II & & 3872.338 & & 8 & II & \\
\hline 3936.596 & & 2 & II & & 3903.74 & & $1 \mathrm{~h}$ & II & & 3871.946 & & 1 & II & \\
\hline 3936.385 & & 5 & II & & 3903.397 & & 4 & II & & 3870.447 & & 2 & II & \\
\hline 3935.086 & & 3 & II & & 3903.288 & & 3 & II & & 3870.197 & & $2 \mathrm{~h}$ & II & \\
\hline 3934.30 & 4 & 100 & II & & 3902.814 & & 5 & II & & 3868.907 & 4 & 1 & I & \\
\hline 3934.182 & $3000 \mathrm{R}$ & 200 & I & & 3902.43 & & $3 \mathrm{~h}$ & II & & 3868.838 & & 4 & II & \\
\hline 3933.87 & & 2 & II & & 3902.386 & $10 \mathrm{~h}$ & 4 & I & & 3868.602 & 1 & 9 & II & \\
\hline 3933.25 & & $2 \mathrm{~h}$ & II & & 3902.109 & & 5 & II & & 3868.570 & 1 & 20 & II & \\
\hline 3931.90 & 10 & 100 & II & 6 & 3902.042 & 1 & 20 & II & & 3867.75 & & 1 & II & \\
\hline 3931.634 & & 2 & II & & 3901.44 & & 1 & II & & 3867.675 & & 3 & II & \\
\hline 3931.44 & & 1 & II & & 3901.06 & 10 & 1 & I & & 3867.48 & & $5 \mathrm{~h}$ & II & \\
\hline 3931.273 & 4 & & I & & 3900.85 & $4000 \mathrm{R}$ & 500 & I & 3 & 3867.378 & & 1 & II & \\
\hline 3931.227 & & 500 & III & & 3899.245 & & 3 & II & & 3866.862 & & 1 & II & \\
\hline 3930.900 & & 1 & II & & 3899.07 & 4 & 80 & II & 7 & 3865.95 & $3 \mathrm{~h}$ & & I & \\
\hline 3930.004 & & 2 & II & & 3898.450 & & 1 & II & & 3865.43 & 1 & & 1 & \\
\hline 3929.885 & & 1 & II & & 3897.946 & & 8 & II & & 3865.351 & & 1 & II & \\
\hline 3929.30 & 1 & & I & & 3897.555 & 10 & 2 & I & & 3865.255 & & 3 & II & \\
\hline 3929.053 & & 6 & II & & 3897.34 & & $2 \mathrm{~d}$ & II & & 3865.14 & 1 & & I & \\
\hline 3928.068 & & 10 & II & & 3897.060 & & 1 & II & & 3864.33 & $20 \mathrm{~h}$ & & 1 & \\
\hline 3927.60 & & 1 & II & & 3896.966 & & 4 & II & & 3863.784 & & 2 & II & \\
\hline 3926.962 & & 1 & II & & 3896.742 & 20 & 3 & I & & 3863.455 & 200 & 800 & II & 5 \\
\hline 3926.724 & 30 & 3 & I & & 3896.55 & & 150 & III & & 3863.046 & 10 & 100 & II & \\
\hline 3926.651 & & 2 & II & & 3895.884 & & 2 & II & & 3862.59 & 2 & & I & \\
\hline 3924.656 & & 2 & II & & 3895.758 & 1 & 30 & II & & 3862.511 & 1 & 20 & II & 5 \\
\hline 3924.527 & 10 & 2 & 1 & & 3893.740 & & 2 & II & & 3862.46 & 1 & & I & \\
\hline 3923.230 & & 4 & II & & 3892.285 & & 1 & II & & 3862.18 & 10 & 1 & I & \\
\hline 3922.443 & 1 & 15 & II & & 3891.696 & & 1 & II & & 3861.792 & & 3 & II & \\
\hline 3921.201 & & 2 & II & & 3890.850 & 2 & 50 & II & 4 & 3861.730 & & 2 & II & \\
\hline 3920.745 & & 4 & II & & 3890.767 & 20 & 3 & I & & 3861.566 & & 1 & II & \\
\hline 3920.602 & & $2 \mathrm{~h}$ & II & & 3889.664 & & 2 & II & & 3861.467 & & 1 & II & \\
\hline 3919.980 & 1 & 15 & II & & 3887.826 & & 10 & II & & 3860.975 & 2 & & I & \\
\hline 3918.804 & & 9 & II & & 3887.50 & & 1 & II & & 3860.917 & & 1 & II & \\
\hline 3917.685 & & 3 & II & & 3887.290 & 20 & 300 & II & 4 & 3860.815 & 10 & & I & \\
\hline 3917.473 & & 5 & II & & 3887.166 & & 20 & III & & 3860.64 & 3 & 1 & I & \\
\hline 3916.892 & & 2 & II & & 3886.63 & & 1 & II & & 3859.887 & & 2 & II & \\
\hline 3916.783 & 2 & 15 & II & & 3886.378 & & 4 & II & & 3859.35 & & $2 \mathrm{~h}$ & II & \\
\hline 3916.702 & & 1 & II & & 3885.647 & & 1 & II & & 3858.555 & 10 & 100 & II & 4 \\
\hline 3916.425 & & 10 & II & & 3885.470 & & 1 & II & & 3858.434 & & 1 & II & \\
\hline 3916.316 & & 1 & II & & 3885.195 & & 4 & II & & 3858.07 & & 9 & II & \\
\hline 3916.268 & & 1 & II & & 3884.833 & & 1 & II & & 3857.860 & & 2 & II & \\
\hline 3915.427 & & 10 & II & & 3883.638 & & 1 & II & & 3857.586 & 10 & 20 & II & \\
\hline 3914.690 & & 3 & II & & 3883.105 & & 1 & II & & 3856.514 & & 3 & II & \\
\hline 3913.923 & & 7 & II & & 3882.583 & & 10 & III & & 3856.009 & & 2 & III & \\
\hline 3913.729 & & 7 & II & & 3882.068 & 40 & 4 & I & & 3856.00 & 3 & & I & \\
\hline 3913.347 & 30 & 200 & II & 4 & 3881.738 & & 3 & II & & 3855.883 & 50 & 8 & I & \\
\hline 3913.234 & & 20 & III & & 3880.684 & & 1 & II & & 3854.91 & 4 & 30 & II & \\
\hline 3912.746 & & 15 & III & & 3880.332 & & 2 & II & & 3854.806 & 20 & 100 & II & 5 \\
\hline 3911.272 & 600 & 60 & I & 3 & 3880.197 & 5 & 70 & II & 4 & 3854.116 & 2 & & I & \\
\hline 3910.911 & & 6 & II & & 3880.044 & & 1 & II & & 3854.09 & 2 & & I & \\
\hline 3910.653 & 20 & 4 & I & & 3879.985 & & 20 & III & & 3853.731 & & 3 & II & \\
\hline 3910.429 & & 2 & II & & 3879.140 & & 7 & II & & 3853.238 & 1 & 2 & II & \\
\hline 3910.364 & & 4 & II & & 3878.023 & & 4 & II & & 3852.562 & & 10 & II & \\
\hline 3909.98 & & 1 & II & & 3877.06 & 4 & 60 & II & & 3852.160 & 4 & 40 & II & \\
\hline 3909.186 & & 1 & II & & 3876.902 & & 1 & II & & 3851.80 & & 2 & II & \\
\hline 3908.020 & & 5 & II & & 3876.776 & 1 & 20 & II & 5 & 3851.65 & 1 & & I & \\
\hline 3907.355 & 2 & 40 & II & 4 & 3876.33 & & $2 \mathrm{~h}$ & II & & 3851.36 & 5 & & I & \\
\hline 3906.960 & 4 & 30 & II & 6 & 3875.76 & 1 & & I & & 3851.242 & & 1 & II & \\
\hline 3906.64 & & 3 & II & & 3874.610 & & $3 \mathrm{~h}$ & II & & 3851.090 & & 2 & II & \\
\hline 3906.196 & 4 & 30 & II & & 3874.066 & & 10 & II & & 3850.98 & 7 & & I & \\
\hline 3905.832 & 20 & 200 & II & 6 & 3873.497 & & 4 & II & & 3850.366 & & 4 & II & \\
\hline 3905.51 & 5 & 1 & I & & 3873.246 & 5 & 1 & I & & 3850.32 & & 2 & II & \\
\hline 3904.812 & 100 & 1500 & II & 5 & 3872.852 & $5000 \mathrm{R}$ & 1000 & I & 7 & 3849.54 & 1 & & I & \\
\hline 3904.537 & & 2 & II & & 3872.555 & & 5 & II & & 3848.792 & 5 & 1 & I & \\
\hline
\end{tabular}


TABLE 1.-Emission spectra of ytterbium-Continued

\begin{tabular}{|c|c|c|c|c|c|c|c|c|c|c|c|c|c|c|}
\hline \multirow{3}{*}{$\begin{array}{c}\begin{array}{c}\text { Wave- } \\
\text { length } \\
\text { in air }\end{array} \\
\AA\end{array}$} & \multicolumn{2}{|c|}{ Intensity } & \multirow{3}{*}{ Spectrum } & \multirow{3}{*}{$\begin{array}{c}\text { Zeeman } \\
\text { type }\end{array}$} & \multirow{3}{*}{$\begin{array}{l}\text { Wave- } \\
\text { length } \\
\text { in air }\end{array}$} & \multicolumn{2}{|c|}{ Intensity } & \multirow{3}{*}{ Spectrum } & \multirow{3}{*}{$\begin{array}{c}\text { Zeeman } \\
\text { type }\end{array}$} & \multirow{3}{*}{$\begin{array}{c}\text { Wave- } \\
\text { length } \\
\text { in air }\end{array}$} & & ensity & & \\
\hline & Meggers & Thompson & & & & Meggers & Thompson & & & & Meggers & Thompson & opeciom & type \\
\hline & lamp & lamp & & & & lamp & lamp & & & & lamp & Iamp & & \\
\hline 3847.473 & 30 & 200 & II & 4 & 3819.916 & 1 & 15 & II & & 3789.37 & & 1 & II & \\
\hline 3846.237 & & 4 & II & & 3818.365 & & 1 & II & & 3788.267 & & 4 & II & \\
\hline 3846.186 & 1 & 15 & II & & 3817.777 & 3 & 20 & II & 5 & 3787.418 & 1 & 5 & II & \\
\hline 3845.79 & 1 & & I & & 3817.624 & & 7 & II & & 3787.080 & 1 & 10 & II & \\
\hline 3845.64 & 3 & & I & & 3817.09 & 1 & & I & & 3786.465 & & 1 & II & \\
\hline 3845.402 & & 4 & II & & 3816.345 & 15 & 150 & II & 6 & 3785.960 & & 1 & II & \\
\hline 3845.35 & 2 & & I & & 3816.189 & 30 & 300 & II & 4 & 3785.666 & 1 & 20 & II & 7 \\
\hline 3844.988 & 2 & 6 & II & & 3815.640 & & 1 & II & & 3785.330 & 6 & 80 & II & 4 \\
\hline 3844.349 & 1 & 15 & II & & 3815.203 & 200 & 10 & I & & 3784.512 & & 1 & II & \\
\hline 3843.811 & & 6 & II & & 3814.310 & & 10 & II & & 3784.384 & 7 & 100 & II & 6 \\
\hline 3843.420 & 5 & 80 & II & 6 & 3814.215 & 15 & 200 & II & 4 & 3784.144 & 1 & 10 & II & \\
\hline 3843.30 & 2 & & I & & 3814.084 & & 4 & II & & 3783.914 & & 1 & II & \\
\hline 3843.032 & 1 & 20 & II & & 3813.665 & & 3 & II & & 3782.80 & 1 & & I & \\
\hline 3842.653 & & 1 & II & & 3811.584 & & 2 & II & & 3782.736 & & 4 & II & \\
\hline 3841.950 & 1 & 9 & II & & 3809.04 & 5 & 30 & II & & 3782.540 & 70 & 700 & II & 5 \\
\hline 3841.296 & 1 & 10 & II & & 3808.809 & 10 & 1 & I & & 3781.684 & & 8 & II & \\
\hline 3840.70 & & 1 & II & & 3808.544 & & 1 & II & & 3781.415 & 1 & & I & \\
\hline 3840.341 & 3 & 70 & II & 7 & 3808.460 & 1 & 2 & II & & 3781.268 & & 1 & II & \\
\hline 3840.297 & 2 & 40 & II & & 3808.35 & & 2 & II & & 3781.046 & & 3 & II & \\
\hline 3840.013 & & 2 & II & & 3808.315 & & 2 & II & & 3780.605 & & 1 & II & \\
\hline 3839.907 & 1500 & 80 & I & 1 & 3807.574 & 50 & 500 & II & & 3780.217 & 2 & 20 & II & \\
\hline 3839.469 & 30 & 300 & II & 5 & 3807.545 & 100 & 1000 & II & 4 & 3779.838 & & 1 & II & \\
\hline 3839.382 & 1 & 20 & II & & 3806.996 & & 3 & II & & 3779.472 & & 2 & II & \\
\hline 3838.900 & 10 & 100 & II & 4 & 3806.869 & & 1 & II & & 3779.286 & 20 & 150 & II & 6 \\
\hline 3838.67 & & 2 & II & & 3806.818 & & 2 & II & & 3779.184 & 1 & 15 & II & \\
\hline 3838.287 & 80 & 4 & I & 2 & 3806.73 & 2 & & I & & 3778.800 & & 2 & II & \\
\hline 3838.123 & & 3 & II & & 3806.188 & 2 & 20 & II & & 3778.452 & & 3 & II & \\
\hline 3838.02 & 7 & 1 & I & & 3806.011 & & 1 & II & & 3777.906 & 1 & 20 & II & \\
\hline 3837.57 & 5 & 1 & I & & 3804.99 & & 1 & II & & 3777.534 & 2 & 30 & II & 6 \\
\hline 3837.411 & & 4 & II & & 3804.431 & 4 & 40 & II & 5 & 3776.520 & 3 & 40 & II & 5 \\
\hline 3836.677 & & 2 & II & & 3804.35 & & 2 & II & & 3776.362 & & 1 & II & \\
\hline 3836.541 & & 1 & II & & 3803.680 & & 1 & II & & 3775.906 & & 2 & II & \\
\hline 3836.46 & 5 & 1 & I & & 3802.47 & & 1 & II & & 3774.956 & & 2 & II & \\
\hline 3835.952 & & 2 & II & & 3802.28 & & 1 & II & & 3774.323 & 700 & 40 & I & 2 \\
\hline 3835.872 & & 1 & II & & 3801.752 & 1 & 20 & II & & 3773.625 & & 1 & II & \\
\hline 3835.495 & & 3 & II & & 3801.386 & 2 & & I & & 3773.428 & 200 & 600 & II & 6 \\
\hline 3834.35 & 1 & & I & & 3801.263 & 10 & 1 & I & & 3772.517 & 20 & 100 & II & 5 \\
\hline 3833.884 & & 9 & II & & 3801.026 & & $3 \mathrm{~h}$ & II & & 3772.294 & 3 & 40 & II & 5 \\
\hline 3832.701 & & 4 & II & & 3800.622 & 20 & 80 & II & 5 & 3771.768 & & 3 & III & \\
\hline 3832.664 & & 1 & II & & 3799.054 & & 1 & II & & 3771.596 & & 2 & II & \\
\hline 3832.180 & & 3 & II & & 3798.960 & 4 & 20 & II & & 3771.350 & & 3 & II & \\
\hline 3832.108 & & 3 & II & & 3798.854 & & 1 & II & & 3771.155 & 1 & 10 & II & \\
\hline 3831.610 & 3 & 20 & II & 7 & 3798.402 & 4000 & 400 & I & 2 & 3770.814 & & 1 & II & \\
\hline 3831.288 & & 1 & II & & 3798.162 & 3000 & 300 & I & 1 & 3770.28 & & 1 & II & \\
\hline 3831.13 & & 1 & II & & 3797.915 & & 2 & II & & 3770.195 & 2 & 40 & II & \\
\hline 3830.905 & 2 & & I & & 3797.219 & & 2 & II & & 3770.095 & $2000 \mathrm{R}$ & 500 & I & 7 \\
\hline 3830.178 & & 2 & II & & 3797.181 & & 3 & II & & 3769.35 & & 1 & II & \\
\hline 3829.767 & & 2 & II & & 3796.950 & & 10 & II & 4 & 3769.189 & & 1 & II & \\
\hline 3829.736 & & 1 & II & & 3796.495 & 2 & & I & & 3768.966 & & 2 & II & \\
\hline 3828.673 & 20 & 5 & I & & 3796.157 & 7 & 80 & II & 6 & 3767.891 & & 10 & II & \\
\hline 3827.494 & & 4 & II & & 3795.916 & & 4 & II & & 3767.714 & 8 & 2 & I & \\
\hline 3827.030 & & 2 & II & & 3795.878 & & 3 & II & & 3767.175 & & 1 & II & \\
\hline 3826.768 & & 8 & II & & 3795.785 & 3 & 1 & I & & 3766.925 & 2 & 20 & II & 5 \\
\hline 3826.620 & 5 & 1 & I & & 3795.061 & & 4 & II & & 3766.736 & & $8 \mathrm{~h}$ & II & \\
\hline 3826.51 & & 1 & II & & 3794.906 & & 3 & II & & 3766.527 & & 4 & II & \\
\hline 3824.956 & 1 & & I & & 3793.934 & & 4 & II & & 3766.101 & 20 & 200 & II & 4 \\
\hline 3823.241 & 5 & 1 & I & & 3793.104 & & 3 & II & & 3765.080 & 1 & 8 & II & \\
\hline 3822.216 & & 1 & II & & 3792.86 & & 4 & II & & 3764.59 & 1 & & I & \\
\hline 3822.17 & & 1 & II & & 3792.557 & & 1 & II & & 3764.56 & & $3 \mathrm{~h}$ & II & \\
\hline 3821.526 & & 1 & II & & 3792.39 & & 2 & II & & 3764.03 & & 1 & II & \\
\hline 3821.224 & & 1 & II & & 3791.741 & 7000 & 600 & I & 1 & 3763.35 & & 1 & II & \\
\hline 3820.825 & & 8 & II & & 3791.336 & & 2 & II & & 3763.27 & & 1 & II & \\
\hline 3820.288 & & 5 & II & & 3789.457 & & 6 & II & & 3763.045 & 1 & 6 & II & \\
\hline
\end{tabular}


TABLE 1.-Emission spectra of ytterbium-Continued

\begin{tabular}{|c|c|c|c|c|c|c|c|c|c|c|c|c|c|c|}
\hline \multirow{3}{*}{$\begin{array}{c}\text { Wave- } \\
\text { length } \\
\text { in air }\end{array}$} & \multicolumn{2}{|c|}{ Intensity } & \multirow{3}{*}{ Spectrum } & \multirow{3}{*}{$\begin{array}{c}\text { Zeeman } \\
\text { type }\end{array}$} & \multirow{3}{*}{$\begin{array}{c}\text { Wave- } \\
\text { length } \\
\text { in air }\end{array}$} & \multicolumn{2}{|c|}{ Intensity } & \multirow{3}{*}{ Spectrum } & \multirow{3}{*}{$\begin{array}{c}\text { Zeeman } \\
\text { type }\end{array}$} & \multirow{3}{*}{$\begin{array}{c}\text { Wave- } \\
\text { length } \\
\text { in air }\end{array}$} & Inte & nsity & & \\
\hline & Meggers & Thompson & & & & Meggers & Thompson & & & & Meggers & Thompson & spectrom & type \\
\hline & lamp & lamp & & & & lamp & lamp & & & & lamp & lamp & & \\
\hline 3762.98 & & 1 & II & & 3735.606 & & 9 & II & & 3708.218 & & 3 & II & \\
\hline 3762.93 & 1 & & I & & 3735.352 & & 10 & II & & 3707.707 & & 2 & II & \\
\hline 3762.550 & 40 & 400 & II & 4 & 3734.96 & & 1 & II & & 3707.486 & 1 & 7 & II & 5 \\
\hline 3762.12 & & 1 & II & & 3734.694 & $3000 R$ & 300 & I & 1 & 3707.234 & & 1 & II & \\
\hline 3761.31 & & 1 & II & & 3734.435 & 1000 & 100 & I & 1 & 3707.020 & & 4 & II & \\
\hline 3760.999 & 10 & 200 & II & 6 & 3734.150 & & 8 & II & & 3706.833 & & 4 & II & \\
\hline 3760.481 & 200 & 6 & I & & 3734.12 & & 2 & II & & 3706.32 & & 1 & II & \\
\hline 3760.23 & & $2 \mathrm{~d}$ & II & & 3733.989 & & 10 & II & & 3706.023 & 10 & 5 & I & \\
\hline 3757.355 & & 1 & II & & 3733.762 & & 5 & II & & 3705.745 & 1 & & I & \\
\hline 3756.888 & & 2 & II & & 3733.19 & & 1 & II & & 3705.64 & 2 & 30 & II & 7 \\
\hline 3756.445 & & 6 & II & & 3732.458 & & 5 & II & & 3704.750 & & 20 & II & \\
\hline 3755.762 & & 2 & II & & 3732.33 & 1 & 10 & II & & 3704.300 & & 1 & II & \\
\hline 3755.32 & & 1 & II & & 3731.166 & & 7 & II & & 3703.92 & & 7 & II & \\
\hline 3754.83 & & 2 & II & & 3731.044 & & 8 & II & & 3703.423 & 10 & 200 & II & 7 \\
\hline 3754.74 & & 1 & II & & 3730.409 & 20 & 200 & II & 4 & 3702.94 & & 6 & II & \\
\hline 3754.606 & 2 & 6 & II & & 3729.81 & 10 & 100 & II & 4 & 3702.42 & 1 & 5 & II & \\
\hline 3753.897 & 8 & 100 & II & 5 & 3728.24 & & 2 & II & & 3702.07 & & 8 & II & \\
\hline 3753.65 & & 1 & II & & 3727.073 & & 1 & II & & 3700.580 & 1000 & 40 & I & 2 \\
\hline 3753.49 & & 2 & II & & 3726.169 & 1 & 5 & II & & 3699.800 & 5 & 100 & II & 7 \\
\hline 3753.053 & 4 & 60 & II & & 3725.84 & 5 & 70 & II & & 3699.514 & 3000 & 100 & I & \\
\hline 3752.730 & & 4 & II & & 3724.91 & & 3 & II & & 3698.81 & 2 & & I & \\
\hline 3752.56 & & 1 & II & & 3724.36 & 200 & & I & & 3698.596 & 40 & 400 & II & 6 \\
\hline 3751.847 & 10 & 40 & II & 6 & 3724.213 & 150 & 800 & II & 5 & 3698.446 & 2 & 40 & II & \\
\hline 3751.62 & 1 & & I & & 3723.06 & & 2 & II & & 3698.070 & & 2 & II & \\
\hline 3751.206 & 1 & 2 & II & & 3722.626 & & 7 & II & & 3698.012 & & 6 & II & \\
\hline 3751.13 & 1 & & I & & 3722.296 & 40 & 600 & II & 7 & 3697.688 & & 2 & II & \\
\hline 3751.030 & & 1 & II & & 3720.98 & 6 & 100 & II & 5 & 3694.190 & $100000 \mathrm{~A}$ & 80000 & II & 6 \\
\hline 3750.045 & & 8 & II & & 3720.846 & & 3 & II & & 3692.072 & & 4 & II & \\
\hline 3749.688 & 40 & 400 & II & 5 & 3720.584 & & 2 & II & & 3691.67 & 2 & 6 & II & \\
\hline 3749.388 & 1 & 6 & II & & 3720.192 & & 3 & II & & 3691.472 & 10 & 200 & II & 6 \\
\hline 3748.55 & 1 & & I & & 3719.924 & & 3 & II & & 3691.34 & & 1 & II & \\
\hline 3748.345 & 3 & 10 & II & & 3719.148 & & 2 & II & & 3691.230 & 1 & 10 & II & \\
\hline 3748.204 & & 2 & II & & 3718.887 & & 1 & II & & 3690.560 & 30 & 300 & II & 7 \\
\hline 3748.102 & & 1 & II & & 3718.749 & & 2 & II & & 3689.99 & & 8 & II & \\
\hline 3747.817 & 2 & 20 & II & & 3718.482 & 1 & 10 & II & & 3688.937 & & 4 & II & \\
\hline 3747.229 & & 10 & II & & 3718.139 & & 4 & II & & 3688.81 & 10 & & I & \\
\hline 3746.67 & & 1 & II & & 3718.052 & 3 & 15 & II & & 3688.490 & & 1 & II & \\
\hline 3746.33 & 3 & & I & & 3717.885 & & 2 & II & & 3688.438 & 10 & 1 & I & \\
\hline 3744.943 & 40 & 5 & I & & 3717.412 & & 3 & II & & 3687.598 & 20 & 150 & II & 6 \\
\hline 3744.762 & & 1 & II & & 3717.28 & 3 & & I & & 3687.142 & 7 & 80 & II & 6 \\
\hline 3744.166 & & 4 & II & & 3717.23 & 1 & & I & & 3685.973 & & 15 & II & \\
\hline 3744.09 & 2 & & I & & 3717.15 & & 1 & II & & 3684.997 & 500 & 40 & I & 3 \\
\hline 3743.97 & & 1 & II & & 3716.596 & & 2 & II & & 3684.068 & & 1 & II & \\
\hline 3743.390 & & 3 & II & & 3716.139 & 10 & 40 & II & & 3683.352 & 2 & 30 & II & 7 \\
\hline 3743.26 & & 6 & II & & 3715.725 & & 4 & II & & 3683.277 & 1 & 15 & II & \\
\hline 3743.138 & & $15 \mathrm{~h}$ & II & & 3715.440 & & 10 & II & & 3682.777 & & 2 & II & \\
\hline 3742.627 & & 1 & II & & 3715.409 & 50 & 4 & I & & 3682.20 & & 1 & II & \\
\hline 3742.358 & & 1 & II & & 3714.186 & 50 & 150 & II & 6 & 3681.29 & & 1 & II & \\
\hline 3742.036 & 4 & 20 & II & & 3713.93 & & 1 & II & & 3681.195 & & 15 & II & \\
\hline 3741.642 & 3 & 15 & II & & 3713.70 & & 2 & II & & 3680.770 & & 7 & II & \\
\hline 3741.59 & 8 & 80 & II & 4 & 3713.298 & & 2 & II & & 3680.667 & & 4 & II & \\
\hline 3741.296 & $\begin{array}{r}{ }^{\circ} \\
50\end{array}$ & $\begin{array}{r}00 \\
6\end{array}$ & I & & 3712.666 & 10 & & I & & 3679.932 & 200 & 20 & I & \\
\hline 3740.772 & & 10 & II & & 3712.438 & 1 & 10 & II & & 3679.521 & & 2 & II & \\
\hline 3740.59 & & 2 & II & & 3712.416 & & 4 & II & & 3678.26 & & 1 & II & \\
\hline 3739.59 & & 1 & II & & 3712.06 & & 1 & II & & 3678.15 & & 1 & II & \\
\hline 3739.56 & & 1 & II & & 3711.95 & 1 & & I & & 3677.906 & & 6 & II & \\
\hline 3739.411 & 30 & 3 & I & & 3711.907 & 1 & 400 & III & 7 & 3677.853 & 1 & 15 & II & \\
\hline 3738.009 & & 10 & II & & 3711.245 & & 6 & II & & 3677.81 & 2 & & I & \\
\hline 3737.80 & 2 & & I & & 3710.825 & & 3 & II & & 3677.25 & 2 & & I & \\
\hline 3737.537 & 1 & 40 & II & & 3710.340 & 100 & 1000 & II & 6 & 3676.89 & & 1 & II & \\
\hline 3737.123 & & 6 & II & & 3710.090 & & 2 & II & & 3676.14 & & 2 & II & \\
\hline 3736.901 & 7 & 10 & II & 4 & 3708.97 & & 10 & II & & 3675.782 & & 20 & III & \\
\hline 3736.237 & 400 & 100 & I & 3 & 3708.663 & 150 & 600 & II & 4 & 3675.720 & 20 & & I & \\
\hline
\end{tabular}


TABLE 1.-Emission spectra of ytterbium-Continued

\begin{tabular}{|c|c|c|c|c|c|c|c|c|c|c|c|c|c|c|}
\hline \multirow{3}{*}{$\begin{array}{c}\begin{array}{c}\text { Wave- } \\
\text { length } \\
\text { in air }\end{array} \\
\AA \\
\end{array}$} & \multicolumn{2}{|c|}{ Intensity } & \multirow{3}{*}{ Spectrum } & \multirow{3}{*}{$\begin{array}{c}\text { Zeeman } \\
\text { type }\end{array}$} & \multirow{3}{*}{$\begin{array}{c}\text { Wave- } \\
\text { length } \\
\text { in air }\end{array}$} & \multicolumn{2}{|c|}{ Intensity } & \multirow{3}{*}{ Spectrum } & \multirow{3}{*}{$\begin{array}{c}\text { Zeeman } \\
\text { type }\end{array}$} & \multirow{3}{*}{$\begin{array}{c}\text { Wave- } \\
\text { length } \\
\text { in air }\end{array}$} & Inte & nsity & & \\
\hline & Meggers & Thompson & & & & Meggers & Thompson & & & & Meggers & Thompson & vpecinom & type \\
\hline & lamp & lamp & & & & lamp & lamp & & & & lamp & lamp & & \\
\hline 3675.085 & 300 & 2000 & II & 7 & 3647.25 & 1 & & I & & 3614.593 & & 6 & II & \\
\hline 3674.065 & 2 & 20 & II & & 3646.277 & 1 & 10 & II & & 3614.048 & 5 & 100 & II & 4 \\
\hline 3673.900 & & 8 & II & & 3646.24 & & 7 & II & & 3613.894 & & 200 & III & \\
\hline 3673.423 & & 8 & II & & 3645.72 & & 7 & II & & 3613.38 & & 3 & II & \\
\hline 3673.286 & & 1 & II & & 3644.236 & 40 & 400 & II & 5 & $3613: 340$ & & 3 & II & \\
\hline 3670.69 & 50 & 600 & II & 5 & 3643.28 & 1 & 10 & II & & 3613.295 & & 2 & II & \\
\hline 3669.69 & $2000 \mathrm{r}$ & 6000 & II & 6 & 3642.63 & & 3 & II & & 3612.950 & & 4 & II & \\
\hline 3668.992 & 3 & 50 & II & & 3642.37 & & $7 \mathrm{~h}$ & II & & 3611.994 & 20 & 1 & I & \\
\hline 3668.16 & & 1 & II & & 3642.24 & & 4 & II & & 3611.300 & 60 & 800 & II & 4 \\
\hline 3667.951 & & 8 & II & & 3640.79 & & 1 & II & & 3610.233 & 100 & 700 & II & 6 \\
\hline 3666.67 & 1 & 30 & II & & 3639.97 & & 1 & II & & 3609.24 & & 1 & II & \\
\hline 3666.62 & 200 & 4 & I & 7 & 3639.746 & 1 & 15 & II & & 3608.936 & & 3 & II & \\
\hline 3666.522 & & 5 & II & & 3639.445 & 20 & 8 & I & & 3608.488 & 10 & 150 & II & 5 \\
\hline 3666.09 & & 10 & II & & 3639.030 & & 2 & II & & 3608.356 & 3 & & I & \\
\hline 3664.895 & 3 & 40 & II & & 3637.757 & $2000 \mathrm{R}$ & 3000 & II & 6 & 3607.703 & 700 & 70 & I & \\
\hline 3664.744 & 40 & 200 & II & 5 & 3636.899 & & 2 & II & & 3607.186 & & 2 & II & \\
\hline 3664.560 & 8 & 90 & II & 7 & 3636.23 & & 2 & II & & 3606.478 & 300 & 2000 & II & 5 \\
\hline 3663.74 & & 30 & III & & 3635.330 & & 1 & II & & 3605.347 & 2 & 20 & II & 6 \\
\hline 3663.11 & & 6 & II & & 3634.86 & & 2 & II & & 3605.208 & & 1 & II & \\
\hline 3662.545 & 1 & 20 & II & & 3634.74 & & 1 & II & & 3603.848 & 20 & 300 & II & 5 \\
\hline 3662.297 & & 2 & II & & 3634.525 & 30 & 20 & $\mathrm{I}, \mathrm{II}$ & & 3602.237 & & 1 & II & \\
\hline 3662.061 & & 7 & II & & 3634.40 & & 10 & II & & 3601.917 & 2 & 20 & II & 6 \\
\hline 3662.033 & & 5 & II & & 3634.27 & & 2 & II & & 3601.209 & 2 & 15 & II & \\
\hline 3661.656 & & 2 & II & & 3633.858 & & 10 & II & & 3600.966 & 10 & 100 & II & \\
\hline 3661.135 & & 2 & II & & 3633.70 & 1 & 20 & II & 7 & 3600.763 & 20 & 300 & II & \\
\hline 3661.08 & & 6 & II & & 3632.595 & 20 & 150 & II & 5 & 3600.663 & 2 & 30 & II & \\
\hline 3660.99 & & 1 & II & & 3632.415 & 20 & 100 & II & 5 & 3600.383 & 10 & 150 & II & 6 \\
\hline 3660.73 & 1 & 8 & II & & 3631.86 & & 8 & II & & 3598.87 & & $2 \mathrm{~h}$ & II & \\
\hline 3660.64 & & 1 & II & & 3631.073 & 7 & 80 & II & 4 & 3598.662 & 9 & 1 & I & \\
\hline 3660.58 & 1 & 15 & II & & 3630.975 & 4 & & I & & 3598.043 & 2 & 20 & II & \\
\hline 3660.42 & & 1 & II & & 3630.621 & & 4 & II & & 3597.60 & & 1 & II & \\
\hline 3660.137 & 1 & 10 & II & & 3630.337 & & 2 & II & & 3597.078 & & 10 & II & \\
\hline 3659.835 & & 30 & III & & 3630.301 & & 4 & II & & 3596.488 & 1 & 30 & II & \\
\hline 3659.427 & 4 & & I & & 3629.919 & 30 & 300 & II & 6 & 3596.416 & 3 & & I & \\
\hline 3658.78 & 1 & & I & & 3629.58 & 3 & & I & & 3596.300 & & 4 & II & \\
\hline 3658.605 & 5 & 70 & II & & 3629.233 & 800 & 15 & I & 7 & 3595.844 & & 5 & II & \\
\hline 3657.665 & & 3 & II & & 3628.340 & & 6 & II & & 3595.649 & & 2 & II & \\
\hline 3657.233 & $3 \mathrm{~h}$ & & I & & 3627.89 & 2 & 30 & II & & 3595.162 & & 9 & II & \\
\hline 3657.131 & & 1 & II & & 3627.205 & 2 & 30 & II & 5 & 3594.93 & 1 & & I & \\
\hline 3657.007 & & 2 & II & & 3627.078 & & 1 & II & & 3594.864 & 1 & 10 & II & \\
\hline 3656.81 & & 1 & II & & 3626.80 & 1 & 6 & II & & 3593.882 & & 1 & II & \\
\hline 3656.672 & & 2 & II & & 3625.230 & 2 & 60 & II & & 3593.728 & & 2 & II & \\
\hline 3656.312 & 10 & 2 & I & & 3624.634 & 15 & 200 & II & 6 & 3593.060 & & 1 & II & \\
\hline 3655.729 & $2000 \mathrm{R}$ & 100 & I & 1 & 3624.109 & 10 & 1 & I & & 3592.734 & 1 & 20 & II & \\
\hline 3655.48 & 1 & 20 & II & & 3623.627 & 1 & 10 & II & & 3592.217 & 1 & 10 & II & \\
\hline 3654.417 & 1 & 5 & II & & 3622.320 & 20 & 80 & II & 6 & 3591.844 & 150 & 15 & I & \\
\hline 3654.28 & 1 & 20 & II & & 3621.873 & & 7 & II & & 3591.24 & & 1 & II & \\
\hline 3653.041 & 2 & 20 & II & & 3621.80 & & 2 & II & & 3591.18 & & 2 & II & \\
\hline 3652.953 & 3 & 40 & II & & 3621.743 & 400 & 10 & I & & 3591.141 & & 4 & II & \\
\hline 3652.538 & 1 & 15 & II & & 3621.513 & 1 & 15 & II & & 3590.657 & 5 & 50 & II & 7 \\
\hline 3651.24 & 1 & 10 & II & & 3621.18 & & 1 & II & & 3589.997 & & 1 & II & \\
\hline 3651.15 & & 1 & II & & 3620.988 & 30 & 400 & II & 7 & 3589.186 & & 7 & II & \\
\hline 3650.80 & 4 & 50 & II & 4 & 3620.65 & 2 & & I & & 3588.832 & 1 & 20 & II & \\
\hline 3649.739 & 10 & 150 & II & 5 & 3620.362 & & 4 & II & & 3588.456 & & 2 & II & \\
\hline 3649.084 & & 6 & II & & 3619.803 & $700 \mathrm{r}$ & 3000 & II & 4 & 3587.736 & & 7 & II & \\
\hline 3648.827 & & 2 & II & & 3618.887 & & 1 & II & & 3587.569 & & 1 & II & \\
\hline 3648.676 & & 1 & II & & 3618.040 & 50 & 200 & II & 6 & 3586.824 & 30 & 90 & II & 6 \\
\hline 3648.440 & 10 & 100 & II & 6 & 3617.045 & 1 & 10 & II & & 3586.536 & & 6 & II & \\
\hline 3648.38 & 4 & 50 & II & & 3616.395 & & 10 & II & & 3585.878 & & 8 & II & \\
\hline 3648.29 & & 1 & II & & 3616.031 & & 3 & II & & 3585.794 & 1 & 20 & II & \\
\hline 3648.242 & & 2 & II & & 3615.957 & & 2 & II & & 3585.466 & $2000 \mathrm{R}$ & 4000 & II & 5 \\
\hline 3648.150 & 150 & 15 & I & & 3614.994 & 50 & 7 & I & 7 & 3583.96 & & $4 \mathrm{~h}$ & II & \\
\hline 3647.730 & 2 & 40 & II & 7 & 3614.63 & & 1 & II & & 3583.827 & 7 & 70 & II & \\
\hline
\end{tabular}


TABLE 1.-Emission spectra of ytterbium-Continued

\begin{tabular}{|c|c|c|c|c|c|c|c|c|c|c|c|c|c|c|}
\hline \multirow{3}{*}{$\begin{array}{l}\text { Wave- } \\
\text { length } \\
\text { in air }\end{array}$} & \multicolumn{2}{|c|}{ Intensity } & \multirow{3}{*}{ Spectrum } & \multirow{3}{*}{$\begin{array}{l}\text { Zeeman } \\
\text { type }\end{array}$} & \multirow{3}{*}{$\begin{array}{l}\text { Wave- } \\
\text { length } \\
\text { in air } \\
\\
\text { A }\end{array}$} & \multicolumn{2}{|c|}{ Intensity } & \multirow{3}{*}{ Spectrum } & \multirow{3}{*}{$\begin{array}{c}\text { Zeeman } \\
\text { type }\end{array}$} & \multirow{3}{*}{$\begin{array}{c}\text { Wave- } \\
\text { length } \\
\text { in air }\end{array}$} & Inte & nsity & & \\
\hline & Meggers & Thompson & & & & Meggers & Thompson & & & & Meggers & Thompson & Jpocirom & type \\
\hline & lamp & lamp & & & & lamp & lamp & & & & lamp & lamp & & \\
\hline 3583.532 & & 5 & II & & 3556.435 & & 3 & II & & 3525.171 & & 2 & II & \\
\hline 3583.26 & & 7 & II & & 3556.263 & 200 & 15 & I & & 3525.05 & & 1 & II & \\
\hline 3583.180 & & 1 & II & & 3556.124 & & 1 & II & & 3524.82 & & 1 & II & \\
\hline 3582.841 & 3 & 40 & II & & 3556.042 & & 1 & II & & 3524.044 & & 1 & II & \\
\hline 3582.20 & & 3 & II & & 3556.013 & 10 & & I & & 3523.734 & & $3 \mathrm{~h}$ & II & \\
\hline 3580.755 & & 10 & II & & 3555.204 & 5 & 30 & II & 4 & 3523.148 & 3 & 2 & I,II & \\
\hline 3580.640 & 1 & 15 & II & & 3555.055 & & 3 & II & & 3522.826 & & 3 & II & \\
\hline 3580.474 & & 1 & II & & 3554.347 & & 5 & II & & 3522.747 & 1 & 10 & II & \\
\hline 3580.404 & 100 & 10 & I & & 3553.82 & & 1 & II & & 3522.021 & & 1 & II & \\
\hline 3580.125 & 100 & 20 & I & & 3553.340 & & 7 & II & & 3521.773 & 5 & & I & \\
\hline 3579.511 & 3 & 40 & II & 7 & 3553.192 & & 3 & II & & 3521.727 & & 3 & II & \\
\hline 3578.95 & & 1 & II & & 3553.153 & & 3 & II & & 3521.664 & & 2 & II & \\
\hline 3578.710 & 10 & 2 & I & & 3552.324 & 100 & 1000 & II & 5 & 3521.585 & 1 & 7 & II & \\
\hline 3578.561 & 600 & 60 & I & 1 & 3551.405 & 1 & & I & & 3521.224 & & 7 & II & \\
\hline 3577.985 & & 1 & II & & 3550.87 & & 300 & III & & 3521.007 & 5 & 20 & II & \\
\hline 3577.690 & 1 & 10 & II & & 3549.822 & 200 & 2000 & II & 6 & 3520.917 & & 10 & II & \\
\hline 3577.504 & & 3 & II & & 3548.470 & & 2 & II & & 3520.293 & $600 \mathrm{r}$ & 3000 & II & 6 \\
\hline 3577.008 & 30 & 300 & II & 7 & 3548.18 & 50 & 7 & I & & 3519.82 & 2 & & I & \\
\hline 3576.288 & & 8 & II & & 3548.096 & & 7 & II & & 3519.690 & & 2 & II & \\
\hline 3576.188 & & 1 & II & & 3547.756 & 5 & 1 & I & & 3519.417 & 5 & 50 & II & 7 \\
\hline 3575.905 & 1 & 20 & II & & 3546.085 & & 1 & II & & 3519.10 & & 1 & II & \\
\hline 3575.704 & & 5 & II & & 3545.725 & 1 & $40 \mathrm{~h}$ & II & & 3518.89 & & 1 & II & \\
\hline 3575.573 & 3 & 60 & II & 5 & 3544.162 & 10 & 1 & I & & 3518.795 & & 3 & II & \\
\hline 3575.125 & 20 & 2 & I & & 3543.890 & & 1 & II & & 3518.674 & & 1 & II & \\
\hline 3574.58 & & $30 \mathrm{~h}$ & II & & 3543.324 & 1 & 10 & II & & 3518.457 & 300 & 20 & I & 1 \\
\hline 3573.750 & 1 & 10 & II & 7 & 3543.159 & 30 & 200 & II & 4 & 3518.353 & 1 & 10 & II & \\
\hline 3573.67 & & 1 & II & & 3542.385 & 7 & 100 & II & 6 & 3518.156 & 20 & 300 & II & 7 \\
\hline 3573.458 & 3 & 3 & I,II & & 3542.272 & 1 & 6 & II & & 3517.001 & 500 & 60 & $\mathbf{i}$ & 3 \\
\hline 3573.41 & & 4 & II & & 3541.924 & 8 & 1 & I & & 3516.60 & & 1 & II & \\
\hline 3573.247 & 20 & 2 & I & & 3541.375 & 7 & 80 & II & 5 & 3516.180 & & 2 & II & \\
\hline 3572.498 & 200 & 800 & II & 4 & 3540.926 & & 3 & II & & 3515.865 & 30 & 300 & II & 4 \\
\hline 3572.448 & 50 & & I & & 3540.61 & & 1 & II & & 3515.610 & 10 & 50 & II & 6 \\
\hline 3572.212 & 2 & 15 & II & & 3540.21 & & 2 & II & & 3515.238 & 20 & 2 & I & \\
\hline 3570.987 & 1 & 8 & II & & 3540.09 & & 1 & II & & 3515.138 & 1 & 15 & II & \\
\hline 3570.566 & 200 & 1000 & II & 5 & 3540.02 & & 1 & II & & 3513.640 & & 4 & II & \\
\hline 3569.925 & 2 & 20 & II & & 3539.932 & & 3 & II & & 3513.573 & 400 & 30 & I & 3 \\
\hline 3569.500 & 2 & & I & & 3539.362 & 20 & 200 & II & 4 & 3513.412 & & 2 & II & \\
\hline 3569.308 & & 2 & II & & 3539.33 & 200 & & I & & 3512.708 & 2 & 20 & II & \\
\hline 3569.094 & 1 & 10 & II & & 3538.369 & & 2 & II & & 3512.441 & & 6 & II & \\
\hline 3568.430 & 5 & 50 & II & 4 & 3537.964 & & 1 & II & & 3511.283 & & 8 & II & \\
\hline 3567.130 & 50 & 500 & II & 6 & 3537.127 & & 6 & II & & 3510.942 & & 3 & II & \\
\hline 3566.905 & 2 & 1 & I & & 3536.815 & $3 \mathrm{~h}$ & & I & & 3510.764 & 600 & 50 & I & 1 \\
\hline 3566.519 & & 2 & II & & 3536.068 & 1 & 8 & II & & 3510.500 & 1 & 15 & II & \\
\hline 3565.891 & & 4 & II & & 3534.752 & 4 & 20 & II & & 3509.86 & 1 & 10 & II & \\
\hline 3565.823 & & 1 & II & & 3534.13 & & 2 & II & & 3509.776 & & 3 & II & \\
\hline 3565.477 & & 6 & II & & 3534.05 & 50 & 100 & II & 5 & 3508.95 & & 1 & II & \\
\hline 3565.404 & & 3 & II & & 3533.80 & & 3 & II & & 3507.830 & 200 & 2000 & II & 4 \\
\hline 3564.955 & & 1 & II & & 3533.107 & 20 & 60 & II & 6 & 3506.125 & 8 & 200 & II & 5 \\
\hline 3563.941 & 200 & 800 & II & 5 & 3531.725 & 20 & 1 & I & & 3505.282 & 50 & 150 & II & 4 \\
\hline 3563.712 & 2 & 20 & II & & 3531.24 & 5 & 100 & II & 4 & 3504.936 & 3 & 15 & II & \\
\hline 3562.706 & 7 & 80 & II & 6 & 3530.58 & & 1 & II & & 3504.737 & 3 & & I & \\
\hline 3561.738 & 1 & 10 & II & & 3530.16 & & 3 & II & & 3504.542 & 1 & 15 & II & \\
\hline 3561.444 & 2 & 6 & II & & 3530.05 & & 1 & II & & 3503.988 & 20 & 7 & I & \\
\hline 3561.309 & & 2 & II & & 3529.715 & 2 & 20 & II & & 3503.690 & 4 & 40 & II & 4 \\
\hline 3560.704 & $500 \mathrm{r}$ & 2000 & II & 6 & 3529.458 & & 2 & II & & 3503.277 & & 2 & II & \\
\hline 3560.327 & $2000 \mathrm{R}$ & 3000 & II & 7 & 3529.085 & 8 & 100 & II & 6 & 3503.190 & & 3 & II & \\
\hline 3559.570 & 10 & & I & & 3528.771 & & 2 & II & & 3503.011 & & 1 & II & \\
\hline 3559.139 & & 4 & II & & 3528.075 & 2 & & I & & 3502.572 & & $4 \mathrm{~h}$ & II & \\
\hline 3559.032 & 1000 & 50 & I & 7 & 3527.501 & 1 & 15 & II & 7 & 3502.180 & 10 & 100 & II & 4 \\
\hline 3557.825 & & 2 & II & & 3527.332 & 2 & 20 & II & 7 & 3500.863 & & 5 & II & \\
\hline 3557.466 & & 1 & II & & 3526.477 & & 1 & II & & 3500.813 & 10 & 5 & I & \\
\hline 3557.100 & 2 & 20 & II & 7 & 3525.722 & 5 & 50 & II & 6 & 3500.131 & 10 & 30 & II & \\
\hline 3556.940 & & 4 & II & & 3525.456 & & 3 & II & & 3499.886 & & 3 & II & \\
\hline
\end{tabular}


TABLE 1.-Emission spectra of ytterbium-Continued

\begin{tabular}{|c|c|c|c|c|c|c|c|c|c|c|c|c|c|c|}
\hline \multirow{3}{*}{$\begin{array}{c}\text { Wave- } \\
\text { length } \\
\text { in air }\end{array}$} & \multicolumn{2}{|c|}{ Intensity } & \multirow{3}{*}{ Spectrum } & \multirow{3}{*}{$\begin{array}{l}\text { Zeeman } \\
\text { type }\end{array}$} & \multirow{3}{*}{$\begin{array}{c}\text { Wave- } \\
\text { length } \\
\text { in air }\end{array}$} & \multicolumn{2}{|c|}{ Intensity } & \multirow{3}{*}{ Spectrum } & \multirow{3}{*}{$\begin{array}{c}\text { Zeeman } \\
\text { type }\end{array}$} & \multirow{3}{*}{$\begin{array}{l}\text { Wave- } \\
\text { length } \\
\text { in air }\end{array}$} & Inte & ensity & & \\
\hline & Meggers & Thompson & & & & Meggers & Thompson & & & & Meggers & & spectrom & type \\
\hline & lamp & lamp & & & & lamp & lamp & & & & lamp & lamp & & \\
\hline 3499.85 & & 1 & II & & 3468.00 & & $3 \mathrm{~h}$ & II & & 3442.368 & 3 & 40 & II & 6 \\
\hline 3499.791 & & 2 & II & & 3467.26 & 10 & & I & & 3442.058 & 1 & 10 & II & \\
\hline 3499.636 & 1 & 9 & II & & 3467.066 & 10 & 200 & II & 7 & 3441.354 & & 7 & II & \\
\hline 3499.419 & 5 & 10 & II & & 3466.346 & & 10 & II & & 3441.30 & & 1 & II & \\
\hline 3498.392 & 2 & 40 & II & & 3465.898 & 3 & 40 & II & 6 & 3441.07 & 3 & & I & \\
\hline 3497.86 & & 2 & II & & 3465.792 & & 3 & II & & 3440.894 & & $8 \mathrm{~h}$ & II & \\
\hline 3496.858 & 5 & 20 & II & & 3464.905 & & 1 & II & & 3440.016 & & 5 & II & \\
\hline 3495.90 & & $50 \mathrm{~h}$ & II & & 3464.37 & $30000 \mathrm{~A}$ & 2000 & I & 7 & 3439.648 & 1 & 20 & II & \\
\hline 3495.780 & & 3 & II & & 3464.190 & 100 & 20 & I & & 3438.848 & $400 \mathrm{r}$ & 2000 & II & 6 \\
\hline 3495.623 & 10 & 1 & I & & 3463.511 & & 150 & III & & 3438.709 & 200 & 1500 & II & 6 \\
\hline 3493.154 & 1 & 15 & II & & 3463.325 & 2 & 30 & II & & 3438.516 & & 10 & II & \\
\hline 3492.807 & 5 & 30 & II & & 3463.179 & 2 & 20 & II & & 3437.754 & & 3 & II & \\
\hline 3491.624 & 6 & 100 & II & & 3462.79 & & $1 \mathrm{~h}$ & II & & 3437.222 & & 2 & II & \\
\hline 3491.133 & 2 & & I & & 3462.338 & 20 & 15 & I & & 3437.17 & 5 & 1 & I & \\
\hline 3491.063 & & 3 & II & & 3461.719 & 30 & 300 & II & 4 & 3437.114 & & 2 & II & \\
\hline 3490.880 & & $2 \mathrm{~h}$ & II & & 3461.327 & & 5 & II & & 3436.452 & 100 & 700 & II & 5 \\
\hline 3490.58 & & 1 & II & & 3461.004 & 100 & 9 & I & & 3435.74 & & $4 \mathrm{~h}$ & II & \\
\hline 3490.213 & 1 & 20 & II & & 3460.269 & $3000 \mathrm{R}$ & 60 & I & 7 & 3435.516 & & 2 & II & \\
\hline 3489.746 & 60 & 15 & I & & 3460.137 & & 4 & II & & 3435.429 & & 3 & II & \\
\hline 3489.399 & 8 & 1 & 1 & & 3460.092 & & 10 & II & & 3434.610 & 200 & 600 & II & 5 \\
\hline 3488.855 & 200 & 1 & I & & 3459.902 & & 2 & II & & 3434.244 & 1 & 10 & II & \\
\hline 3488.786 & 200 & 700 & II & 5 & 3459.828 & & 4 & II & & 3433.650 & & 1 & II & \\
\hline 3488.43 & & $10 \mathrm{~h}$ & II & & 3459.663 & 100 & 10 & I & 7 & 3433.167 & 100 & 10 & I & \\
\hline 3488.34 & 6 & 50 & II & & 3459.183 & 10 & 40 & II & 4 & 3433.160 & & 10 & II & \\
\hline 3487.971 & 20 & 5 & I & & 3459.095 & 60 & 3 & I & & 3432.942 & & 80 & III & \\
\hline 3487.603 & 2 & & I & & 3458.792 & & 2 & II & & 3432.732 & 1 & 3 & II & \\
\hline 3487.402 & & 3 & II & & 3458.391 & 1000 & 20 & I & 7 & 3432.261 & 1 & 30 & II & \\
\hline 3487.233 & 6 & 100 & II & & 3458.286 & 200 & 1500 & II & 5 & 3432.143 & 150 & 15 & I & \\
\hline 3487.041 & & 2 & II & & 3457.333 & & 2 & II & & 3431.780 & & $4 \mathrm{~h}$ & II & \\
\hline 3486.137 & 1 & 8 & II & & 3457.10 & & 2 & II & & 3431.63 & & 1 & II & \\
\hline 3485.757 & 200 & 1500 & II & 7 & 3457.04 & & 1 & II & & 3431.512 & & 2 & II & \\
\hline 3484.656 & & 1 & II & & 3456.953 & & 1 & II & & 3431.377 & 5 & 9 & II & \\
\hline 3484.38 & & 2 & II & & 3456.774 & & 6 & II & & 3431.140 & 700 & 20 & I & 7 \\
\hline 3484.176 & 7 & & I & & 3456.525 & 10 & & I & & 3431.107 & $3000 \mathrm{R}$ & 80 & I & 7 \\
\hline 3484.143 & 1 & 15 & II & & 3456.231 & 5 & 20 & II & & 3430.872 & & 3 & II & \\
\hline 3484.09 & & 2 & II & & 3456.181 & & 40 & III & & 3430.65 & & 1 & II & \\
\hline 3484.06 & & 2 & II & & 3455.863 & & 5 & II & & 3430.632 & & 2 & II & \\
\hline 3483.782 & 1 & 10 & II & & 3454.92 & & 3 & II & & 3429.96 & & 1 & II & \\
\hline 3482.942 & 1 & 10 & II & & 3454.75 & 10 & & I & & 3429.80 & & 1 & II & \\
\hline 3482.564 & 5 & $30 \mathrm{~h}$ & II & & 3454.215 & 50 & 300 & II & & 3428.782 & 700 & 30 & I & 7 \\
\hline 3482.390 & & 1 & II & & 3454.080 & $2000 \mathrm{r}$ & 10000 & II & 4 & 3428.463 & 200 & 2000 & II & 6 \\
\hline 3481.706 & & 2 & II & & 3453.524 & & 5 & II & & 3427.808 & 1 & 20 & II & \\
\hline 3481.68 & 30 & & I & & 3453.207 & & 2 & II & & 3427.508 & & 3 & II & \\
\hline 3481.302 & 1 & 20 & II & & 3452.398 & 300 & 30 & I & 3 & 3426.878 & 150 & 30 & I & \\
\hline 3479.912 & & 2 & II & & 3452.264 & & 7 & II & & 3426.558 & & 2 & II & \\
\hline 3478.835 & $3000 \mathrm{r}$ & 10000 & II & 5 & 3451.82 & 2 & & I & & 3426.43 & & 1 & II & \\
\hline 3477.022 & 20 & 30 & II & & 3451.33 & 1 & 20 & II & & 3426.044 & $3000 \mathrm{R}$ & 100 & I & 7 \\
\hline 3476.303 & $10000 \mathrm{~A}$ & 8000 & II & 4 & 3451.305 & & 3 & II & & 3425.71 & & 1 & II & \\
\hline 3474.82 & 60 & 400 & II & 4 & 3450.929 & & 2 & II & & 3424.750 & & 5 & II & \\
\hline 3474.512 & & 3 & II & & 3450.269 & & 5 & II & & 3424.610 & 1 & & I & \\
\hline 3473.996 & & 5 & II & & 3449.362 & & 1 & II & & 3424.554 & 3 & 15 & II & \\
\hline 3473.75 & & 3 & II & & 3449.15 & & $7 \mathrm{~h}$ & II & & 3424.295 & 1 & & I & \\
\hline 3473.01 & & 2 & II & & 3448.906 & & 1 & II & & 3424.182 & & 1 & II & \\
\hline 3472.58 & & 1 & II & & 3447.993 & 4 & 40 & II & 7 & 3422.454 & & 6 & II & \\
\hline 3472.324 & 5 & 20 & II & & 3447.668 & 1 & 20 & II & 7 & 3421.409 & & 1 & II & \\
\hline 3471.891 & & 2 & II & & 3446.887 & 100 & 600 & II & 6 & 3420.345 & 150 & 20 & I & \\
\hline 3471.46 & & 1 & II & & 3445.950 & 7 & 100 & II & 5 & 3419.807 & 3 & & I & \\
\hline 3470.79 & 8 & 80 & II & 5 & 3445.107 & & 3 & II & & 3419.742 & 2 & 20 & II & 7 \\
\hline 3470.106 & 10 & 3 & I & & 3444.544 & & 2 & II & & 3419.606 & 20 & 100 & II & 4 \\
\hline 3469.975 & & 20 & III & & 3443.895 & & $2 \mathrm{~h}$ & II & & 3418.571 & & 1 & II & \\
\hline 3469.925 & 5 & 70 & II & 4 & 3443.587 & 30 & 20 & I,II & 7 & 3418.390 & 400 & 50 & I & 7 \\
\hline 3469.253 & 3 & 5 & II & & 3443.391 & & $6 \mathrm{~h}$ & II & & 3417.565 & 3 & 30 & II & 5 \\
\hline 3468.395 & 1 & 20 & II & & 3442.586 & 10 & 5 & II & & 3417.345 & 1 & 10 & II & \\
\hline
\end{tabular}


TABLE 1.-Emission spectra of ytterbium-Continued

\begin{tabular}{|c|c|c|c|c|c|c|c|c|c|c|c|c|c|c|}
\hline \multirow{3}{*}{$\begin{array}{c}\text { Wave- } \\
\text { length } \\
\text { in air }\end{array}$} & \multicolumn{2}{|c|}{ Intensity } & \multirow{3}{*}{ Spectrum } & \multirow{3}{*}{$\begin{array}{c}\text { Zeeman } \\
\text { type }\end{array}$} & \multirow{3}{*}{$\begin{array}{c}\text { Wave- } \\
\text { length } \\
\text { in air }\end{array}$} & \multicolumn{2}{|c|}{ Intensity } & \multirow{3}{*}{ Spectrum } & \multirow{3}{*}{$\begin{array}{c}\text { Zeeman } \\
\text { type }\end{array}$} & \multirow{3}{*}{$\begin{array}{c}\text { Wave- } \\
\text { length } \\
\text { in air }\end{array}$} & Inte & nsity & & \\
\hline & Meggers & Thompson & & & & Meggers & Thompson & & & & Meggers & Thompson & Jpectrom & type \\
\hline & lamp & lamp & & & & lamp & lamp & & & & lamp & lamp & & \\
\hline 3417.044 & 250 & 20 & I & & 3389.439 & & 4 & II & & 3363.645 & 10 & 20 & II & \\
\hline 3416.894 & 40 & 200 & II & 4 & 3388.523 & & 7 & II & & 3362.770 & 1 & & I & \\
\hline 3416.314 & & 1 & II & & 3387.760 & & 3 & II & & 3362.705 & 4 & 6 & II & \\
\hline 3415.974 & 10 & 100 & II & 5 & 3387.505 & $200 \mathrm{r}$ & 50 & I & 1 & 3362.438 & 10 & 70 & II & \\
\hline 3415.853 & & 2 & II & & 3387.370 & 20 & 2 & I & & 3362.145 & 1 & & I & \\
\hline 3415.610 & & 6 & II & & 3387.293 & 2 & 20 & II & & 3361.927 & 4 & & I & \\
\hline 3415.062 & 10 & 80 & II & 7 & 3387.120 & & 2 & II & & 3361.573 & 15 & 100 & II & 4 \\
\hline 3414.838 & 20 & 200 & II & 6 & 3387.073 & & 3 & II & & 3361.467 & 1 & 8 & II & \\
\hline 3413.357 & 5 & 15 & II & 5 & 3386.560 & & 1 & II & & 3361.329 & 3 & 30 & II & \\
\hline 3412.507 & & 5 & II & & 3385.883 & 20 & 40 & II & & 3359.49 & & 1 & II & \\
\hline 3412.453 & 80 & 40 & 1 & 7 & 3385.559 & 10 & 100 & II & 6 & 3359.193 & & $3 \mathrm{~h}$ & II & \\
\hline 3411.750 & & 6 & II & & 3385.329 & 2 & & I & & 3358.612 & & 2 & II & \\
\hline 3411.240 & 10 & 8 & I & & 3384.682 & 200 & 15 & I & & 3358.254 & & 50 & III & \\
\hline 3410.880 & & 4 & II & & 3384.560 & & 1 & II & & 3357.802 & 5 & 40 & II & \\
\hline 3410.689 & 1 & 6 & II & & 3384.352 & & 4 & II & & 3356.966 & 20 & 200 & II & 5 \\
\hline 3410.566 & 50 & 60 & II & 7 & 3384.007 & & 2000 & III & 7 & 3356.683 & & 1 & II & \\
\hline 3410.487 & 1 & 10 & II & & 3383.862 & 3 & 20 & II & 6 & 3356.305 & 6 & 40 & II & \\
\hline 3409.875 & 6 & 70 & II & & 3382.537 & 20 & 20 & $\mathrm{I}, \mathrm{II}$ & & 3355.870 & 20 & 200 & II & 6 \\
\hline 3409.568 & 1 & 10 & II & & 3381.255 & & 1 & II & & 3355.618 & 1 & & I & \\
\hline 3409.022 & & 3 & II & & 3381.060 & 2 & 15 & II & & 3354.020 & & 3 & II & \\
\hline 3408.95 & 2 & & I & & 3380.820 & 1 & 20 & II & 4 & 3353.966 & 2 & 30 & II & \\
\hline 3408.516 & 10 & 100 & II & 4 & 3380.644 & & 1 & II & & 3353.733 & 20 & 150 & II & 4 \\
\hline 3408.20 & & 3 & II & & 3380.340 & & 1 & II & & 3353.607 & 1 & & I & \\
\hline 3408.058 & & 7 & II & & 3379.986 & 40 & 100 & II & 5 & 3353.563 & 1 & 6 & II & \\
\hline 3407.922 & 2 & & I & & 3379.788 & 100 & 700 & II & 4 & 3353.200 & 3 & 40 & II & 4 \\
\hline 3406.536 & 1 & 6 & II & & 3379.517 & 6 & 70 & II & 5 & 3352.880 & 60 & 4 & I & \\
\hline 3406.200 & 200 & 5 & I & & 3379.386 & 2 & 2 & I,II & & 3352.491 & 10 & 30 & II & \\
\hline 3405.79 & & 1 & II & & 3378.698 & 50 & 3 & I & & 3351.382 & 6 & & I & \\
\hline 3405.249 & 5 & 30 & II & & 3378.404 & 10 & 100 & II & 6 & 3351.265 & 20 & 20 & $\mathrm{I}, \mathrm{II}$ & \\
\hline 3405.093 & & 2 & II & & 3378.286 & 60 & 4 & I & & 3351.087 & 6 & 60 & II & 5 \\
\hline 3405.03 & & 1 & II & & 3377.792 & 3 & 40 & II & & 3350.967 & 5 & 40 & II & 5 \\
\hline 3404.103 & 150 & 800 & II & 5 & 3377.456 & & 3 & II & & 3350.357 & 1 & & I & \\
\hline 3402.640 & & 5 & II & & 3376.780 & & 2 & II & & 3350.209 & 3 & & I & \\
\hline 3402.273 & 200 & 300 & II & 6 & 3376.623 & 3 & 30 & II & & 3349.942 & 20 & 150 & II & 7 \\
\hline 3401.010 & 200 & 1000 & II & 5 & 3375.483 & $2000 \mathrm{r}$ & 5000 & II & 6 & 3349.713 & 1 & & I & \\
\hline 3400.603 & & $4 \mathrm{~d}$ & II & & 3375.079 & 30 & 70 & II & & 3349.074 & 10 & 60 & II & 4 \\
\hline 3400.445 & & 3 & II & & 3374.184 & & 1 & II & & 3347.537 & 150 & 1000 & II & 7 \\
\hline 3399.548 & & 3 & II & & 3374.07 & & 1 & II & & 3347.016 & 2 & & I & \\
\hline 3398.649 & 2 & 10 & II & & 3373.873 & 2 & 20 & II & & 3346.504 & 30 & 30 & $\mathrm{I}, \mathrm{II}$ & \\
\hline 3397.661 & & 100 & III & & 3373.736 & & 1 & II & & 3346.192 & & 7 & II & \\
\hline 3397.446 & 2 & 20 & II & & 3373.033 & 1 & 10 & II & & 3345.958 & 2 & & I & \\
\hline 3397.125 & 2 & 20 & II & & 3372.576 & 500 & 40 & I & & 3345.921 & & 7 & II & \\
\hline 3397.037 & 50 & 2 & I & & 3372.00 & & 1 & II & & 3345.575 & 5 & & I & \\
\hline 3396.328 & 40 & 400 & II & 5 & 3371.325 & 100 & 10 & I & & 3345.187 & & 9 & II & \\
\hline 3395.14 & & $5 \mathrm{~h}$ & II & & 3371.090 & 2 & & I & & 3345.012 & 20 & & I & \\
\hline 3394.519 & 3 & & I & & 3369.886 & 10 & 80 & II & 6 & 3344.715 & & 3 & II & \\
\hline 3394.437 & 60 & 300 & II & 5 & 3369.544 & 70 & 200 & II & 5 & 3344.507 & 1 & & I & \\
\hline 3394.152 & & 1 & II & & 3369.344 & 20 & 60 & II & 5 & 3344.212 & 30 & 3 & I & \\
\hline 3393.972 & 30 & 3 & I & & 3367.738 & & 6 & II & & 3343.78 & & 1 & II & \\
\hline 3393.815 & & 1 & II & & 3367.324 & 1 & & I & & 3343.705 & & 2 & II & \\
\hline 3393.636 & & 2 & II & & 3367.077 & & 4 & II & & 3343.566 & & 2 & II & \\
\hline 3393.252 & & 1 & II & & 3366.964 & & 3 & II & & 3343.071 & 80 & 400 & II & 6 \\
\hline 3392.555 & & 150 & III & & 3366.937 & 3 & & I & & 3342.93 & $20 \mathrm{~h}$ & $100 \mathrm{~h}$ & II & \\
\hline 3392.390 & 7 & 100 & II & 5 & 3366.923 & 10 & & I & & 3342.310 & & 1 & II & \\
\hline 3392.356 & 2 & 20 & II & & 3365.969 & 80 & 800 & II & 6 & 3341.097 & 3 & 40 & II & 5 \\
\hline 3392.276 & & 4 & II & & 3365.658 & & 2 & II & & 3340.791 & & 1 & II & \\
\hline 3391.104 & 100 & 900 & II & 4 & 3365.444 & & 1 & II & & 3339.644 & 2 & & I & \\
\hline 3390.817 & 3 & 40 & II & & 3365.181 & 5 & & I & & 3339.476 & & 3 & II & \\
\hline 3390.63 & & 1 & II & & 3365.059 & & 4 & II & & 3339.305 & & 3 & II & \\
\hline 3390.421 & $1 \mathrm{~h}$ & $30 \mathrm{~h}$ & II & & 3364.471 & & 4 & II & & 3339.274 & & 1 & II & \\
\hline 3390.246 & $1 \mathrm{~h}$ & $40 \mathrm{~h}$ & II & & 3364.302 & & 20 & III & & 3339.075 & $10 \mathrm{~h}$ & & I & \\
\hline 3389.985 & & 2 & II & & 3364.125 & & 2 & II & & 3339.022 & & 1 & II & \\
\hline 3389.904 & $8 \mathrm{~h}$ & & I & & 3363.886 & & 1 & II & & 3338.749 & 1 & 8 & II & \\
\hline
\end{tabular}


TABLE 1.-Emission spectra of ytterbium-Continued

\begin{tabular}{|c|c|c|c|c|c|c|c|c|c|c|c|c|c|c|}
\hline \multirow{3}{*}{$\begin{array}{c}\begin{array}{c}\text { Wave- } \\
\text { length } \\
\text { in air }\end{array} \\
\AA \\
\end{array}$} & \multicolumn{2}{|c|}{ Intensity } & \multirow{3}{*}{ Spectrum } & \multirow{3}{*}{$\begin{array}{c}\text { Zeeman } \\
\text { type }\end{array}$} & \multirow{3}{*}{$\begin{array}{c}\begin{array}{c}\text { Wave- } \\
\text { length } \\
\text { in air }\end{array} \\
\AA \\
\end{array}$} & \multicolumn{2}{|c|}{ Intensity } & \multirow{3}{*}{ Spectrum } & \multirow{3}{*}{$\begin{array}{c}\text { Zeeman } \\
\text { type }\end{array}$} & \multirow{3}{*}{$\begin{array}{c}\text { Wave- } \\
\text { length } \\
\text { in air }\end{array}$} & Inte & nsity & & \\
\hline & Meggers & Thompson & & & & Meggers & Thompson & & & & Meggers & Thompson & opectrom & type \\
\hline & lamp & lamp & & & & lamp & Iamp & & & & lamp & lamp & & \\
\hline 3337.581 & & 1 & II & & 3313.763 & 5 & 40 & II & 7 & 3285.35 & & 1 & II & \\
\hline 3337.420 & 5 & 20 & II & 5 & 3313.039 & & 3 & II & & 3285.168 & & 2 & II & \\
\hline 3337.171 & $30 \mathrm{r}$ & 100 & II & 7 & 3312.450 & 1 & 20 & II & & 3283.494 & 4 & 40 & II & 4 \\
\hline 3337.005 & & $5 \mathrm{~h}$ & II & & 3311.742 & 2 & 10 & II & & 3282.33 & 3 & & I & \\
\hline 3336.88 & & 1 & II & & 3311.630 & 3 & 8 & II & & 3282.03 & & 5 & II & \\
\hline 3336.718 & & 4 & II & & 3311.180 & & 2 & II & & 3281.715 & 1 & 7 & II & \\
\hline 3336.68 & 20 & 1 & I & & 3311.050 & 4 & 30 & II & & 3280.850 & & 4 & II & \\
\hline 3335.826 & 1 & 10 & II & & 3310.937 & 10 & 80 & II & 7 & 3280.335 & 1 & 10 & II & \\
\hline 3335.435 & & 2 & II & & 3310.815 & & 1 & II & & 3279.89 & & 4 & II & \\
\hline 3335.327 & & 3 & II & & 3310.20 & & 1 & II & & 3279.32 & & 4 & II & \\
\hline 3334.321 & 1 & 3 & II & & 3309.512 & 10 & 70 & II & & 3278.99 & & 1 & II & \\
\hline 3333.800 & & 4 & II & & 3309.470 & 2 & 20 & II & & 3278.614 & 50 & 1 & I & \\
\hline 3333.057 & 200 & 1000 & II & 4 & 3309.372 & 70 & 300 & II & 5 & 3278.56 & 5 & & I & \\
\hline 3332.774 & & 7 & II & & 3309.185 & 5 & 50 & II & & 3278.400 & 2 & 20 & II & 4 \\
\hline 3332.150 & 9 & & I & & 3308.461 & & 2 & II & & 3278.057 & 7 & 70 & II & 6 \\
\hline 3331.95 & 1 & & I & & 3308.210 & & 1 & II & & 3277.73 & & 4 & II & \\
\hline 3331.220 & 5 & 50 & II & 4 & 3306.783 & 100 & 400 & II & 7 & 3277.607 & 4 & 15 & II & \\
\hline 3330.238 & & 1 & II & & 3305.733 & $500 \mathrm{r}$ & 2000 & II & 6 & 3277.575 & & 1 & II & \\
\hline 3329.93 & 4 & & I & & 3305.252 & 20 & 20 & $\mathrm{I}, \mathrm{II}$ & & 3277.07 & & 1 & II & \\
\hline 3329.50 & 3 & 5 & II & & 3304.770 & 70 & 400 & II & 6 & 3276.218 & 15 & & I & \\
\hline 3329.382 & 50 & 100 & II & 6 & 3304.558 & 80 & 400 & II & 5 & 3275.801 & 50 & 200 & II & 6 \\
\hline 3329.087 & & 4 & II & & 3304.346 & & 6 & II & & 3275.19 & & 7 & II & \\
\hline 3329.017 & 3 & 10 & II & & 3303.926 & & 1 & II & & 3275.14 & & 1 & II & \\
\hline 3328.584 & & 8 & II & & 3303.864 & & 2 & II & & 3275.01 & 1 & 8 & II & \\
\hline 3328.51 & 2 & & I & & 3303.449 & & 1 & II & & 3274.96 & & 2 & II & \\
\hline 3328.39 & & 1 & II & & 3303.203 & & 2 & II & & 3274.770 & 6 & & I & \\
\hline 3327.756 & 8 & 80 & II & 6 & 3302.978 & 3 & & I & & 3273.940 & 7 & 70 & II & \\
\hline 3327.485 & 1 & 15 & II & & 3302.368 & 7 & & I & & 3273.65 & & 1 & II & \\
\hline 3327.195 & 3 & 3 & I,II & & 3302.202 & & 1 & II & & 3272.98 & & 2 & II & \\
\hline 3326.949 & 2 & 20 & II & 5 & 3301.780 & 1 & 10 & II & & 3272.644 & 30 & & I & \\
\hline 3326.745 & & 4 & II & & 3301.711 & 10 & 20 & II & 6 & 3271.528 & 20 & 150 & II & \\
\hline 3326.266 & 40 & 200 & II & 5 & 3301.481 & 1 & & I & & 3271.247 & 2 & 20 & II & \\
\hline 3325.512 & 1 & 2000 & III & 3 & 3301.328 & & 1 & II & & 3271.190 & 10 & 40 & II & \\
\hline 3325.104 & 9 & 50 & II & 4 & 3299.828 & 200 & 10 & I & 7 & 3271.125 & 3 & 30 & II & \\
\hline 3324.80 & & 2 & II & & 3299.647 & 2 & & I & & 3270.57 & & 2 & II & \\
\hline 3324.470 & 10 & 70 & II & 4 & 3299.447 & & 1 & II & & 3269.265 & 50 & 200 & II & \\
\hline 3324.172 & 40 & 300 & II & 5 & 3299.400 & 1 & 6 & II & & 3268.94 & & 2 & II & \\
\hline 3324.043 & 2 & 12 & II & & 3298.896 & & 8 & II & & 3268.84 & & 1 & II & \\
\hline 3323.46 & & $3 \mathrm{~h}$ & II & & 3298.826 & 1 & 10 & II & & 3268.811 & 2 & & I & \\
\hline 3322.963 & 20 & 250 & II & 5 & 3298.102 & & 2 & II & & 3267.890 & 150 & 2 & I & \\
\hline 3322.87 & 1 & 2 & II & & 3297.851 & 10 & 100 & II & 6 & 3267.313 & 10 & & I & \\
\hline 3322.58 & & 1 & II & & 3297.486 & 8 & & I & & 3266.915 & & 6 & II & \\
\hline 3320.802 & & 1 & II & & 3296.501 & & 4 & II & & 3266.39 & & $4 \mathrm{~d}$ & II & \\
\hline 3320.642 & & 2 & II & & 3295.825 & & 4 & II & & 3266.163 & & 4 & II & \\
\hline 3320.314 & 10 & 200 & II & 5 & 3294.58 & & 2 & II & & 3265.930 & 3 & 30 & II & \\
\hline 3320.032 & 5 & 30 & II & & 3294.341 & 7 & 100 & II & 6 & 3265.247 & 2 & & I & \\
\hline 3319.737 & & 2 & II & & 3293.836 & & 2 & II & & 3265.068 & 7 & 60 & II & \\
\hline 3319.674 & 5 & & I & & 3293.685 & 7 & & I & & 3264.889 & 8 & 80 & II & \\
\hline 3319.412 & $700 \mathrm{r}$ & 50 & I & 7 & 3293.66 & & 3 & II & & 3264.114 & & 4 & II & \\
\hline 3319.180 & 80 & 500 & II & 5 & 3293.25 & & 1 & II & & 3263.938 & 1 & 10 & II & \\
\hline 3318.973 & 50 & 100 & II & 5 & 3293.19 & 10 & & I & & 3263.79 & & 1 & II & \\
\hline 3318.590 & & 1 & II & & 3292.92 & & 1 & II & & 3262.296 & 6 & 60 & II & \\
\hline 3318.283 & 3 & 40 & II & 5 & 3292.26 & 20 & & I & & 3261.759 & 5 & 50 & II & \\
\hline 3317.939 & 2 & 30 & II & & 3292.089 & 5 & 20 & II & & 3261.686 & 20 & 200 & II & 6 \\
\hline 3317.094 & & 3 & II & & 3291.967 & & 10 & II & & 3261.508 & 500 & 1500 & II & 5 \\
\hline 3316.834 & 10 & 200 & II & 4 & 3291.946 & 20 & & I & & 3261.02 & & 1 & II & \\
\hline 3316.496 & 300 & 30 & I & 1 & 3291.028 & & 4 & II & & 3260.82 & & 5 & II & \\
\hline 3315.390 & 20 & 200 & II & 5 & 3290.554 & & 8 & II & & 3259.99 & 1 & 8 & II & \\
\hline 3315.101 & $1 \mathrm{~h}$ & $15 \mathrm{~h}$ & II & & 3290.14 & & 4 & II & & 3259.100 & 100 & 500 & II & 6 \\
\hline 3314.720 & 10 & 20 & II & & 3289.370 & $60000 \mathrm{~A}$ & 50000 & II & 4 & 3258.663 & 1 & 10 & II & \\
\hline 3314.533 & 20 & 5 & I & & 3287.81 & & 1 & II & & 3258.453 & & 8 & II & \\
\hline 3314.52 & & 10 & II & & 3286.960 & 10 & 70 & II & 5 & 3256.86 & & 1 & II & \\
\hline $33 \mathrm{i} 4.27$ & 1 & & I & & 3285.78 & & 1 & II & & 3256.030 & 5 & 50 & II & \\
\hline
\end{tabular}


TABLE 1.-Emission spectra of ytterbium-Continued

\begin{tabular}{|c|c|c|c|c|c|c|c|c|c|c|c|c|c|c|}
\hline \multirow{3}{*}{$\begin{array}{c}\begin{array}{c}\text { Wave- } \\
\text { length } \\
\text { in air }\end{array} \\
\AA\end{array}$} & \multicolumn{2}{|c|}{ Intensity } & \multirow{3}{*}{ Spectrum } & \multirow{3}{*}{$\begin{array}{c}\text { Zeeman } \\
\text { type }\end{array}$} & \multirow{3}{*}{$\begin{array}{c}\text { Wave- } \\
\text { length } \\
\text { in air }\end{array}$} & \multicolumn{2}{|c|}{ Intensity } & \multirow{3}{*}{ Spectrum } & \multirow{3}{*}{$\begin{array}{c}\text { Zeeman } \\
\text { type }\end{array}$} & \multirow{3}{*}{$\begin{array}{c}\begin{array}{c}\text { Wave- } \\
\text { length } \\
\text { in air }\end{array} \\
\AA \\
\end{array}$} & & nsity & & \\
\hline & Meggers & Thompson & & & & Meggers & Thompson & & & & Meggers & Thompson & spectrum & type \\
\hline & lamp & lamp & & & & lamp & lamp & & & & lamp & lamp & & \\
\hline 3255.24 & & 1 & II & & 3223.854 & 4 & 20 & II & & 3188.26 & & 1 & II & \\
\hline 3254.62 & & 2 & II & & 3223.105 & 4 & 40 & II & & 3187.51 & & 2 & II & \\
\hline 3254.197 & 100 & 300 & II & & 3222.696 & 150 & 3 & I & & 3187.01 & & 3 & II & \\
\hline 3253.911 & 10 & 100 & II & & 3222.55 & & 1 & II & & 3186.580 & 10 & 100 & II & 4 \\
\hline 3253.545 & 20 & 1 & I & & 3222.11 & & 5 & II & & 3185.64 & & 1 & II & \\
\hline 3253.340 & 20 & 120 & II & & 3221.486 & 10 & 70 & II & 5 & 3185.03 & & 1 & II & \\
\hline 3253.023 & 3 & & I & & 3221.246 & 15 & 150 & II & 4 & 3184.71 & & 1 & II & \\
\hline 3252.106 & 1 & 10 & II & & 3220.96 & & 2 & II & & 3183.90 & 3 & & I & \\
\hline 3250.505 & 1 & 5 & II & & 3220.814 & & 8 & II & & 3183.498 & 1 & 5 & II & \\
\hline 3249.80 & & 1 & II & & 3219.633 & & 8 & II & & 3183.46 & 1 & 5 & II & - \\
\hline 3249.57 & & 1 & II & & 3219.408 & 2 & 9 & II & & 3183.07 & 2 & & I & \\
\hline 3249.17 & & 4 & II & & 3219.325 & 1 & 10 & II & & 3182.86 & & 4 & II & \\
\hline 3248.446 & 2 & 10 & II & & 3218.913 & 2 & 15 & II & & 3182.52 & 1 & 8 & II & \\
\hline 3247.932 & 1 & 5 & II & & 3218.323 & 100 & 600 & II & 7 & 3182.165 & 2 & 10 & II & \\
\hline 3246.85 & & 1 & II & & 3217.96 & & 1 & II & & 3182.09 & 1 & & I & \\
\hline 3246.657 & 1 & 7 & II & & 3217.66 & & 3 & II & & 3181.64 & & 1 & II & \\
\hline 3246.28 & & 1 & II & & 3217.45 & & 1 & II & & 3181.27 & 1 & 4 & II & \\
\hline 3246.060 & & 10 & II & & 3217.40 & 5 & & I & & 3181.03 & 1 & 9 & II & \\
\hline 3245.70 & & 5 & II & & 3217.29 & 1 & 7 & II & & 3180.919 & 200 & 800 & II & 5 \\
\hline 3245.05 & & 6 & II & & 3217.176 & 200 & 1000 & II & 5 & 3180.48 & & 1 & II & \\
\hline 3244.46 & 2 & & I & & 3216.66 & 2 & & I & & 3179.94 & 1 & 10 & II & \\
\hline 3243.46 & & 5 & II & & 3216.273 & & 50 & III & & 3179.344 & 30 & 70 & II & \\
\hline 3243.00 & 4 & 30 & II & & 3215.927 & 10 & 100 & II & & 3178.85 & & 1 & II & \\
\hline 3242.12 & 1 & 10 & II & & 3215.68 & & 2 & II & & 3177.815 & & 1 & II & \\
\hline 3242.048 & 4 & 50 & II & & 3215.456 & 10 & 60 & II & & 3177.78 & & 2 & II & \\
\hline 3241.86 & 30 & 1 & I & & 3215.38 & & 2 & II & & 3176.26 & & 3 & II & \\
\hline 3240.946 & 20 & 1 & I & & 3214.330 & 2 & 20 & II & & 3176.06 & & 1 & II & \\
\hline 3240.57 & & 3 & III & & 3214.14 & & 1 & II & & 3175.759 & 25 & 150 & II & 6 \\
\hline 3239.580 & 200 & 5 & I & & 3213.172 & & 6 & II & & 3174.22 & & 2 & II & \\
\hline 3239.39 & 1 & 8 & II & & 3212.485 & & 5 & II & & 3173.797 & 20 & 150 & II & 7 \\
\hline 3239.195 & 50 & 200 & II & 6 & 3210.84 & & 7 & II & & 3171.176 & 100 & 300 & II & 6 \\
\hline 3237.888 & 10 & & I & & 3210.112 & 20 & 200 & II & 5 & 3169.558 & 10 & & I & \\
\hline 3237.215 & & 5 & II & & 3208.01 & & 3 & II & & 3169.056 & 200 & 1200 & II & 5 \\
\hline 3236.488 & 20 & 60 & II & & 3207.870 & 2 & & I & & 3168.985 & 3 & 10 & II & \\
\hline 3236.24 & 10 & & I & & 3207.702 & 4 & 30 & II & 5 & 3168.405 & 8 & 60 & II & 5 \\
\hline 3236.160 & 40 & 300 & II & 4 & 3207.538 & 2 & 10 & II & & 3167.72 & & 1 & II & \\
\hline 3235.975 & 3 & 30 & II & & 3207.40 & & 1 & II & & 3167.044 & 1 & 8 & II & \\
\hline 3235.858 & & 6 & II & & 3207.380 & 6 & & I & & 3166.727 & 2 & 20 & II & \\
\hline 3235.635 & 2 & & I & & 3206.148 & 20 & 200 & II & 7 & 3166.644 & 10 & & I & \\
\hline 3235.61 & & 8 & II & & 3205.863 & 20 & 50 & II & & 3166.407 & 10 & & I & \\
\hline 3235.520 & 4 & & I & & 3205.58 & & 1 & II & & 3165.981 & 1 & 5 & II & \\
\hline 3234.740 & 2 & 10 & II & & 3204.681 & 20 & 200 & II & 4 & 3165.875 & & 7 & II & \\
\hline 3233.487 & 3 & 40 & II & & 3203.989 & 1 & 10 & II & & 3165.206 & 60 & 500 & II & 4 \\
\hline 3233.38 & & 1 & II & & 3202.76 & & 2 & II & & 3164.973 & 6 & 15 & II & \\
\hline 3232.29 & & 4 & II & & 3201.35 & 5 & 30 & II & & 3163.985 & 4 & & I & \\
\hline 3231.986 & 50 & 300 & II & 6 & 3201.160 & $800 \mathrm{r}$ & 2000 & II & 5 & 3163.796 & 100 & 600 & II & 7 \\
\hline 3231.90 & 1 & 10 & II & & 3200.69 & & 1 & II & & 3162.737 & 4 & & I & \\
\hline 3231.81 & & 10 & II & & 3199.785 & 5 & 50 & II & & 3162.293 & 50 & 5 & I & \\
\hline 3231.376 & 10 & 50 & II & & 3199.24 & & 1 & II & & 3161.80 & & 2 & II & \\
\hline 3231.228 & & 2 & II & & 3198.647 & 400 & 1000 & II & 5 & 3161.55 & 2 & & I & \\
\hline 3229.93 & 2 & & I & & 3196.354 & 10 & 50 & II & 7 & 3160.894 & 1 & 8 & II & \\
\hline 3229.814 & 50 & 150 & II & 6 & 3196.052 & 2 & 20 & II & & 3160.12 & & 1 & II & \\
\hline 3229.475 & 2 & 20 & II & & 3195.585 & 20 & 200 & II & 5 & 3160.01 & & 3 & II & \\
\hline 3228.575 & & 2000 & III & & 3195.349 & 15 & & I & & 3159.79 & & 2 & II & \\
\hline 3228.503 & 5 & 40 & II & & 3194.765 & 10 & 100 & II & 7 & 3159.59 & & 1 & II & \\
\hline 3227.478 & 100 & 2 & I & & 3194.242 & 8 & 70 & II & 6 & 3159.49 & & 2 & II & \\
\hline 3226.725 & 20 & 80 & II & 4 & 3192.885 & $1000 \mathrm{r}$ & 3000 & II & 7 & 3159.34 & & 1 & II & \\
\hline 3225.885 & 100 & 400 & II & 6 & 3192.62 & 4 & 30 & II & & 3159.04 & & 2 & II & \\
\hline $\begin{array}{l}3225.78 \\
325.78\end{array}$ & 20 & 200 & II & 7 & 3192.45 & 1 & 8 & II & & 3158.869 & 5 & 10 & II & \\
\hline 3224.890 & & 4 & II & & 3192.15 & & 5 & II & & 3158.43 & 1 & 7 & II & \\
\hline 3224.752 & & 3 & II & & 3191.352 & & 800 & III & 1 & 3158.300 & 40 & 300 & II & 5 \\
\hline 3224.43 & & 4 & II & & 3190.780 & 8 & 30 & II & & 3158.145 & 1 & & I & \\
\hline 3224.25 & & 1 & II & & 3190.58 & & 5 & II & & 3157.043 & & 7 & II & \\
\hline
\end{tabular}


TABLE 1.-Emission spectra of ytterbium-Continued

\begin{tabular}{|c|c|c|c|c|c|c|c|c|c|c|c|c|c|c|}
\hline \multirow{3}{*}{$\begin{array}{c}\text { Wave- } \\
\text { length } \\
\text { in air }\end{array}$} & \multicolumn{2}{|c|}{ Intensity } & \multirow{3}{*}{ Spectrum } & \multirow{3}{*}{$\begin{array}{c}\text { Zeeman } \\
\text { type }\end{array}$} & \multirow{3}{*}{$\begin{array}{c}\text { Wave- } \\
\text { length } \\
\text { in air }\end{array}$} & \multicolumn{2}{|c|}{ Intensity } & \multirow{3}{*}{ Spectrum } & \multirow{3}{*}{$\begin{array}{c}\text { Zeeman } \\
\text { type }\end{array}$} & \multirow{3}{*}{$\begin{array}{c}\text { Wave- } \\
\text { length } \\
\text { in air }\end{array}$} & & ensity & & \\
\hline & Meggers & Thompson & & & & Meggers & Thompson & & & & Meggers & Thompson & Jpecinom & type \\
\hline & lamp & lamp & & & & lamp & lamp & & & & lamp & $\operatorname{lamp}$ & & \\
\hline 3156.365 & & 4 & II & & 3126.012 & 5 & 4000 & III & 7 & 3092.496 & 5 & 3000 & III & 3 \\
\hline 3155.798 & 30 & 40 & II & & 3125.885 & 1 & 10 & II & & 3092.10 & 1 & 8 & II & \\
\hline 3155.52 & & 2 & II & & 3125.700 & 5 & 20 & II & & 3091.10 & 1 & 6 & II & \\
\hline 3155.284 & 5 & 50 & II & & 3125.443 & 50 & 100 & II & & 3090.924 & 6 & 15 & II & \\
\hline 3155.183 & 40 & 200 & II & 6 & 3124.25 & & 2 & II & & 3090.747 & 1 & 10 & II & \\
\hline 3154.194 & 5 & 40 & II & & 3123.870 & & 6 & II & & 3089.61 & 3 & 20 & II & \\
\hline 3153.880 & 100 & 700 & II & 7 & 3123.510 & 8 & 60 & II & & 3089.102 & $150 \mathrm{r}$ & 500 & II & 4 \\
\hline 3153.616 & 20 & 100 & II & & 3122.74 & & 1 & II & & 3089.04 & 10 & & I & \\
\hline 3153.180 & 40 & 300 & II & 5 & 3122.48 & & 3 & II & & 3088.823 & 3 & 30 & II & \\
\hline 3152.880 & 2 & & I & & 3122.185 & 2 & 20 & II & & 3088.78 & 1 & 9 & II & \\
\hline 3152.44 & 4 & 40 & II & & 3121.38 & & 2 & II & & 3088.66 & & 3 & II & \\
\hline 3151.90 & & 1 & II & & 3119.73 & & 1 & II & & 3087.998 & 1 & 15 & II & \\
\hline 3151.436 & 15 & 100 & II & & 3119.37 & & 2 & II & & 3087.720 & & 6 & II & \\
\hline 3151.255 & 3 & .8 & II & & 3119.10 & & 1 & II & & 3087.46 & & 2 & II & \\
\hline 3150.46 & 3 & 30 & II & & 3117.806 & $1000 \mathrm{r}$ & 3000 & II & 6 & 3086.971 & 10 & 100 & II & 4 \\
\hline 3150.19 & & 6 & II & & 3117.318 & 2 & & I & & 3086.508 & 8 & 40 & II & \\
\hline 3150.12 & & 2 & II & & 3116.702 & 60 & 300 & II & 5 & 3085.817 & 10 & 70 & II & 6 \\
\hline 3148.998 & 30 & 200 & II & & 3116.487 & 10 & 70 & II & & 3085.434 & 9 & 60 & II & \\
\hline 3148.43 & & 2 & II & & 3116.342 & 10 & 40 & II & & 3085.21 & 8 & & I & \\
\hline 3148.08 & & 1 & II & & 3116.070 & 15 & 70 & II & 6 & 3084.347 & 7 & 70 & II & 6 \\
\hline 3147.450 & 1 & & I & & 3115.340 & 100 & 400 & II & 7 & 3083.809 & & 3 & II & \\
\hline 3146.134 & 8 & 60 & II & & 3115.25 & 1 & 8 & II & & 3083.44 & & 1 & II & \\
\hline 3145.540 & 150 & 500 & II & & 3114.905 & 3 & 20 & II & & 3082.34 & & 1 & II & \\
\hline 3145.063 & 80 & 600 & II & 6 & 3114.69 & & 1 & II & & 3082.264 & & 6 & II & \\
\hline 3142.813 & 1 & 7 & II & & 3114.505 & & 4 & II & & 3082.05 & & 1 & II & \\
\hline 3142.74 & & 2 & II & & 3112.980 & 1 & 10 & II & & 3081.625 & & 8 & II & \\
\hline 3141.733 & 60 & 400 & II & 4 & 3112.91 & & 4 & II & & 3081.18 & & 1 & II & \\
\hline 3141.17 & & 3 & II & & 3112.742 & 5 & & I & & 3081.142 & 2 & & I & \\
\hline 3140.936 & $1000 \mathrm{r}$ & 2000 & II & 6 & 3111.047 & 1 & 6 & II & & 3080.552 & 3 & 30 & II & 6 \\
\hline 3140.28 & & 2 & II & & 3109.786 & 5 & 40 & II & & 3080.49 & & 3 & II & \\
\hline 3139.75 & 1 & 6 & II & & 3109.52 & & 2 & II & & 3080.17 & 3 & 20 & II & \\
\hline 3139.27 & & 2 & II & & 3109.10 & 1 & 5 & II & & 3079.593 & & 7 & II & \\
\hline 3138.577 & 1 & 1000 & III & & 3107.902 & $1000 \mathrm{r}$ & 3000 & II & 4 & 3079.264 & 7 & 1 & I & \\
\hline 3138.276 & & 5 & II & & 3107.760 & 100 & 500 & II & 5 & 3078.450 & 1 & 10 & II & \\
\hline 3138.07 & & 2 & II & & 3106.97 & & 2 & II & & 3078.31 & 1 & 10 & II & \\
\hline 3137.622 & & 10 & II & & 3106.94 & 1 & 6 & II & & 3077.82 & & 2 & II & \\
\hline 3137.51 & & 1 & II & & 3106.740 & 2 & 10 & II & & 3077.34 & & 7 & II & \\
\hline 3136.760 & 80 & 400 & II & 6 & 3106.133 & & 3 & II & & 3077.18 & & 8 & II & \\
\hline 3136.67 & 1 & 10 & II & & 3106.007 & & 4 & II & & 3077.14 & & 6 & II & \\
\hline 3136.18 & & 2 & II & & 3105.37 & & 1 & II & & 3076.50 & & 1 & II & \\
\hline 3135.989 & & 4 & II & & 3103.81 & & 4 & II & & 3076.013 & 30 & 150 & II & 6 \\
\hline 3135.93 & & 1 & II & & 3103.36 & & 1 & II & & 3075.10 & & 2 & II & \\
\hline 3135.43 & & 1 & II & & 3102.71 & & 2 & II & & 3074.92 & & 2 & II & \\
\hline 3135.316 & 1 & 10 & II & & 3102.18 & & 20 & III & & 3074.84 & & 3 & II & \\
\hline 3135.12 & 4 & 30 & II & & 3102.074 & 30 & 150 & II & 5 & 3074.63 & 3 & & I & \\
\hline 3133.96 & & 1 & II & & 3101.688 & & 5 & II & & 3074.60 & 2 & & I & \\
\hline 3133.845 & & 3 & II & & 3101.361 & 50 & 250 & II & 4 & 3074.50 & 1 & 10 & II & \\
\hline 3133.778 & & 3 & II & & 3101.004 & 15 & 100 & II & & 3073.89 & & 3 & II & \\
\hline 3132.628 & 15 & 150 & II & 6 & 3100.745 & 5 & & I & & 3073.680 & 20 & 100 & II & \\
\hline 3132.478 & 2 & 15 & II & & 3100.25 & & 4 & II & & 3073.19 & & 3 & II & \\
\hline 3132.294 & 1 & 8 & II & & 3100.03 & & 2 & II & & 3072.486 & 6 & 60 & II & \\
\hline 3131.397 & & 1 & II & & 3099.114 & 1 & 10 & II & & 3072.41 & & 4 & II & \\
\hline 3131.38 & & 3 & II & & 3098.850 & 4 & 10 & II & & 3071.583 & 5 & 50 & II & \\
\hline 3130.862 & 2 & & I & & 3098.384 & & 3 & II & & 3071.26 & & 1 & II & \\
\hline 3129.145 & 8 & 80 & II & 5 & 3097.331 & 1 & 8 & II & & 3070.98 & 1 & & I & \\
\hline 3128.60 & & 1 & II & & 3096.278 & & 4 & II & & 3070.28 & 2 & 7 & II & \\
\hline 3127.866 & 20 & 120 & II & 6 & 3096.10 & & 1 & II & & 3069.34 & & 2 & II & \\
\hline 3127.64 & & 2 & II & & 3095.415 & 30 & 60 & II & & 3068.69 & 1 & 10 & II & \\
\hline 3127.49 & & 2 & II & & 3095.221 & 10 & 60 & II & 6 & 3068.284 & 7 & 60 & II & \\
\hline 3127.31 & & $2 \mathrm{~h}$ & II & & 3094.894 & 100 & 200 & II & & 3067.986 & 2 & 10 & II & \\
\hline 3127.138 & 4 & 40 & II & & 3093.870 & $200 \mathrm{r}$ & 1000 & II & 5 & 3067.365 & 10 & 70 & II & 6 \\
\hline 3126.25 & 1 & 5 & II & & 3093.423 & 3 & 30 & II & 6 & 3066.54 & & 2 & II & \\
\hline 3126.192 & 4 & 20 & II & & 3093.40 & 1 & 10 & II & & 3066.487 & 2 & 6 & II & \\
\hline
\end{tabular}


TABLE 1.-Emission spectra of ytterbium-Continued

\begin{tabular}{|c|c|c|c|c|c|c|c|c|c|c|c|c|c|c|}
\hline \multirow{3}{*}{$\begin{array}{l}\text { Wave- } \\
\text { length } \\
\text { in air } \\
\AA \\
\end{array}$} & \multicolumn{2}{|c|}{ Intensity } & \multirow{3}{*}{ Spectrum } & \multirow{3}{*}{$\begin{array}{c}\text { Zeeman } \\
\text { type }\end{array}$} & \multirow{3}{*}{$\begin{array}{l}\text { Wave- } \\
\text { length } \\
\text { in air } \\
\AA \\
\end{array}$} & \multicolumn{2}{|c|}{ Intensity } & \multirow{3}{*}{ Spectrum } & \multirow{3}{*}{$\begin{array}{c}\text { Zeeman } \\
\text { type }\end{array}$} & \multirow{3}{*}{$\begin{array}{l}\text { Wave- } \\
\text { length } \\
\text { in air } \\
\AA \\
\end{array}$} & Inte & nsity & & \\
\hline & & Thompson & & & & & Thompson & & & & Meggers & Thompson & Jpectrom & type \\
\hline & lamp & lamp & & & & lamp & lamp & & & & lamp & lamp & & \\
\hline 3065.74 & & 7 & II & & 3039.276 & & 6 & II & & 3004.097 & 1 & 5 & II & \\
\hline 3065.52 & & 2 & II & & 3038.81 & & 2 & II & & 3003.60 & & 2 & II & \\
\hline 3065.48 & & 1 & II & & 3038.75 & 2 & 10 & II & & 3002.608 & 30 & 200 & II & \\
\hline 3065.040 & $300 \mathrm{r}$ & 1000 & II & 4 & 3038.531 & 5 & 30 & II & & 3002.47 & & 2 & II & \\
\hline 3064.868 & 30 & 150 & II & & 3037.992 & 15 & 90 & II & 6 & $3002: 035$ & 5 & 30 & II & 5 \\
\hline 3064.75 & & 2 & II & & 3036.820 & 10 & 60 & II & & 3001.864 & 2 & 10 & II & \\
\hline 3063.80 & & 4 & II & & 3036.13 & & 1 & II & & 3001.283 & 4 & 40 & II & 4 \\
\hline 3063.671 & 20 & 100 & II & & 3035.945 & 1 & 7 & II & & 3000.987 & 2 & 15 & II & \\
\hline 3063.350 & 2 & 10 & II & & 3034.99 & 1 & 5 & II & & 3000.865 & 2 & & I & \\
\hline 3063.125 & 40 & 200 & II & 5 & 3034.642 & 40 & 200 & II & 4 & 3000.465 & 100 & 300 & II & 4 \\
\hline 3062.524 & & 4 & II & & 3034.52 & & 2 & II & & 3000.13 & & 2 & II & \\
\hline 3062.06 & & 1 & II & & 3034.32 & & 1 & II & & 2999.642 & 5 & 10 & II & \\
\hline 3061.646 & & 6 & II & & 3033.860 & 30 & 100 & II & & 2999.43 & & 8 & III & \\
\hline 3060.440 & 5 & & I & & 3033.664 & 2 & 10 & II & & 2998.377 & 4 & 30 & II & \\
\hline 3060.394 & & 7 & II & & 3033.40 & & 2 & II & & 2998.000 & 1 & 800 & III & \\
\hline 3059.568 & & 7 & II & & 3033.28 & & 1 & II & & 2996.75 & & 2 & II & \\
\hline 3059.362 & & 4 & II & & 3033.14 & & 1 & II & & 2996.373 & & 5 & II & \\
\hline 3058.71 & & 2 & II & & 3031.625 & & 100 & III & & 2995.862 & 20 & 100 & II & 5 \\
\hline 3058.66 & & 5 & II & & 3031.110 & $5000 \mathrm{~A}$ & 3000 & II & 4 & 2995.03 & 6 & 30 & II & \\
\hline 3058.288 & & 4 & II & & 3029.486 & 2 & 2000 & III & 3 & 2994.94 & 2 & 20 & II & \\
\hline 3058.20 & & 1 & II & & 3028.38 & 2 & 20 & II & & 2994.805 & $150 \mathrm{r}$ & 600 & II & 6 \\
\hline 3057.29 & & 1 & II & & 3027.476 & 7 & & I & & 2994.53 & 2 & 20 & II & \\
\hline 3056.01 & & 2 & II & & 3026.669 & 100 & 700 & II & 4 & 2994.48 & 1 & 10 & II & \\
\hline 3055.60 & & 1 & II & & 3025.76 & & 2 & II & & 2993.939 & 10 & 70 & II & 6 \\
\hline 3055.46 & & 2 & II & & 3025.38 & & 6 & II & & 2992.28 & & 5 & II & \\
\hline 3055.350 & 5 & 15 & II & & 3024.935 & 2 & 15 & II & & 2991.872 & 40 & 200 & II & 5 \\
\hline 3055.157 & 10 & 60 & II & 4 & 3023.614 & 5 & 30 & II & 6 & 2991.70 & 5 & 9 & II & \\
\hline 3054.649 & 1 & 10 & II & & 3022.58 & & 1 & II & & 2991.33 & & 1 & II & \\
\hline 3053.928 & 2 & 20 & II & & 3022.454 & 10 & 60 & II & 7 & 2991.240 & 1 & 6 & II & \\
\hline 3053.452 & & 8 & II & & 3021.27 & & 1 & II & & 2990.366 & 30 & 200 & II & 5 \\
\hline 3053.084 & 1 & 10 & II & & 3020.99 & & 4 & II & & 2989.768 & 2 & 15 & II & \\
\hline 3052.20 & & 2 & II & & 3020.703 & 20 & 100 & II & 5 & 2989.376 & 6 & 10 & II & \\
\hline 3052.021 & 1 & 7 & II & & 3020.345 & 2 & 10 & II & & 2989.20 & 2 & 20 & II & 6 \\
\hline 3051.98 & & 2 & II & & 3020.211 & & 5 & II & & 2988.95 & & 5 & II & \\
\hline 3051.11 & & 2 & II & & 3019.440 & 2 & 15 & II & & 2980.56 & & 1 & II & \\
\hline 3050.64 & & 1 & II & & 3019.17 & & 2 & II & & 2988.30 & & 2 & II & \\
\hline 3050.50 & & 6 & II & & 3019.058 & 8 & 40 & II & & 2987.862 & 1 & 10 & II & \\
\hline 3049.84 & & 2 & II & & 3017.560 & 100 & 800 & II & 5 & 2987.25 & 1 & 6 & II & \\
\hline 3049.14 & & 7 & II & & 3016.95 & & 6 & II & & 2986.64 & & 2 & II & \\
\hline 3048.82 & & 2 & II & & 3016.40 & & 1 & II & & 2986.47 & & 1 & II & \\
\hline 3048.41 & & 6 & II & & 3014.97 & & 2 & II & & 2985.876 & 10 & 70 & II & 4 \\
\hline 3048.15 & & 2 & II & & 3014.526 & 10 & 60 & II & 7 & 2985.078 & 50 & 300 & II & 5 \\
\hline 3047.052 & 20 & 100 & II & 6 & 3014.427 & 40 & 200 & II & 5 & 2984.84 & 7 & 60 & II & \\
\hline 3046.482 & 30 & 200 & II & 4 & 3014.02 & & 8 & II & & 2984.44 & & 1 & II & \\
\hline 3046.27 & & 3 & II & & 3013.08 & & 2 & II & & 2983.990 & $200 \mathrm{r}$ & 1000 & II & 6 \\
\hline 3045.91 & & 2 & II & & 3012.80 & & 3 & II & & 2983.702 & 15 & 100 & II & \\
\hline 3045.84 & & 1 & II & & 3012.28 & & 1 & II & & 2983.369 & 4 & 8 & II & \\
\hline 3045.46 & & 4 & II & & 3011.842 & & 4 & II & & 2982.659 & 10 & 70 & II & 4 \\
\hline 3045.044 & 1 & 7 & II & & 3011.218 & 7 & & I & & 2982.494 & 20 & 150 & II & 4 \\
\hline 3044.83 & 10 & 60 & II & 6 & 3011.00 & & 1 & II & & 2982.055 & 2 & 8 & II & \\
\hline 3044.004 & 20 & 100 & II & 4 & 3010.735 & 2 & & I & & 2982.01 & & 5 & II & \\
\hline 3043.93 & & 4 & II & & 3010.625 & 50 & 300 & II & 4 & 2981.519 & 3 & 20 & II & \\
\hline 3043.07 & & 1 & II & & 3009.392 & 100 & 500 & II & 4 & 2980.482 & & 5 & II & \\
\hline 3042.78 & & 3 & II & & 3009.21 & 2 & & I & & 2979.859 & 10 & 60 & II & 4 \\
\hline 3042.650 & 50 & 300 & II & 4 & 3007.923 & 5 & 30 & II & & 2979.658 & 3 & 20 & II & 4 \\
\hline 3042.34 & & 2 & II & & 3007.56 & & 3 & II & & 2979.44 & & 1 & II & \\
\hline 3041.180 & 7 & & I & & 3006.863 & 10 & 70 & II & 5 & 2978.906 & 6 & 40 & II & 4 \\
\hline 3040.83 & & 2 & II & & 3006.30 & & 3 & II & & 2977.835 & & 50 & III & \\
\hline 3040.652 & & 30 & III & & 3006.14 & 5 & 40 & II & & 2977.76 & & 1 & II & \\
\hline 3040.504 & 5 & 30 & II & & 3005.766 & $800 \mathrm{r}$ & 3000 & II & 4 & 2977.695 & & 4 & II & \\
\hline 3040.444 & 2 & 15 & II & & 3005.118 & 5 & & I & & 2977.525 & 10 & 60 & II & 6 \\
\hline 3040.235 & 5 & 15 & II & & 3004.642 & 1 & 15 & II & & 2977.270 & 1 & 9 & II & \\
\hline 3039.665 & 40 & 300 & II & 4 & 3004.46 & 3 & $10 \mathrm{~h}$ & II & & 2976.53 & & 1 & II & \\
\hline
\end{tabular}


TABLE 1.-Emission spectra of ytterbium-Continued

\begin{tabular}{|c|c|c|c|c|c|c|c|c|c|c|c|c|c|c|}
\hline \multirow{3}{*}{$\begin{array}{c}\begin{array}{c}\text { Wave- } \\
\text { length } \\
\text { in air }\end{array} \\
\AA \\
\end{array}$} & \multicolumn{2}{|c|}{ Intensity } & \multirow{3}{*}{ Spectrum } & \multirow{3}{*}{$\begin{array}{c}\text { Zeeman } \\
\text { type }\end{array}$} & \multirow{3}{*}{$\begin{array}{c}\begin{array}{c}\text { Wave- } \\
\text { length } \\
\text { in air }\end{array} \\
\AA\end{array}$} & \multicolumn{2}{|c|}{ Intensity } & \multirow{3}{*}{ Spectrum } & \multirow{3}{*}{$\begin{array}{c}\text { Zeeman } \\
\text { type }\end{array}$} & \multirow{3}{*}{$\begin{array}{c}\text { Wave- } \\
\text { length } \\
\text { in air } \\
\AA \\
\end{array}$} & & ensity & & \\
\hline & Meggers & Thompson & & & & Meggers & Thompson & & & & Meggers & Thompson & Spectrum & type \\
\hline & lamp & lamp & & & & lamp & lamp & & & & lamp & lamp & & \\
\hline 2975.567 & 3 & 20 & II & & 2938.84 & 1 & 4 & II & & 2905.61 & & 3 & II & \\
\hline 2975.238 & & 6 & II & & 2938.51 & 3 & 20 & II & & 2904.97 & & 3 & II & \\
\hline 2973.36 & & 1 & II & & 2938.188 & 7 & 50 & II & 6 & 2904.79 & 1 & 7 & II & \\
\hline 2972.94 & & 6 & II & & 2937.186 & 15 & 100 & II & 5 & 2904.20 & & 1 & II & \\
\hline 2972.47 & 3 & 30 & II & 7 & 2935.52 & & 4 & II & & $2902: 924$ & 8 & 80 & II & 5 \\
\hline 2970.84 & 20 & 200 & II & 6 & 2935.245 & 1 & 7 & II & & 2902.41 & 6 & 40 & II & 6 \\
\hline 2970.564 & $4000 \mathrm{~A}$ & 3000 & II & 6 & 2935.108 & 30 & 200 & II & 5 & 2901.86 & & 1 & II & \\
\hline 2969.70 & 1 & 7 & II & & 2934.360 & 60 & 3 & I & & 2901.60 & & 1 & II & \\
\hline 2967.72 & & 4 & II & & 2933.82 & & 6 & III & & 2900.39 & & 2 & II & \\
\hline 2966.763 & 20 & 100 & II & 7 & 2932.90 & & 1 & II & & 2899.705 & 40 & 200 & II & 4 \\
\hline 2965.168 & 8 & 60 & II & & 2932.21 & & 1 & II & & 2899.236 & 5 & 40 & II & 5 \\
\hline 2964.82 & & 4 & II & & 2932.08 & & 2 & II & & 2898.30 & & 600 & III & 7 \\
\hline 2964.755 & $100 \mathrm{r}$ & 500 & II & 5 & 2931.27 & & 1 & II & & 2897.98 & & 2 & II & \\
\hline 2964.39 & 15 & 100 & II & 6 & 2929.70 & & 5 & II & & 2897.31 & & 4 & II & \\
\hline 2963.96 & & 4 & II & & 2929.48 & & 3 & II & & 2896.902 & 10 & 100 & II & 4 \\
\hline 2963.46 & 40 & 300 & II & 6 & 2929.44 & & 1 & II & & 2895.06 & 2 & 15 & II & \\
\hline 2963.255 & 10 & 70 & II & & 2928.973 & & 300 & III & & 2894.95 & 3 & 30 & II & 5 \\
\hline 2963.17 & 8 & 50 & II & & 2928.62 & 3 & & I & & 2893.620 & 10 & 100 & II & 4 \\
\hline 2962.522 & 40 & 250 & II & 6 & 2927.850 & 10 & 70 & II & 5 & 2891.384 & $3000 \mathrm{~A}$ & 2000 & II & 4 \\
\hline 2961.801 & 4 & 30 & II & & 2927.48 & 3 & & I & & 2891.16 & 4 & 40 & II & \\
\hline 2961.150 & 1 & 6 & II & & 2927.42 & & 1 & II & & 2890.28 & 1 & 8 & II & \\
\hline 2960.850 & 10 & 60 & II & 4 & 2927.116 & 1 & 10 & II & & 2888.24 & 15 & & I & \\
\hline 2958.425 & & 3 & II & & 2926.65 & 2 & 15 & II & & 2888.04 & $100 \mathrm{r}$ & 300 & II & 6 \\
\hline 2957.632 & 40 & 150 & II & & 2926.28 & & 1 & II & & 2887.74 & 2 & 7 & II & \\
\hline 2957.05 & & 3 & II & & 2924.65 & 2 & 9 & II & & 2886.263 & 30 & 200 & II & 5 \\
\hline 2955.61 & & 2 & II & & 2924.560 & & 4 & II & & 2885.97 & 10 & 70 & II & 4 \\
\hline 2955.318 & 40 & 200 & II & 5 & 2924.235 & 20 & 100 & II & 4 & 2885.594 & & 7 & II & \\
\hline 2955.09 & 1 & 4 & II & & 2922.35 & 2 & 7 & II & & 2885.02 & 1 & 8 & II & \\
\hline 2954.45 & & 1 & II & & 2922.235 & 4 & . & I & & 2884.51 & & 2 & II & \\
\hline 2953.016 & 5 & 40 & II & 4 & 2921.85 & & 1 & II & & 2883.87 & 1 & 10 & II & 6 \\
\hline 2952.55 & 4 & & I & & 2921.64 & & 2 & II & & 2883.686 & 10 & 1 & I & \\
\hline 2952.475 & & 4 & II & & 2921.119 & 40 & 200 & II & 6 & 2883.01 & & 6 & II & \\
\hline 2952.253 & 1 & 10 & II & & 2920.16 & 1 & 10 & II & & 2882.155 & 7 & 50 & II & 7 \\
\hline 2951.716 & 1 & 8 & II & & 2919.346 & $400 \mathrm{r}$ & 1000 & II & 6 & 2882.01 & & 1 & II & \\
\hline 2951.406 & 5 & 40 & II & & 2919.08 & & 4 & III & & 2881.93 & 7 & 60 & II & 5 \\
\hline 2951.022 & 8 & 50 & II & & 2918.89 & & 2 & II & & 2881.10 & & 6 & II & \\
\hline 2950.80 & 2 & 8 & II & & 2918.53 & & 1 & II & & 2879.83 & & 1 & II & \\
\hline 2950.64 & & 1 & II & & 2916.83 & 2 & 10 & II & & 2879.159 & 10 & 70 & II & 5 \\
\hline 2950.52 & 2 & 20 & II & & 2916.61 & 7 & 30 & II & & 2876.45 & & 1 & II & \\
\hline 2950.326 & 20 & 150 & II & 4 & 2916.431 & 10 & 70 & II & 4 & 2875.86 & & 400 & III & 7 \\
\hline 2949.840 & & 5 & II & & 2915.99 & & 4 & II & & 2874.83 & & 1 & II & \\
\hline 2948.65 & & 2 & II & & 2915.275 & $150 \mathrm{r}$ & 300 & II & 4 & 2874.11 & & 6 & II & \\
\hline 2947.76 & & 3 & II & & 2915.14 & & 4 & II & & 2873.490 & 15 & 1 & I & \\
\hline 2947.131 & 4 & 30 & II & & 2914.84 & 1 & 8 & II & & 2873.14 & & 3 & II & \\
\hline 2946.95 & & 5 & II & & 2914.48 & 7 & 40 & II & 6 & 2872.82 & & 1 & II & \\
\hline 2946.852 & 2 & 20 & II & & 2914.210 & $100 \mathrm{r}$ & 600 & II & 4 & 2872.11 & 2 & 10 & II & \\
\hline 2946.765 & 10 & 70 & II & 5 & 2913.75 & & 3 & II & & 2871.712 & 2 & 10 & II & \\
\hline 2946.305 & 30 & 200 & II & 6 & 2913.12 & 1 & 10 & II & & 2870.57 & & 3 & II & \\
\hline 2945.907 & $100 \mathrm{r}$ & 600 & II & 5 & 2912.865 & 10 & 50 & II & 4 & 2870.51 & & 3 & II & \\
\hline 2945.22 & & 3 & II & & 2912.51 & & 5 & II & & 2870.061 & 20 & 100 & II & 5 \\
\hline 2944.465 & 5 & 30 & II & & 2912.25 & & 2 & II & & 2869.61 & & 1 & II & \\
\hline 2944.336 & 5 & & I & & 2911.61 & & 3 & II & & 2869.546 & 1 & 15 & II & \\
\hline 2944.19 & & 2 & II & & 2911.523 & 40 & 200 & II & 6 & 2868.536 & & 5 & II & \\
\hline 2943.20 & & 2 & II & & 2910.43 & & 2 & II & & 2867.90 & & 7 & II & \\
\hline 2943.15 & & 1 & II & & 2909.88 & & 4 & II & & 2867.58 & 5 & 15 & II & \\
\hline 2942.823 & 10 & 50 & II & 4 & 2909.48 & 30 & 150 & II & 5 & 2867.20 & 1 & 7 & II & \\
\hline 2942.60 & & 2 & II & & 2909.19 & 20 & 100 & II & 7 & 2867.065 & $100 \mathrm{r}$ & 500 & II & 4 \\
\hline 2942.038 & 15 & 80 & II & 6 & 2908.55 & 1 & 9 & II & & 2866.179 & 4 & 30 & II & 6 \\
\hline 2941.77 & 1 & $\begin{array}{r}\text { ou } \\
4\end{array}$ & II & & 2908.33 & 10 & 50 & II & 4 & 2865.96 & 1 & 6 & II & \\
\hline 2941.11 & & 1 & II & & 2908.15 & & 4 & II & & 2865.74 & & 1 & II & \\
\hline 2940.517 & 25 & 150 & II & 5 & 2908.09 & 5 & 40 & II & & 2865.60 & 1 & 7 & II & \\
\hline 2940.25 & & 2 & II & & 2906.88 & 7 & 60 & II & 6 & 2865.328 & 5 & 30 & II & 6 \\
\hline 2939.534 & 20 & 100 & II & 5 & 2906.313 & 1 & 1000 & III & 3 & 2864.725 & 4 & 40 & II & 4 \\
\hline
\end{tabular}


TABLE 1.-Emission spectra of ytterbium-Continued

\begin{tabular}{|c|c|c|c|c|c|c|c|c|c|c|c|c|c|c|}
\hline \multirow{3}{*}{$\begin{array}{c}\begin{array}{c}\text { Wave- } \\
\text { length } \\
\text { in air }\end{array} \\
\AA \\
\end{array}$} & \multicolumn{2}{|c|}{ Intensity } & \multirow{3}{*}{ Spectrum } & \multirow{3}{*}{$\begin{array}{c}\text { Zeeman } \\
\text { type }\end{array}$} & \multirow{3}{*}{$\begin{array}{c}\begin{array}{c}\text { Wave- } \\
\text { length } \\
\text { in air }\end{array} \\
\AA \\
\end{array}$} & \multicolumn{2}{|c|}{ Intensity } & \multirow{3}{*}{ Spectrum } & \multirow{3}{*}{$\begin{array}{c}\text { Zeeman } \\
\text { type }\end{array}$} & \multirow{3}{*}{$\begin{array}{c}\begin{array}{c}\text { Wave- } \\
\text { length } \\
\text { in air }\end{array} \\
\AA \\
\end{array}$} & Inte & nsity & & \\
\hline & Meggers & Thompson & & & & Meggers & Thompson & & & & Meggers & Thompson & opecinom & type \\
\hline & lamp & lamp & & & & lamp & lamp & & & & lamp & lamp & & \\
\hline 2864.62 & 1 & 10 & II & & 2835.95 & & 1 & II & & 2800.006 & 8 & 50 & II & \\
\hline 2864.19 & & 1 & II & & 2835.48 & & 2 & II & & 2799.376 & 30 & 40 & II & 5 \\
\hline 2863.13 & 5 & 30 & II & & 2835.13 & & 1 & II & & 2798.211 & 20 & 100 & II & 6 \\
\hline 2862.97 & 2 & 5 & II & & 2834.972 & 10 & 70 & II & 6 & 2797.796 & 10 & 80 & II & 5 \\
\hline 2862.85 & 1 & 8 & II & & 2834.63 & & 4 & II & & 2797.28 & & 2 & II & \\
\hline 2861.34 & 20 & 200 & II & 6 & 2833.68 & & 1 & II & & 2796.764 & & 2 & II & \\
\hline 2861.212 & 40 & 300 & II & 5 & 2832.201 & 4 & 20 & II & 4 & 2795.60 & 5 & 600 & III & 1 \\
\hline 2861.11 & 5 & 30 & II & & 2830.989 & $30 \mathrm{r}$ & 200 & II & 5 & 2795.292 & 6 & 20 & II & 6 \\
\hline 2860.70 & 3 & 15 & II & & 2830.88 & 4 & 30 & II & 7 & 2795.074 & 8 & 40 & II & 4 \\
\hline 2860.393 & 20 & 100 & II & 7 & 2830.639 & 1 & 8 & II & & 2794.774 & 7 & 40 & II & 7 \\
\hline 2859.94 & 4 & 20 & II & & 2828.48 & 1 & 10 & II & & 2794.441 & 10 & 70 & II & 5 \\
\hline 2859.805 & $1000 \mathrm{R}$ & 1000 & II & 4 & 2827.921 & 3 & 20 & II & 7 & 2794.08 & 2 & 10 & II & \\
\hline 2859.392 & 30 & 200 & II & 6 & 2827.81 & 1 & 5 & II & & 2793.280 & 10 & 60 & II & 4 \\
\hline 2858.460 & 8 & 40 & II & 4 & 2826.735 & & 3 & II & & 2792.54 & & 3 & II & \\
\hline 2858.332 & 15 & 60 & II & 6 & 2826.522 & & 2 & II & & 2790.47 & & 3 & III & \\
\hline 2857.75 & & 2 & II & & 2826.013 & & 15 & III & & 2789.665 & 3 & & I & \\
\hline 2857.29 & 4 & 20 & II & 5 & 2824.974 & 20 & 100 & II & 5 & 2789.431 & 3 & 15 & II & \\
\hline 2857.13 & & 3 & II & & 2824.86 & 1 & 4 & II & & 2788.245 & & 300 & III & \\
\hline 2855.85 & 1 & 7 & II & & 2824.54 & 2 & 9 & II & & 2787.965 & 7 & 30 & II & 5 \\
\hline 2854.490 & 10 & 50 & II & 4 & 2824.20 & 2 & 10 & II & & 2787.010 & 1 & 7 & II & \\
\hline 2854.144 & 10 & 60 & II & 4 & 2823.95 & & 1 & III & & 2786.025 & 1 & 5 & II & \\
\hline 2854.04 & 4 & 40 & II & & 2823.585 & 7 & 50 & II & 7 & 2785.903 & 2 & & I & \\
\hline 2853.68 & 3 & 30 & II & 4 & 2823.320 & & 4 & II & & 2785.750 & 8 & 30 & II & 5 \\
\hline 2853.409 & 5 & 60 & II & 6 & 2821.52 & & 3 & II & & 2785.066 & & 2 & II & \\
\hline 2852.63 & & 1 & II & & 2821.152 & $30 \mathrm{r}$ & 200 & II & 4 & 2784.966 & 4 & & I & \\
\hline 2851.92 & & 4 & II & & 2820.12 & & 3 & II & & 2784.656 & 30 & 150 & II & 6 \\
\hline 2851.86 & 10 & 70 & II & 5 & 2819.75 & & 2 & II & & 2783.16 & & 2 & II & \\
\hline 2851.126 & $500 \mathrm{R}$ & 1000 & II & 4 & 2819.49 & 2 & 10 & II & 6 & 2782.58 & & 3 & II & \\
\hline 2850.64 & & 3 & II & & 2819.24 & 2 & & I & & 2782.54 & 1 & 5 & II & \\
\hline 2850.13 & & 1 & II & & 2818.722 & 6 & 1000 & III & 1 & 2782.203 & 1 & 5 & II & \\
\hline 2849.90 & & 3 & II & & 2817.66 & & 2 & II & & 2782.102 & 1 & 2 & II & \\
\hline 2849.84 & & 1 & II & & 2816.915 & & 600 & III & 7 & 2781.493 & & 2 & II & \\
\hline 2849.71 & & 1 & III & & 2816.368 & 2 & 8 & II & & 2780.820 & 1 & 3 & II & \\
\hline 2849.336 & 6 & 40 & II & 6 & 2816.317 & 4 & 20 & II & & 2780.125 & & 3 & II & \\
\hline 2848.88 & 1 & 7 & II & & 2815.20 & & 1 & II & & 2780.032 & 2 & 15 & II & \\
\hline 2848.445 & 20 & 100 & II & 4 & 2814.87 & 2 & 10 & II & & 2779.412 & & 3 & III & \\
\hline 2847.97 & & 3 & II & & 2814.528 & 20 & 100 & II & 6 & 2776.688 & 1 & 5 & II & \\
\hline 2847.75 & & 2 & II & & 2814.241 & 2 & 10 & II & & 2776.280 & $60 \mathrm{r}$ & 200 & II & 6 \\
\hline 2847.64 & & 2 & II & & 2814.14 & 2 & & I & & 2775.440 & 2 & 10 & II & 6 \\
\hline 2847.28 & & 4 & II & & 2813.00 & & 1 & II & & 2774.304 & 5 & 20 & II & 7 \\
\hline 2847.175 & $400 \mathrm{r}$ & 1000 & II & 5 & 2812.612 & 2 & 6 & II & & 2773.91 & & 2 & II & \\
\hline 2847.03 & & 1 & II & & 2811.133 & 3 & 8 & II & & 2771.86 & & 2 & II & \\
\hline 2846.324 & & 5 & II & & 2810.723 & 5 & 20 & II & 4 & 2771.324 & 20 & 100 & II & 4 \\
\hline 2846.183 & 1 & 5 & II & & 2810.109 & 4 & 15 & II & & 2770.405 & & 2 & II & \\
\hline 2845.355 & & 4 & II & & 2809.326 & 2 & 9 & II & & 2769.412 & & 2 & II & \\
\hline 2844.685 & 4 & 20 & II & & 2808.75 & & 2 & II & & 2768.281 & 2 & 20 & II & \\
\hline 2844.067 & 4 & 10 & II & & 2808.514 & 2 & 50 & III & & 2766.624 & 2 & 7 & II & \\
\hline 2843.83 & 3 & 15 & II & & 2808.305 & 4 & 20 & II & 5 & 2766.167 & 1 & 3 & II & \\
\hline 2843.702 & 1 & 7 & II & & 2807.83 & 2 & 10 & II & & 2765.495 & & 100 & III & \\
\hline 2842.955 & & 300 & III & 7 & 2807.744 & 2 & 6 & II & & 2765.046 & 2 & 8 & II & \\
\hline 2842.586 & 6 & 30 & II & 6 & 2807.32 & 1 & 4 & II & & 2764.414 & 10 & 50 & II & 6 \\
\hline 2842.51 & 2 & 8 & II & & 2807.22 & & 10 & III & & 2762.716 & 1 & 3 & II & \\
\hline 2842.291 & 1 & 7 & II & & 2807.180 & 2 & 8 & II & & 2761.70 & & 4 & II & \\
\hline 2842.140 & 3 & 15 & II & 7 & 2806.43 & & 1 & II & & 2761.373 & 10 & 70 & II & 7 \\
\hline 2841.308 & 2 & 20 & II & 6 & 2806.057 & 1 & 4 & II & & 2760.780 & 10 & 70 & II & 5 \\
\hline 2841.26 & & 1 & II & & 2804.807 & 1 & 3 & II & & 2759.532 & 3 & 20 & II & \\
\hline 2841.02 & & 2 & II & & 2804.260 & 5 & 30 & II & 4 & 2759.257 & & 3 & II & \\
\hline 2839.91 & & 2 & II & & 2803.427 & 2 & 1000 & III & 7 & 2759.003 & 7 & 40 & II & 4 \\
\hline 2839.85 & & 1 & II & & 2803.319 & & 400 & III & 7 & 2758.263 & & 1 & II & \\
\hline 2839.17 & & 1 & II & & 2801.598 & 1 & 5 & II & & 2758.065 & & 1 & II & \\
\hline 2838.64 & 3 & 15 & II & & 2801.272 & & 1 & II & & 2757.542 & & 3 & II & \\
\hline 2837.92 & & 2 & II & & 2800.109 & 2 & 10 & II & & 2756.76 & & 200 & III & \\
\hline 2837.09 & & 1 & II & & 2800.060 & 6 & 30 & II & & 2755.94 & & 200 & III & \\
\hline
\end{tabular}


TABLE 1.-Emission spectra of ytterbium-Continued

\begin{tabular}{|c|c|c|c|c|c|c|c|c|c|c|c|c|c|c|}
\hline \multirow{3}{*}{$\begin{array}{l}\text { Wave- } \\
\text { length } \\
\text { in air }\end{array}$} & \multicolumn{2}{|c|}{ Intensity } & \multirow{3}{*}{ Spectrum } & \multirow{3}{*}{$\begin{array}{c}\text { Zeeman } \\
\text { type }\end{array}$} & \multirow{3}{*}{$\begin{array}{l}\text { Wave- } \\
\text { length } \\
\text { in air } \\
\circ \\
\text { A }\end{array}$} & \multicolumn{2}{|c|}{ Intensity } & \multirow{3}{*}{ Spectrum } & \multirow{3}{*}{$\begin{array}{l}\text { Zeeman } \\
\text { type }\end{array}$} & \multirow{3}{*}{$\begin{array}{c}\text { Wave- } \\
\text { length } \\
\text { in air } \\
\AA \\
\end{array}$} & Inte & ensity & & \\
\hline & Meggers & Thompson & & & & Meggers & Thompson & & & & Meggers & Thompson & Spectrum & type \\
\hline & lamp & lamp & & & & lamp & lamp & & & & lamp & lamp & & \\
\hline 2754.943 & 3 & 20 & II & 6 & 2719.005 & 3 & 15 & II & 7 & 2681.180 & & 2 & II & \\
\hline 2754.105 & & 2 & II & & 2718.349 & $40 \mathrm{r}$ & 200 & II & 7 & 2680.993 & & 2 & III & \\
\hline 2753.584 & 5 & 20 & II & & 2717.76 & & 2 & II & & 2680.404 & 6 & 40 & II & 6 \\
\hline 2751.452 & 20 & 80 & II & 4 & 2717.661 & 3 & 10 & II & & 2678.847 & 1 & 3 & II & \\
\hline 2750.477 & $600 \mathrm{R}$ & 500 & II & 4 & 2717.617 & 1 & 4 & II & & 2677.394 & 1 & 500 & III & 3 \\
\hline 2749.907 & 3 & 500 & III & 7 & 2717.106 & 4 & 20 & II & 7 & 2676.131 & 3 & 6 & II & \\
\hline 2749.624 & 3 & 20 & II & & 2716.463 & & 2 & III & & 2674.866 & 3 & 10 & II & 4 \\
\hline 2748.664 & $50 \mathrm{r}$ & 150 & II & 5 & 2716.354 & 3 & 5 & II & & 2673.331 & & 30 & III & \\
\hline 2748.56 & 3 & 10 & II & & 2716.177 & & 3 & II & & 2672.656 & $200 \mathrm{R}$ & 200 & II & 4 \\
\hline 2748.036 & 5 & 30 & II & 4 & 2715.94 & 3 & 15 & II & 7 & 2672.028 & & 2 & II & \\
\hline 2747.641 & 3 & 10 & II & & 2715.640 & 1 & 4 & II & & 2671.958 & $1000 \mathrm{~A}$ & 10 & I & 7 \\
\hline 2747.580 & 20 & 80 & II & 5 & 2715.383 & 4 & 7 & II & & 2671.554 & & 2 & II & \\
\hline 2746.83 & & 1 & II & & 2715.17 & & 1 & II & & 2670.605 & & 1 & II & \\
\hline 2745.712 & 5 & 30 & II & 5 & 2714.408 & 4 & 30 & II & 6 & 2669.305 & & 1 & II & \\
\hline 2745.115 & 2 & 8 & II & & 2714.18 & & 2 & II & & 2669.131 & 1 & 3 & II & \\
\hline 2744.782 & 1 & 3 & II & & 2714.052 & & 1 & III & & 2668.94 & & 2 & II & \\
\hline 2744.372 & & 1 & II & & 2713.718 & & 2 & II & & 2668.752 & 8 & 40 & II & \\
\hline 2742.741 & & 2 & II & & 2712.658 & 15 & 80 & II & 4 & 2668.707 & 4 & 20 & II & 7 \\
\hline 2742.651 & 1 & 5 & II & & 2712.321 & 2 & 400 & III & 3 & 2668.587 & 1 & 3 & II & \\
\hline 2741.779 & 4 & 15 & II & & 2711.785 & 20 & 30 & II & 5 & 2668.092 & & 2 & III & \\
\hline 2741.714 & 8 & 40 & II & 5 & 2711.09 & & 1 & II & & 2667.965 & & 2 & II & \\
\hline 2741.150 & 2 & 8 & II & & 2710.674 & 6 & 30 & II & 6 & 2666.994 & 20 & 2000 & III & 7 \\
\hline 2740.79 & 3 & 9 & II & & 2710.544 & 20 & 80 & II & 5 & 2666.132 & 20 & 2000 & III & 7 \\
\hline 2740.551 & 3 & 6 & II & & 2710.046 & 2 & 5 & II & & 2665.038 & 60 & 100 & II & 6 \\
\hline 2740.397 & 1 & 4 & II & & 2709.715 & 4 & 20 & II & 4 & 2664.894 & & 70 & III & \\
\hline 2740.215 & 2 & 6 & II & & 2708.842 & 5 & 30 & II & 6 & 2664.39 & & 2 & II & \\
\hline 2739.475 & 1 & 2 & II & & 2708.46 & & 2 & II & & 2663.900 & 2 & 5 & II & \\
\hline 2738.818 & 2 & 8 & II & 7 & 2708.035 & & 30 & III & & 2661.888 & & 3 & II & \\
\hline 2738.31 & & 1 & II & & 2705.108 & & 3 & II & & 2660.861 & 1 & 4 & II & \\
\hline 2737.61 & 3 & 15 & II & 5 & 2704.75 & & 2 & II & & 2660.73 & & 2 & II & \\
\hline 2736.553 & 4 & 20 & II & & 2704.53 & 3 & 15 & II & & 2659.983 & & 100 & III & \\
\hline 2736.050 & & 2 & II & & 2702.133 & 1 & 4 & II & & 2659.635 & & 2 & II & \\
\hline 2735.673 & 2 & 6 & II & & 2701.733 & 1 & 4 & II & & 2659.267 & 4 & 20 & II & 4 \\
\hline 2734.928 & & 2 & II & & 2701.130 & 3 & 5 & II & & 2657.46 & & 1 & II & \\
\hline 2734.413 & & 1 & II & & 2700.797 & 6 & 40 & II & 5 & 2657.20 & & 2 & II & \\
\hline 2734.087 & 5 & 30 & II & 7 & 2698.617 & 2 & 8 & II & & 2656.838 & 2 & 15 & II & 5 \\
\hline 2733.724 & & 4 & II & & 2698.447 & & 1 & II & & 2656.125 & 10 & 40 & II & 4 \\
\hline 2733.65 & & 2 & II & & 2696.625 & 4 & 20 & II & 7 & 2653.75 & $1000 \mathrm{R}$ & 1000 & II & 7 \\
\hline 2733.112 & 5 & 10 & II & 5 & 2696.150 & & 2 & II & & 2652.28 & 2 & & I & \\
\hline 2732.942 & 1 & 4 & II & & 2695.556 & & 2 & II & & 2652.25 & 2 & 700 & III & 2 \\
\hline 2732.742 & $30 \mathrm{r}$ & 150 & II & 4 & 2695.427 & 8 & 60 & II & 6 & 2651.74 & 3 & 1000 & III & 2 \\
\hline 2732.50 & & 1 & II & & 2694.99 & & 1 & III & & 2651.68 & 2 & & I & \\
\hline 2732.033 & & 1 & II & & 2694.622 & 1 & 4 & II & & 2650.795 & 2 & 10 & II & \\
\hline 2731.83 & & 1 & II & & 2692.703 & 2 & 10 & II & & 2650.728 & 9 & 30 & II & 4 \\
\hline 2731.573 & & 3 & II & & 2692.408 & 3 & 15 & II & 6 & 2649.787 & 10 & 40 & II & 6 \\
\hline 2731.517 & & 3 & II & & 2691.996 & 5 & 30 & II & 6 & 2649.165 & & 3 & II & \\
\hline 2730.62 & & 1 & II & & 2691.49 & & 3 & II & & 2648.803 & 6 & 30 & II & 4 \\
\hline 2729.50 & 2 & 8 & II & 5 & 2691.44 & & 2 & II & & 2647.455 & 8 & 40 & II & 5 \\
\hline 2729.093 & 1 & 5 & II & & 2691.012 & 2 & 500 & III & 3 & 2647.233 & 2 & 10 & II & \\
\hline 2728.47 & & 1 & II & & 2690.669 & 2 & 2 & II & & 2646.442 & 6 & 30 & II & 5 \\
\hline 2728.420 & 6 & 20 & II & 4 & 2690.298 & 1 & 3 & II & & 2645.691 & & 2 & II & \\
\hline 2727.66 & & 1 & II & & 2689.85 & & 1 & III & & 2644.306 & 20 & 80 & II & 6 \\
\hline 2727.22 & & 1 & II & & 2689.338 & & 2 & III & & 2643.62 & & 100 & III & \\
\hline 2724.65 & 1 & 3 & II & & 2688.22 & & 1 & II & & 2643.237 & & 3 & II & \\
\hline 2724.41 & & 2 & II & & 2687.980 & 4 & 30 & II & 6 & 2642.815 & & 5 & II & \\
\hline 2723.338 & & 3 & II & & 2687.900 & 1 & 5 & II & & 2642.558 & 10 & 1000 & II & 6 \\
\hline 2722.759 & & 2 & II & & 2687.08 & & 1 & II & & 2641.97 & 2 & 10 & II & \\
\hline 2722.475 & 1 & 5 & II & & 2685.990 & 2 & 10 & II & & 2641.886 & 50 & 50 & II & 5 \\
\hline 2722.205 & 8 & 40 & II & 7 & 2685.30 & & 2 & III & & 2641.485 & & 2 & II & \\
\hline 2721.895 & & 3 & II & & 2685.022 & & 2 & III & & 2640.482 & & 300 & III & \\
\hline 2721.183 & & 3 & II & & 2684.747 & 10 & 70 & II & 6 & 2639.448 & 10 & 40 & II & 4 \\
\hline 2720.522 & 1 & 5 & II & & 2683.895 & & 2 & II & & 2638.596 & 4 & 5 & II & \\
\hline 2719.996 & & 2 & II & & 2683.416 & 4 & 30 & II & 6 & 2638.055 & 1 & 500 & III & 3 \\
\hline
\end{tabular}


TABLE 1.-Emission spectra of ytterbium-Continued

\begin{tabular}{|c|c|c|c|c|c|c|c|c|c|c|c|c|c|c|}
\hline \multirow{3}{*}{$\begin{array}{c}\begin{array}{c}\text { Wave- } \\
\text { length } \\
\text { in air }\end{array} \\
\circ \\
\text { A }\end{array}$} & \multicolumn{2}{|c|}{ Intensity } & \multirow{3}{*}{ Spectrum } & \multirow{3}{*}{$\begin{array}{c}\text { Zeeman } \\
\text { type }\end{array}$} & \multirow{3}{*}{$\begin{array}{l}\text { Wave- } \\
\text { length } \\
\text { in air } \\
\\
\AA\end{array}$} & \multicolumn{2}{|c|}{ Intensity } & \multirow{3}{*}{ Spectrum } & \multirow{3}{*}{$\begin{array}{c}\text { Zeeman } \\
\text { type }\end{array}$} & \multirow{3}{*}{$\begin{array}{c}\text { Wave- } \\
\text { length } \\
\text { in air }\end{array}$} & Inte & nsity & & \\
\hline & Meggers & Thompson & & & & Meggers & Thompson & & & & Meggers & Thompson & 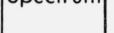 & type \\
\hline & lamp & lamp & & & & lamp & lamp & & & & lamp & lamp & & \\
\hline 2637.637 & & 2 & II & & 2592.694 & & 20 & III & & 2542.318 & 4 & 10 & II & \\
\hline 2637.378 & 2 & 2 & II & & 2591.026 & 3 & 10 & II & 6 & 2541.82 & & 1 & II & \\
\hline 2636.519 & 1 & 6 & II & 6 & 2590.711 & 2 & 5 & II & & 2541.16 & 2 & 6 & II & \\
\hline 2636.399 & & 3 & II & & 2590.328 & & 2 & III & & 2540.60 & & 1 & II & \\
\hline 2636.275 & 2 & 8 & II & & 2588.617 & & 100 & III & & 2539.64 & & 4 & II & \\
\hline 2635.37 & & 30 & III & & 2586.30 & 3 & 10 & II & 7 & 2538.79 & 2 & 20 & II & \\
\hline 2635.197 & 1 & 4 & II & & 2585.67 & & 2 & III & & 2538.67 & $300 \mathrm{~A}$ & 200 & II & 6 \\
\hline 2634.50 & & 2 & III & & 2584.76 & & 2 & III & & 2538.203 & 4 & 30 & II & \\
\hline 2634.306 & 30 & 40 & II & 6 & 2583.748 & 1 & 4 & II & & 2537.65 & 10 & 70 & II & 7 \\
\hline 2634.183 & & 3 & II & & 2582.489 & & 2 & II & & 2537.240 & 1 & 5 & II & \\
\hline 2632.69 & 2 & 8 & II & & 2582.38 & & 7 & III & & 2537.08 & & 1 & II & \\
\hline 2631.73 & 3 & 15 & II & 4 & 2582.068 & & 3 & II & & 2536.962 & 2 & 10 & II & 6 \\
\hline 2630.36 & & 1 & III & & 2581.41 & 1 & 4 & II & & 2536.027 & 4 & 20 & II & 5 \\
\hline 2628.80 & 2 & 7 & II & & 2581.092 & & 3 & II & & 2534.05 & & 2 & II & \\
\hline 2628.605 & 1 & 4 & II & & 2580.36 & 3 & & I & & 2532.55 & 2 & 6 & II & \\
\hline 2628.424 & & 2 & II & & 2579.57 & 4 & 1000 & III & 1 & 2532.19 & 2 & 3 & II & \\
\hline 2628.07 & 3 & 20 & II & 6 & 2577.74 & 3 & 10 & II & & 2531.00 & & 1 & II & \\
\hline 2627.07 & & 300 & III & & 2577.605 & 5 & 20 & II & & 2529.98 & 1 & 3 & II & \\
\hline 2624.949 & 3 & 5 & II & & 2576.09 & & 2 & II & & 2529.50 & 3 & 9 & II & \\
\hline 2624.52 & & 2 & II & & 2574.78 & 2 & 10 & II & & 2529.14 & & 20 & III & \\
\hline 2623.22 & 2 & 9 & II & 4 & 2573.147 & 6 & 30 & II & 7 & 2528.836 & 1 & 1 & II & \\
\hline 2621.67 & 4 & 20 & II & & 2572.15 & 1 & 4 & II & & 2528.339 & 3 & 25 & II & 7 \\
\hline 2621.11 & 1 & 600 & III & 3 & 2571.69 & 1 & 2 & II & & 2527.954 & & 3 & II & \\
\hline 2619.93 & 3 & 15 & II & & 2571.36 & 7 & 50 & II & 4 & 2527.864 & 4 & 30 & II & 5 \\
\hline 2619.074 & 4 & 20 & II & 5 & 2569.61 & 1 & 2 & II & & 2527.49 & & 2 & II & \\
\hline 2618.948 & & 2 & II & & 2568.60 & & 2 & II & & 2527.165 & & 2 & III & \\
\hline 2618.800 & 40 & 1 & I & & 2568.17 & 3 & 10 & II & & 2526.31 & 2 & 5 & II & \\
\hline 2617.01 & $100 \mathrm{r}$ & 100 & II & 6 & 2567.607 & 7 & 2000 & III & 7 & 2526.189 & & 3 & II & \\
\hline 2615.35 & 1 & 5 & II & & 2566.78 & & 100 & III & & 2524.990 & 2 & 10 & II & \\
\hline 2615.262 & 6 & 30 & II & 4 & 2565.573 & 10 & 40 & II & & 2524.573 & 4 & 8 & II & \\
\hline 2614.55 & 1 & 3 & II & & 2564.439 & & 5 & III & & 2524.30 & & 3 & III & \\
\hline 2614.45 & 2 & 3 & II & & 2564.32 & & 3 & III & & 2522.438 & 15 & 50 & II & 6 \\
\hline 2612.63 & 2 & 10 & II & & 2563.92 & 2 & 2 & II & & 2522.07 & & 15 & III & \\
\hline 2612.041 & 4 & 20 & II & & 2561.66 & & 10 & III & & 2521.04 & 3 & 15 & II & \\
\hline 2611.645 & 2 & 2 & II & & 2560.56 & & 100 & III & & 2520.329 & & 8 & III & \\
\hline 2610.860 & 5 & 30 & II & 7 & 2559.972 & 2 & 5 & II & & 2519.39 & & 2 & II & \\
\hline 2610.531 & 1 & 4 & II & & 2559.094 & & 3 & III & & 2518.59 & & 2 & II & \\
\hline 2609.14 & & 30 & III & & 2558.40 & 3 & 2 & II & & 2518.47 & & 1 & II & \\
\hline 2608.498 & 1 & 4 & II & & 2557.804 & 4 & 5 & II & & 2518.01 & 3 & 10 & II & 6 \\
\hline 2607.864 & 5 & 30 & II & 4 & 2557.703 & 1 & 5 & и & & 2516.82 & 50 & 300 & II & 7 \\
\hline 2607.22 & & 2 & III & & 2557.259 & 4 & 20 & II & 7 & 2516.35 & 6 & 30 & II & 6 \\
\hline 2605.43 & 2 & 3 & II & & 2556.30 & & 3 & II & & 2516.118 & 1 & & I & \\
\hline 2604.69 & & 2 & II & & 2555.29 & 1 & 300 & III & 3 & 2516.031 & 3 & 15 & II & \\
\hline 2604.58 & & 4 & III & & 2554.641 & & 2 & II & & 2515.600 & 2 & 7 & II & 7 \\
\hline 2604.053 & 5 & 30 & II & 5 & 2552.88 & 2 & 2 & II & & 2515.460 & 2 & 3 & II & \\
\hline 2602.40 & & 2 & II & & 2552.70 & 10 & 60 & II & 7 & 2515.165 & & 2 & II & \\
\hline 2601.87 & 20 & 1 & I & & 2552.147 & 15 & 80 & II & 7 & 2514.69 & & 1 & II & \\
\hline 2600.850 & 2 & 9 & II & & 2550.786 & & 1 & II & & 2514.248 & 7 & 20 & II & 7 \\
\hline 2600.508 & 1 & 2 & II & & 2550.68 & 3 & 10 & II & & 2513.64 & & 1 & II & \\
\hline 2600.21 & 3 & 15 & II & & 2550.39 & & 40 & III & & 2512.87 & & 2 & II & \\
\hline 2599.75 & 1 & 2 & II & & 2550.060 & 10 & 20 & II & & 2512.524 & 5 & 20 & II & 5 \\
\hline 2599.144 & 1 & 800 & III & 1 & 2550.023 & & 3 & II & & 2512.061 & $50 \mathrm{r}$ & 100 & II & 4 \\
\hline 2598.43 & 2 & 6 & II & & 2548.756 & 3 & 15 & II & 7 & 2511.868 & 4 & 20 & II & \\
\hline 2598.11 & & 2 & II & & 2548.00 & & 3 & II & & 2510.50 & 2 & 7 & II & \\
\hline 2597.69 & 1 & 3 & II & & 2547.498 & 2 & 8 & II & & 2510.29 & 1 & 2 & II & \\
\hline 2597.619 & 2 & 2 & II & & 2547.23 & 2 & 5 & II & & 2510.18 & & 1 & III & \\
\hline 2597.495 & 1 & 5 & II & & 2547.20 & & 2 & II & & 2510.03 & 1 & 1 & II & \\
\hline 2597.23 & 1 & 500 & III & 2 & 2546.886 & 1 & 2 & II & & 2509.86 & 2 & 5 & II & \\
\hline 2596.735 & 3 & 30 & II & & 2546.28 & 2 & 5 & II & & 2508.073 & 10 & 20 & II & \\
\hline 2596.320 & 6 & 80 & II & 6 & 2545.871 & 2 & 4 & II & & 2506.69 & & 1 & III & \\
\hline 2596.16 & 6 & 80 & II & 6 & 2545.545 & & 3 & II & & 2506.52 & & 4 & III & \\
\hline 2594.493 & 3 & 20 & II & & 2544.72 & & 2 & II & & 2506.25 & & 40 & III & \\
\hline 2594.20 & 3 & 15 & II & 6 & 2542.76 & 3 & 10 & II & & 2505.477 & 6 & 30 & II & 6 \\
\hline
\end{tabular}


TABLE 1.-Emission spectra of ytterbium-Continued

\begin{tabular}{|c|c|c|c|c|c|c|c|c|c|c|c|c|c|c|}
\hline \multirow{3}{*}{$\begin{array}{c}\text { Wave- } \\
\text { length } \\
\text { in air }\end{array}$} & \multicolumn{2}{|c|}{ Intensity } & \multirow{3}{*}{ Spectrum } & \multirow{3}{*}{$\begin{array}{c}\text { Zeeman } \\
\text { type }\end{array}$} & \multirow{3}{*}{$\begin{array}{c}\text { Wave- } \\
\text { length } \\
\text { in air }\end{array}$} & \multicolumn{2}{|c|}{ Intensity } & \multirow{3}{*}{ Spectrum } & \multirow{3}{*}{$\begin{array}{c}\text { Zeeman } \\
\text { type }\end{array}$} & \multirow{3}{*}{$\begin{array}{c}\begin{array}{c}\text { Wave- } \\
\text { length } \\
\text { in air }\end{array} \\
\AA \\
\end{array}$} & & nsity & & \\
\hline & Meggers & Thompson & & & & Meggers & Thompson & & & & Meggers & Thompson & thecinom & type \\
\hline & lamp & lamp & & & & lamp & lamp & & & & lamp & lamp & & \\
\hline 2503.46 & & 1 & II & & 2449.45 & & 2 & II & & 2398.02 & 40 & 20 & II & \\
\hline 2502.02 & 30 & 70 & II & 4 & 2447.26 & 3 & 10 & II & & 2397.87 & & 3 & II & \\
\hline 2501.21 & 1 & 3 & II & & 2446.41 & & 1 & II & & 2397.73 & & 3 & II & \\
\hline 2500.568 & 2 & 6 & II & & 2445.92 & 1 & 2 & II & & 2397.34 & & 1 & II & \\
\hline 2498.73 & & 2 & II & & 2444.64 & & 2 & II & & 2390.74 & $20 \mathrm{R}$ & 70 & II & 4 \\
\hline 2498.36 & 2 & 5 & II & & 2443.74 & & 2 & II & & 2390.57 & 2 & 8 & II & \\
\hline 2496.85 & & 2 & II & & 2442.64 & 1 & 3 & II & & 2389.18 & & 3 & II & \\
\hline 2495.63 & 5 & 20 & II & & 2440.43 & & 20 & III & & 2388.40 & 2 & 7 & II & \\
\hline 2495.05 & 4 & 3 & II & & 2440.01 & & 1 & II & & 2386.75 & & 2 & II & \\
\hline 2493.64 & 3 & 10 & II & & 2439.72 & & 2 & II & & 2385.94 & & 1 & II & \\
\hline 2493.50 & & 2 & III & & 2439.59 & 3 & 5 & II & & 2385.01 & 1 & 5 & II & \\
\hline 2491.69 & & 20 & III & & 2439.31 & & 20 & III & & 2383.44 & & 1 & II & \\
\hline 2491.15 & 3 & 5 & II & & 2438.27 & & 100 & III & & 2382.98 & & 2 & II & \\
\hline 2490.42 & & 200 & III & 7 & 2437.24 & 4 & 7 & II & & 2382.57 & 1 & 10 & II & \\
\hline 2488.92 & & 3 & II & & 2436.87 & 1 & 2 & II & & 2380.39 & 2 & 15 & II & \\
\hline 2487.08 & 1 & 3 & II & & 2436.06 & & 5 & II & & 2377.22 & & 20 & III & \\
\hline 2484.89 & 5 & 20 & II & 6 & 2434.71 & 1 & 9 & III & & 2375.18 & & 3 & II & \\
\hline 2484.29 & & 5 & III & & 2434.02 & & 2 & II & & 2374.74 & & 1 & II & \\
\hline 2481.42 & 1 & 3 & II & & 2433.74 & & 1 & II & & 2374.52 & 1 & 5 & II & \\
\hline 2481.35 & & 2 & II & & 2433.62 & 1 & 3 & II & & 2374.32 & & 3 & II & \\
\hline 2481.03 & 1 & 2 & II & & 2433.43 & & 20 & III & & 2373.89 & 2 & 20 & II & 7 \\
\hline 2479.29 & & 2 & II & & 2433.16 & 1 & 2 & II & & 2373.06 & & 7 & II & \\
\hline 2479.15 & 1 & 5 & II & & 2433.05 & & 1 & II & & 2371.64 & & 1 & II & \\
\hline 2477.76 & & 1 & II & & 2432.62 & 2 & 3 & II & & 2369.99 & & 30 & III & \\
\hline 2476.62 & & 2 & II & & 2432.01 & & 2 & II & & 2369.59 & & 2 & II & \\
\hline 2475.92 & & 2 & II & & 2431.69 & & 1 & II & & 2369.42 & & 50 & II & \\
\hline 2474.52 & 2 & 1 & II & & 2429.18 & & 10 & III & & 2367.46 & & 50 & III & \\
\hline 24.74 .27 & 1 & 3 & II & & 2429.01 & 1 & 10 & II & & 2366.97 & & 2 & II & \\
\hline 2473.95 & & 1 & II & & 2428.74 & & 1 & II & & 2365.68 & & 2 & III & \\
\hline 2473.16 & & 2 & II & & 2428.30 & & 1 & II & & 2365.43 & & 200 & III & \\
\hline 2471.06 & 2 & 3 & II & & 2428.12 & & 6 & II & & 2364.10 & & 2 & II & \\
\hline 2470.85 & & 2 & II & & 2427.66 & & 1 & II & & 2363.49 & 1 & 9 & II & \\
\hline 2469.89 & 1 & 2 & II & & 2427.20 & & 1 & II & & 2362.89 & 10 & 60 & II & \\
\hline 2469.52 & & 1 & II & & 2426.19 & & 1 & II & & 2362.30 & & 1 & II & \\
\hline 2467.24 & & 2 & II & & 2425.68 & & 1 & II & & 2361.08 & & 40 & III & \\
\hline 2466.63 & 5 & 20 & II & 6 & 2424.61 & 4 & 6 & II & & 2358.67 & & 2 & II & \\
\hline 2465.13 & & 2 & II & & 2423.96 & & 1 & II & & 2358.53 & & 6 & III & \\
\hline 2464.59 & 1 & 10 & II & & 2422.84 & 10 & 20 & II & & 2357.84 & 1 & 7 & II & \\
\hline 2464.50 & $1000 \mathrm{~A}$ & 10 & I & 7 & 2421.35 & 10 & 50 & II & 4 & 2355.03 & & 1 & II & \\
\hline 2463.79 & & 1 & II & & 2420.03 & 1 & 4 & II & & 2350.06 & & 2 & II & \\
\hline 2463.35 & 2 & 4 & II & & 2419.41 & & 1 & II & & 2349.39 & & 7 & II & \\
\hline 2463.04 & & 1 & II & & 2419.20 & & 2 & II & & 2347.41 & & 1 & II & \\
\hline 2462.71 & & 1 & II & & 2418.38 & & 2 & II & & 2345.63 & & 3 & III & \\
\hline 2461.98 & & 2 & II & & 2417.01 & & 3 & II & & 2344.66 & 4 & 20 & II & \\
\hline 2461.83 & & 1 & II & & 2415.39 & & 2 & II & & 2344.09 & 4 & 7 & II & \\
\hline 2461.40 & 3 & 10 & II & & 2414.33 & 2 & 6 & II & & 2340.78 & & 1 & II & \\
\hline 2460.25 & 10 & 40 & II & 7 & 2413.77 & & 2 & II & & 2340.40 & 1 & 6 & II & \\
\hline 2460.00 & 1 & 1 & II & & 2413.66 & 1 & 5 & II & & 2339.61 & & 1 & II & \\
\hline 2459.93 & 1 & 4 & II & & 2412.33 & & 60 & III & & 2337.97 & & 200 & III & \\
\hline 2458.637 & & 10 & III & & 2411.53 & & 9 & III & & 2336.83 & & 1 & II & \\
\hline 2458.22 & & 2 & II & & 2410.77 & & 8 & III & & 2335.44 & 2 & 10 & II & \\
\hline 2457.73 & & 2 & II & & 2410.04 & & 20 & III & & 2335.11 & & 1 & II & \\
\hline 2456.74 & & 1 & II & & 2409.44 & & 1 & II & & 2333.28 & & 7 & III & \\
\hline 2456.35 & & 1 & II & & 2409.06 & & 5 & II & & 2332.28 & & 1 & II & \\
\hline 2456.08 & & 6 & II & & 2407.98 & & 1 & II & & 2331.56 & & 1 & II & \\
\hline 2455.97 & & 3 & II & & 2407.76 & & 1 & II & & 2330.39 & & 1 & II & \\
\hline 2454.75 & 1 & 10 & II & & 2406.79 & & 1 & II & & 2328.06 & & 1 & II & \\
\hline 2454.52 & 3 & 20 & II & & 2406.09 & 1 & 10 & II & & 2327.74 & & 3 & II & \\
\hline 2453.51 & & 2 & II & & 2404.62 & & 2 & III & & 2327.38 & & 1 & II & \\
\hline 2451.94 & 2 & 3 & II & & 2403.95 & & 50 & III & & 2326.92 & 1 & 10 & II & \\
\hline 2451.15 & & 1 & II & & 2403.41 & & 4 & II & & 2324.44 & 30 & 9 & I & \\
\hline 2450.26 & 3 & 5 & II & & 2402.71 & & 2 & II & & 2323.18 & & 4 & II & \\
\hline 2449.71 & 1 & 3 & II & & 2399.93 & & 1 & II & & 2323.02 & & 1 & II & \\
\hline
\end{tabular}


TABLE 1.-Emission spectra of ytterbium-Continued

\begin{tabular}{|c|c|c|c|c|c|c|c|c|c|c|c|c|c|c|}
\hline \multirow{3}{*}{$\begin{array}{l}\text { Wave- } \\
\text { length } \\
\text { in air }\end{array}$} & \multicolumn{2}{|c|}{ Intensity } & \multirow{3}{*}{ Spectrum } & \multirow{3}{*}{$\begin{array}{c}\text { Zeeman } \\
\text { type }\end{array}$} & \multirow{3}{*}{$\begin{array}{l}\text { Wave- } \\
\text { length } \\
\text { in air } \\
\circ \\
\text { A }\end{array}$} & \multicolumn{2}{|c|}{ Intensity } & \multirow{3}{*}{ Spectrum } & \multirow{3}{*}{$\begin{array}{c}\text { Zeeman } \\
\text { type }\end{array}$} & \multirow{3}{*}{$\begin{array}{l}\text { Wave- } \\
\text { length } \\
\text { in air } \\
\AA \\
\end{array}$} & \multicolumn{2}{|c|}{ Intensity } & \multirow{3}{*}{ Spectrum } & \multirow{3}{*}{$\begin{array}{c}\text { Zeeman } \\
\text { type }\end{array}$} \\
\hline & \multirow{2}{*}{$\begin{array}{c}\text { Meggers } \\
\text { lamp }\end{array}$} & \multirow{2}{*}{$\begin{array}{c}\text { Thompson } \\
\text { lamp }\end{array}$} & & & & \multirow{2}{*}{$\begin{array}{l}\text { Meggers } \\
\text { lamp }\end{array}$} & \multirow{2}{*}{\begin{tabular}{|c} 
Thompson \\
lamp
\end{tabular}} & & & & \multirow{2}{*}{$\begin{array}{l}\text { Meggers } \\
\text { lamp }\end{array}$} & \multirow{2}{*}{$\begin{array}{c}\text { Thompson } \\
\text { lamp }\end{array}$} & & \\
\hline & & & & & & & & & & & & & & \\
\hline 2322.63 & & 1 & II & & 2263.12 & & 3 & II & & 2148.60 & & 5 & III & \\
\hline 2321.38 & & 1 & II & & 2262.26 & & 100 & III & & 2144.77 & & $15 \mathrm{e}$ & IV & \\
\hline 2320.81 & $200 \mathrm{~A}$ & 150 & II & 4 & 2257.03 & & 200 & III & & 2142.21 & & 2 & II & \\
\hline 2318.65 & & 2 & II & & 2255.48 & & 3 & II & & 2139.98 & & $10 \mathrm{e}$ & IV & \\
\hline 2315.20 & 3 & 10 & II & & 2253.97 & & 1 & II & & 2138.35 & & $4 \mathrm{e}$ & IV & \\
\hline 2314.81 & & 8 & III & & 2253.27 & & 2 & II & & 2137.71 & & 4 & II & \\
\hline 2314.49 & 1 & 200 & III & 7 & 2251.93 & & 1 & II & & 2135.27 & & 3 & II & \\
\hline 2312.59 & 1 & 3 & II & & 2244.28 & & 100 & III & & 2131.40 & 6 & 5 & II & \\
\hline 2311.18 & & 1 & II & & 2243.18 & & 1 & II & & 2126.74 & 100 & 80 & II & \\
\hline 2309.54 & 2 & 5 & II & & 2240.11 & & 300 & III & & 2125.57 & & 4 & II & \\
\hline 2309.27 & & 100 & III & & 2237.51 & & 2 & II & & 2120.36 & & 3 & II & \\
\hline 2307.39 & 1 & 6 & II & & 2231.56 & & 5 & III & & 2119.18 & & 20 & III & \\
\hline 2306.30 & & 2 & II & & 2227.71 & & 5 & III & & 2117.62 & & 6 & II & \\
\hline 2305.32 & 2 & 300 & III & 1 & 2224.89 & & 1 & II & & 2116.67 & 100 & 80 & II & \\
\hline 2303.28 & & 2 & II & & 2224.46 & 100 & 80 & II & 4 & 2114.56 & & 3 & III & \\
\hline 2303.16 & & 1 & II & & 2222.25 & & 1 & II & & 2113.39 & & 1 & III & \\
\hline 2298.67 & & 2 & II & & 2216.20 & & 1 & II & & 2110.20 & & 4 & III & \\
\hline 2297.91 & & 4 & II & & 2214.68 & & 3 & II & & 2109.54 & & 50 & III & \\
\hline 2296.63 & & 2 & II & & 2213.06 & & 1 & II & & 2106.71 & & 10 & III & \\
\hline 2292.83 & & 4 & II & & 2212.60 & 3 & & I & & 2102.73 & 5 & 20 & II & \\
\hline 2292.19 & & 3 & II & & 2211.91 & & 1 & II & & 2102.10 & & 4 & III & \\
\hline 2291.61 & & 1 & II & & 2203.11 & & 2 & II & & 2098.36 & & 30 & III & \\
\hline 2289.76 & 4 & & I & & 2202.27 & & 80 & III & & 2096.79 & & 15 & III & \\
\hline 2288.96 & 2 & 10 & II & & 2201.20 & & 3 & II & & 2095.31 & & 80 & III & \\
\hline 2288.14 & & 1 & II & & 2198.14 & & 20 & III & & 2094.77 & & 10 & III & \\
\hline 2288.04 & 1 & & I & & 2189.45 & & 6 & II & & 2093.13 & & 6 & III & \\
\hline 2285.79 & & 4 & II & & 2185.71 & 100 & 80 & II & 5 & 2092.26 & & 20 & III & \\
\hline 2284.99 & & 30 & II & & 2178.70 & & 4 & II & & 2091.23 & & 20 & III & \\
\hline 2283.40 & 3 & 9 & II & & 2174.28 & 1 & 3 & II & & 2087.98 & & 50 & III & \\
\hline 2282.99 & 1 & 150 & III & & 2169.79 & & 2 & II & & 2087.37 & & 10 & III & \\
\hline 2279.44 & & 1 & II & & 2165.21 & & 3 & II & & 2086.53 & & 4 & III & \\
\hline 2276.11 & & 1 & II & & 2163.89 & & $4 \mathrm{e}$ & IV & & 2078.05 & & 30 & III & \\
\hline 2275.07 & & 3 & II & & 2161.60 & 10 & 40 & II & & 2073.64 & & 10 & III & \\
\hline 2271.31 & & 1 & II & & 2160.20 & & 6 & II & & 2067.52 & & 4 & III & \\
\hline 2271.11 & 40 & 2 & I & & 2159.89 & & 1 & II & & 2066.49 & & 10 & III & \\
\hline 2269.57 & & 1 & II & & 2155.88 & 3 & & I & & 2054.80 & & 20 & III & \\
\hline 2268.57 & & 4 & II & & 2155.50 & & 30 & II & & 2029.54 & & 6 & III & \\
\hline 2268.31 & & 3 & II & & 2155.20 & & 3 & II & & 2022.03 & & 2 & II & \\
\hline 2267.16 & & 3 & II & & 2154.19 & & $20 \mathrm{e}$ & IV & & 2021.36 & & 2 & II & \\
\hline 2265.67 & & 200 & III & & 2152.32 & & 3 & II & & 2018.08 & & 2 & II & \\
\hline 2263.89 & & 1 & II & & 2148.94 & & 2 & II & & 1998.17 & & 40 & III & \\
\hline
\end{tabular}


The senior author gratefully acknowledges the cooperation of George R. Harrison in obtaining the first Zeeman spectrograms of ytterbium in 1939. He is happy to thank Jack Sugar for the first Yb spectrograms showing reversals that he made in 1961, and Nissan Spector for infrared spectrograms of the sliding spark in 1963. He also thanks Mrs. John B. Peterson most sincerely for her expert assistance with wavelength calculations and compilations of many thousands of ytterbium lines.

\section{References}

[1] W. F. Meggers and B. F. Scribner, Arc and spark spectra of ytterbium, J. Res. NBS 19, 651-664 (1937), RP1053.

[2] W. F. Meggers, The second spectrum of ytterbilim, J. Opt. Soc. Am. 37, 988 A (1947).

[3] A. Gatterer and J. Junkes, Spektren der Seltenen Erden, pp. 307-314 (Vatican City Press, 1945).

[4] C. J. Humphreys and E. Paul, Jr., Infrared Atomic Spectra, Quarterly Report Foundational Research Projects NOVORD Report 5970, NOLC Report 473, 57-67 (1959).

[5] B. W. Bryant, The spectra of doubly and triply ionized ytterbium, Johns Hopkins Spectroscopic Report Number 21, 82 pp. (1961).

[6] L. Allen, The Zeeman effect of the arc and spark spectra of ytterbium and thulium, United Kingdom Atomic Energy Authority Research Group Report, AERE-R4029, 34 pp (1962).
[7] W. F. Meggers, Present experimental status of rare-earth spectra, J. Opt. Soc. Am. 50, 405-406 (1960).

[8] C. H. Corliss, W. R. Bozman, and F. O. Westfall, Electrodeless metal-halide lamps, J. Opt. Soc. Am. 43, 398-400 (1953).

[9] C. H. Corliss and W. F. Meggers, Improved description of hafnium spectra, J. Res. NBS 61, 269-324 (1958), RP2904.

[10] W. F. Meggers and F. O. Westfall, Lamps and wavelengths of mercury 198, J. Res. NBS 44, 447-455 (1950), RP2091.

[11] M. Zelikoff, P. H. Wyckoff, L. M. Aschenbrand, and R. S. Loomis, Electrodeless discharge lamps containing metal vapors, J. Opt. Soc. Am. 42, 818-819 (1952).

[12] J. J. Thompson, On the discharge of electricity through exhausted tubes without electrodes, Phil. Mag. 32, 321-336, 445-464 (1891).

[13] V. L. Bloch and E. Bloch, Spectres d'Etincelle d'Ordre Superieur du Mercure, J. Phys. Rad. 4, 333-348 (1923).

[14] J. Sugar, Light source for producing self-reversed spectral lines, J. Res. NBS 66A (Phys. and Chem.), No. 4, 321-324 (1962).

[15] J. Sugar, Analysis of the third spectrum of praseodymium, J. Opt. Soc. Am. 53, 831-839 (1963).

[16] G. R. Harrison and F. Bitter, Zeeman effects in complex spectra at fields up to 100,000 gauss, Phys. Rev. 57, 15-20 (1940).

[17] W. F. Meggers and C. H. Corliss, First spectrum of ytterbium, Yb I, J. Opt. Soc. Am. 50, 1136A (1960).

[18] E. Back and A. Landé, Zeemaneffekt und Multiplett-struktur der Spektrallinien, p. 168 (Julius Springer, Berlin, 1925).

Washington, August 10, 1965.
(Paper 70Al-386) 